\title{
Low Dimensional Morphology Analysis and Computational Optimization of Flapping Propulsors in Nature
}

\author{
A Dissertation \\ Presented to \\ the faculty of the School of Engineering and Applied Science \\ University of Virginia
}

In Partial Fulfillment of the

Requirements for the Degree

of

Doctor of Philosophy

By

Yan Ren

July 2016 


\section{APPROVAL SHEET}

The dissertation is submitted in partial fulfillment of the

requirements for the degree of

Doctor of Philosophy

Yan Ren, Author

This dissertation has been read and approved by the examining Committee:

Haibo Dong, Advisor

Gavin T. Garner, Committee Chair

Eric Loth, Committee Member

Matthew A. Reidenbach, Committee Member

Baoxing $\mathrm{Xu}$, Committee Member

Accepted for the School of Engineering and Applied Science:

Craig H. Benson, Dean

School of Engineering and Applied Science

July 2016 


\begin{abstract}
Flapping propulsion is widely adopted by many natural flyers/swimmers, including insects, birds, fishes, and marine mammals. It offers an attractive alternative to conventional propulsion methods for future bio-inspired aerial/underwater systems. However, due to lack of effective technology of studying the highly complex propulsor morphing kinematics and its associated aero/hydrodynamics, achieving biological levels of aero/hydro-performance in bio-inspired flapping propulsor design has proven elusive. Here, an integrated experimental and computational methodology has been developed to systematically study the flapping propulsion system in nature. The goal is to advance the fundamental knowledge of biological fluid dynamics in animal flight/swimming, and provide guidance for future optimal designs of bio-inspired flapping propulsors.
\end{abstract}

The current dissertation consists of two parts, tools development and analysis of propulsion systems. In the first part, the integrated methodology is introduced, and the corresponding major contributions of the work are: (1) a highly versatile and accurate jointbased surface reconstruction method is developed to quantify the propulsor flexion and body kinematics of animals in free flight/swimming; (2) a spherical-coordinates-based singular value decomposition (SSVD) method is developed to perform low dimensional morphology analysis of flapping propulsors in nature; (3) an immersed boundary method for deformable attaching bodies (IBM-DAB) is developed to handle direct numerical simulations (DNS) in some extreme situations which are commonly exist in nature, including solid body with sharp edge and with deformable attaching membrane bodies; (4) 
a highly efficient gradient-based parallel curve searching optimizer is developed to explore design space of flapping propulsors.

In the second part, the aforementioned integrated approach is applied to study several problems. We first investigate the optimal configurations of several morphological parameters, which control the dynamic camber and twisting of the propulsors, on aerodynamic performance using simplified canonical models. Optimizations of dynamic camber formation of 2D pitching-plunging plates and dynamic twisting of 3D pitchingrolling plates are performed. It is found that the morphological parameters play important roles in the plate aerodynamic performance and wake structures. Comparing to completely rigid plate, the thrust production and propulsive efficiency of optimized plates can be improved up to $29.1 \%$ and $43.1 \%$, respectively. The associated flow mechanisms are found to be the improved strength and attachment of leading-edge vortex (LEV).

Next, the integrated approach is used to study the complex morphing propulsor kinematics and the associated aero/hydrodynamics of natural flyers/swimmers in relatively simple motions, such as hovering and fast swimming. The SSVD analysis of the forewing motion of a hovering dragonfly reveals that the complicated wing motion can be represented by a low dimensional model contains two dominant SSVD modes, a flapping mode and a morphing mode. The low dimensional model contains $92 \%$ of the original motion, and can recover up to $96 \%$ of the aerodynamic performance. Similar analysis is performed on the morphing fluke kinematics of a fast swimming orca. The results show that two dominant modes, a spanwise morphing mode and a chordwise morphing mode can be identified. The low dimensional model consists of these two modes contains $74.3 \%$ of the original motions, and can fully recover the hydrodynamic performance. In addition, 
a unique tri-ring vortex structure, which is closely related to the biology of cetaceans, is found in the wake of the swimming orca. Parametric studies on the aero/hydrodynamic role of those dominant modes reveal that the morphing modes (including the morphing mode of the dragonfly wing and the spanwise and chordwise morphing modes of the orca fluke) amplitudes and phases are critical control parameters to achieve optimal aero/hydrodynamic performance. We further investigate optimal configurations of dominant modes on aerodynamic performance for the dragonfly wing. The corresponding optimized low dimensional wing models, which can beyond biological levels of aerodynamic performance, are obtained. The associated flow mechanisms are found to be the improved LEV attachment and the reduced TV strength.

In the last part of the dissertation, the integrated approach is extended to study the most complex propulsion system in nature. The 3D wake structures and aerodynamic performance of a freely maneuvering hummingbird is studied in detail. Our simulation results show asymmetric wake structure between inner and outer wings of the hummingbird. A unique duel-ring vortex structure, which is the source of the wake asymmetry, is found in the wake of one of the two wings of the hummingbird. The duelring vortex structure corresponds to larger wing twisting and lower drag production, which creates unbalanced aerodynamic forces to help with the maneuver.

In the future, the extension of this work will be on the SSVD analysis and computational optimization of highly complex flapping propulsion systems, such as maneuvering birds/insects, burst-and-coast fishes, etc. The methodology and findings of this work have the potential to bring new insights into the future design of highperformance bio-inspired systems. 


\section{Acknowledgements}

I would like to express my heartfelt gratitude and sincere thanks to my research advisor Dr. Haibo Dong, Associate Professor of Mechanical Engineering, Department of Mechanical and Aerospace Engineering at University of Virginia. Without his guidance, constant supervision, and valuable suggestions throughout the entire period of the work, it would have been impossible to complete the research work. I am thankful to my parents for all their support throughout my life. I am also greatly thankful to Dr. Geng Liu, Dr. Chengyu Li, Dr. Samane Zeyghami, Mr. Pan Han, and all other Flow Simulation Research Group (FSRG) members for their assistance, generosity and advice throughout this study.

I am very grateful to the faculty and staff of the Department of Mechanical and Aerospace Engineering at University of Virginia for providing me with the opportunity to pursue higher studies.

This work was supported by AFOSR FA9550-12-1-0071, AFOSR FA9550-11-1-0058, ONR MURI N00014-08-1-0642, and National Science Foundation CBET-1313217. 


\section{Table of Contents}

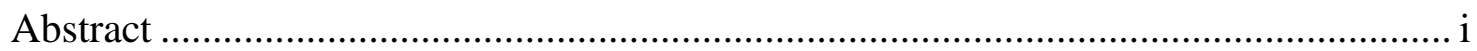

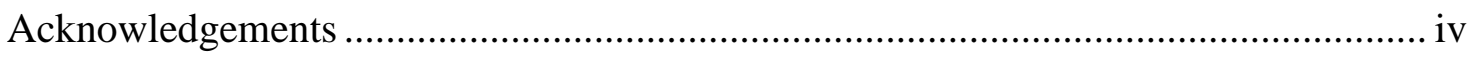

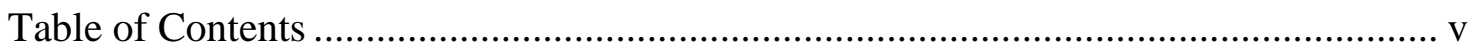

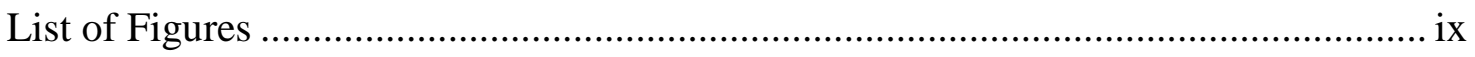

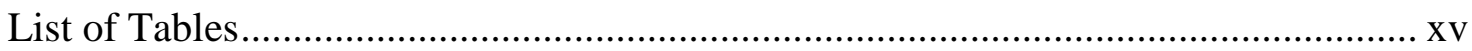

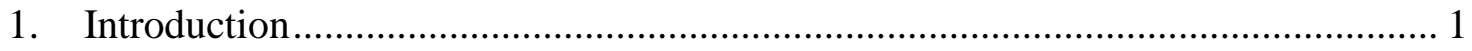

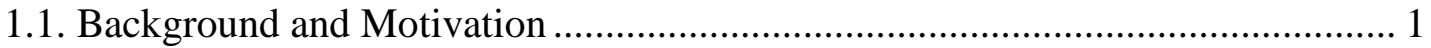

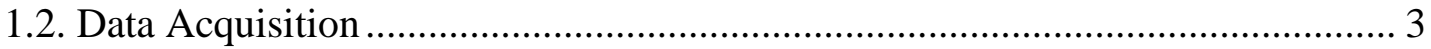

1.3. Effect of Propulsor Morphing Kinematics ............................................... 4

1.4. Singular Value Decomposition (SVD) ................................................. 6

1.5. Optimum Design of Flapping Propulsors ................................................... 7

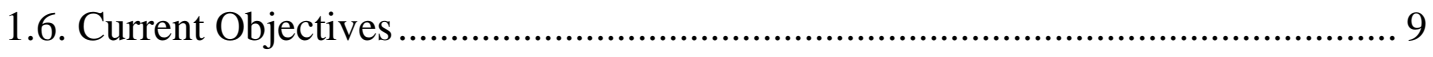

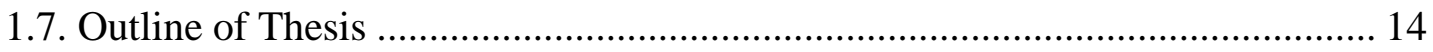

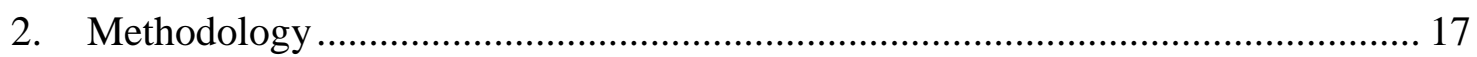

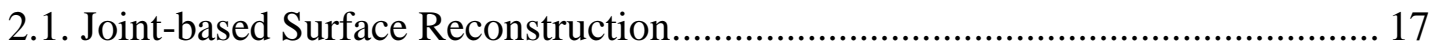

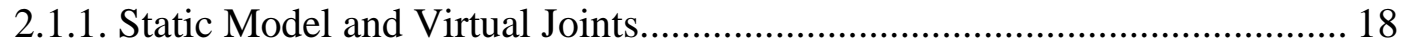

2.1.2. Virtual Cameras and Reconstruction .................................................. 19

2.1.3. Validation: Deformable Wing Kinematics of a Hovering Dragonfly.......... 21

2.2. Spherical-coordinates-based Singular Value Decomposition (SSVD) ............. 25

2.2.1. Coordinate Transformation .................................................................... 26

2.2.2. Reference Snapshot Determination................................................... 27

2.2.3. Displacement Matrix and Singular Value Decomposition ........................ 28

2.2.4. Low-dimensional Models .................................................................. 33 
2.2.5. Convergence Studies and Motion Percentage.............................................. 34

2.2.6. SSVD Analysis of Various Flapping Propulsors ........................................... 36

2.3. Immersed Boundary Method for Deformable Attaching Bodies (IBM-DAB) .. 38

2.3.1. Governing Equations and Discretization Scheme......................................... 39

2.3.2. Immersed Boundary and Cell Identification............................................... 42

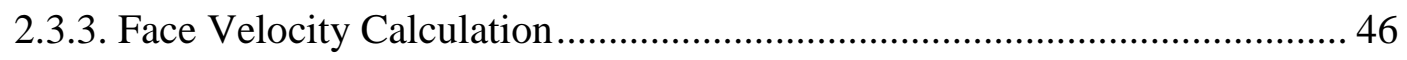

2.3.4. Conflicting Face Velocity Correction and Gap Filling between Solid and

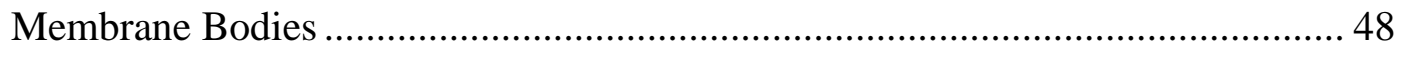

2.3.5. Validation: Steady Swimming of a Baby Trout........................................... 52

2.4. Gradient-based Computational Optimization ...................................................... 56

2.4.1. Sequential Quadratic Programming (SQP) ................................................. 57

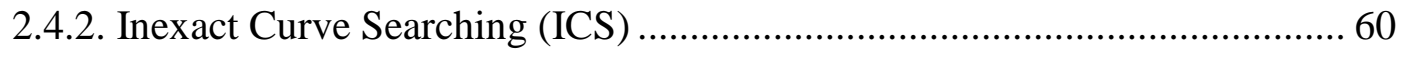

2.4.3. Validation: Optimization of a 2D Hovering Plate with Dynamic Trailing-Edge

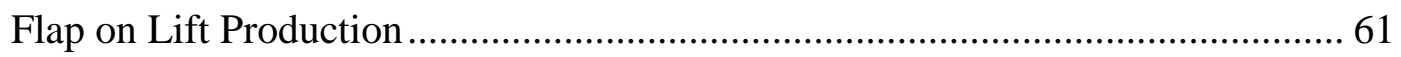

3. Computational Optimization of Flexible Wing Aerodynamic Performance in

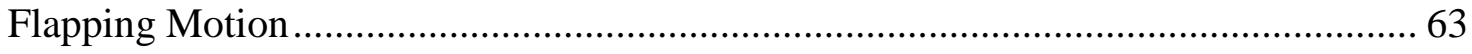

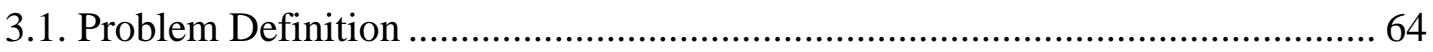

3.2. Optimization of Thrust Production and Propulsive Efficiency ............................ 66

3.3. Wake Structures of the Optimized Wing Models................................................ 69

3.4. Chapter Summaries.......................................................................................... 72

4. Computational Optimization of Dynamic Twisting of Pitching-rolling Plates on Aerodynamic Performance..................................................................................... 74

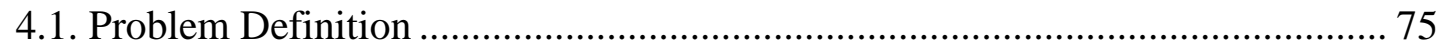

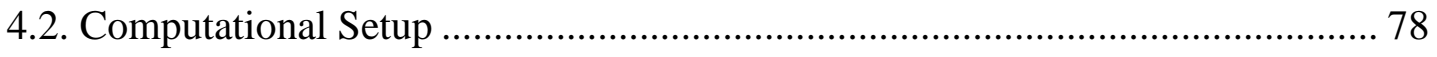

4.3. Pitching-rolling Plate without Surface Morphing …………………………...... 79

4.4. Pitching-rolling Plates with Optimal Surface Morphing ..................................... 82 
4.5. Chapter Summaries.

5. Aerodynamics of Low Dimensional Models and Computational Optimization of a Hovering Dragonfly Wing 87

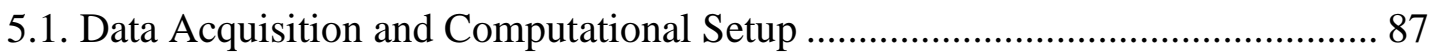

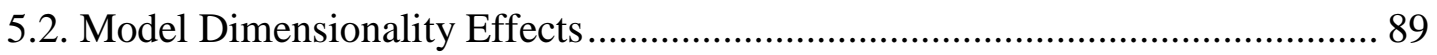

5.2.1. Effect of Model Dimensionality on Aerodynamic Performances................ 90

5.3.2. Effect of Model Dimensionality on Vortex Structure............................... 93

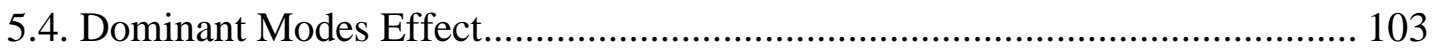

5.4.1. Effect of SSVD Mode 1 and Mode 2 Amplitudes .................................. 105

5.4.2. Effect of Phase Difference between SSVD Mode 1 and Mode 2 .............. 108

5.5. Optimal Low Dimensional Models ............................................................. 109

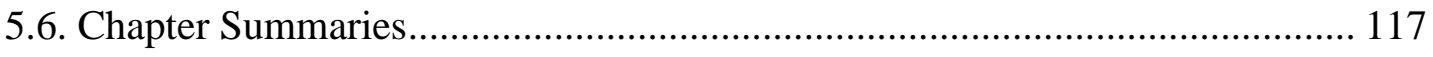

6. The Effect of Chordwise and Spanwise Flexibility on the Hydrodynamic Performance of Cetacean Propulsors ................................................................. 118

6.1. Singular Value Decomposition for morphing kinematics analysis of orca fluke

6.2. Effect of Spanwise and Chordwise Morphing on the Hydrodynamic Performance

6.3. Effect of Spanwise and Chordwise Morphing on the Wake Structure

6.4. Chapter Summaries.

7. Asymmetric Three-dimensional Wake and Aerodynamic Forces of a Maneuvering Hummingbird .

7.1. Data Acquisition

7.2. Computational Setup

7.3. Body and Wing Kinematics.

7.4. Aerodynamic Force 


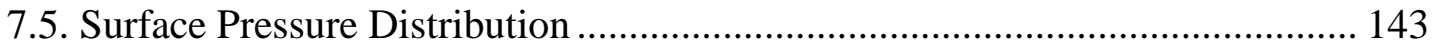

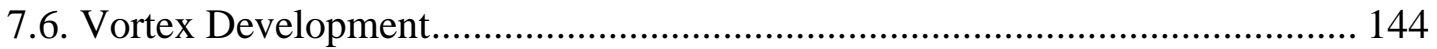

7.7. Near Wake Structure and Dual-Ring Wake Structure .................................... 148

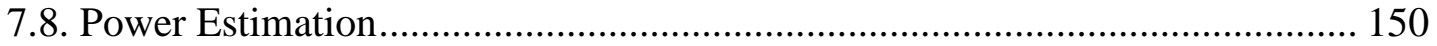

7.9. Chapter Summaries............................................................................. 151

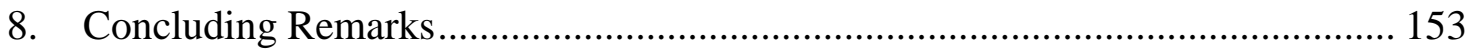

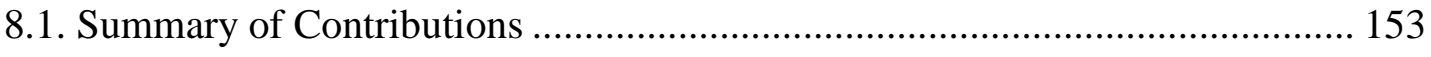

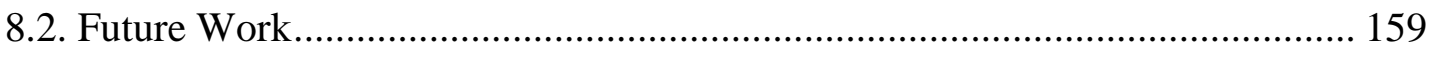

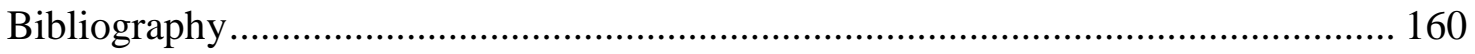




\section{List of Figures}

Figure 1-1. Nature's morphing propulsors/bodies. (a) Hummingbird in maneuvering motion; (b) bat cambering its wings in flight; (c) a dragonfly twisting its wings during flight; (d) a highly deformed bluegill sunfish pectoral fin; (e) spanwise morphing of an orca's fluke; (f) chordwise morphing of an orca's fluke. 2

Figure 1-2. Flow chart of the integrated methodology to systematically study biological propulsor morphing kinematics effect. 11

Figure 2-1. (a) Static model of dolphin with virtual joints; (b) joints configuration at the dolphin fluke; (c) virtual joints configuration based on real dolphin skeletal anatomy. .. 18 Figure 2-2. Schematic plot showing virtual cameras configuration of the dolphin in fast swimming. 20

Figure 2-3. Virtual camera scenes and corresponding images of real cameras for the (a) side camera and the (b) offset camera. The time instance is at $\mathrm{t} / \mathrm{T}=0.6$. 21

Figure 2-4. (a) Experimental setup; (b) reconstructed model based on three orthogonal projected high-speed images; (c) raw image of a hovering dragonfly; (d) schematic plots showing virtual joints configuration of the dragonfly wings. 23

Figure 2-5. (a) Schematic plot of wing kinematics parameters definition; (b) time course of forewing kinematics measurements from the joint-based and the conventional method; (c) time course of forewing deformation measurements from the joint-based and the conventional method. The deformation parameters are measured at mid chord of the wing.

Figure 2-6. Reconstructed model of a takeoff maneuver dragonfly in global (a) and local (b) coordinate system. Colored curves are wing tip trajectories. 27

Figure 2-7. The SSVD spectrum of the first ten modes for the dragonfly right forewing kinematics. The left ordinate shows $\left|\lambda_{i}\right| / \sum\left|\lambda_{i}\right|$, and the right ordinate shows the cumulative value of the left ordinate. 31

Figure 2-8. Wing motions of (a) experimental kinematics (also called the mode-all case), (b) SSVD mode 1, (c) mode 2 and (d) low-dimensional model mode $1+2$. The wings are 
colored with distances between wing surfaces and corresponding least square planes. The distances are normalized by wing mid chord length.

Figure 2-9. Motion percentage histories of low-dimensional models (mode 1+2) for different snapshot sizes. 35

Figure 2-10. Schematic describing the naming convention and location of velocity components employed in the spatial discretization of the governing equations.

Figure 2-11. (a) Example of a baby trout model with unstructured surface mesh of triangular elements; (b) representative example showing the baby trout immersed in a nonuniform Cartesian grid.

Figure 2-12. (a) Cell identification in a normal situation using the conventional method; (b) cell identification in a sharp edge situation using the conventional method; (c) cell identification in a sharp edge situation using the current method.

Figure 2-13. 2D schematic describing the stair boundary and ghost-cell methodology used in the current solver. 47

Figure 2-14. 2D schematic describing the methodology which handles deformable attaching bodies.

Figure 2-15. 2D schematic describing the methodology which handles very close solid body and membrane body. 51

Figure 2-16. Comparison of the baby trout motion shown in high-speed images to the corresponding reconstructed model. (a) Lateral view; (b) ventral view; (c) back view... 53 Figure 2-17. Time course of hydrodynamic (a) thrust and (b) power coefficient of the steady swimming baby trout.

Figure 2-18. Three-dimensional wake structures of the steady swimming baby trout. (a) Lateral view; (b) ventral view; (c) perspective view. 55

Figure 2-19. A schematic of the computational optimization frame. 57

Figure 2-20. Parametric study results showing the effect of trail-edge flap deflection amplitude $\beta$ and the corresponding phase difference $\varphi$ on the aerodynamic lift production. The optimization convergence histories are also shown for comparison...... 62

Figure 3-1. Schematic plot showing the modeling of the flexible wing. 65

Figure 3-2. Convergence histories of design variables (a) $P_{R}$, (b) $\beta_{m}$, and (c) $\varphi$ of case Opt $C_{T}$. 67 
Figure 3-3. Convergence histories of design variables (a) $P_{R}$, (b) $\beta_{m}$, and (c) $\varphi$ of case Opt $\eta$.

Figure 3-4. Time courses (a) thrust coefficients and (b) power coefficients for the cases Opt $C_{T}$, Opt $\eta$, and Rigid. 68

Figure 3-5. Wake structures of the cases (a) Opt $C_{T}$, (b) Opt $\eta$, and (c) Rigid. The contours show the normalized vorticity. 70

Figure 3-6. Mean flows of the cases (a) Opt $C_{T}$, (b) Opt $\eta$, and (c) Rigid. The incoming flow velocity is subtracted from the mean flows. 71

Figure 4-1. Schematic plot showing the definitions of plate kinematics...................... 76

Figure 4-2. Schematic plot showing the modeling of plate twisting. ........................... 77

Figure 4-3. Stretching grids configuration used in the current work........................... 79

Figure 4-4. Thrust (a) and power (b) coefficient of the pitching-rolling plate with different pitching amplitudes (from $15^{\circ}$ to $60^{\circ}$ ). 80

Figure 4-5. Flow structure of a rigid pitching-rolling plate (pitching amplitude is $30^{\circ}$ ) at mid-downstroke. (a) contour of $\omega_{z}$ on the vertical cut slice at 2/3 span away from the root; (b) perspective view of the 3D flow. The vortical structures are identified by the iso-surface of Q-criteria $(\mathrm{Q}=1.0)$. 82

Figure 4-6. Thrust (a) and power (b) coefficient histories of the pitching-rolling plates with optimized dynamic twisting. Results of a completely rigid plate (case Rigid) are also shown for comparison.

Figure 4-7. Side view of the flow structures of (a) case Opt $C_{T}$ and (b) Opt $\eta$. Mean flow (incoming flow subtracted) contour of (c) case Opt $C_{T}$ and (d) Opt $\eta \ldots$ 85

Figure 5-1. The constructed realistic wing-body model immersed in the three-dimensional non-uniform Cartesian grid. 88

Figure 5-2. Comparison of the time course of aerodynamic performances between the Mode-all and low-dimensional gaits. (a) lift; (b) drag; (c) power. 92

Figure 5-3. Wake structures for the (a) mode-all, (b) mode 1, (c) mode 1+2 and (d) mode $1+2+3$ cases. The associate time instance is $t / T=0.25$ 
Figure 5-4. Wake structures for the (a) mode-all, (b) mode 1, (c) mode 1+2 and (d) mode $1+2+3$ cases. The associate time instance is $\mathrm{t} / \mathrm{T}=0.5$. 96

Figure 5-5. Wake structures for the (a) mode-all, (b) mode 1, (c) mode 1+2 and (d) mode $1+2+3$ cases. The associate time instance is $\mathrm{t} / \mathrm{T}=1.0$.

Figure 5-6. 2D slices of the leading edge vortex along the wingspan (10\% 90\%) at two time instances for $(\mathrm{a}, \mathrm{b})$ mode-all, $(\mathrm{c}, \mathrm{d})$ mode $1,(\mathrm{e}, \mathrm{f})$ mode $1+2$ and $(\mathrm{g}, \mathrm{h})$ mode $1+2+3$ cases. The corresponding vortex center are marked with green dots at each slice. The contours represent normalized span-wise vorticities. 99

Figure 5-7. Distances of LEV center to the wing surface (lift-off distances) at (a) $t / T=0.25$ and (b) $\mathrm{t} / \mathrm{T}=0.75$ for mode-all, mode 1 , mode $1+2$ and mode $1+2+3$ cases. 101

Figure 5-8. LEV circulations at (a) $t / T=0.25$ and (b) $t / T=0.75$ for mode-all, mode 1 , mode $1+2$ and mode $1+2+3$ cases

Figure 5-9. Comparison of the time course of aerodynamic performance for the wing gaits with different values of $W_{1}$, while $W_{2}=1.0$. (a) Lift; (b) power. 106

Figure 5-10. Comparison of the time course of aerodynamic performance for the wing gaits with different values of $W_{2}$, while $W_{1}=1.0$. (a) Lift; (b) power. 106

Figure 5-11. Cycle averaged aerodynamic performance of wing gaits with different weight of SSVD Mode 1 and Mode 2. One weight coefficient ranges from 0.5 to 1.5 while another equals to 1.0. (a) Lift; (b) power; (c) lift efficiency.

Figure 5-12. Comparison of the time course of aerodynamic performance for the wing gaits with different phase difference between SSVD Mode 1 and Mode 2. (a) Lift; (b) power. 108

Figure 5-13. Cycle averaged aerodynamic performance of wing gaits with different phase difference between SSVD Mode 1 and Mode 2. (a) Lift; (b) power; (c) lift efficiency. 109 Figure 5-14. Convergence history for design variables (a) $W_{1}$, (b) $W_{2}$, and (c) $\varphi$ of the two optimization cases.

Figure 5-15. Comparison of the time course of aerodynamic performance of Mode $1+2$ and optimized wing gaits. (a) Lift; (b) power. 111

Figure 5-16. Time averaged lift and power coefficients projected on the wing surface for the cases (a, b) Mode $1+2,(\mathrm{c}, \mathrm{d})$ Opt $C_{L}$, and (e, f) Opt $\eta$. 113 
Figure 5-17. Wake structures for the (a) Mode $1+2$, (c) Opt $C_{L}$, (e) Opt $\eta$ at $\mathrm{t} / \mathrm{T}=0.27$; wake structures for the (b) Mode 1+2, (d) Opt $C_{L}$, (f) Opt $\eta$ at t/T=0.73

Figure 5-18. LEV structures for the (a) Mode $1+2$, (c) Opt $C_{L}$, (e) Opt $\eta$ at $\mathrm{t} / \mathrm{T}=0.27$; LEV structures for the (b) Mode $1+2$, (d) Opt $C_{L}$, (f) Opt $\eta$ at t/T=0.73.

Figure 5-19. TV structures for the (a) Mode $1+2$, (c) Opt $C_{L}$, (e) Opt $\eta$ at $\mathrm{t} / \mathrm{T}=0.27$; TV structures for the (b) Mode 1+2, (d) Opt $C_{L}$, (f) Opt $\eta$ at t/T=0.73.

Figure 6-1. The SVD spectrum of the first ten modes for the orca fluke morphing kinematics. The left axis shows the normlized singular values, and the right axis shows the corresponding cumulative values.

Figure 6-2. Time course of the (a) thrust and (b) power coefficient for the five computational models.

Figure 6-3. Near wake structures of (a) case $\mathrm{C}$ and (b) case $\mathrm{SC}$ at $\mathrm{t} / \mathrm{T}=58$, the fluke surfaces are colored by $C_{T}$; near wake structures of (c) case $\mathrm{S}$ and (d) case $\mathrm{SC}$ at $\mathrm{t} / \mathrm{T}=0.25$, the fluke surfaces are colored by $C_{P W}$. The wake structures are colored by spanwise vorticity... 123 Figure 6-4. Far wake structures at $\mathrm{t} / \mathrm{T}=1.0$ of the (a) case $\mathrm{SC}$, (b) case $\mathrm{S}$, and (c) case $\mathrm{C}$; the corresponding 2D flow slices at mid span of the fluke for (d) case SC, (e) case S, and (f) case C. The 2D flow slices are colored by spanwise vorticity and the arrows are vector field of the fluid velocity.....

Figure 6-5. Iso-surface plot of the pressure field at $t / T=1.0$ of the (a) case $S C$, (b) case $S$, and (c) case C; the corresponding schematic plots of wake skeleton for (d) case SC, (e) case $\mathrm{S}$, and (f) case $\mathrm{C}$.

Figure 7-1. Raw pictures of real hummingbird and its reconstructed model at (a, d) $t=0 \mathrm{~ms}$, (b, e) $\mathrm{t}=47 \mathrm{~ms}$, and (c, f) $\mathrm{t}=77 \mathrm{~ms}$.

Figure 7-2. Schematic of the computational mesh and boundary conditions employed in the current simulation. 135

Figure 7-3. (a) Schematic plot showing hummingbird body yaw motion; (b) time course of body of the maneuvering hummingbird. A top view of the hummingbird at the top of the figure indicates the yaw throughout the maneuver. Yaw (red), pitch (black), and roll 
(blue) histories are shown first, followed by path position histories in horizontal (green) and vertical (black) direction.

Figure 7-4. (a) Schematic plot showing hummingbird wing Euler angles definition; Time course of wing stroke (b), wing deviation (c), and wing pitch angles (d) during the turning phase. The wing Euler angle histories over the 3rd stroke are shown in solid curves. Angle differences for other strokes in the turning phase with respect to the 3rd stroke are shown as error bars. Red and blue correspond to the inner and outer wings, respectively. Shaded areas stand the downstrokes and unshaded areas stand the upstrokes.

Figure 7-5. Time course of lift (a) and drag (b) coefficients during the turning phase of hummingbird pure yaw turn. The force coefficient histories over the 3rd stroke are shown in solid curves. Force coefficient differences for other strokes in the turning phase with respect to the 3rd stroke are shown as error bars. Red and blue correspond to the inner and outer wings, respectively. Shaded areas stand the downstrokes and unshaded areas stand the upstrokes.

Figure 7-6. Time-averaged surface pressure distribution projected on the lift direction (a, b) and drag direction (c, d); (a) and (c) correspond to the average over all downstrokes in turning phase and (b) and (d) correspond to the average over all upstrokes in turning phase.

Figure 7-7. (a-f) Time course of vortex development of the 3rd stroke of the hummingbird pure yaw turn, visualized by the Q-criterion. The vortex structures are colored by nondimensional pressure. 146

Figure 7-8. (a) Schematic plot showing slice locations; velocity vector field plots of (b) the inner side slice and (c) the outer side slice. (b) and (c) are colored by vorticity in the direction of the normal of the slice planes. 148

Figure 7-9. (a) Isosurface of pressure corresponding to a non-dimensional value of -1.0 at $\mathrm{t} / \mathrm{T}=0.37$ of the $3 \mathrm{rd}$ stroke of the hummingbird pure yaw turn; (b) wake schematic at $\mathrm{t} / \mathrm{T}=0.37$ of the $3 \mathrm{rd}$ stroke of the hummingbird pure yaw turn. 150

Figure 7-10. Average instantaneous specific power of in the turning phase of the hummingbird maneuvering flight. Both inner wing (a) and outer wing (b) are shown.. 151 


\section{List of Tables}

Table 2-1. Dimensions of the fast swimming dolphin 19

Table 2-2. Camera parameters in the case of fast swimming dolphin

Table 2-3. Averaged motion percentages $\bar{\varepsilon}$ and normalized singular values $\lambda^{*}$ of lowdimensional models (mode 1+2) for different snapshot sizes.

Table 2-4. SSVD analysis of several flapping propulsors in nature.

Table 2-5. Baby trout steady swimming parameters...... 52

Table 2-6. Cycle averaged hydrodynamic performance 55

Table 3-1. Cycle averaged aerodynamic performance. 69

Table 4-1. Pitching-rolling plate cases without surface morphing. 78

Table 4-2. Time averaged value of $C_{T}, C_{P W}$, and $C_{T} / C_{P W}$ of rigid pitching-rolling plate during the fifth flapping cycle.

Table 4-3. Time averaged value of $C_{T}, C_{P W}$, and $C_{T} / C_{P W}$ of rigid pitching-rolling plate with optimized dynamic twisting. Results of a completely rigid plate (case Rigid) are also shown for comparison.

Table 5-1. Cycle averaged aerodynamic performance of the mode-all and low-dimensional gaits. 93

Table 5-2. Cycle averaged hydrodynamic performance of Mode $1+2$ and optimized wing gaits.

Table 6-1. Cycle averaged hydrodynamic performance of the five computational models.

Table 7-1. Morphological data for the calliope hummingbird.

Table 7-2. Half cycle averaged wing angle of attack for the inner and outer wings of the maneuvering hummingbird. 141 


\section{Introduction}

\subsection{Background and Motivation}

In nature, flying and swimming are unique mechanisms for generating control and maneuvering forces, and these forces are primarily generated by flapping wings or fins. Flapping propulsion offers an attractive alternative to conventional propulsion methods for future bio-inspired aerial/underwater systems. Weis-Fogh and Jensen (1056) [1, 2] described flapping propulsion as a complex physical and biological problem, which is so complex that it is impossible to understand a single part of the process. One of the reasons for this is the essential complexity in unsteady motion of propulsors and the related flow mechanisms in a Reynolds number of 10 to $10^{5}$ [3]. Within this flow regime, lift/thrust producing mechanisms are intrinsically unsteady and vortex-dominated. Throughout the years, progress in understanding flapping propulsion has been accomplished and a general understanding of the unsteady mechanisms has been identified [4-33] such as clap-andfling [12, 16, 34-36], leading-edge vortex [7, 37-39], rotational lift [7, 40], and wake capture [7, 41], etc. By taking the rigid propulsor assumption, these mechanisms have helped explain the fluid phenomena near the propulsor, which are clearly expressed as the motion of vortices, as well as the instantaneously local and resultant forces.

However, most of nature's creatures that can fly or swim are equipped with flexible or deformable propulsors. Among them, birds can twist and bend their wings to change flight course or adjust to wind gusts. For example, hummingbirds can actively camber and twist their wings during hovering and maneuvering flights [42, 43] (Figure 1-1a). Bats can also camber and morph their wings through their independently controlled wing joints, highly 
deformable bones, and compliant wing membrane [44] (Figure 1-1b). Four-winged flyers, dragonflies, also flex their wings during the flights [45] (Figure 1-1c). Bluegill sunfishes demonstrate significant dynamic morphing of their pectoral fins during the swimming [46] (Figure 1-1d). The morphing is largely caused by fin flexural stiffness, kinematics, and fluid-structure interactions. Marine mammals, orcas, show great span and chordwise morphing of their flukes during steady swimming [47] (Figure 1-1e and f). These flapping propulsors demonstrate not only large three-dimensional deformations, but also elaborate three degree-of-freedom (3-DOF) wing kinematics in space [48]. Therefore, understanding the aero/hydrodynamic role of propulsor morphing kinematics is critical for the future bioinspired propulsor design.
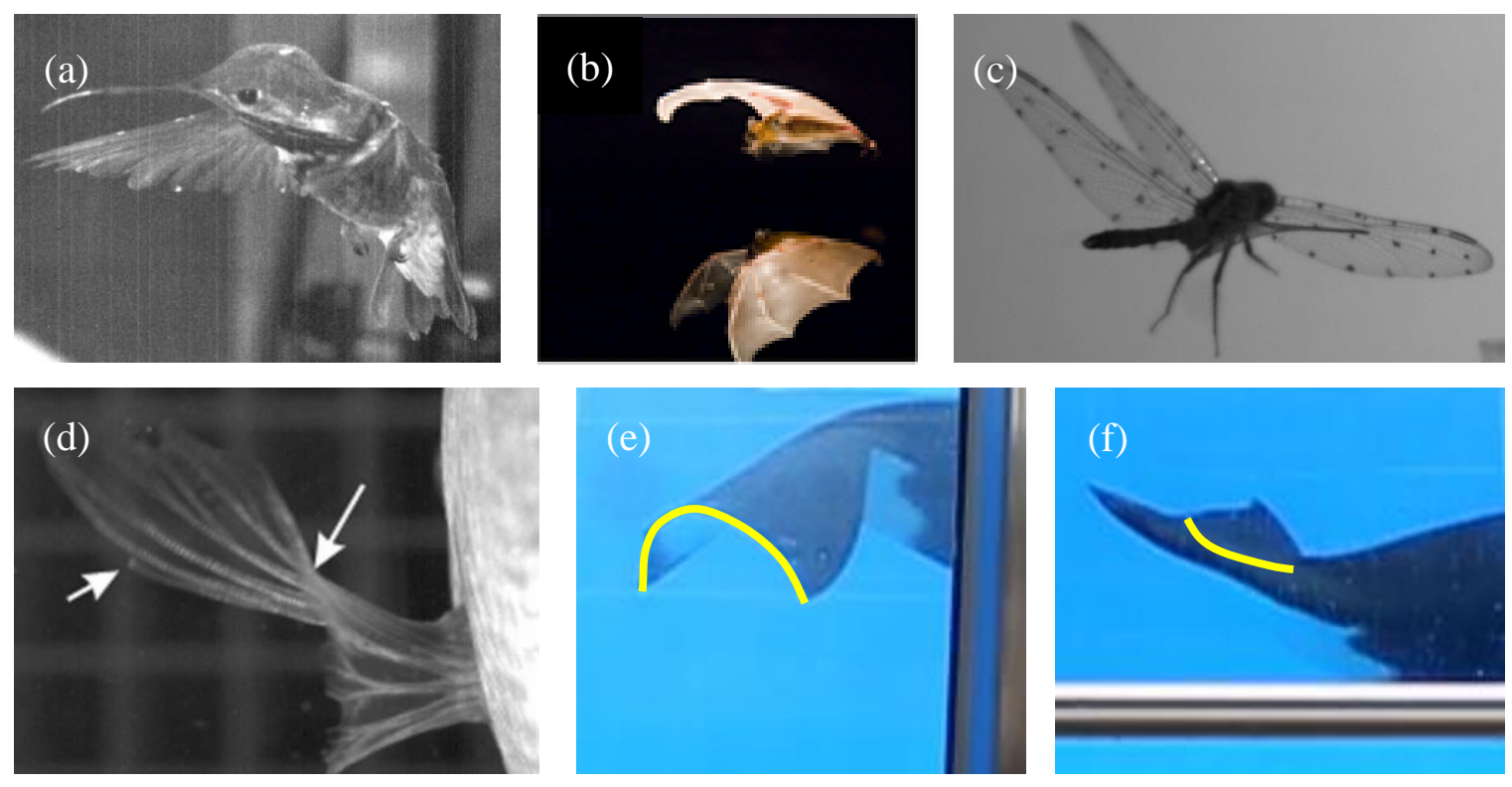

Figure 1-1. Nature's morphing propulsors/bodies. (a) Hummingbird in maneuvering motion; (b) bat cambering its wings in flight; (c) a dragonfly twisting its wings during flight; (d) a highly deformed bluegill sunfish pectoral fin; (e) spanwise morphing of an orca's fluke; (f) chordwise morphing of an orca's fluke. 


\subsection{Data Acquisition}

More recently, advanced photogrammetry technology has been used to study flapping wing flight, which is capable of measuring detailed wing kinematics, as well as deformation even in the wings of tiny flyers like insects. Liu and Sun used three orthogonally aligned high-speed cameras to study the wing kinematics and aerodynamics of hovering droneflies [49]. Walker and colleagues used a four-camera photogrammetric system and a combination of manual and semi-automatic tracking of natural features and marker points to reconstruct the wing kinematics and surface topographies of free-flying hoverflies and tethered locusts [50]. Koehler and colleagues presented a unified methodology for the reconstruction and analysis of the deformations and surface morphology exhibited by insect wings during free flight [51]. They successfully eliminated all rigid wing assumptions during the reconstruction and deformation analysis while minimizing the number of tracked points in the output of the photogrammetry system. However, the reconstruction based on marker points on the wing is still time-consuming, which compromises efficiency, and it has several hard constraints regarding some details of the experiments, such as the camera location/orientation, the lens angle of view, marker points on the objects, etc. For example, it is usually difficult for us to configure three orthogonal cameras outside of the lab, and much of the bio-propulsor locomotion data generated by biologists was not taken in the lab. For some large animals, such as dolphin and orca, it is not possible for us to use a lens with a small angle of view due to the animal's size and movement. Perspective error can become a big issue in those cases. Moreover, placing marker points on the propulsor could prove difficult due to the propulsor's size or 
property. A more robust reconstruction method is needed to deal with diverse flapping propulsors in nature.

\subsection{Effect of Propulsor Morphing Kinematics}

It is widely thought that propulsor morphing would potentially provide new aero/hydrodynamic mechanisms of force productions over completely rigid propulsors in flight/swimming [52-57]. Several studies have demonstrated that the unsteady aerodynamic phenomena of the flapping mechanism are not only sensitive to variations in the wing kinematics but also to the wing's morphing $[58,59]$. Results also revealed that a dynamically adjustable wing surface may potentially provide new aerodynamic mechanisms $[60,61]$ of force production over fully rigid wings $[7,62]$ in flapping flight. Further research $[63,64]$ has illustrated that the performance of a rigid flapping wing can be improved significantly by adding some level of flexibility to the wing surface. For achieving such performance enhancement, both passive and active flow control mechanisms have been studied, and some examples are reviewed here.

In order to understand the effects of chordwise flexibility on the aerodynamics of flapping motion, a model with a hinge connecting two rigid components is commonly used because of its simplicity and preservation of the flexibility characteristics in the chordwise direction [65]. Through this passive deformable model, Eldredge et al. [66] revealed that wings with moderate flexibility have better power efficiency compared to the rigid wing in hovering flight, nevertheless very flexible wings will degrade the overall aerodynamic performance. Using a similar model, Vanella et al. [67] showed that if parameters are 
chosen appropriately, the chord-wise flexibility can result in an enhancement of up to $28 \%$ in the lift-to-drag ratio and a 39\% increase in the lift-to-power ratio over a rigid plate. Wan et al. [68] studied the effect of chord-wise flexibility over a range of hovering kinematic parameters using a hinged-plate model. Their results indicated that the maximum lift-todrag ratio can be achieved by placing the hinge at the three-quarter chord position from the leading edge.

In addition to passive mechanisms explorations mentioned above, active flow control via wing surface morphing has been widely adopted in both fixed-wing and rotary-wing aircraft design. These include articulated flaps and/or slats [69], surface flow control devices [70] and continuously deforming surfaces [71]. Among them, the flow control via trailing-edge flap (TEF) is presumed to be more applicable to novel flapping-wing MAVs designs, in terms of simplicity of moving surface control, weight of MAVs and so on. Liu et al. [72] experimentally studied the effect of actively controlled trailing-edge flaps on the flow control of translational plates. Their results have shown that force and flow characteristics strongly depend on the timing of trailing-edge flap deflection rather than translational speed. Li et al. [73] and Xu et al. [74] further studied the effect of the trailingedge flap on the aerodynamic performance of a 2-D flapping wing via a computational approach. It is found that the aerodynamic forces can be significantly affected by controlling the timing of the flap deflection in a flapping cycle.

With the help of advanced photogrammetry and reconstruction technology, the aero/hydrodynamic effects of flapping propulsor morphing kinematics are studied in recent years. Young and colleagues computationally studied the wing camber and twist effects of a tethered desert locust [75]. They came up with 'uncambered' models from the full-fidelity 
model (originally reconstructed wing model) by replacing the cambered chord with a straight line connecting the leading and trailing edge (hindwing) or a straight line at the same mean incidence (forewing). An 'untwisted' model of forewing was also constructed by removing torsional deformation along the span, replacing the forewing with a flat plate of the same instantaneous projected area and same instantaneous angle of attack at the midwing position. The hindwing 'untwisted' model was constructed by replacing the fullfidelity model with two flat plates of the same total instantaneous projected area, joined along a line running from the axilla to a point midway between the fourth and fifth anal veins at the trailing-edge. The aerodynamic features of the uncambered and untwisted model were then compared with the full-fidelity model. They concluded that the fullfidelity model achieved greater power economy than the uncambered mode, which performed better than the untwisted model. However, the simplified wing models (uncambered and untwisted models) were constructed based on empirical measurements. Different ways of subtracting wing morphing may lead to different conclusions. A more solid and robust approach for the analysis of propulsor morphing kinematics is needed.

\subsection{Singular Value Decomposition (SVD)}

To better analyze the complex propulsor morphing kinematics, singular value decomposition (SVD) was used previously to decompose the motions of a highly deformed fish pectoral fin used by a bluegill sunfish during steady forward swimming [76]. SVD is a powerful method for data analysis, aimed at obtaining low-dimensional, approximate descriptions of a high-dimensional process or dataset [77, 78]. The SVD or similar methods, 
such as proper orthogonal decomposition (POD) and principle-component analysis (PCA), have been used in many areas including image processing, data compression, process identification and oceanography [77]. POD has also been used to obtain approximate, lowdimensional descriptions of turbulent flows [79], structural vibrations, and dynamical systems. PCA has been used before for understanding the gait of biological entities [80]. Bozkurttas and colleagues have successfully applied SVD in their work and found out that the complex fin motion is dominated by a relatively small number of SVD modes (five modes) [76]. The first three dominant modes, which contain $67 \%$ of the total motion, can recover $92 \%$ of the thrust of the pectoral fin. Moreover, the mode 3, which is a wave-like motion in the span-wise direction, is primarily a result of flow-induced deformation. The SVD analysis suggests one natural approach to the development of the robotic propulsors. Since a small number of modes capture a significant portion of the motion, it stands to reason that a systematic procedure to developing a robotic propulsor would to be to design actuation mechanisms that reproduce a small number of these modes.

\subsection{Optimum Design of Flapping Propulsors}

The development of flapping propulsion systems in recent years has been inspired primarily by flying/swimming animals. Design of such systems usually follows a 'bioinspired' approach where key features of the biological system are identified, their function understood and the feature then modified and/or simplified for adoption into the engineered system. This approach is based on the recognition that an engineered system usually has design objectives that differ from those of the biological flyers/swimmers. Biological 
organisms are a result of evolution through natural selection, which does not necessarily produce designs that are 'optimal' in terms of performance metrics relevant to a designer. The ultimate objective of any biological organism is to successfully reproduce and pass along its genetic information to subsequent generations. This may be accomplished in a multitude of ways, some of which are detrimental to improved flight performance. For example, once adequate flight performance is achieved, animals may evolve to direct resources toward reproduction rather than further improvements in flight ability. In contrast, an engineered flapping propulsion system might have more specific design objectives in terms of range, payload, etc. In addition, evolution by natural selection is a dynamic process which is constantly affected by an ever-changing environment, and biological designs often include vestigial or redundant features that increase complexity without enhancing performance. This implies the possibility of learning from biological systems and then subsequently simplifying their design and/or improving on their performance.

The bio-inspired design approach requires tools that can evaluate candidate designs and search for optimal (or at least higher-performing) solutions. Evaluation and optimization through experiments is a possibility, but can be prohibitively expensive given the dimensionality and size of the parameter space that often needs to be explored. Computational optimization seems to be well suited for this purpose, but here too, one has to compromise between model fidelity and computational effort, especially for 3D cases. Several optimization attempts based on simplified 2D flapping propulsors can be found in the literatures [81, 82]. For 3D bio-inspired propulsor optimum design, Zheng et al. used low fidelity BEM model to evaluate the propulsor aerodynamic performance when searching within the design space. 
Design optimization based on low dimensional models of biological propulsion systems suggests one natural approach to the development of the robotic propulsors. The increased complexity of the biological systems due to vestigial or redundant features is reduced in the low dimensional models. The design variables are limited to just a few dominant SVD modes. A gradient-based parallel curve searching optimizer is developed in the current work to best fit with the low dimensional models of biological propulsors. A computational optimization frame, which consists of the low dimensional models, the highfidelity flow solver, and the optimizer, is then built. The computational cost of finding the searching direction and the step size greatly reduced with the help of this frame.

\subsection{Current Objectives}

In summary, the objectives of the current work include two parts: tools development and analysis of flapping propulsion systems. For the tools development part, we have the following four objectives:

\section{Objective 1: Develop a high-accurate kinematics measurement tool to quantify the propulsor flexion and body kinematics of animals in free flight/swimming.}

The conventional reconstruction method [51] has several hard constraints regarding some details of the experiments, such as the camera location/orientation, the lens angle of view, marker points on the objects. A joint-based surface reconstruction method is developed to overcome the above issues. The robustness and efficiency of the modified 
method is improved by adding virtual joints to the propulsor, which can control the propulsor motion in a more efficient way. The accuracy of the method is reserved while the efficiency of the reconstruction is doubled.

\section{Objective 2: Develop a spherical-coordinates-based singular value decomposition (SSVD) tool for understanding complex morphing propulsor kinematics.}

The conventional SVD method [76] has several drawbacks regarding the distinctiveness and physics of the modes. For example, large propulsor morphing can be found in several modes and some modes show great surface area change even though the surface area change in original motion is limited. The new method (SSVD) is developed to overcome the above issues. It is proved to be more compatible with flapping propulsion systems, in which the propulsors are rotating about the propulsor base. The spherical coordinate system can best fit the characteristics of the propulsor motion.

\section{Objective 3: Develop an immersed boundary method for deformable attaching bodies (IBM-DAB) to deal with the moving boundaries in direct numerical simulations.}

The new method is developed to handle direct numerical simulations in some extreme situations that are commonly exist in nature, including solid bodies with sharp edges and those with deformable, attaching membrane bodies. 


\section{Objective 4: Develop a parallel curve searching optimizer for performing computational optimization on flapping propulsors.}

A gradient-based parallel curve searching optimizer is developed in the current work to best fit with the computational optimization of low dimensional models of biological propulsors. A computational optimization frame, which consists of the low dimensional models, the high-fidelity flow solver, and the optimizer, are built. The computational cost of finding the searching direction and the step size is greatly reduced with the help of this frame.

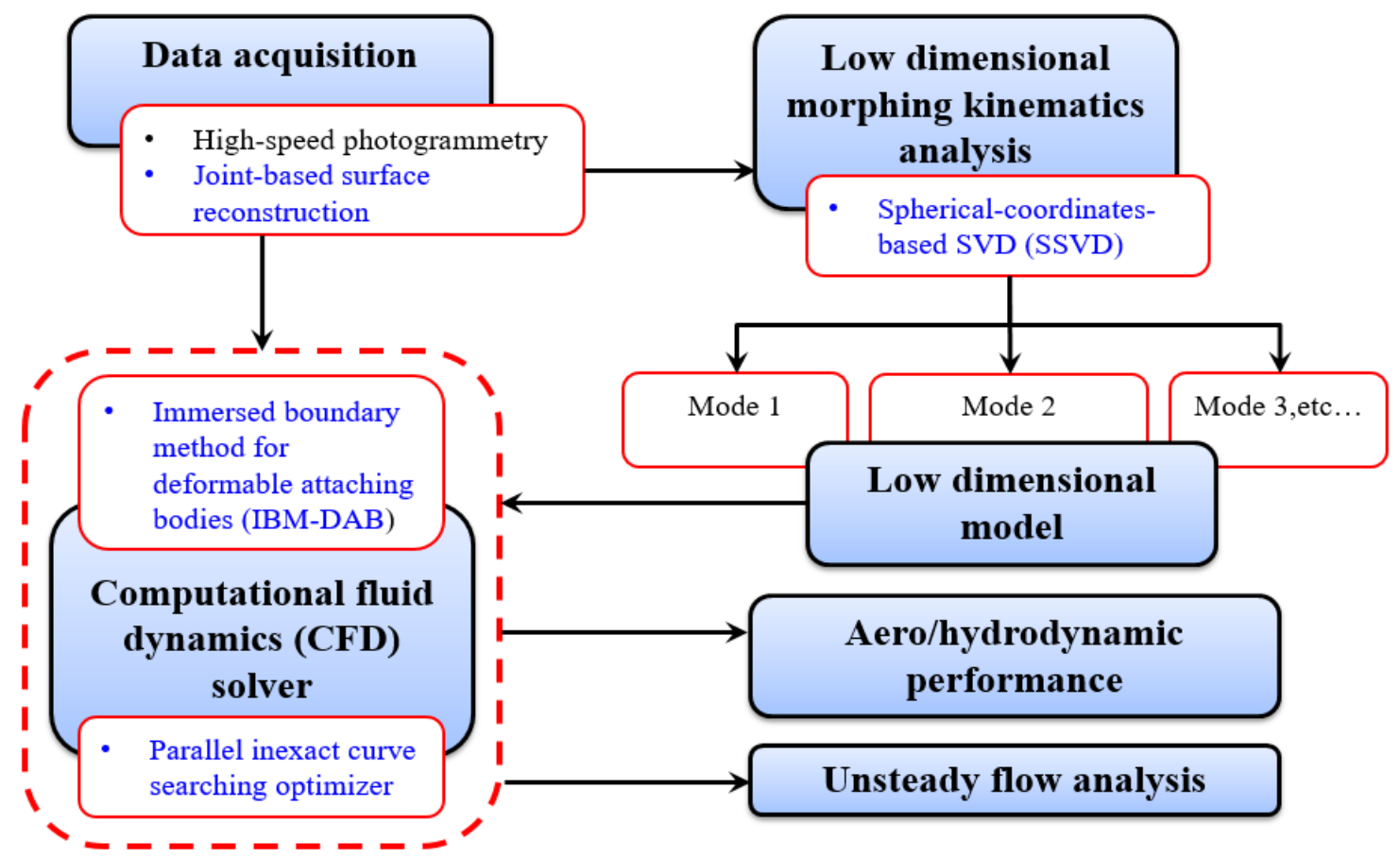

Figure 1-2. Flow chart of the integrated methodology to systematically study biological propulsor morphing kinematics effect. 
The integrated methodology consists of the above tools. Figure 1-2 shows a flow chart of the methodology. High-speed photogrammetry is used to obtain raw data of various natural flyers/swimmers such as dragonfly, hummingbird, trout, and orca. A variety of motions including hover, maneuver, and steady swimming are recorded. After that, the joint-based surface reconstruction technique is used to obtain the detailed kinematical and morphological data. Next, the spherical-coordinates-based SVD (SSVD) method used to decompose the complex wing motions to SSVD modes. We further investigate optimal configurations of dominant modes on aero/hydrodynamic performance for the biological propulsors using the computational optimization frame. The corresponding optimized low dimensional models, which surpass biological levels of aero/hydrodynamic performance, are obtained.

For the part of flapping propulsion systems analysis, we have the following three objectives:

\section{Objective 5: Investigate the optimal dynamic camber formation and dynamic twisting of flapping propulsors via simple canonical model problems.}

In order to test the computational optimization frame and obtain basic sense of the aerodynamic effect of propulsor morphing kinematics, we first investigate the optimal configurations of several morphological parameters that control the dynamic camber and twisting of the propulsors on aerodynamic performance using simplified canonical models. Optimizations of dynamic camber formation of $2 \mathrm{D}$ pitching-plunging plates and dynamic twisting of 3D pitching-rolling plates are performed. 
Objective 6: Study the complex morphing propulsor kinematics and the associated aero/hydrodynamics of natural flyers/swimmers in relatively simple motions.

The integrated methodology is used to study the complex morphing propulsor kinematics and the associated aero/hydrodynamics of a hovering dragonfly and a fast swimming orca. The SSVD analysis is performed on both cases. The model dimensionality and dominant modes effect are studied. For the case of hovering dragonfly, computational optimization cases are performed to find optimal low dimensional models for maximizing the lift production and lift efficiency. Comparative studies on aerodynamic performance and vortex structures are then performed on the optimal cases. For the case of fast swimming orca, the effect of spanwise and chordwise morphing of the fluke are examined. Three low dimensional models (spanwise morphing only, chordwise morphing only, and both spanwise and chordwise morphing) are constructed based on dominant SSVD modes (spanwise morphing mode and chordwise morphing mode) and their hydrodynamic performance and wake structures are compared in detail.

\section{Objective 7: Explore the aerodynamic functions of natural flyers in complex maneuvering flight.}

The integrated methodology is extended to study the most complex propulsion system in nature. Hummingbirds perform turning maneuvers as often as they hover or cruise, especially when they need to forage from one location to another. However, to this date, turning flight had received little attention and most previous studies are primary focused on hovering, forward flight and backward flight, using a variety of techniques. There is no 
data with enough detailed forces, moments and three-dimensional flow structure to achieve a quantitative analysis of a hummingbird in a turning maneuver. To fill this gap, the integrated methodology is used to study the three-dimensional wake structures and aerodynamic forces of a maneuvering hummingbird. To the best of our knowledge, this is the first study on the unsteady aerodynamics of a hummingbird maneuvering in flight.

\subsection{Outline of Thesis}

Chapter 2 describes details of the integrated experimental and computational methodology to systematically study the flapping propulsion system in nature, including the joint-based surface reconstruction method, the spherical-coordinates-based singular value decomposition method (SSVD), the immersed boundary method for deformable attaching bodies (IBM-DAB), the gradient-based parallel curve searching optimization method. The methodologies of Chapter 2 form the basis of the following publication:

- $\quad$ Yan Ren, Haibo Dong, "An Immersed Boundary Method for Deformable Attaching Bodies," Computers \& Fluids, under preparation.

Chapter 3 discusses the computational optimization of dynamic camber formation of 2D pitching-plunging plates. The results of Chapter 3 form the basis of the following publications:

- $\quad$ Yan Ren, Haibo Dong, "Computationa Optimizaiton of Flexible Wing Aerodynamic Performance in Hover," 30 ${ }^{\text {th }}$ AIAA Applied Aerodynamics Conference, 2012. 
- Yan Ren, Haibo Dong, "Computationa Optimizaiton of Flexible Piching-Plunging Plate ," International Journal of Micro Air Vehicles, submitted.

Chapter 4 presents the computational optimization of dynamic twisting of 3D pitching-rolling plates. The results of Chapter 4 form the basis of the following publication:

- Yan Ren, Haibo Dong, "Effect of Surface Morphing on the Wake Structure and Performance of Pitching-rolling Plates," $53^{\text {rd }}$ AIAA Aerospace Science meeting, 2015.

Chapter 5 discusses the low dimensional analysis and aerodynamics of a hovering dragonfly. The results of Chapter 5 form the basis of the following publication:

- Yan Ren, Haibo Dong, "Low-dimensional Modeling and Aerodynamics of Flexible Wings in Flapping Flight," $34^{\text {th }}$ AIAA Applied Aerodynamics Conference, 2016.

Chapter 6 discusses the low dimensional analysis and hydrodynamic effect of spanwise and chordwise morphing of a fast swimming orca fluke. The results of Chapter 6 form the basis of the following publication:

- Yan Ren, Haibo Dong, Frank Fish, "The Effect of Chordwise and Spanwise Flexibility on Hydrodynamic Performance of Cetacean Propulsors," Bioinspiration \& Biomimetic, under preparation.

Chapter 7 presents the results of the three-dimensional wake structures and aerodynamic forces of a maneuvering hummingbird. The results of Chapter 7 form the basis of the following publication: 
- Yan Ren, Haibo Dong, Bret Tolbalske, "Asymmetric Three-dimensional Wake and Aerodynamic Forces of a Maneuvering Hummingbird," Physiccal Review Fluids, under preparation.

Chapter 8 summarizes the conclusions of the dissertation and points toward future work. 


\section{Methodology}

\subsection{Joint-based Surface Reconstruction}

Recently, advanced photogrammetry technology has been used to study flapping propulsors in nature. Along with the highly accurate surface reconstruction method [51], researchers are capable of digitizing detailed propulsor kinematics, as well as deformation from high-speed images. However, the conventional reconstruction method has several hard constraints regarding some details of the experiments, such as the camera location/orientation, the lens angle of view, marker points on the objects, etc. For example, it is usually hard for us to configure three orthogonal cameras outside the lab, and most bio-propulsor locomotion data generated by biologists are not taken in the lab. For some large animals, such as dolphin and orca, it is not possible for us to use lenses with a small angle of view due to their size and movement. Perspective error becomes a big issue in those cases. Moreover, placing marker points on the propulsor could be hard due to the propulsor's size or properties. A more robust reconstruction method is needed to deal with diverse flapping propulsors in nature.

A joint-based surface reconstruction method is developed in Autodesk Maya to solve the above problems in this work. Here we use a case of a fast swimming dolphin to demonstrate the new method. 


\subsubsection{Static Model and Virtual Joints}

In order to perform the reconstruction, a realistic static model of the dolphin with some carefully configured motion controllers, which are so called "virtual joints", is needed at first.

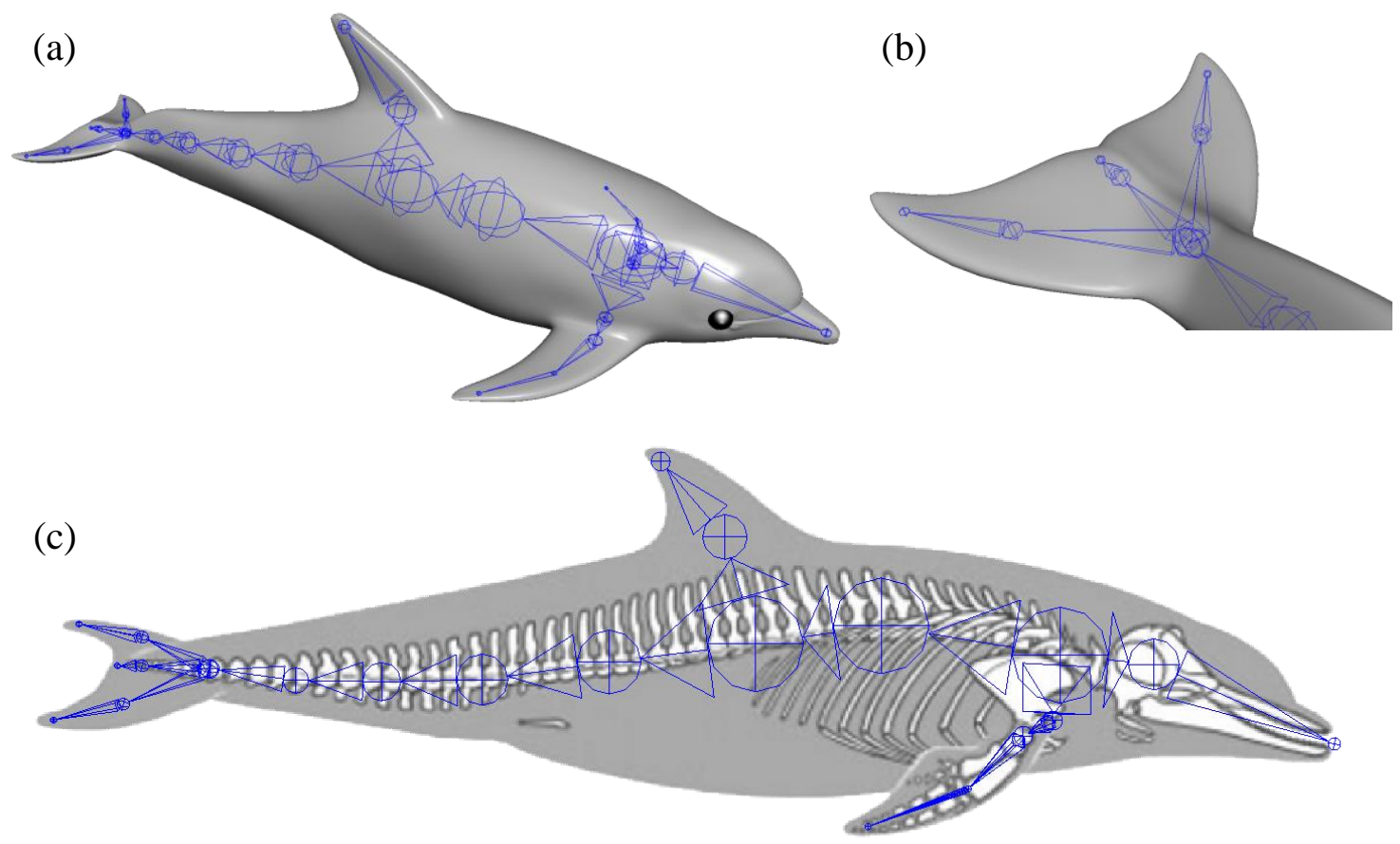

Figure 2-1. (a) Static model of dolphin with virtual joints; (b) joints configuration at the dolphin fluke; (c) virtual joints configuration based on real dolphin skeletal anatomy.

Figure 2-1(a) shows the static model of the dolphin with virtual joints, which is manually created with Autodesk Maya. The dimensions of the model strictly match with the real dolphin we are going to study, and the corresponding values are listed in Table 2-1 in terms of the fluke chord length. The virtual joints have six degrees of freedoms (three translations and three rotations) to control the motion of adjacent vertices of the model. 
Figure 2-1(b) shows the virtual joints configuration at the dolphin fluke, which presents large deformation during the swimming. This kind of configuration can model both the span and chordwise deformation of the fluke. As shown in Figure 2-1(c), the virtual joints are built based on a real dolphin's skeletal anatomy. However, some parts of the dolphin's body, which present large motion during swimming, do not really have bone structures, such as the fluke and the dorsal fin. We also add virtual joints to control the motion for those parts.

Table 2-1. Dimensions of the fast swimming dolphin

\begin{tabular}{ll}
\hline Body length & $12.3 \mathrm{c}$ \\
Body width & $3.0 \mathrm{c}$ \\
Body height & $2.9 \mathrm{c}$ \\
Fluke span & $2.2 \mathrm{c}$ \\
\hline
\end{tabular}

\subsubsection{Virtual Cameras and Reconstruction}

In the experiments, we have several cameras placed around the object to capture its motion. During the reconstruction phase, virtual cameras are created around the static model with Autodesk Maya based on the configurations of the real cameras, including the camera locations/orientations, focal length, angle of view, even the motion of the cameras if they are not static in the experiments. The detailed parameters of the two cameras used to shoot the fast swimming dolphin are listed in Table 2-2. The camera translation is measured based on the location of the static model dolphin. The purpose of creating such virtual cameras is to obtain several $2 \mathrm{D}$ scenes that have the same perspective effects as 
those images taken from real cameras. The perspective error is eliminated by reconstructing the object's motion based on those 2D scenes.

Table 2-2. Camera parameters in the case of fast swimming dolphin

\begin{tabular}{lcccccccc}
\hline \hline Cameras & $T_{x}$ & $T_{y}$ & $T_{z}$ & $R_{x}$ & $R_{y}$ & $R_{z}$ & $\mathrm{AOV}$ & $\mathrm{FL}$ \\
\hline Side & $24.3 \mathrm{c}$ & $-1.9 \mathrm{c}$ & $19.7 \mathrm{c}$ & $-1.8^{\circ}$ & $79.2^{\circ}$ & $0^{\circ}$ & $61.9^{\circ}$ & $30.0 \mathrm{c}$ \\
Offset & $22.3 \mathrm{c}$ & $3.1 \mathrm{c}$ & $40.6 \mathrm{c}$ & $-11.1^{\circ}$ & $45.2^{\circ}$ & $0^{\circ}$ & $61.9^{\circ}$ & $30.0 \mathrm{c}$ \\
\hline
\end{tabular}

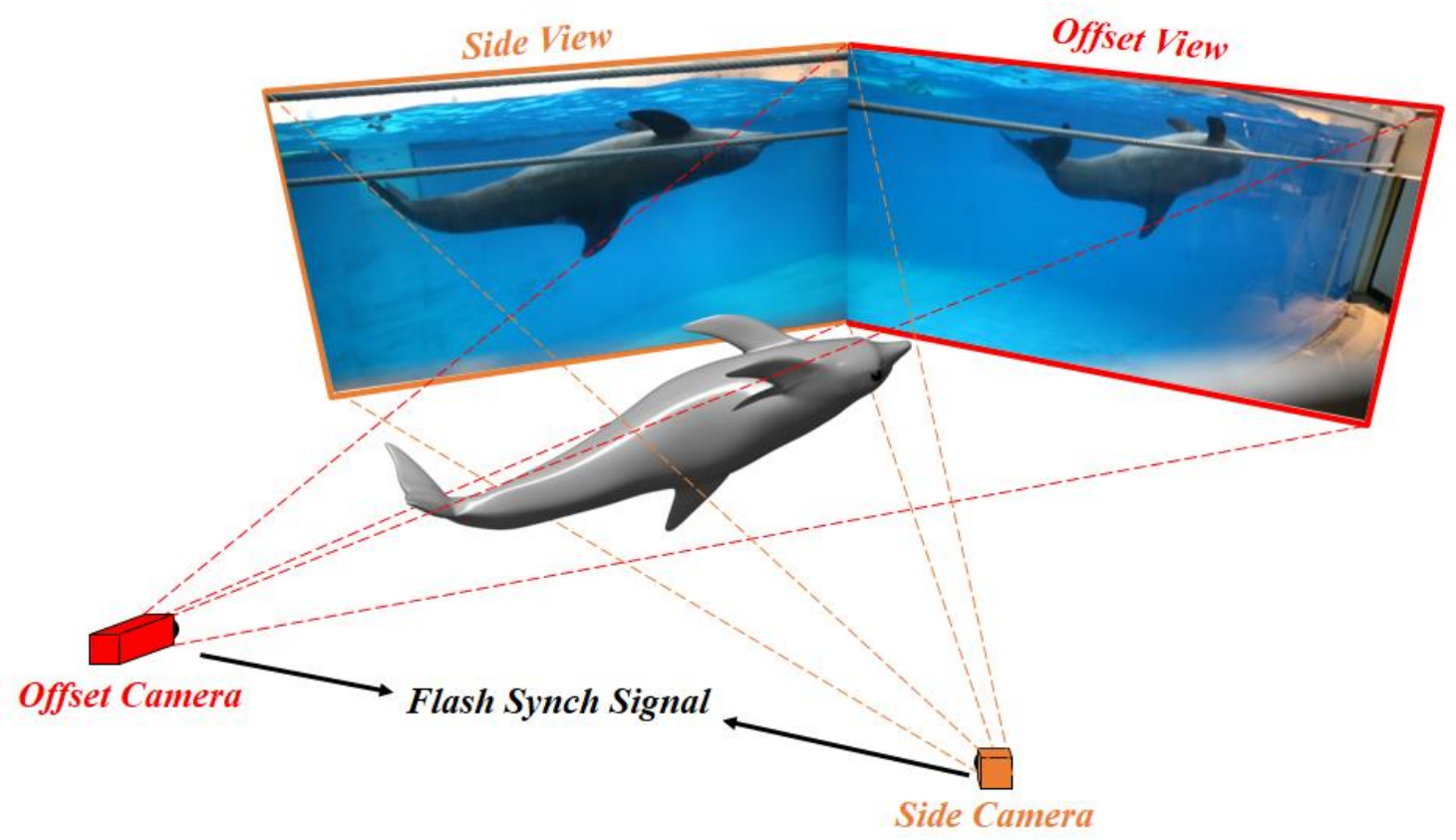

Figure 2-2. Schematic plot showing virtual cameras configuration of the dolphin in fast swimming. 
Figure 2-2 shows a schematic plot of the virtual cameras configuration for the case of the fast swimming dolphin. In this case, we have two virtual cameras, a side camera and an offset camera, which correspond to the side view and offset view of the real cameras, respectively. Two 2D scenes (Figure 2-3) can be obtained by looking though the virtual cameras. Within each 2D scene, we perform the reconstruction by adjusting the motion of the dolphin model via virtual joints to match with the outline of the dolphin in the corresponding views of the real cameras frame by frame until the whole motion of the dolphin is successfully reconstructed.
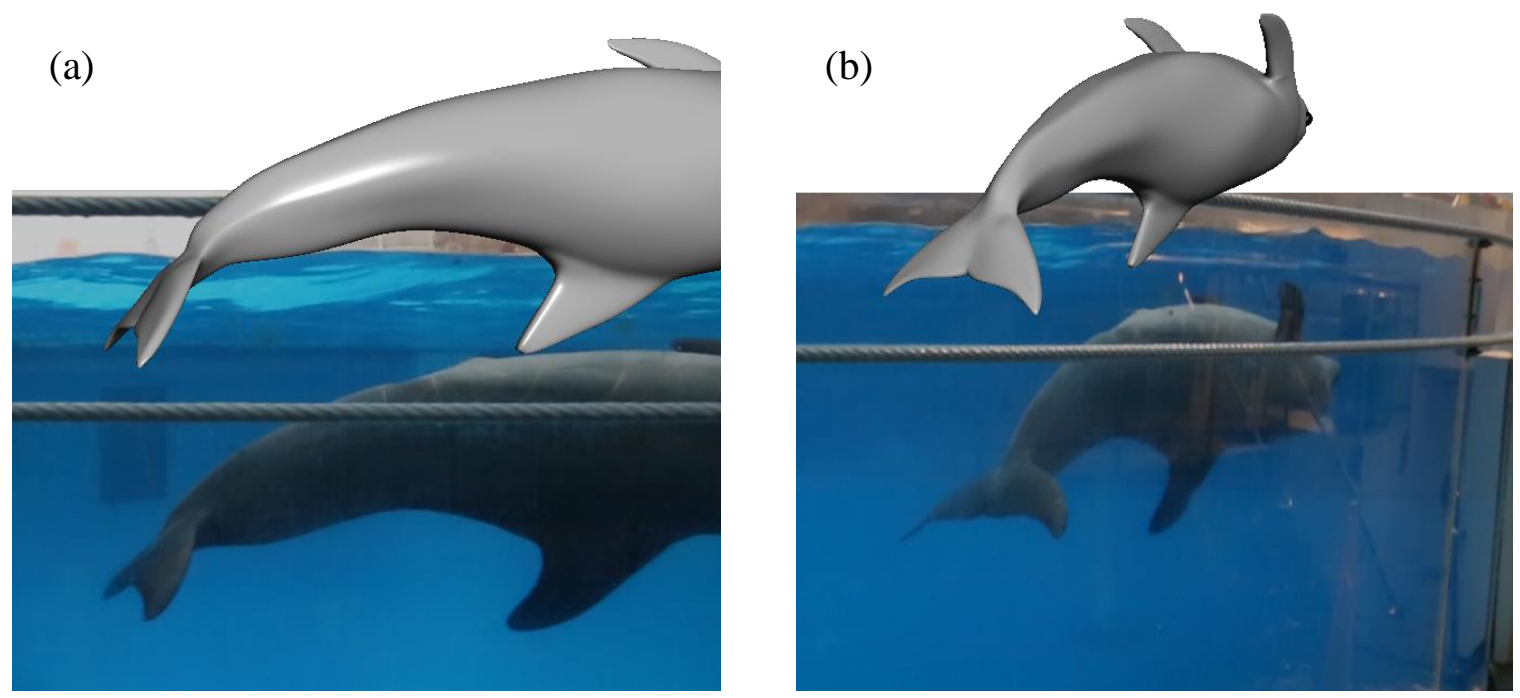

Figure 2-3. Virtual camera scenes and corresponding images of real cameras for the (a) side camera and the (b) offset camera. The time instance is at $\mathrm{t} / \mathrm{T}=0.6$.

\subsubsection{Validation: Deformable Wing Kinematics of a Hovering Dragonfly}

In this section, the forewing motion of a hovering dragonfly is reconstructed using both the conventional reconstruction method [51] and the joint-based reconstruction method. 
The photogrammetry setup in this case consists of three synchronized Photron FASTCAM SA3 60K high-speed cameras (Motion Engineering Company, Indianapolis, IN, USA) with $1024 \times 1024$ pixel resolution (Figure 2-4a). They are aligned orthogonally to each other on an optical table and operated at $1000 \mathrm{~Hz}$ with at least a $1 / 20,000 \mathrm{~s}$ shutter speed to capture the flapping flight videos. The dragonfly is illuminated by two halogen photo-optic lamps (OSRAM, 54428). The cameras are positioned $1.5 \mathrm{~m}$ away from the flying animal, giving a depth of field of 3 to 4 body lengths in all directions depending on the size of the specimen. Given that the dragonfly flaps at approximately $42 \mathrm{~Hz}$, this provides good temporal and spatial resolution.

As shown in Figure 2-4(b), a model dragonfly is built based on three orthogonal projected high-speed images, using the joint-based reconstruction technique. A raw highspeed image of a hovering dragonfly is shown in Figure 2-4(c). Based on the image, we create virtual joints (circled) according to the wing structures. As mentioned before, the virtual joints are used to control the model wing motion until the outline matches with the raw image. Since we have marker points on the wing surface in this case, the surface vertices (purple dots) are then adjusted to match with the marker point. The steps are then performed on other frames until the entire motions of the wings are reconstructed.

Compared to the conventional reconstruction method introduced by Koehler and colleagues [51], the modified method is more efficient, and the accuracy is reserved. As we can see in Figure 2-4(d), the motion handles, which control the wing motions, in the current method are virtual joints and surface vertices, while in conventional method are both edge vertices and surface vertices. In the case shown here, for the left forewing of a hovering dragonfly, the total number of the motion handles in the current method is 10 
while that in the conventional method is 24 . It suggests that the efficiency of the current method is more than twice as much as the conventional one.
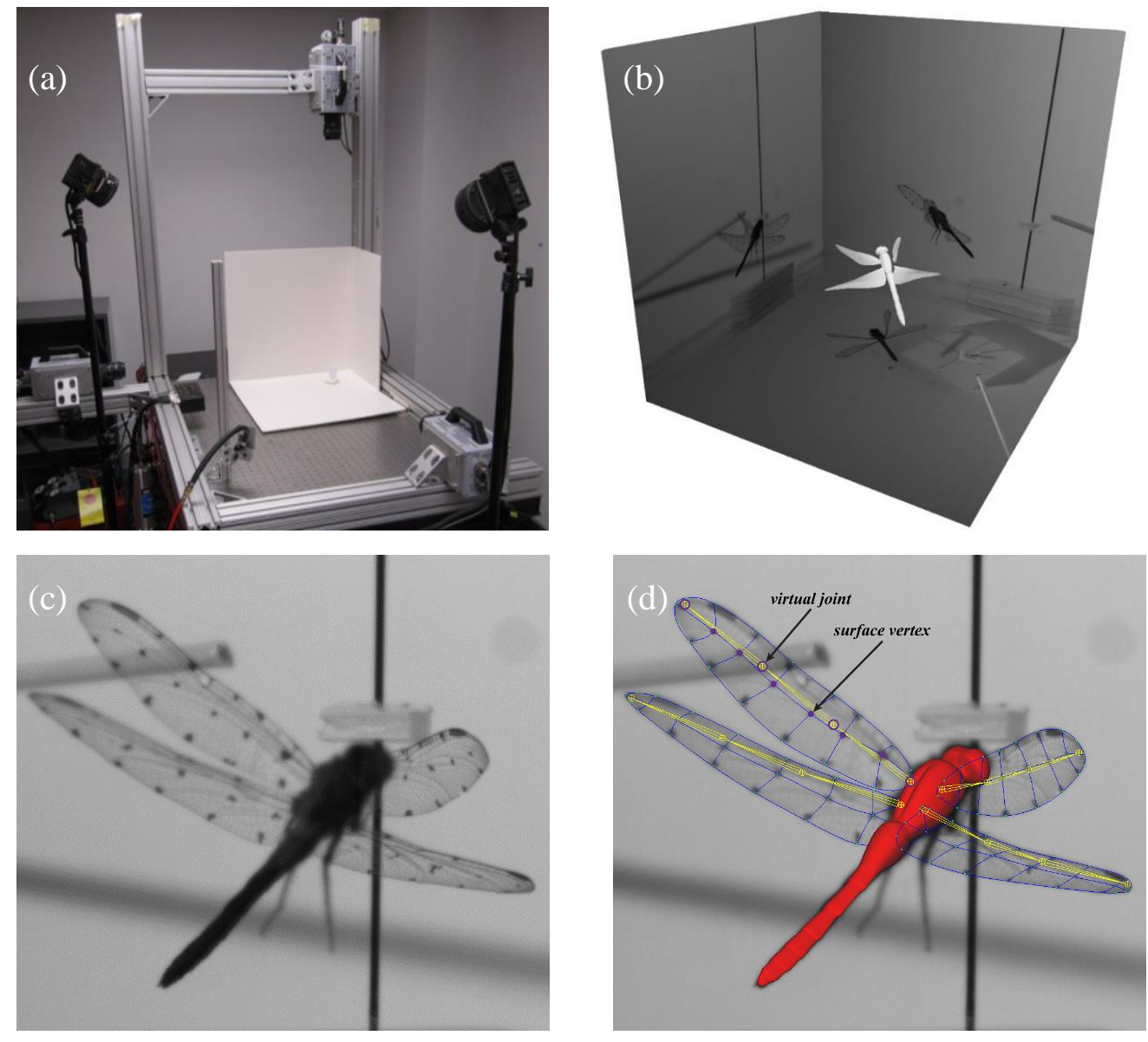

Figure 2-4. (a) Experimental setup; (b) reconstructed model based on three orthogonal projected high-speed images; (c) raw image of a hovering dragonfly; (d) schematic plots showing virtual joints configuration of the dragonfly wings.

The accuracy of the current method is also examined by comparing the kinematics and deformation measured based on both methods for the same case. Figure 2-5(a) shows the schematic plot of wing kinematics definitions, where $\phi, \theta$ and $\alpha$ stand for wing stroke 
angle, deviation angle and pitch angle, respectively. The corresponding time histories of those angles for right forewing of the hovering dragonfly are plotted in Figure 2-5(b). We can see that the results from both methods are similar, and the differences are less than $2 \%$. For wing deformation, we measure the wing camber to chord ratio $(\alpha)$ and twist angle $(\gamma)$ of right forewing of the hovering dragonfly and presented in Figure 2-5(c). The two deformation parameters are defined the same way that presented in Koehler's work [51]. We can see from Figure 2-5(c) that the results from joint-based reconstruction method are the same as the conventional method. The differences are less than $2 \%$.
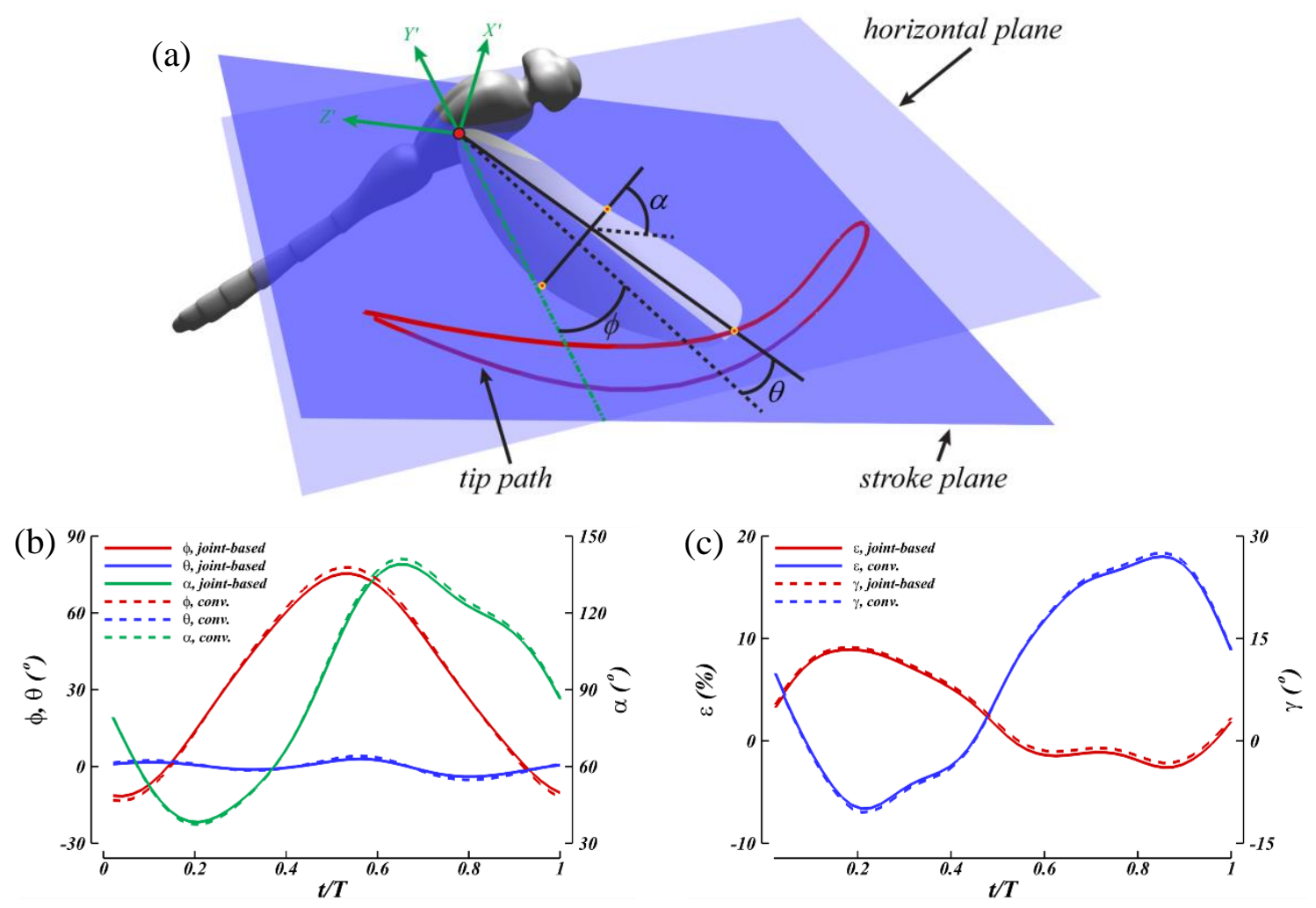

Figure 2-5. (a) Schematic plot of wing kinematics parameters definition; (b) time course of forewing kinematics measurements from the joint-based and the conventional method; (c) time course of forewing deformation measurements from the joint-based and the conventional method. The deformation parameters are measured at mid chord of the wing. 


\subsection{Spherical-coordinates-based Singular Value Decomposition (SSVD)}

SVD (also known as POD or PCA in some fields of application) is a powerful method for data analysis aimed at obtaining low-dimensional approximate descriptions of a highdimensional process or dataset [77], which is ddifferent from the low dimensional analysis of mode shapes in structural engineering. The most remarkable feature of the SVD is its optimality: it provides the most efficient way of capturing the dominant components of any dataset with only a finite and often surprisingly few numbers of modes. In gait analysis, PCA has yielded insights into human walking strategies and the interrelationships in terms of temporal, kinematic and kinetic variables. Urtasun and colleagues [80] have used PCA to identify invariant or common features within the whole body kinematics of a contemporary dance movement pattern. For animals in nature, Bozkurttas and colleagues [76] have used SVD to study the pectoral fin kinematics and its associated aerodynamics of bluegill sunfish. Representing the motions as a linear sum of principal components has become a widely accepted animation technique $[83,84]$. SVD and other similar methods are closely related, and the close connections and equivalence of these various methods can be found elsewhere [77].

Similar to the conventional SVD method [76], we have developed sphericalcoordinates-based SVD (SSVD) in this work. The spherical coordinate system is applied to describe the flapping wing motions in the current work, instead of using the conventional Cartesian coordinate system. The nature of flapping flight is that the wings are rotating about the wing roots, and also, each wing chord is rotating about the span axis. The spherical coordinate system can best fit the characteristics of flapping wing motions. In this section, we will introduce the detailed procedure of SSVD method. 


\subsubsection{Coordinate Transformation}

In this work, we focus on the decomposition of wing motions. A coordinate transformation is performed at first to remove the body translations, as well as the rotations, especially for flight with complex body motions like maneuvers. Body translations and Euler angles $\left(\psi_{B}, \theta_{B}\right.$ and $\phi_{B}$, which are body yaw, pitch and roll angle, respectively) are measured first. The following rotation matrix is then applied to remove the body motion for each snapshot:

$$
\left[\begin{array}{ccc}
\cos \psi_{b} & -\sin \psi_{b} & 0 \\
\sin \psi_{b} & \cos \psi_{b} & 0 \\
0 & 0 & 1
\end{array}\right]\left[\begin{array}{ccc}
\cos \theta_{b} & 0 & \sin \theta_{b} \\
0 & 1 & 0 \\
-\sin \theta_{b} & 0 & \cos \theta_{b}
\end{array}\right]\left[\begin{array}{ccc}
1 & 0 & 0 \\
0 & \cos \phi_{b} & -\sin \phi_{b} \\
0 & \sin \phi_{b} & \cos \phi_{b}
\end{array}\right]\left[\begin{array}{c}
X-X_{O} \\
Y-Y_{O} \\
Z-Z_{O}
\end{array}\right]=\left[\begin{array}{c}
X^{\prime} \\
Y^{\prime} \\
Z^{\prime}
\end{array}\right]
$$

where $\left(X_{O}, Y_{O}, Z_{O}\right)$ represents the body center location. $(X, Y, Z)$ and $\left(X^{\prime}, Y^{\prime}, Z^{\prime}\right)$ represent the motion in global and body local coordinate systems, respectively.

Figure 2-6 shows reconstructed flight motions of a takeoff maneuver dragonfly in global and body local coordinate systems. Wing tip trajectories are shown to emphasize the motions of wings in both cases. We can see that the wing motion in Figure 2-6(a) is greatly influenced by the body translations and rotations, and it may affect the decomposition results. After the body motion is removed, as we can see in Figure 2-6(b), the wing motions are reserved, and the wings are rotating about the wing joints. The spherical coordinate system can best fit the characteristics of the flapping wing motions in body local coordinate system. 

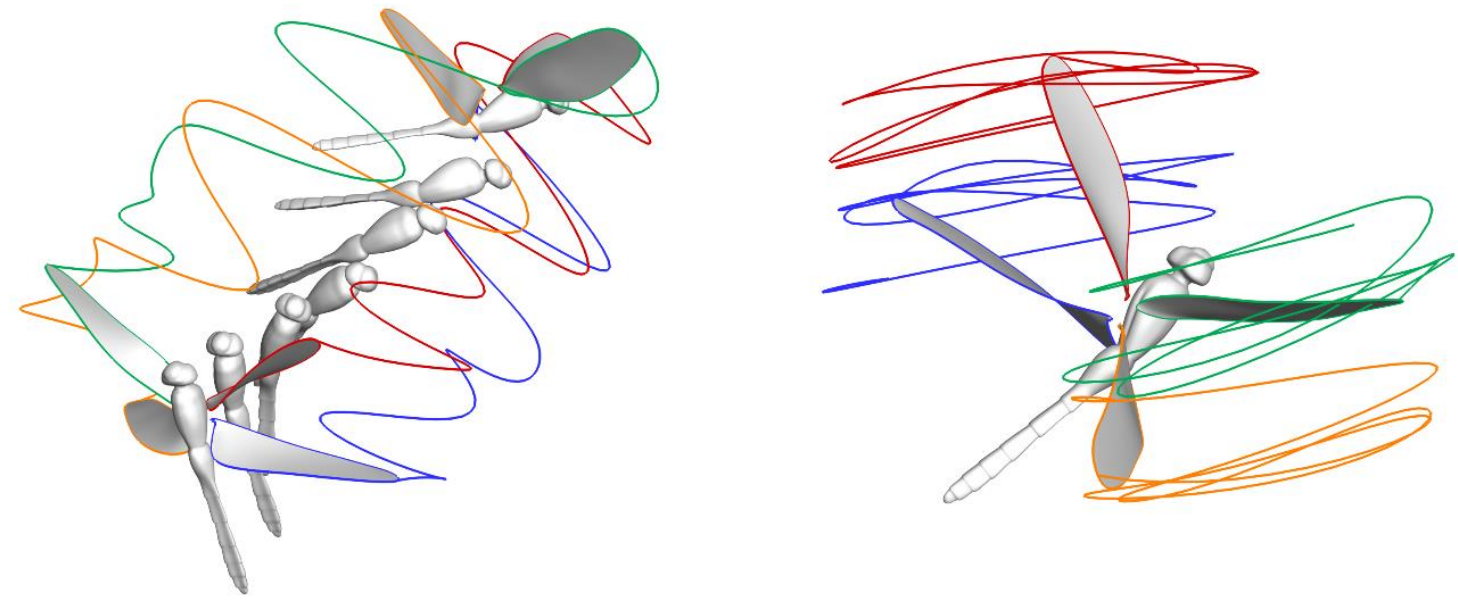

Figure 2-6. Reconstructed model of a takeoff maneuver dragonfly in global (a) and local (b) coordinate system. Colored curves are wing tip trajectories.

\subsubsection{Reference Snapshot Determination}

In order to perform a valid decomposition that can effectively extract wing deformation, a reference snapshot should be determined before the decomposition takes place. In this work, the snapshot with least wing deformation is chosen. The least square plane of wing shape, which can be mathematically described as a normalized plane equation $A x+B y+C z+D=0$, is determined at each time step. The coefficients $(A, B, C, D)$ are calculated via minimizing the standard deviation $Q$ from the following equation:

$$
Q=\sum_{i=1}^{m} R_{i}^{2}
$$

Where $R_{i}$ is the distance from the $i^{\text {th }}$ vertex on the wing surface to the least square plane, and it is defined as: 


$$
R_{i}=A x_{i}+B y_{i}+C z_{i}-D
$$

The reference snapshot is then chosen as the one with smallest standard deviation $Q$ among all time steps.

\subsubsection{Displacement Matrix and Singular Value Decomposition}

SVD can be considered as an extension of the traditional eigenvalue decomposition for the non-square matrix, which contains a dataset that represents the wing motion in both time and space. Displacements of all $m$ nodes on the wing surface at $n$ distinct instants in time are stored in this matrix, named displacement matrix. The displacement matrix (denoted by $\mathbf{A}$ ) is as follows:

$$
\mathbf{A}=\left[\begin{array}{cccc}
\Delta r_{1}^{1} & \Delta r_{1}^{2} & \cdots & \Delta r_{1}^{n} \\
\Delta \theta_{1}^{1} & \Delta \theta_{1}^{2} & \cdots & \Delta \theta_{1}^{n} \\
\Delta \phi_{1}^{1} & \Delta \phi_{1}^{2} & \cdots & \Delta \phi_{1}^{n} \\
\vdots & \vdots & \vdots & \vdots \\
\Delta r_{m}^{1} & \Delta r_{m}^{2} & \cdots & \Delta r_{m}^{n} \\
\Delta \theta_{m}^{1} & \Delta \theta_{m}^{2} & \cdots & \Delta \theta_{m}^{n} \\
\Delta \phi_{m}^{1} & \Delta \phi_{m}^{2} & \cdots & \Delta \phi_{m}^{n}
\end{array}\right]_{3 m \times n}
$$

The displacements stored in above matrix are calculated as follows:

$$
\left\{\begin{array}{l}
\Delta r_{i}^{t}=r_{i}^{t}-r_{i}^{r e f} \\
\Delta \theta_{i}^{t}=\theta_{i}^{t}-\theta_{i}^{r e f} \\
\Delta \phi_{i}^{t}=\phi_{i}^{t}-\phi_{i}^{r e f}
\end{array}\right.
$$


where $\left(r_{s}^{t}, \theta_{s}^{t}, \phi_{s}^{t}\right)$ denote the coordinates of the node $s$ at time instant $t$. Note that the spherical coordinate system is used here. $\left(r_{i}^{r e f}, \theta_{i}^{r e f}, \phi_{i}^{r e f}\right)$ denote the coordinates of the node $i$ at a specific reference time instant. An SVD of the displacement matrix $\mathbf{A}$ can then be factorized as

$$
\mathbf{A}_{3 m \times n}=\mathbf{U}_{3 m \times 3 m} \mathbf{S}_{3 m \times n} \mathbf{V}_{n \times n}^{T}
$$

where $\mathbf{U}_{3 m \times 3 m}$ and $\mathbf{V}_{n \times n}^{T}$ are two orthogonal unitary matrices; $\mathbf{S}_{3 m \times n}$ is a diagonal matrix in which the diagonal values are called the singular values of $\mathbf{A}$, which are unique. The diagonal elements $\mathbf{S}_{i i}$ consist of $r=\min (m, n)$ non-negative numbers $\lambda_{i}$, which are arranged in descending order, i.e. $\lambda_{1} \geq \lambda_{2} \geq \cdots \geq \lambda_{n} \geq 0$. Within the SSVD procedure, the $\lambda_{i}$ values are the square roots of the eigenvalues of $\mathbf{A} \mathbf{A}^{T}$, whereas the eigenvectors of $\mathbf{A} \mathbf{A}^{T}$ make up the columns of $\mathbf{U}$ and $\mathbf{V}^{T}$ respectively. In the above expression, $\mathbf{V}$ represents the change in each mode with time, and $\mathbf{U}$ contains the eigenvectors corresponding to the spatial distribution of the modes. The singular values $\lambda_{i}$ can be interpreted as the weight contributions of each mode in the SSVD. Thus, the 'shape' of any particular mode (say the $\mathrm{k}^{\text {th }}$ mode) can be extracted by zeroing out all the singular values except for the $\mathrm{k}^{\text {th }}$ value, and reconstructing from the SSVD as in Eqn(7). Similarly, lower dimensional (say rank $K<n)$ approximations to the dataset can be obtained by using an approximation to $\mathbf{S}$ denoted by $\mathbf{S}_{K}$ wherein $\lambda_{K+1}=\lambda_{K+2}=\cdots=\lambda_{n}=0$ and reconstructing from the SSVD as follows:

$$
\mathbf{A}_{K}=\mathbf{U S}_{K} \mathbf{V}^{T}
$$


The displacement matrix $\mathbf{A}$ is now subjected to SSVD. In the following part, the forewing motion of a hovering dragonfly will be used to demonstrate the decomposition results. 30 snapshots of the wing motion for one complete flapping cycle are used in the decomposition. As expected, the SSVD leads to 30 distinct singular values, and the spectrum for the first ten singular values of the wing motion is shown in Figure 2-7 along with a cumulative plot for the same data. The normalized singular value for $\mathrm{k}^{\text {th }}$ mode $\lambda_{k}^{*}$ is defined as:

$$
\lambda_{k}^{*}=\lambda_{k} / \sum_{i=1}^{n} \lambda_{i}
$$

The singular values are normalized by the sum of all singular values. Therefore, the cumulative values sum to unity. A number of interesting observations can be made from this plot. First, the singular value spectrum shows three distinct ranges: the first between mode $1-2$, in which we see a rapid decrease in the amplitude, the second from mode 2 - 4 in which there is a much slower reduction in amplitude and, finally, the range from mode 4 - 30 that has negligible (less than $2 \%$ ) total contribution. The rapid initial decrease in the spectrum is significant which suggests that a small number of modes contain most of the essential features of the wing gait. In fact, the cumulative values show that the first two and three modes capture about $93.1 \%$ and $96.0 \%$ respectively of the total motion. In fact, only the first mode captures close to $84.7 \%$ of the motion of the wing, which is a clear demonstration of the ability of SSVD to represent the dataset with the least possible number of modes. 


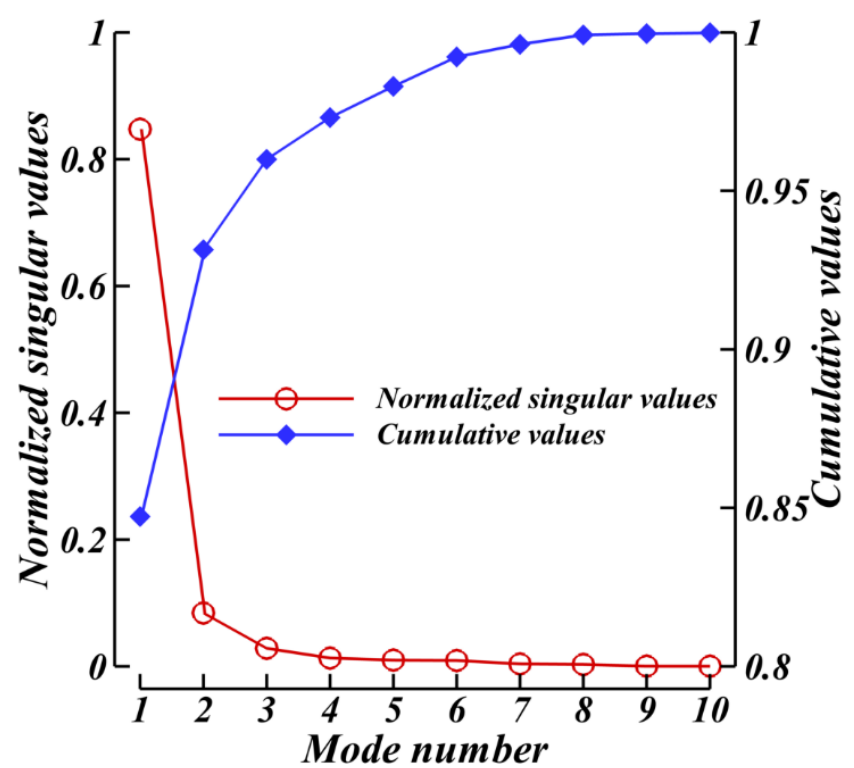

Figure 2-7. The SSVD spectrum of the first ten modes for the dragonfly's right forewing kinematics. The left ordinate shows $\left|\lambda_{i}\right| / \sum\left|\lambda_{i}\right|$, and the right ordinate shows the cumulative value of the left ordinate.

The gait corresponding to individual modes can be extracted as described above, and the surface conformations for each of these extracted modes are then constructed using the original wing mesh with triangular elements. The first two modes are highly distinct and relatively easy to interpret, and we briefly describe the key qualitative features of these modes. Figure 2-8(b, c) shows mode 1 and mode 2 at five different times during one flapping cycle. Also shown on the left for direct comparison are the wing motion from the experiment (also called the 'mode-all' case, since it contains all the SSVD modes). In these figures, the colors reflect wing deformation by plotting contours of distances between vertices on the wing surface to the corresponding least square plane of the wing. Mode 1 involves very large rotating motion about the wing root, which is called the 'flapping' 
motion. The wing flaps back and forth with certain offset angle. And also, the mode one shows minimum deformation during the stroke. This mode is actively produced by the dragonfly through flight mussels at the wing root.
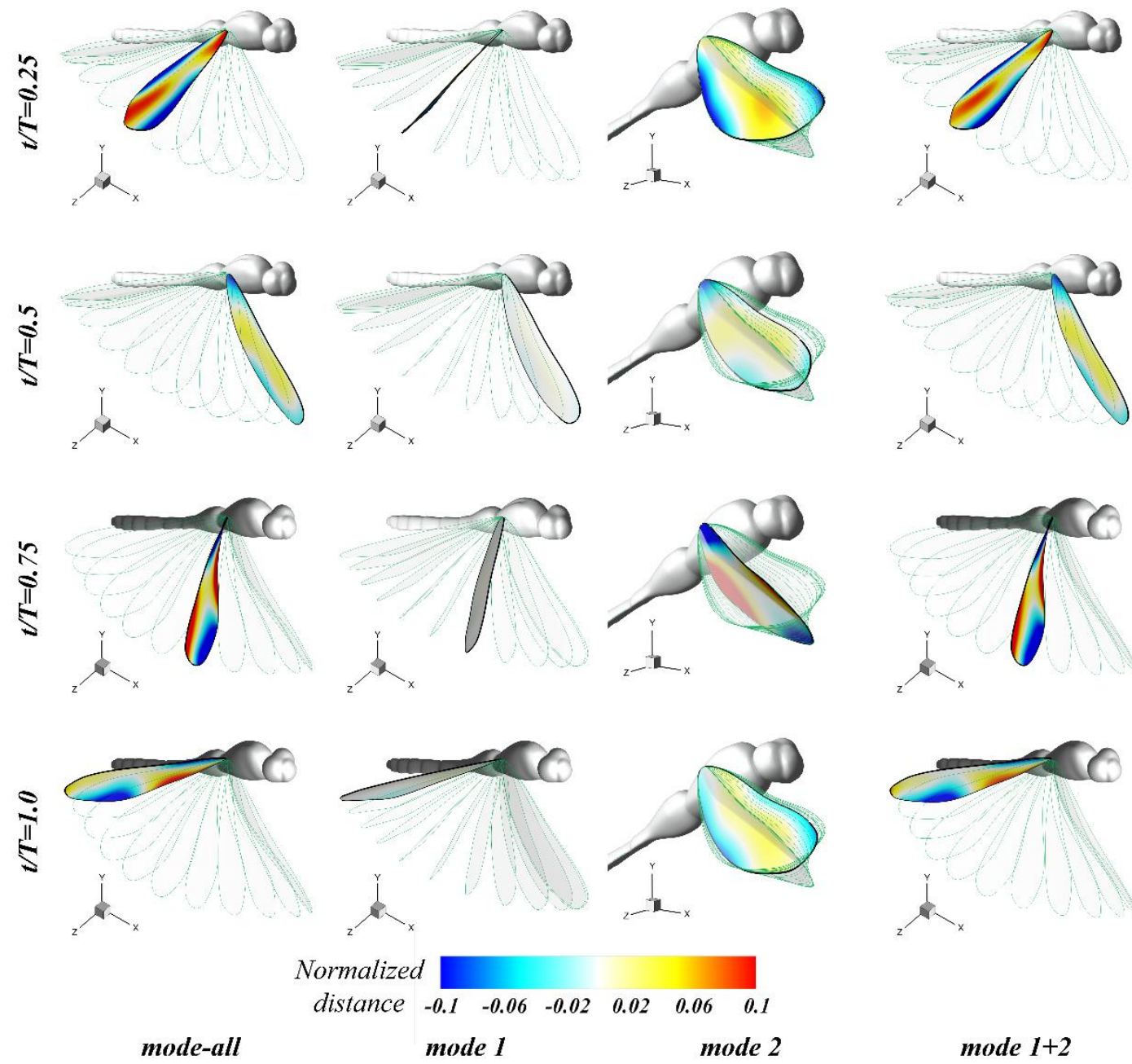

Normalized

distance

(a) mode 1

(b)

mode 2

(c)

mode $1+2$

(d)

Figure 2-8. Wing motions of (a) experimental kinematics (also called the mode-all case), (b) SSVD mode 1, (c) mode 2 and (d) low-dimensional model mode 1+2. The wings are colored with distances between wing surfaces and corresponding least square planes. The distances are normalized by wing mid chord length. 
Mode 2 is a twisted motion primarily in the span-wise direction, which occurs along the span axis of the wing. It presents as chord-wise rotations of the wing during the reversal phase. In contrast to mode 1, this mode is primarily a result of flow-induced deformation. It can be deduced from the fact that there are no muscles in the wing surface that could produce deformation in the wing. Furthermore, the deformation is primarily in the direction of the flow relative to the wing motion, which supports the assertion that this mode is flowinduced. The rest of the modes in the spectrum are associated with relatively small motions that are not very distinct. We, therefore, do not describe these individually, although we will consider the effect of mode 3 on the aerodynamics in the following sections.

\subsubsection{Low-dimensional Models}

SSVD has decomposed the wing motion into its orthogonal components and helped us understand the key features of the nature flyers' wing motion. The SSVD results can also be used to reconstruct low-dimensional approximations of the mode-all case using a subset of the orthogonal modes. Lower dimensional models of the wing gait are synthesized by successively adding modes to Mode 1 . The forewing motion of a hovering dragonfly is used to demonstrate the method. Figure 2-8(a, b, d) shows the surface snapshots at four different time instances during one flapping cycle for mode 1 and mode $1+2$ in comparison to the complete (mode-all) motion. The similarity between the wing shapes for Mode $1+2$ and the Mode-All/experiment cases is evident in this figure. Removal of higher SSVD modes from the motion is analogous to filtering the experimental data in space and time. 


\subsubsection{Convergence Studies and Motion Percentage}

As mentioned before, we are using 30 snapshots in one flapping cycle to perform the decomposition and construct low-dimensional models in the case discussed above. Here lies a question of whether or not the snapshot size will affect the decomposition results. In order to answer the question, convergence studies of the SSVD method are conducted. We have used various snapshot sizes (10 - 60 snapshots) to perform the decomposition and build low-dimensional models (mode $1+2$ ) to evaluate the results. To best demonstrate the results, we define motion percentages $\varepsilon$ directly from the motions of low-dimensional models. Motion percentage $\varepsilon$ at a certain time step for each snapshot size is calculated based on the following equation:

$$
\varepsilon=\frac{\sum_{i=1}^{m} \sqrt{\left(X_{i}^{\text {low }}-X_{i}^{\text {ref }}\right)^{2}+\left(Y_{i}^{\text {low }}-Y_{i}^{\text {ref }}\right)^{2}+\left(Z_{i}^{\text {low }}-Z_{i}^{\text {ref }}\right)^{2}}}{\sum_{i=1}^{m} \sqrt{\left(X_{i}^{\text {all }}-X_{i}^{\text {ref }}\right)^{2}+\left(Y_{i}^{\text {all }}-Y_{i}^{\text {ref }}\right)^{2}+\left(Z_{i}^{\text {all }}-Z_{i}^{\text {ref }}\right)^{2}}}
$$

Where $(X, Y, Z)$ stands for the coordinates of the vertex on the wing surface; the superscript 'low', 'ref' and 'all' represent vertices of low-dimensional model, reference frame, and mode-all model, respectively; the subscript ' $i$ ' denotes the $i^{\text {th }}$ vertex. Time histories of $\varepsilon$ for each snapshot size are plotted in Figure 2-9. We can see that for lowdimensional models with larger snapshot size (30,45 and 60), the motion percentage $\varepsilon$ is larger and converge to a certain value. For smaller sizes (10, 15 and 25), the curves are very different. Table 2-3 lists averaged motion percentages $\bar{\varepsilon}$ and normalized singular values $\lambda^{*}$ of mode $1+2$ for various snapshot sizes. We can see that $\bar{\varepsilon}$ values converge while the snapshot sizes are larger than 30. Differently, the normalized singular values $\lambda^{*}$, 
which are used to quantify the weight of low-dimensional models in previous sections, stay the same (around 93\%) for all snapshot sizes. This is because $\lambda^{*}$ are calculated based on the mode-all motion of each snapshot size, while the references of averaged motion percentages $\bar{\varepsilon}$ are all from the largest snapshot size. The averaged motion percentage $\bar{\varepsilon}$ is more proper to describe the weight of low-dimensional modes, especially for small snapshot sizes (below 30 snapshots in one complete flapping cycle). However, for larger snapshot sizes greater than 30, both methods are fine. From the convergence studies above, we choose the snapshot size as 30 in the following discussions.

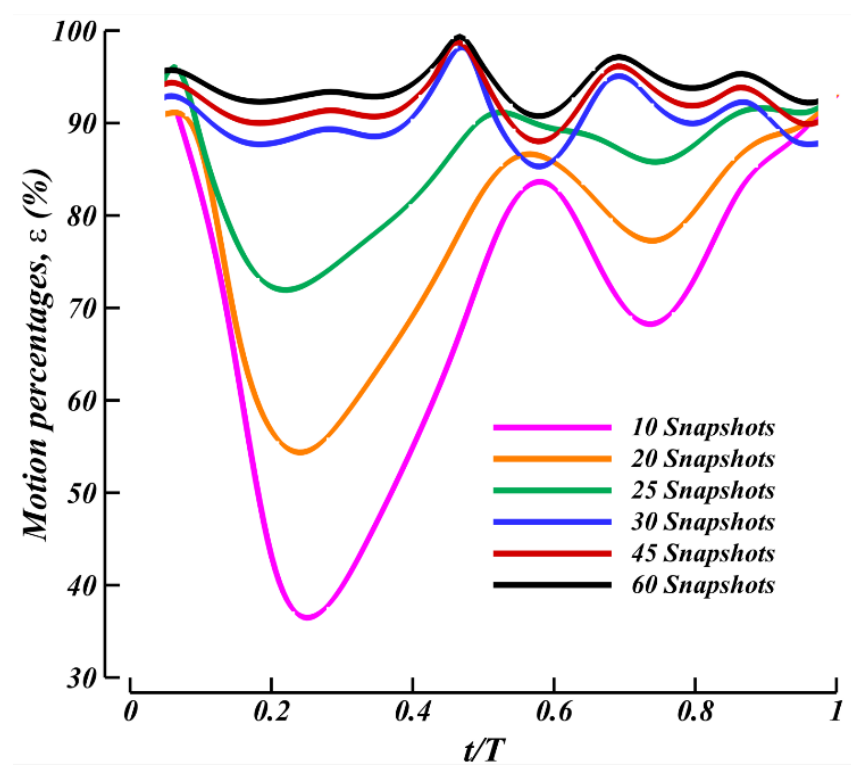

Figure 2-9. Motion percentage histories of low-dimensional models (mode $1+2$ ) for different snapshot sizes. 
Table 2-3. Averaged motion percentages $\bar{\varepsilon}$ and normalized singular values $\lambda^{*}$ of lowdimensional models (mode 1+2) for different snapshot sizes.

\begin{tabular}{lcc}
\hline \hline Snapshots Sizes & $\bar{\varepsilon}$ & $\lambda^{*}$ \\
\hline 10 & $68.5 \%$ & $93.3 \%$ \\
20 & $77.0 \%$ & $93.2 \%$ \\
25 & $85.4 \%$ & $93.2 \%$ \\
30 & $90.4 \%$ & $93.1 \%$ \\
45 & $92.3 \%$ & $93.1 \%$ \\
60 & $94.1 \%$ & $93.1 \%$ \\
\hline
\end{tabular}

\subsubsection{SSVD Analysis of Various Flapping Propulsors}

In the current study, we have conducted SSVD analysis on the flapping motion of various propulsors in nature. It includes the wing motions of dragonfly, damselfly, cicada and hummingbird. And also in different flight modes, including hovering, cruising, and maneuvering. The decomposition results show that all cases share similar SSVD modes that we discussed previously (mode 1: flapping mode, mode 2: deformation mode). As shown in Table 2-4, both $\bar{\varepsilon}$ and $\lambda^{*}$ values are listed, and they show similar trends among all cases. Here we just use $\lambda^{*}$ to describe the results. The $\lambda^{*}$ values of mode $1+2$ range from $86.3 \%$ to $93.7 \%$. Most of the motions are captured by low-dimensional models SSVD mode $1+2$. The $\lambda^{*}$ values of mode 1 range from $78.1 \%$ to $86 \%$ while that of mode 2 range from $7.7 \%$ to $15.2 \%$. Those values suggest different characteristics of those flapping motion.

For smaller aspect ratio wings, such as the wings of hummingbird and cicada (we consider the forewing and hindwing of cicada as one single wing since they are hooked 
with each other during the flapping motion), the $\lambda^{*}$ values for mode 2 are larger. It suggests that they present more deformation during the flight. On the other hand, smaller $\lambda^{*}$ values correlate to large aspect ratio wings, like the wings of damselfly. The wing deformation is relatively small in such wings. For simpler motions, such as hovering and cruising, the $\lambda^{*}$ values of mode $1+2$ are higher than that of complex motions, like maneuvering. This indicates that higher SSVD modes (mode 3, 4, etc...) contribute more to complex motions. Moreover, the mode 2 has a larger contribution to the maneuvering flights and smaller contribution in cruising flights, which suggests that the wing deformation plays a more significant role in maneuvering flights when compared to cruising flights.

Table 2-4. SSVD analysis of several flapping propulsors in nature.

\begin{tabular}{|c|c|c|c|c|c|c|c|c|c|}
\hline \multirow{2}{*}{ Species } & \multirow{2}{*}{ Motion } & \multirow{2}{*}{ Wings } & \multirow{2}{*}{ AR } & \multicolumn{3}{|c|}{$\bar{\varepsilon}(\%)$} & \multicolumn{3}{|c|}{$\lambda^{*}(\%)$} \\
\hline & & & & M1 & M2 & M12 & M1 & M2 & M12 \\
\hline \multirow{8}{*}{ dragonfly } & \multirow{2}{*}{ hover } & fore & 5.0 & 82.3 & 8.1 & 90.4 & 84.7 & 8.4 & 93.1 \\
\hline & & hind & 4.3 & 81.0 & 9.2 & 90.2 & 83.3 & 9.6 & 92.9 \\
\hline & \multirow{2}{*}{ cruise } & fore & 5.0 & 84.1 & 7.5 & 91.6 & 86.0 & 7.7 & 93.7 \\
\hline & & hind & 4.2 & 83.2 & 8.8 & 92.0 & 85.1 & 9.0 & 94.1 \\
\hline & \multirow{4}{*}{ maneuver } & inner fore & \multirow{2}{*}{5.1} & 78.8 & 9.9 & 88.7 & 81.1 & 10.2 & 91.3 \\
\hline & & outer fore & & 78.5 & 9.7 & 88.2 & 80.6 & 9.8 & 90.4 \\
\hline & & inner hind & \multirow{2}{*}{4.2} & 77.8 & 10.8 & 88.6 & 80.4 & 11.3 & 91.7 \\
\hline & & outer hind & & 77.6 & 10.5 & 88.1 & 80.6 & 10.8 & 91.4 \\
\hline cicada & cruise & right & 2.5 & 76.5 & 14.2 & 90.7 & 78.1 & 15.2 & 93.3 \\
\hline \multirow{6}{*}{ damselfly } & \multirow{2}{*}{ cruise } & fore & 6.6 & 83.2 & 6.6 & 89.8 & 84.9 & 8.2 & 93.1 \\
\hline & & hind & 6.2 & 82.5 & 6.8 & 89.3 & 84.2 & 8.4 & 92.6 \\
\hline & \multirow{4}{*}{ maneuver } & inner fore & \multirow{2}{*}{6.6} & 79.2 & 7.0 & 86.2 & 80.9 & 8.5 & 89.4 \\
\hline & & outer fore & & 78.6 & 7.5 & 86.1 & 80.3 & 9.0 & 89.3 \\
\hline & & inner hind & \multirow{2}{*}{6.2} & 79.1 & 7.3 & 86.4 & 80.9 & 8.5 & 89.4 \\
\hline & & outer hind & & 78.3 & 7.9 & 86.2 & 80.0 & 9.2 & 89.2 \\
\hline \multirow{3}{*}{ hummingbird } & hover & right & 4.0 & 76.6 & 8.7 & 85.3 & 77.8 & 10.2 & 88.0 \\
\hline & \multirow{2}{*}{ maneuver } & inner & \multirow{2}{*}{4.0} & 73.9 & 8.8 & 82.7 & 75.8 & 10.5 & 86.3 \\
\hline & & outer & & 74.5 & 8.6 & 83.1 & 76.6 & 10.4 & 87.0 \\
\hline
\end{tabular}


The SSVD analysis suggests one natural approach to the development of the robotic propulsors. Since a small number of modes capture a significant portion of the motion, it stands to reason that a systematic procedure for developing a robotic propulsor would involve designing actuation mechanisms that reproduce a small number of these modes. The question that remains to be answered is what kind of performance can we expect from these lower dimensional wing models, and how does the performance scale as we include additional modes? This will allow us to make a rational compromise between the complexity of propulsor design and propulsor performance. It should be noted here that the performance is a consequence of the flow associated with these low-dimensional models. Thus, even though the modes are kinematically linear (and therefore additive), the performances are not expected to scale linearly with the modes since the flow is governed by the Navier-Stokes equations which are nonlinear. Thus, the answer to the above question requires that we explicitly determine the performances for these low-dimensional models.

\subsection{Immersed Boundary Method for Deformable Attaching Bodies (IBM-DAB)}

All natural propulsors are attached to animal bodies. Those propulsors are usually thin and present large deformation during the flapping motion. Zero thickness deformable membranes are usually used to model those propulsors in computational studies $[46,85$, 86]. The animal body is not included or treated as separate part from the propulsors (propulsors are not firmly attached to the body) in those computational studies due to the focus of the works or the computational difficulties regarding the attachment of the propulsors to the animal body. However, recent studies show that the body of an animal is 
an important part of the flapping propulsion system, and the distance between the body and propulsor is a key control parameter of the entire performance of the propulsion system [87, 88]. In the current work, an immersed boundary method for deformable attaching bodies (IBM-DAB) is developed to handle the simulations of solid bodies with deformable attaching membrane bodies.

\subsubsection{Governing Equations and Discretization Scheme}

The governing equations considered are the 3D unsteady Navier-Stokes equations for a viscous incompressible flow with constant properties given by:

$$
\left\{\begin{array}{l}
\frac{\partial u_{i}}{\partial x_{i}}=0 \\
\frac{\partial u_{i}}{\partial t}+\frac{\partial\left(u_{i} u_{j}\right)}{\partial x_{j}}=-\frac{1}{\rho} \frac{\partial u_{i}}{\partial t}+v \frac{\partial}{\partial x_{j}}\left(\frac{\partial u_{i}}{\partial x_{j}}\right)
\end{array}\right.
$$

Where $i, j=1,2,3, u_{i}$ are the velocity components, $p$ is the pressure, and $\rho$ and $v$ are the fluid and kinematic viscosity.

The Navier-Stokes equation(10) are discretized using a cell-centered, collocated (nonstaggered) arrangement of the primitive variables $u_{i}$ and $p$. In addition to the cell-center velocities $u_{i}$, the face-center velocities $U_{i}$, are computed (see Figure 2-10). The equations are integrated in time using the fractional step method, which consists of three sub-steps. In the first sub-step of this method, a modified momentum equation is solved and an intermediate velocity $u^{*}$ is obtained. A second-order, Adams-Bashforth scheme is employed for the convective terms while the diffusion terms are discretized using an 
implicit Crank-Nicolson scheme, which eliminates the viscous stability constraint. In this sub-step, the following modified momentum equation is solved at the cell-nodes:

$$
\frac{u_{i}^{*}-u_{i}^{n}}{\Delta t}=\frac{1}{2}\left[3 \frac{\delta\left(U_{j}^{n} u_{i}^{n}\right)}{\delta x_{j}}-\frac{\delta\left(U_{j}^{n-1} u_{i}^{n-1}\right)}{\delta x_{j}}\right]=-\frac{1}{\rho} \frac{\delta p^{n}}{\delta x_{i}}+\frac{1}{2} v\left[\frac{\delta}{\delta x_{j}}\left(\frac{\delta u_{i}^{*}}{\delta x_{j}}\right)+\frac{\delta}{\delta x_{j}}\left(\frac{\delta u_{i}^{n}}{\delta x_{j}}\right)\right]
$$

Where $\frac{\delta}{\delta x}$ corresponds to a second-order central difference. This equation is solved using a line-SOR scheme [89].

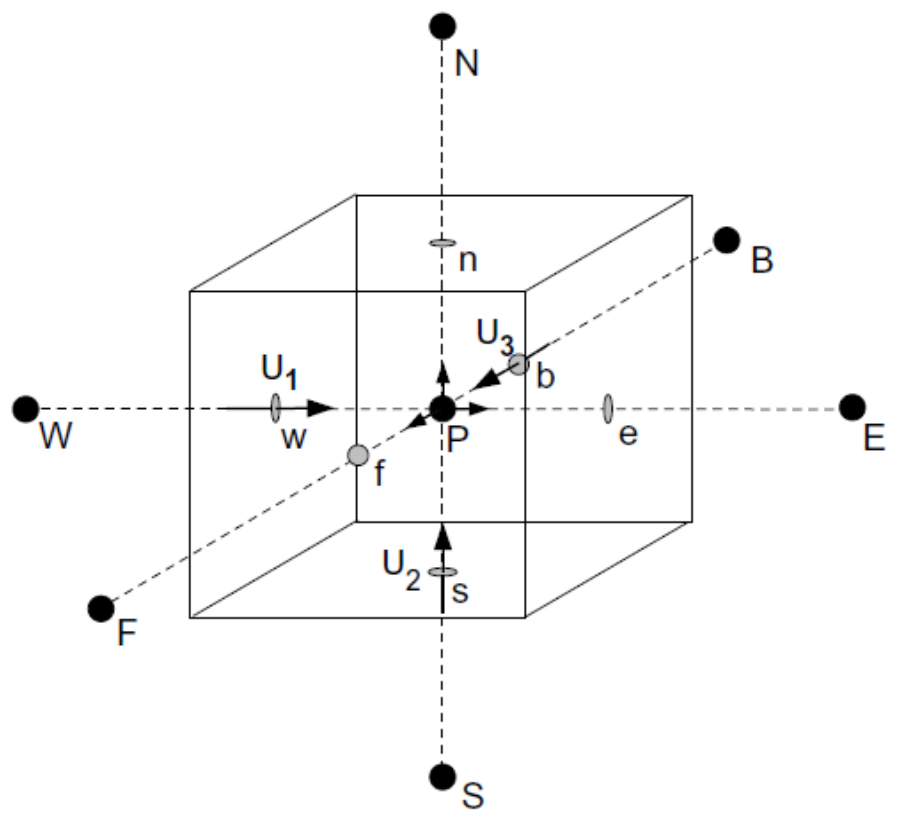

Figure 2-10. Schematic describing the naming convention and location of velocity components employed in the spatial discretization of the governing equations.

The second sub-step requires the solution of the pressure correction equation: 


$$
\frac{u_{i}^{n+1}-u_{i}^{*}}{\Delta t}=-\frac{1}{\rho} \frac{\delta p^{\prime}}{\delta x_{i}}
$$

which is solved with the constraint that the final velocity $u_{i}^{n+1}$ be divergence-free. This gives the following Poisson equation for the pressure correction:

$$
\frac{1}{\rho} \frac{\delta}{\delta x_{i}}\left(\frac{\delta p^{\prime}}{\delta x_{i}}\right)=\frac{1}{\Delta t} \frac{\delta U_{i}^{*}}{\delta x_{i}}
$$

and a Neumann boundary condition imposed on the pressure correction at all boundaries.

This Poisson equation is solved with a highly efficient geometric multigrid method [90], which employs a modified strongly implicit procedure (MSIP) [91] smoother. The ability to employ such methods is another key advantage of the current Cartesian grid approach over body-conformal unstructured grid approaches. Geometrical multigrid methods are relatively simple to implement and have very limited memory overhead. Furthermore, when coupled with powerful smoothers like line-Gauss-Siedel or MSIP, they can lead to a numerical solution of the pressure Poisson equation which scales almost linearly with the number of grid points. In contrast, for unstructured body-conformal methods, one has to either resort to algebraic multigrid methods $[92,93]$ or other more complex methods such as agglomeration multigrid [94]. Another choice for solving the pressure Poisson equation would be Krylov subspace based methods (such as conjugate gradient or GMRES) but these require effective preconditioners to provide good performance. Our past experience with both stationary and non-stationary iterative methods [95-97] indicates that geometric multigrid methods are very well suited for sharp interface immersed boundary methods, and we have therefore used this method in the current solver. 
Once the pressure correction is obtained, the pressure and velocity are updated as:

$$
\left\{\begin{array}{l}
p^{n+1}=p^{n}+p^{\prime} \\
u_{i}^{n+1}=u_{i}^{*}-\Delta t \frac{1}{\rho}\left(\frac{\delta p^{\prime}}{\delta x_{i}}\right)
\end{array}\right.
$$

\subsubsection{Immersed Boundary and Cell Identification}

The motions of natural animals are reconstructed using the method introduced in Section 2.1, and the surface of a reconstructed model is represented as unstructured mesh with triangular elements. Figure 2-11(a) shows the surface mesh over a baby trout which is being used to examine the fluid dynamics of fishes' steady swimming. The trunk body of the fish is modeled as "solid body", and the fish fins are modeled as "membrane body", which presents zero thickness in the simulation.

The unstructured surface mesh is "immersed" into the Cartesian grid and Figure 2-11(b) shows this for the particular case of the baby trout. The next step is to develop all the computational machinery that is needed to implement the ghost-cell methodology for such immersed boundaries. We take the "solid body" as an example to demonstrate the method. We first identify cells whose nodes are inside the solid boundary (termed "solid cell") and cells that are outside the body (termed "fluid cell"). A conventional method [97] for this in a normal situation is shown in Figure 2-12(a). We deal with two cells ( $P_{1}$ and $P_{2}$ ) here. Two elements $\left(e_{1}\right.$ and $e_{2}$ ), which are closest to the corresponding cells, are found first. Vectors $v_{1}$ and $v_{2}$ are then formed by connecting the centers of the elements and the centers of the cells. The surface normal vectors of the two elements are marked as $n_{1}$ and 
$n_{2}$. The angle between $v_{1}$ and $n_{1}$ is $a_{1}$, and the angle between $v_{2}$ and $n_{2}$ is $a_{2}$. It is clear that the cell $P_{i}$ is a solid cell if the angle $a_{i}$ greater than $90^{\circ}$, otherwise, the cell is a fluid cell.

(a)

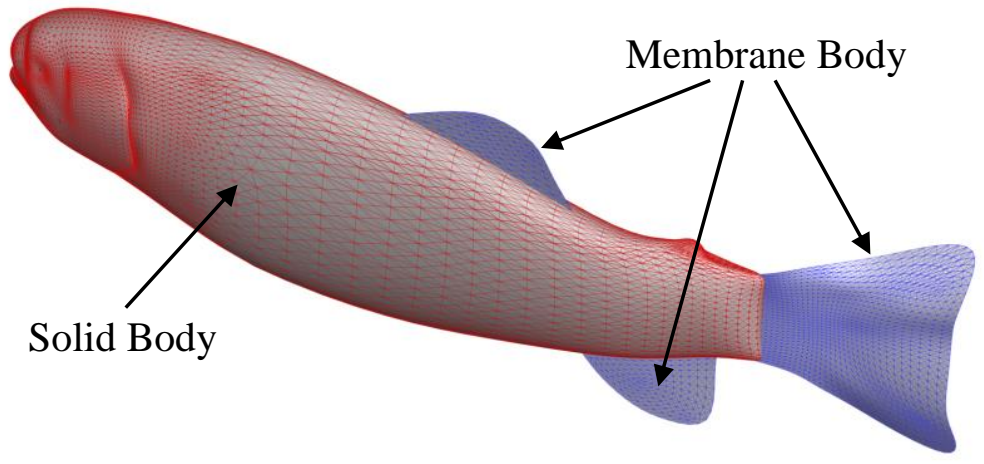

(b)

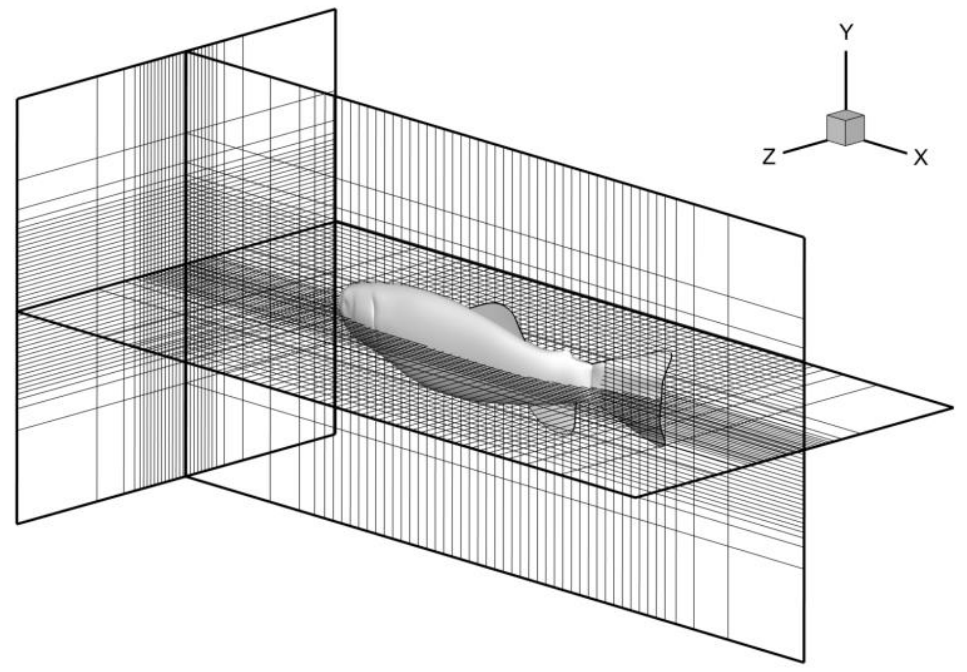

Figure 2-11. (a) Example of a baby trout model with unstructured surface mesh of triangular elements; (b) representative example showing the baby trout immersed in a nonuniform Cartesian grid. 
(a)

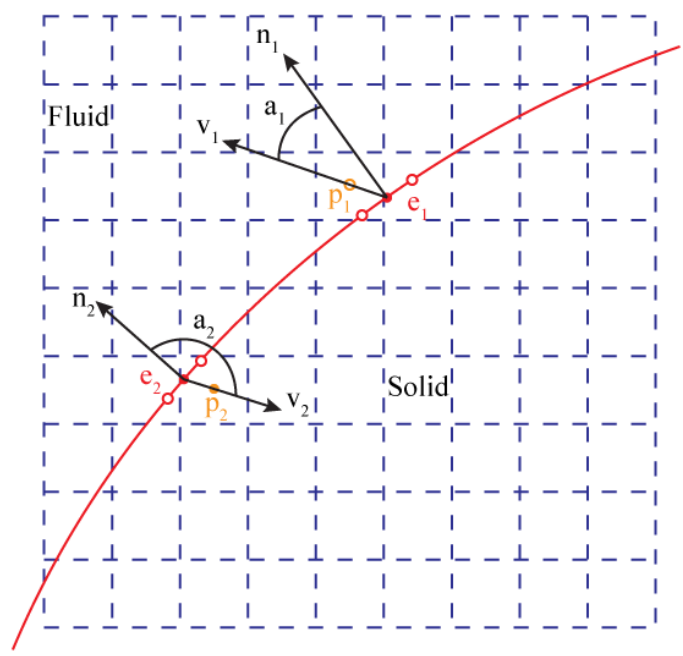

(c)

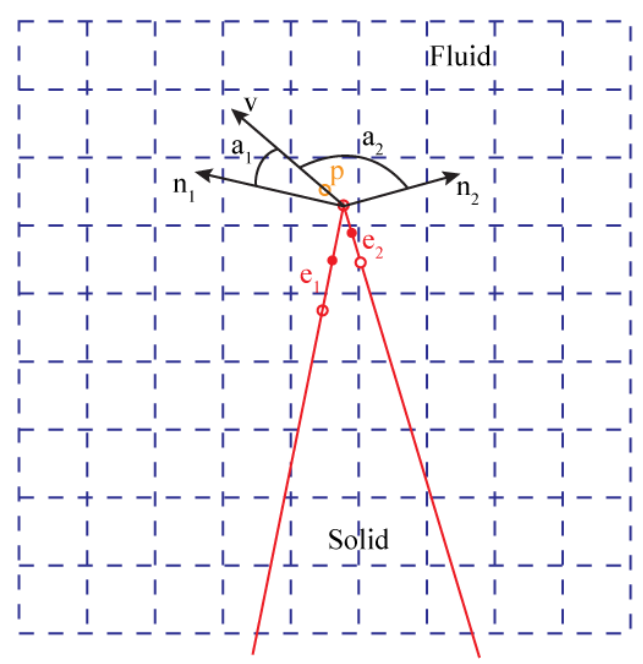

(b)

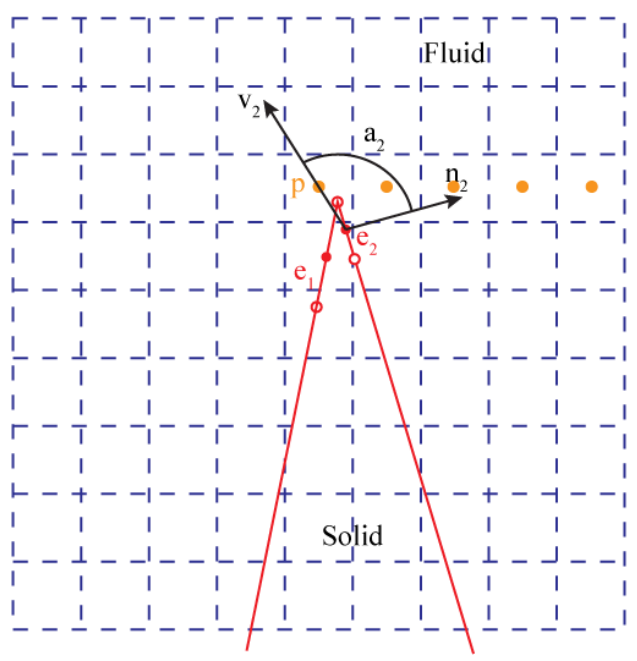

Immersed boundary

Boundary vertex

Boundary element center

Fluid cell

Solid cell

Figure 2-12. (a) Cell identification in a normal situation using the conventional method; (b) cell identification in a sharp edge situation using the conventional method; (c) cell identification in a sharp edge situation using the current method.

The conventional method works well in most of the situations. However, it makes mistakes when dealing with "sharp edges". As shown in Figure 2-12(b), cell $P$, which is 
a fluid cell, is subjected to the cell identification. Due to the different size of each element, the closest element to cell $P$ is found as $e_{2}$. Apparently, the angle $a_{2}$ is greater than $90^{\circ}$, and the cell is wrongly identified as a solid cell. A direct consequence of this is that all cells next to the cell $P$ are identified as solid cells due to a fast cell identification algorithm. Non-physical simulation results are obtained due to the mistakes correspond to cell identification.

A modified method is developed to solve the above problems. As shown in Figure 2-12(c), the new method is demonstrated in the same situation as in Figure 2-12(b). Instead of finding the closest element to the target cell, the closest vertex is found. In this case, the closed vertex is at the sharp tip of the immersed boundary. Vector $v$ is then formed by connecting the vertex and the center of the cell $P$. Next, the elements which share the vertex are found ( $e_{1}$ and $e_{2}$ ), and the corresponding surface normal vectors are $n_{1}$ and $n_{2}$. The angle between $v_{1}$ and $n_{1}$ is $a_{1}$, and the angle between $v_{2}$ and $n_{2}$ is $a_{2}$. Now, if the value $\min \left(a_{1}, a_{2}\right)$ is greater than $90^{\circ}$, the cell is identified as a solid cell, otherwise, it is a fluid cell. The cell $P$ in Figure 2-12(c) is successfully identified as a fluid cell with this new method. The method is proved to be valid in many extreme situations in both $2 \mathrm{D}$ and $3 \mathrm{D}$ cases.

It is worth noting that, for stationary boundaries, this identification process needs to be done just once at the beginning of the simulation and therefore represents only a small fraction of the total turnaround time. For moving boundaries, this identification process needs to be done at every time-step. However given that the immersed boundary can only travel a distance of the order of the nominal grid spacing in one time-step, the cell 
information from the previous time-step can be used to minimize the number of grid nodes for which the above process has to be carried out. Thus, even in the moving boundary case, the process only takes a very small fraction of the total CPU time. Consequently, very fine surface meshes can be used to provide highly accurate representations of the immersed geometry without any significant implications for the overall computational processing time.

\subsubsection{Face Velocity Calculation}

After all cells are successfully identified as solid or fluid cells, the stair boundaries can be determined. We only consider the solid bodies here. For solid bodies, the stair boundaries are the demarcations between solid and fluid cells. The boundary conditions, which are the face velocities normal to the cell faces, on those stair boundaries need to be imposed. In order to do that, ghost cells are identified first. The ghost cell are solid cells which close to the stair boundaries. As shown in Figure 2-13, for a 2D case, if at least one of the four faces of a solid cell is a stair boundary, the solid cell is a ghost cell. The approach now is to determine an appropriate equation for these ghost cells which leads to the implicit satisfaction of the boundary condition on the immersed boundary in the vicinity of each

ghost cell. We do this by extending a line segment from the node of these cells into the fluid to an "image point" (denoted by IP) such that it intersects normal to the immersed boundary and the boundary intercept (denoted by BI) is midway between the ghost node and the image point. 


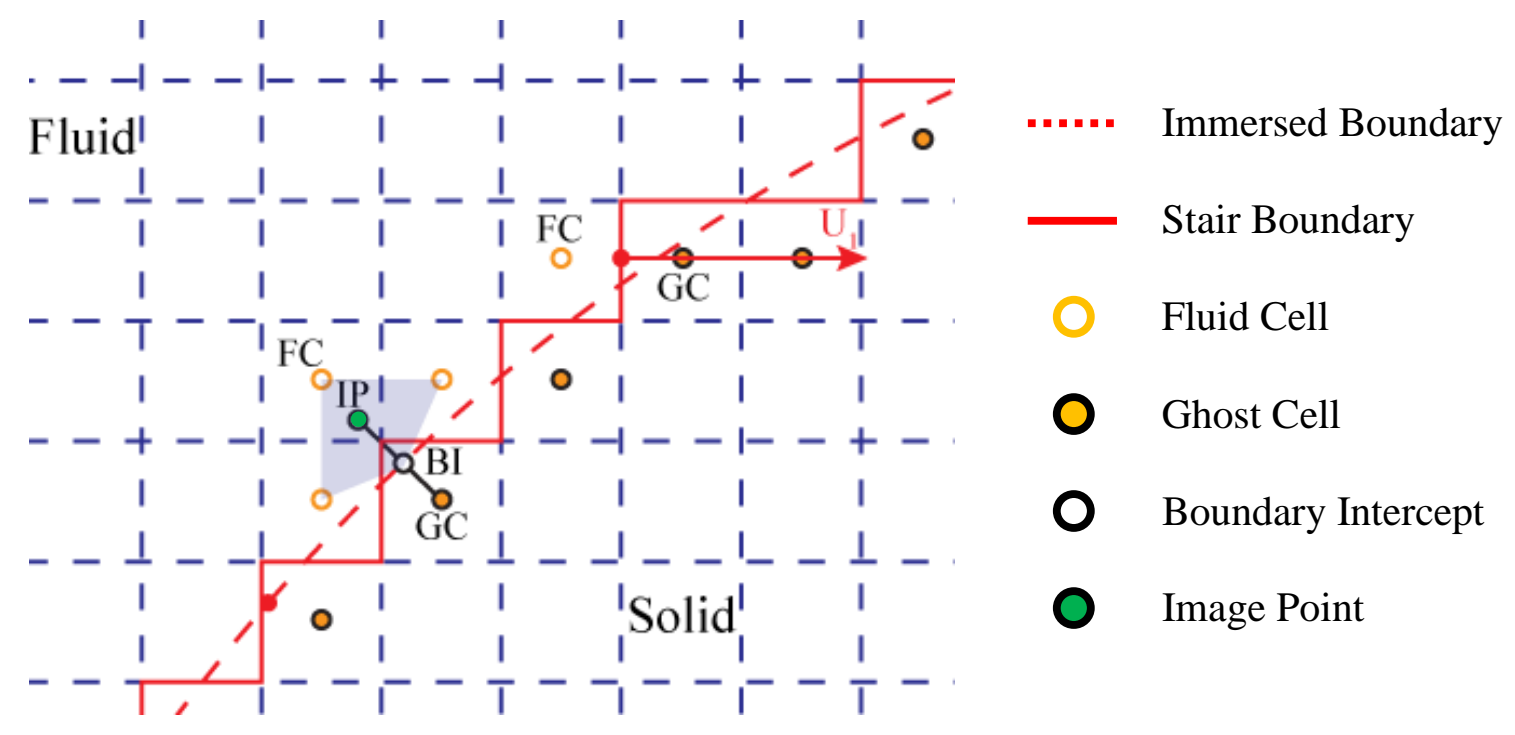

Figure 2-13. 2D schematic describing the stair boundary and ghost-cell methodology used in the current solver.

Once the BI and the corresponding IP have been identified, a trilinear interpolation in the following form is applied to calculate the velocity at the IP, and we take the velocity in $\mathrm{x}$ direction, $u_{I P}$, as an example:

$$
\begin{aligned}
& u_{I P}\left(x_{1}, x_{2}, x_{3}\right)=C_{1} x_{1} x_{2} x_{3}+C_{2} x_{1} x_{2}+C_{3} x_{2} x_{3} \\
& +C_{4} x_{1} x_{3}+C_{5} x_{1}+C_{6} x_{2}+C_{7} x_{3}+C_{8}+O\left(\Delta^{2}\right)
\end{aligned}
$$

The eight unknown interpolation coefficients can be determined in terms of the velocities of the eight surrounding nodes in 3D cases:

$$
\left\{C_{1}, C_{2}, \ldots, C_{8}\right\}^{T}=[V]^{-1}\left\{u_{1}, u_{2}, \ldots, u_{8}\right\}^{T}
$$


where $u_{i}$ are velocities of surrounding nodes and $[V]$ is the Vandermonde matrix [98] corresponding to the trilinear interpolation scheme shown in Equation(15) and has the form:

$$
[V]=\left[\begin{array}{cccccccc}
\left(x_{1} x_{2} x_{3}\right)_{1} & \left(x_{1} x_{2}\right)_{1} & \left(x_{1} x_{3}\right)_{1} & \left(x_{2} x_{3}\right)_{1} & \left(x_{1}\right)_{1} & \left(x_{2}\right)_{1} & \left(x_{3}\right)_{1} & 1 \\
\left(x_{1} x_{2} x_{3}\right)_{2} & \left(x_{1} x_{2}\right)_{2} & \left(x_{1} x_{3}\right)_{2} & \left(x_{2} x_{3}\right)_{2} & \left(x_{1}\right)_{2} & \left(x_{2}\right)_{2} & \left(x_{3}\right)_{2} & 1 \\
\vdots & \vdots & \vdots & \vdots & \vdots & \vdots & \vdots & \vdots \\
\left(x_{1} x_{2} x_{3}\right)_{8} & \left(x_{1} x_{2}\right)_{8} & \left(x_{1} x_{3}\right)_{8} & \left(x_{2} x_{3}\right)_{8} & \left(x_{1}\right)_{8} & \left(x_{2}\right)_{8} & \left(x_{3}\right)_{8} & 1
\end{array}\right]
$$

where the subscripts outside the parentheses are identifiers of the eight surrounding nodes in $3 \mathrm{D}$ cases. It is worth noting that the interpolation scheme is proven to be $2^{\text {nd }}$ order accurate.

After the velocity at the IP is obtained, the velocity at the GC can be calculated as follows:

$$
u_{G C}=2 u_{B I}-u_{I P}
$$

And the face velocity at the stair boundary can be calculated as:

$$
U_{1}=\frac{1}{2}\left(u_{F C}+u_{G C}\right)
$$

\subsubsection{Conflicting Face Velocity Correction and Gap Filling between Solid and Membrane Bodies}

We have successfully imposed boundary conditions for solid bodies. However, there exists situations as "a solid body with attaching membrane bodies" in many biological flapping systems. Just as shown in Figure 2-11(a), the baby trout fins are firmly attached 
to the trunk body. In this section, a new approach is introduced to overcome the computational difficulties regarding such situations.

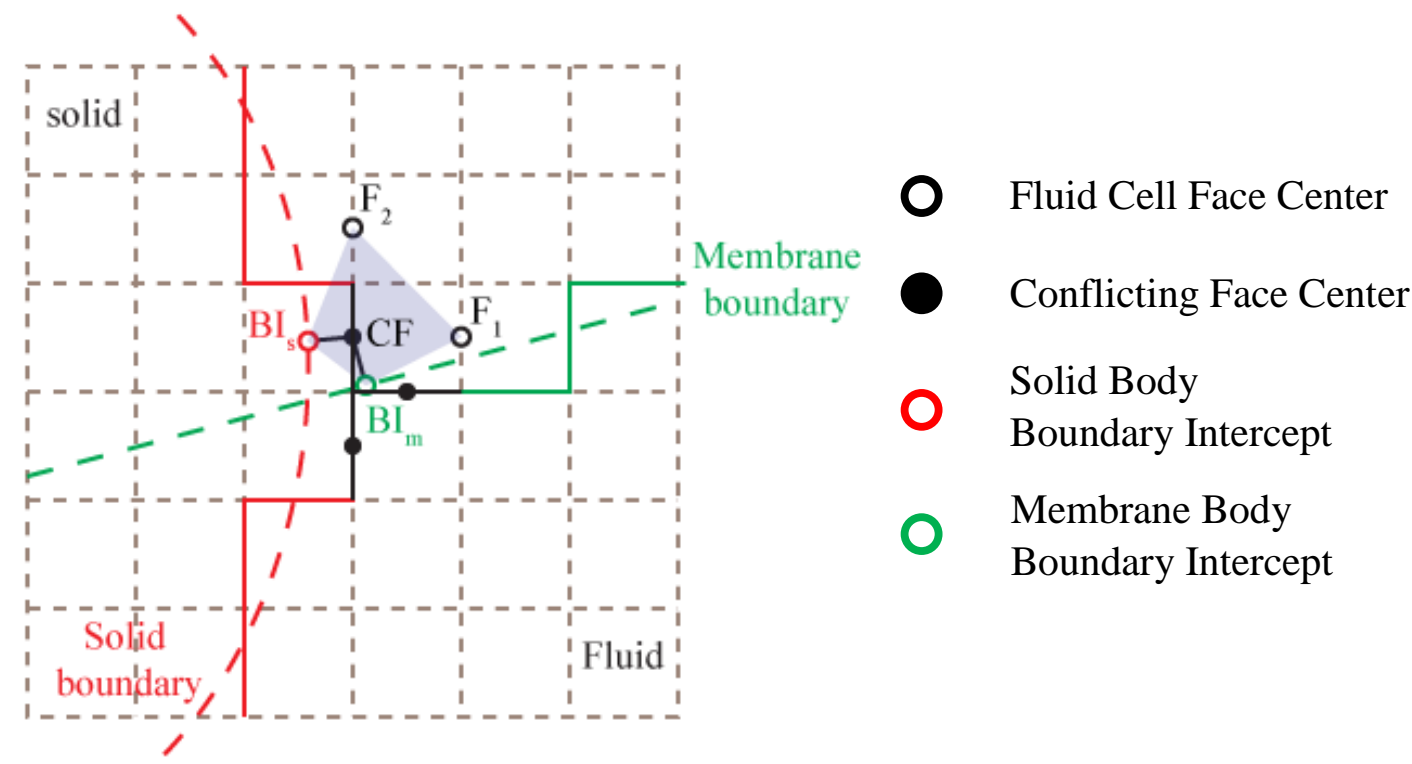

Figure 2-14. 2D schematic describing the methodology which handles deformable attaching bodies.

Figure 2-14 shows a 2D schematic plot of the approach. The dashed lines are immersed boundaries and the solid lines are the corresponding stair boundaries. For the membrane body, the stair boundaries are chosen as the closest fluid cell faces to the elements on the immersed boundary. As shown in the figure, several conflicting faces can be identified. The conflicting faces are stair boundaries which directly connect to the intersection of the solid and the membrane stair boundaries. The boundary conditions on the conflicting faces need special treatments. The velocity of one conflicting face may be calculated according to a reference velocity of the solid immersed boundary at one time step. For the next time 
step, the reference velocity may be altered to the velocity of the membrane immersed boundary due the motion of the moving boundaries. This will cause a discontinuity of the velocity boundary condition on the conflicting face for those two time steps, which will cause numerical instability and eventually lead to the divergence of the flow solver.

In order to solve the above problem, the velocities of the conflicting faces are corrected accordingly. Here we describe the method using the upper conflicting face (Figure 2-14) as an example. From the center of the conflicting face, we first drop two lines perpendicular to the solid and membrane immersed boundaries. There we get two boundary intercept, $B I_{s}$ and $B I_{m}$. Another two fluid cell faces which are close to the conflicting face can be found, and the corresponding face centers are $F_{1}$ and $F_{2}$. An interpolation stencil (shaded area) is formed according to the four points. In 3D cases, the stencil consists of eight points (two boundary intercept and six fluid cell face centers). The conflicting face velocity can then be calculated as follows:

$$
\begin{aligned}
& U_{C F}\left(x_{1}, x_{2}, x_{3}\right)=C_{1} x_{1} x_{2} x_{3}+C_{2} x_{1} x_{2}+C_{3} x_{2} x_{3} \\
& +C_{4} x_{1} x_{3}+C_{5} x_{1}+C_{6} x_{2}+C_{7} x_{3}+C_{8}+O\left(\Delta^{2}\right)
\end{aligned}
$$

The eight unknown interpolation coefficients can be determined in terms of the face velocities of the eight points of the interpolation stencil:

$$
\left\{C_{1}, C_{2}, \ldots, C_{8}\right\}^{T}=[V]^{-1}\left\{U_{1}, \mathrm{U}_{2}, \ldots, \mathrm{U}_{8}\right\}^{T}
$$

where $U_{i}$ are the face velocities at the interpolation stencil points and $[V]$ is the Vandermonde matrix shown in Equation(17). This process continues until the velocities of all conflicting faces are corrected. 


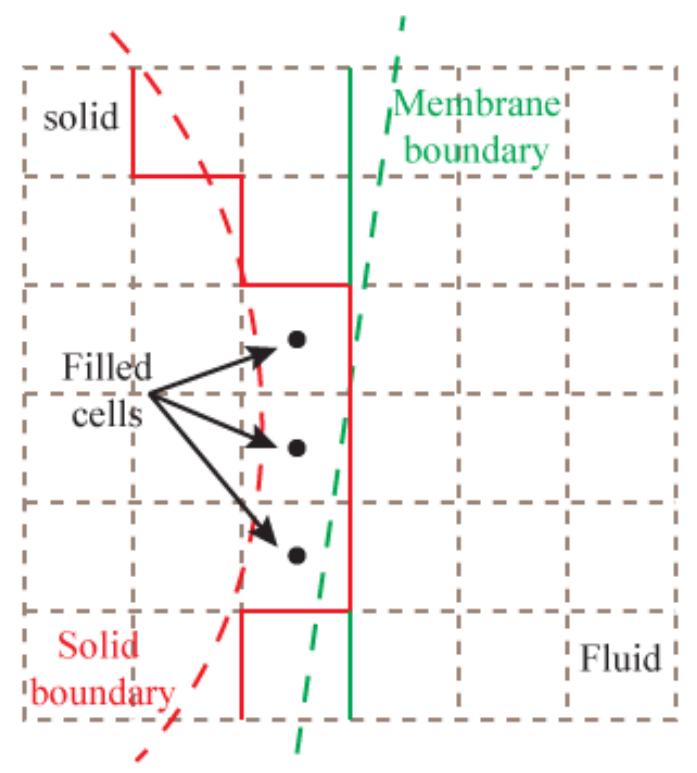

Figure 2-15. 2D schematic describing the methodology which handles very close solid body and membrane body.

Another extreme situation is shown in Figure 2-15, where the two types of the immersed boundaries are too close to each other. The solver diverges in this situation due to the instability of the boundary conditions at the narrow passage between the two boundaries. In order to solve the problem, we measure the minimum distances of the narrow passage, and fill the gaps if the distance is less than one cell width. Three filled cells can be identified in Figure 2-15 according to this rule, and the stair boundaries are modified based on those filled cells. The conflicting faces still need to be identified and the corresponding velocities need to be corrected based on the modified stair boundaries. 


\subsubsection{Validation: Steady Swimming of a Baby Trout}

Full body direct numerical simulation of a steady swimming baby trout is conducted. This case serves as validation for both the IBM-DAB method and the joint-based surface reconstruction method introduced in section 2.1. Figure 2-16 shows the comparison of the baby trout motion in high-speed images to the corresponding reconstructed model in three different views. We can see from the figure that the reconstruction is highly accurate. Three fins, the dorsal fin, anal fin, and caudal fin, along with the trunk body of the fish are included in the simulation. Those fins are firmly attached to the fish body, which need special treatment for imposing boundary conditions in the full body simulation. IBM-DAB is developed in the current work to serve the purpose.

Table 2-5. Baby trout steady swimming parameters.

\begin{tabular}{lc}
\hline Body length, $L(\mathrm{~cm})$ & 7.00 \\
Body height, $H(\mathrm{~cm})$ & 1.58 \\
Forward speed, $U(\mathrm{~cm} / \mathrm{s})$ & 9.32 \\
Flapping amplitude, $A(\mathrm{~cm})$ & 1.32 \\
Flapping frequency, $f$ & 3.70 \\
Reynolds number, $R e$ & 6521.9 \\
Strouhal number, $S t$ & 0.52 \\
\hline
\end{tabular}

Table 2-5 lists the parameters of the steady swimming baby trout. The flow conditions are strictly match with those parameters. Figure 2-11(b) shows the reconstructed realistic full-body model immersed in the three-dimensional non-uniform Cartesian grid. $Y$ is the vertical direction with $+Y$ pointing upward. The simulation domain size normalized by the 
fish body length $L$ is $10 \times 6 \times 6$ and this large domain ensures that the boundaries do not have any significant effect on the computed results. The computational grid adopted here has $384 \times 193 \times 193$ points. The largest grid size in the dense region is $6.875 \times 10^{-3}$ body length, corresponds to 145 grids across the body length.

(a)

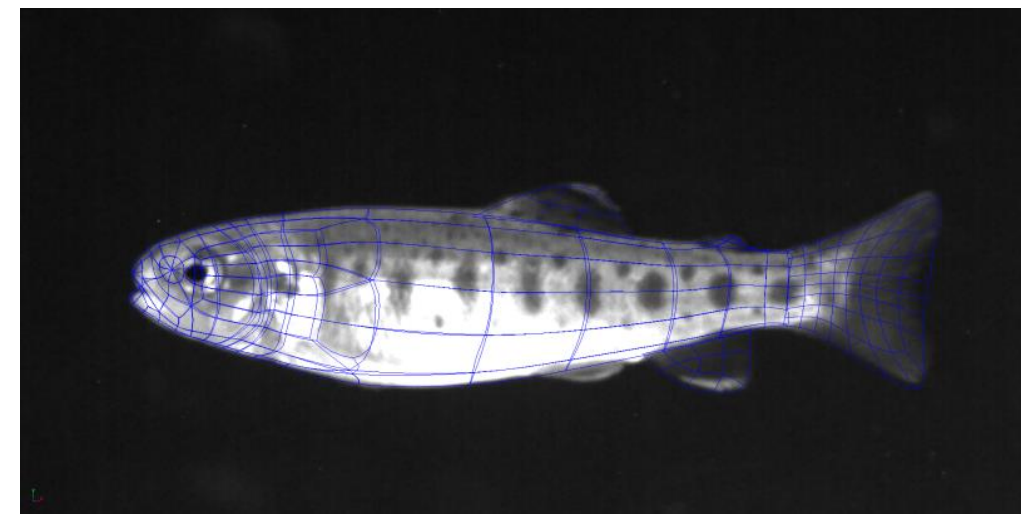

(b)

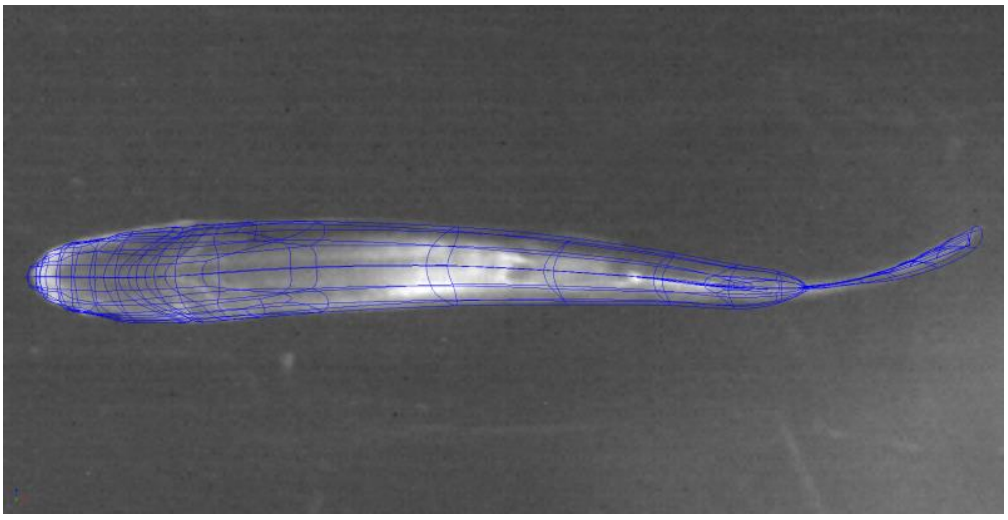

(c)

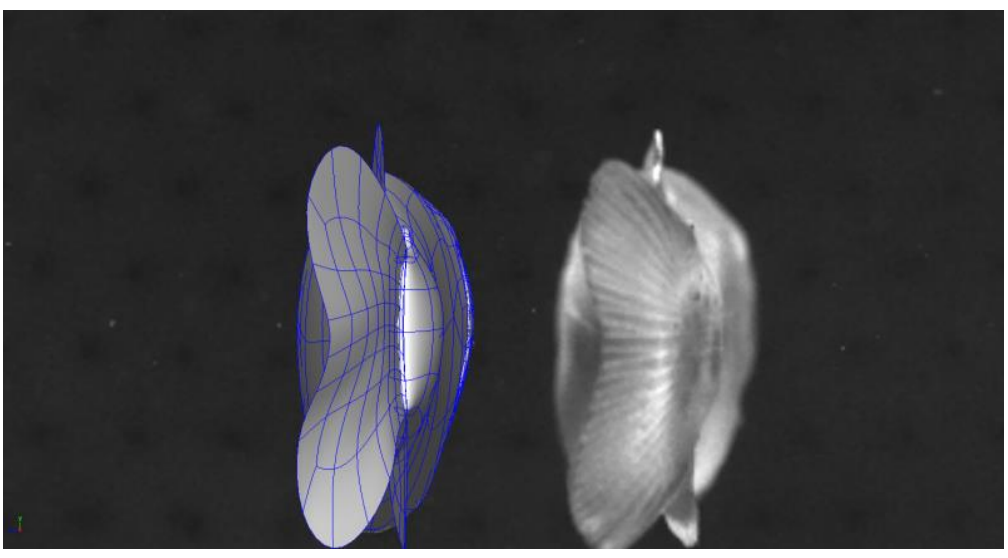

Figure 2-16. Comparison of the baby trout motion shown in high-speed images to the corresponding reconstructed model. (a) Lateral view; (b) ventral view; (c) back view. 
Figure 2-17 shows the time course of hydrodynamic performance of this case. The thrust and power generated by the dorsal and anal fins are much smaller comparing to that generated by the body and caudal fin of the trout. The caudal fin generates large thrust force for most of the time during the stroke, while the trout body show large drag production. Table 2-6 lists the cycle averaged hydrodynamic performance of this case. We can see that the caudal fin produce large amount of thrust while the other three parts (the body, dorsal fin, and anal fin) produce drag. The resultant thrust is very small and is about $3 \%$ of the thrust produced by the caudal fin, which validates the IBM-DAB method and the joint-based surface reconstruction method.
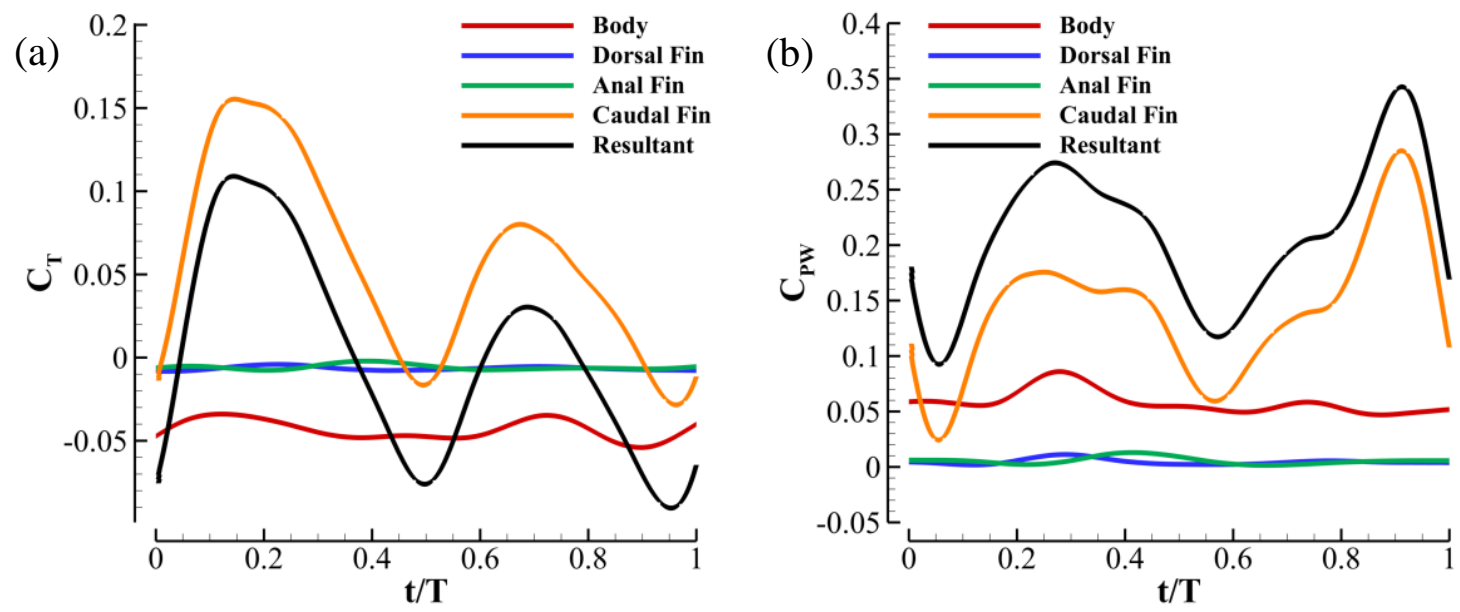

Figure 2-17. Time course of hydrodynamic (a) thrust and (b) power coefficient of the steady swimming baby trout.

Figure 2-18 shows the 3D wake structures in three different views of this validation case. We can see from the figure that the wake structures are pretty complicated and the computational grid is dense enough to resolve the flow field of this case. 
Table 2-6. Cycle averaged hydrodynamic performance

\begin{tabular}{lcc}
\hline & $\overline{C_{T}}\left(\times 10^{-3}\right)$ & $\overline{C_{P W}}\left(\times 10^{-3}\right)$ \\
\hline Body & -43.37 & 58.32 \\
Dorsal Fin & -6.48 & 4.63 \\
Anal Fin & -5.77 & 5.57 \\
Caudal Fin & 57.50 & 141.85 \\
\hline Resultant & 1.88 & 210.37 \\
\hline
\end{tabular}

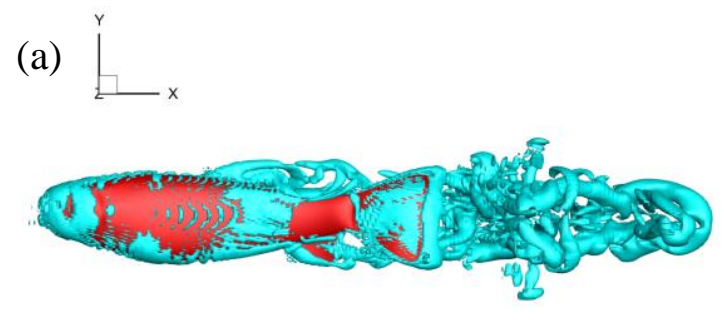

(b) $\stackrel{2}{L} x^{2}$

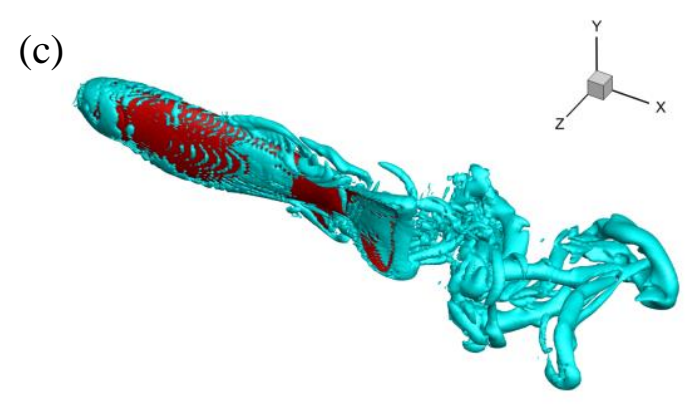

Figure 2-18. Three-dimensional wake structures of the steady swimming baby trout. (a) Lateral view; (b) ventral view; (c) perspective view. 


\subsection{Gradient-based Computational Optimization}

Conventional high-fidelity computational optimization methods $[81,86]$ are costly, especially for 3D cases. In order to keep durability, one has to compromise between model fidelity and computational effort. Here, a gradient-based parallel curve searching optimizer is developed in the current work. A computational optimization frame (Figure 2-19), which consist of a kinematics generator, a high-fidelity flow solver, and the parallel curve searching optimizer, is built. The computational cost of finding the searching direction and the step size are greatly reduced with the help of this frame.

The first two parts of the optimization frame contain computational models of flapping propulsors (mathematical propulsor models or low dimensional models from SSVD analysis) and evaluations of aero/hydrodynamic performance via direct numerical simulations (DNS), while the third part calculates searching direction and step size. The procedure starts with perturbing design variables (simplified morphological parameters or SSVD modes amplitudes/phases) to calculate searching direction. This step is time consuming due to our high-fidelity aero/hydrodynamic performance evaluation method (DNS). Hence a parallel computing algorithm is applied to greatly reduce the computational cost. The parallel computing algorithm is the best fit to our problems since the number of design variables is limited by either building simplified mathematical propulsor models or constructing low dimensional models based on dominant SSVD modes. Optimization step size is then determined by a newly developed inexact curve searching (ICS) method, which also greatly reduce the computational cost comparing to the conventional line search method. Those design variables are then updated and sent to 
DNS solver for the next iteration. This process is continued until an optimal design is obtained.

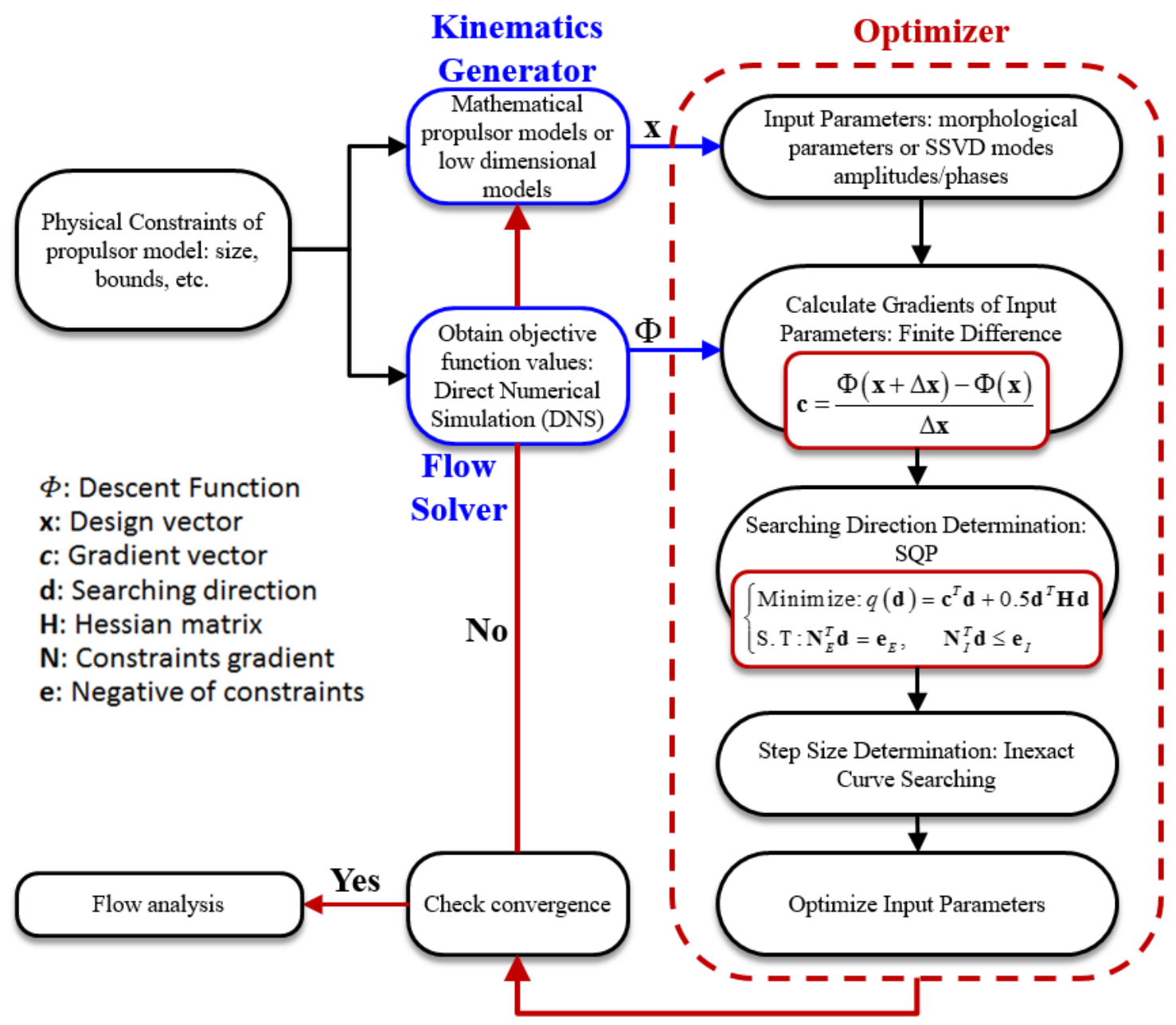

Figure 2-19. A schematic of the computational optimization frame.

\subsubsection{Sequential Quadratic Programming (SQP)}

Sequential quadratic programming method is used to obtain the searching direction of the optimization process. We start by defining the decent functions as combinations of 
aerodynamic performance from DNS solver $\left(\overline{C_{F}}\right.$ or $\overline{C_{F}} / \overline{C_{P W}}$ ) and penalties from constraints of design variables:

$$
\Phi_{i}(\mathbf{x})=\left\{\begin{array}{lc}
-\overline{C_{F}}+R V(\mathbf{x}) & i=1 \\
-\overline{C_{F}} / \overline{C_{P W}}+R V(\mathbf{x}) & i=2
\end{array}\right.
$$

where $i$ denotes the case number ( $i=1$ for aero/hydrodynamic force optimization and $i=2$ for propulsive efficiency optimization), $\mathbf{x}$ is the design vector, $\overline{C_{F}}$ and $\overline{C_{P W}}$ denote average aero/hydrodynamic force coefficient and power coefficient over several flapping cycles, $R$ is a strictly positive number called the penalty parameter (initially specified by the user) and may change during the iterative process, $V(\mathbf{x})$ is either the maximum constraint violation defined as:

$$
V=\max \left\{0 ;\left|\mathrm{h}_{1}\right|,\left|\mathrm{h}_{2}\right|, \ldots,\left|\mathrm{h}_{p}\right| ; g_{1}, g_{2}, \ldots, g_{m}\right\}
$$

where $h$ and $g$ are equality and inequality constraint functions, the subscripts denote the index of those constraint functions. In subsequent iterations, the penalty parameter $R$ is updated flowing the rules:

$$
\left\{\begin{array}{l}
r=\sum_{i=1}^{p}\left|v_{i}\right|+\sum_{i=1}^{m}\left|u_{i}\right| \\
R=\max (R, r)
\end{array}\right.
$$

where $r$ is the sum of all of the Lagrange multipliers $\left(u_{i}\right.$ and $\left.v_{i}\right)$.

The searching direction is then determined by quadratic programing (QP) sub-problem, which itself is an analytical minimization problem: 


$$
\begin{array}{ll}
\text { Minimize: } & \bar{f}=\mathbf{c}^{T} \mathbf{d}+\frac{1}{2} \mathbf{d}^{T} \mathbf{H d} \\
\text { subject to: } & \left\{\begin{array}{l}
\mathbf{N}^{T} \mathbf{d}=\mathbf{e} \\
\mathbf{A}^{T} \mathbf{d} \leq \mathbf{b}
\end{array}\right.
\end{array}
$$

where $\bar{f}$ represents the cost function of this sub-problem, $\mathbf{d}$ is the searching direction to be solved, $\mathbf{c}$ denotes gradient vector, $\mathbf{N}$ and $\mathbf{A}$ are gradient matrices of equality and inequality constraint functions respectively, $\mathbf{e}$ and $\mathbf{b}$ are negative values of equality and inequality constraint functions, $\mathbf{H}$ denotes hessian matrix. Equation(25) can be solved along with Lagrange Multiplier u using KKT optimality condition.

The gradient vector $\mathbf{c}$ of the objective function is given by:

$$
\mathbf{c}=\nabla \Phi_{i}=\left(\frac{\partial \Phi_{i}}{\partial x_{1}}, \frac{\partial \Phi_{i}}{\partial x_{2}}, \frac{\partial \Phi_{i}}{\partial x_{3}} \ldots . .\right)
$$

where $x_{k}$ are the components of design vector $\mathbf{x}$ (design variables). The components of the gradient vector are then evaluated numerically by computing the objective function for a perturbation of all of the design variables one at a time. Note that the evaluation of these vector components requires an unsteady flow solution over a few periods of the flapping motion until a periodic flow behavior is reached. The Hessian matrix $\mathbf{H}$, which contains $2^{\text {nd }}$ order derivative information of the objective function defined in equation(22), is approximated and updated by Quasi-Newton Hessian Approximation (QNHA) method [99] for each optimization iteration.

Once the desirable searching direction $\mathbf{d}$ is obtained by solving the minimization problem defined in equation(25), a small step in this direction is taken to update the design variables. The step size is determined by step size sub-problem, in which a newly 
developed inexact curve searching method (ICS) is applied. The whole process is continued in an iterative manner until a local minimum is reached.

\subsubsection{Inexact Curve Searching (ICS)}

The conventional line search method for determining the step size can be quite timeconsuming, especially when using a high-fidelity performance evaluation tool (DNS solver). Therefore, an inexact curve searching (ICS) method is developed in the current work. The basic concept of the method is that the step size should not be too large or too small, and there should be a sufficient decrease in the cost function value along the searching direction.

After the searching direction is obtained, an initial step size is determined as:

$$
\alpha_{0}=\delta \sqrt{\sum_{i=1}^{n}\left(b_{u}^{i}-b_{l}^{i}\right)^{2}} /|\mathbf{d}|
$$

where $\alpha_{0}$ is the initial step size, $b_{u}^{i}$ denotes the upper bound of the $i^{\text {th }}$ design variable, $b_{l}^{i}$

denotes the lower bound of the $i^{\text {th }}$ design variable, $n$ stands the total number of design variables, $\mathbf{d}$ is the searching direction vector, $\delta$ is the initial step size ratio, which is chosen as 0.1 for the cases in the current work. The initial step size is then substitute to the following equation to evaluate its validity:

$$
\Phi\left(\mathbf{x}+\alpha_{0} \mathbf{d}\right) \leq \Phi(\mathbf{x})-\alpha_{0} \gamma|\mathbf{d}|
$$


where $\Phi$ is the decent function defined in equation(22), $\gamma$ is a specified constant between 0 and 1 , which is chosen as 0.5 in the current work. If equation(28) is true, $\alpha_{0}$ is the desired step size. Otherwise, a new searching direction $\mathbf{d}^{*}$ is recalculated using the updated design vector $\mathbf{x}^{*}=\mathbf{x}+\alpha_{0} \mathbf{d}$. Also, a new step size is updated as $\alpha_{0}^{*}=\alpha_{0} / 2$. The $\mathbf{x}^{*}, \mathbf{d}^{*}$, and $\alpha_{0}^{*}$ are then substituted to equation(28) to check the validity of $\alpha_{0}^{*}$. The process is iterated until equation(28) is evaluated to be true, and the desired step size is found then.

\subsubsection{Validation: Optimization of a 2D Hovering Plate with Dynamic Trailing-Edge Flap on Lift Production}

In this section, computational optimization of a $2 \mathrm{D}$ hovering plate with dynamic trailing-edge flap is performed. The problem definition regarding the plate modeling and the corresponding computational setup can be found in previous work done by $\mathrm{Li}$, et al. [100]. The objective function of the validation case is chosen to be the cycle averaged lift coefficient $\overline{C_{L}}$, and the design variables are the trailing-edge flap deflection amplitude $\beta$ and the corresponding phase difference with respect to the leading-edge pitching motion. The optimization frame is validated by comparing the optimal configuration of the design variables with the results of separately performed parametric studies.

Figure 2-20 shows the parametric results along with the optimization convergence histories for comparison. Two optimization cases (Start 1 and Start 2) with different initial guesses of the design variables are performed. We can see from the figure that both cases

converges to the same optimum location, which is exactly where the maximum $\overline{C_{L}}$ can be 
found in the parametric studies contour. The paths of the convergence histories show curved shapes, and the number of iterations and performance evaluations are similar (around 12 for both cases). Those are due to the nature of the ICS method applied in the current optimizer. For the conventional line search algorithms, the number of performance evaluations are around 50 for this case. The computational cost is greatly reduced with the help of the current optimization frame.

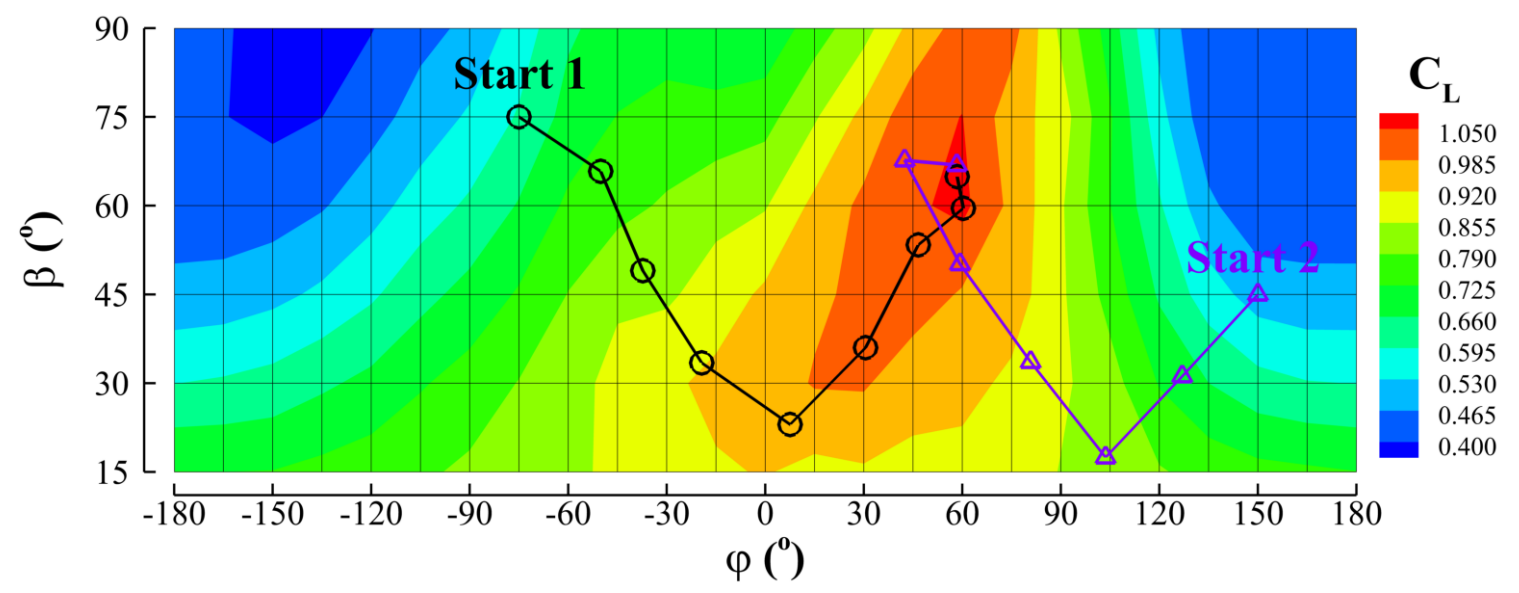

Figure 2-20. Parametric study results showing the effect of trail-edge flap deflection amplitude $\beta$ and the corresponding phase difference $\varphi$ on the aerodynamic lift production. The optimization convergence histories are also shown for comparison. 


\section{Computational Optimization of Flexible Wing}

\section{Aerodynamic Performance in Flapping Motion}

The recent surge of interest in the development of micro aerial vehicles has led to several efforts to develop prototype micro-flapping robotic devices inspired by insects or birds. However, design of high-performance wings is still under investigation. For example, cambered wings have been observed in many animal flights, it refers to the asymmetry between the top and bottom curves of a wing in cross-section. Among flying animals, birds and bats can actively camber their wings for aerodynamic force modulation [101, 102]. Insect wings also demonstrate a variety passive cambering patterns as a result of wing flexural stiffness, kinematics, and fluid-structure interactions [103, 104]. However, studies on the optimal dynamic change of wing camber and its unsteady aerodynamic effect are very limited and lack systematic approaches. In this work, a direct numerical simulation (DNS) based computational optimization frame is developed to investigate the optimal setting of wing camber parameters during a leading-edge driven flapping flight. In details, a finite-difference based immersed boundary solver for incompressible flows is used to calculate the aerodynamic performance of the 2D flapping wing and its unsteady flows. A gradient-based optimization frame is used to determine the optimal morphological parameters of flapping wings to achieve the desired wing performance. Results aim to provide insights into the effect of camber and its optimal formation for a wing in flapping motion. 


\subsection{Problem Definition}

A gradient based non-linear optimizer has been developed to get the optimal configurations of wing morphological parameters (design variables) which can have maximum thrust production or propulsive efficiency. A second-order finite-difference based immersed-boundary solver [97] is used to get those aerodynamic forces. Sequential quadratic programming method is used to get the searching direction and inexact curve searching method is used to get the searching step size. As shown in Figure 2-19, the optimization chain includes three major parts: a DNS solver (to evaluate the cost function), a kinematics generator, and an optimizer. The procedure is based on the maximization of mean thrust production or propulsive efficiency. This is achieved by the modification of design variables such as pivot position, trailing edge deflection amplitude, or phase of the trailing edge deflection. The optimizer provides the testing value of design variables to the kinematics generator to get the inputs of the DNS solver. Aerodynamic performance is then evaluated and used as the value of cost function. This process is continued until an optimal design is obtained.

Figure 3-1 shows a schematic plot of the flexible wing modeling. The flapping wing kinematics is defined as:

$$
\left\{\begin{array}{l}
y(t)=A_{y} \cos (2 \pi f t) \\
\alpha(t)=\alpha_{m} \sin (2 \pi f t)
\end{array}\right.
$$

where $f$ is the flapping frequency, $y$ is the leading-edge (LE) translation, $\alpha$ is the LE pitching angle, $A_{y}$ is the LE flapping amplitude, $\alpha_{m}$ is the LE pitching amplitude. The dynamic wing morphing is modeled by adding a pivot point, about which the trailing-edge 
(TE) portion of the wing is rotate. The location of the pivot point and the corresponding dynamic wing morphing are defined as:

$$
\left\{\begin{array}{l}
P_{R}=A B /(A B+B C) \\
\beta(t)=\beta_{m} \sin (2 \pi f t+\varphi)
\end{array}\right.
$$

where $P_{R}$ is the pivot ratio, $\beta$ is the TE deflection angle, $\beta_{m}$ is the TE deflection amplitude, $\varphi$ is the TE deflection phase difference with respect to the LE pitching motion. Three design variables describing the morphing wing kinematics are chosen as $P_{R}, \beta_{m}$, and $\varphi$ for the current work. It is worth noting that the curved wing shape, which is obtained via polynomial fitting of the LE, Pivot point, and the TE, is applied in the simulations.

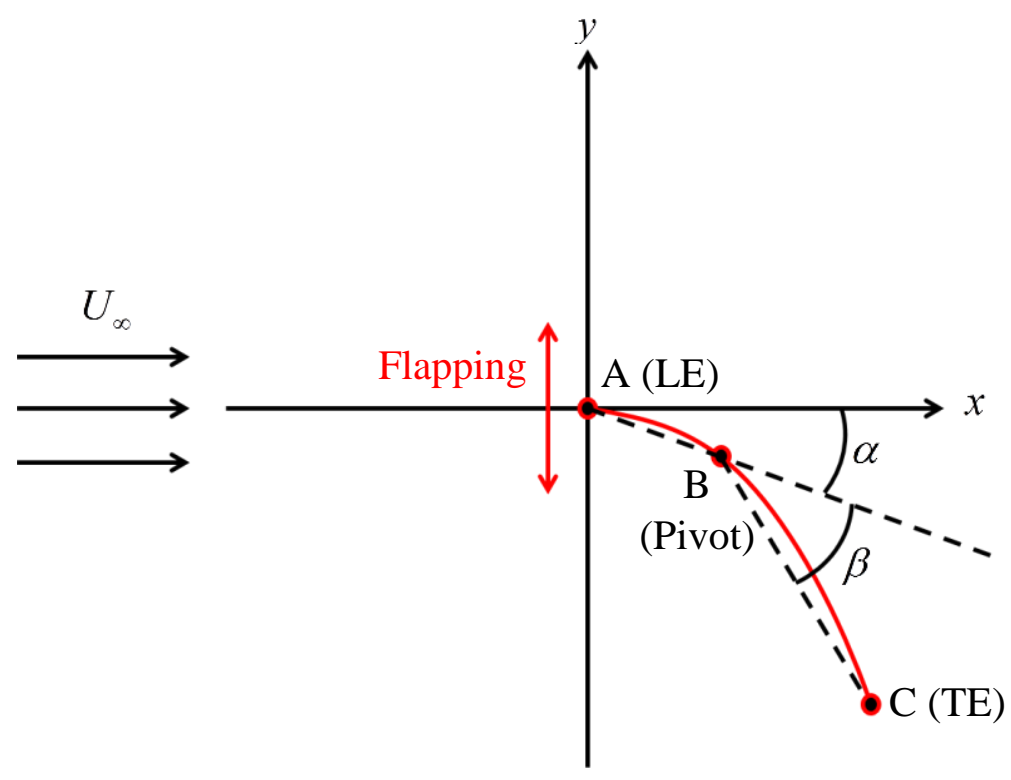

Figure 3-1. Schematic plot showing the modeling of the flexible wing. 
The Reynolds number and Strouhal number applied in the current work are 200 and 0.6 , respectively. The definition of the two non-dimensional numbers are:

$$
\left\{\begin{array}{l}
R e=U_{\infty} c / v \\
S t=2 A_{y} f / U_{\infty}
\end{array}\right.
$$

where $c$ is the wing chord length, $v$ is the kinematic viscosity of the fluids. The aerodynamic performance of the wings is evaluated through thrust coefficient and power coefficient, which are defined as follows:

$$
C_{T}=\frac{F_{T}}{1 / 2 \rho U_{\infty}^{2} c}, \quad C_{P W}=\frac{P}{1 / 2 \rho U_{\infty}^{3} c}
$$

where $F_{T}$ is the thrust force, $P$ is the aerodynamic power, $\rho$ is the fluid density. As mentioned before, the thrust production and propulsive efficiency of the wings are optimized in the current work, which are the cycle averaged thrust coefficient $\left(\overline{C_{T}}\right)$ and propulsive efficiency $\left(\eta=\overline{C_{L}} / \overline{C_{P W}}\right)$.

\subsection{Optimization of Thrust Production and Propulsive Efficiency}

Optimizations of thrust production and propulsive efficiency are performed in this section. Figure 3-2 and Figure 3-3 show the convergence histories of the design variables of these two optimization cases, which are denote as Opt $C_{T}$ and Opt $\eta$, respectively. Two initial guesses are applied in the current work for both cases, and they lead to the same optimum design variables for both cases. The numbers of iterations are around 10 , and the numbers of performance evaluations are around 12 for all cases discussed here. 
(a)

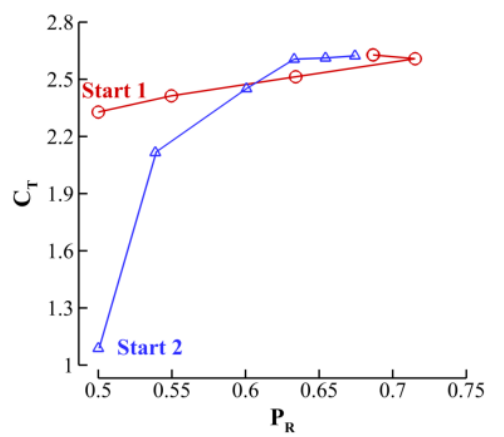

(b)

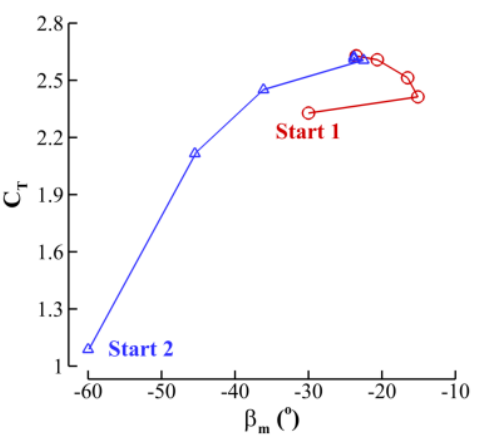

(c)

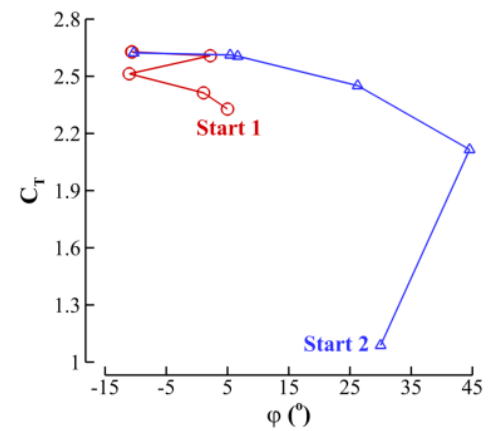

Figure 3-2. Convergence histories of design variables (a) $P_{R}$, (b) $\beta_{m}$, and (c) $\varphi$ of case Opt $C_{T}$.

(a)

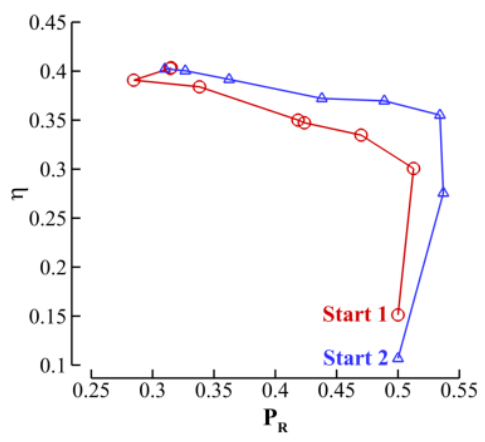

(b)

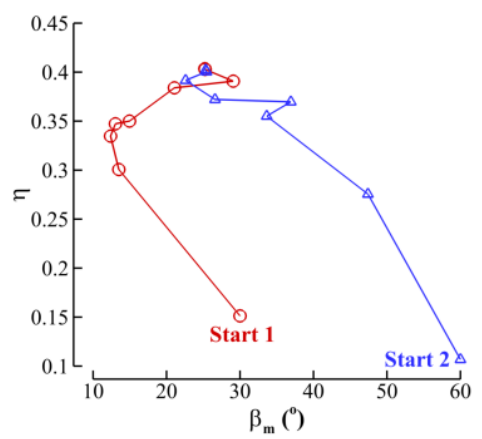

(c)

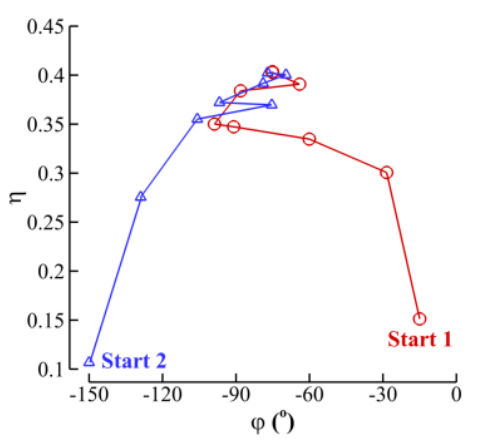

Figure 3-3. Convergence histories of design variables (a) $P_{R}$, (b) $\beta_{m}$, and (c) $\varphi$ of case Opt $\eta$.

Figure 3-4 shows the time histories of aerodynamic performance of the two optimization cases along with a completely rigid wing case for comparison. We can see that the case Opt $C_{T}$ shows largest amplitude of the thrust production among the three 
cases, while the case Opt $\eta$ presents lowest of that. However, the case Opt $\eta$ shows lowest power consumption. It is worth noting that the aerodynamic performance are exactly the same during the downstroke and upstroke of the flapping motion for both optimum cases, while that show slightly difference for the Rigid case.

(a)

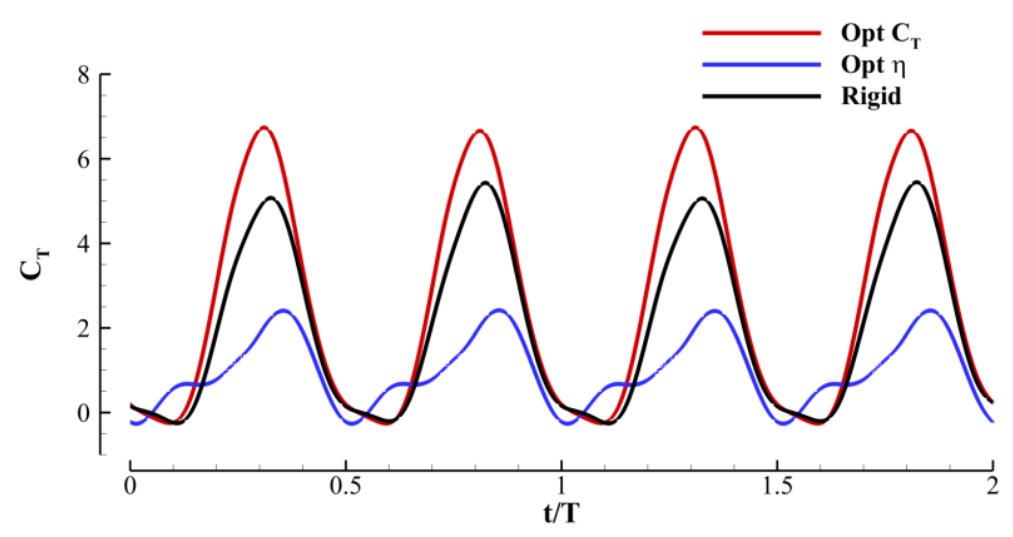

(b)

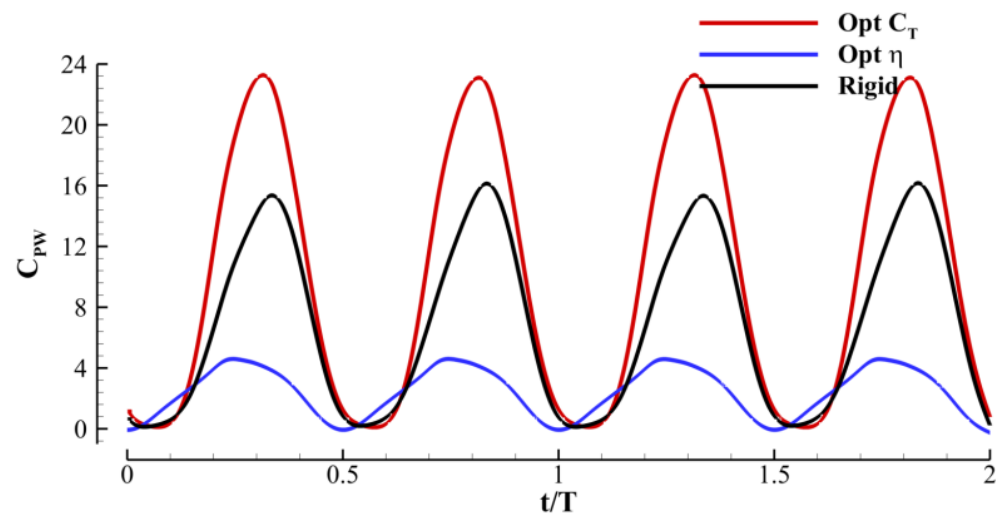

Figure 3-4. Time courses (a) thrust coefficients and (b) power coefficients for the cases Opt $C_{T}$, Opt $\eta$, and Rigid.

Table 3-1 lists the cycle averaged aerodynamic performance for the two optimum cases and the case Rigid. Comparing to the case Rigid, the thrust production of the Opt $C_{T}$ case is increased by $29.1 \%$, and the propulsive efficiency of the Opt $\eta$ case is increased by 
$36.2 \%$. Dynamic camber formation can greatly improve the aerodynamic performance of the flapping wing.

Table 3-1. Cycle averaged aerodynamic performance.

\begin{tabular}{lcccccc}
\hline \hline Cases & $P_{R}$ & $\beta_{m}\left({ }^{\circ}\right)$ & $\varphi\left(^{\circ}\right)$ & $\overline{C_{T}}$ & $\overline{C_{P W}}$ & $\eta$ \\
\hline Opt $C_{T}$ & 0.687 & -23.5 & -10.6 & 2.62 & 10.3 & 0.254 \\
\hline Opt $\eta$ & 0.315 & 25.2 & -75.1 & 0.968 & 2.45 & 0.395 \\
\hline Rigid & N/A & 0 & N/A & 2.03 & 6.99 & 0.290 \\
\hline
\end{tabular}

\subsection{Wake Structures of the Optimized Wing Models}

The unsteady wake structures of the optimum cases and the case Rigid are discussed in this section. Figure 3-5 shows the corresponding results. We can see from Figure 3-5(a) that the case Opt $C_{T}$ shows a typical $2 \mathrm{~S}$ wake structure. Two counter rotating vortices are generated in a complete wing stroke. The two vortices induce a local flow jet points to the downstream direction, thus produce thrust force. Differently, for the case Opt $\eta$ (Figure 3-5b), a typical $2 \mathrm{P}$ wake structure can be identified. Two pairs of counter rotating vortices, which induce two local flow jets pointing to the downstream direction, are generated in a complete wing stroke. The lower and upper vortex streets of the two optimization cases present symmetry property, which can be related to the symmetry aerodynamic performance of the two cases shown in Figure 3-4. For the case Rigid, a $2 \mathrm{~S}$ wake structure with asymmetric property can be observed. In each stroke, the two counter rotating vortices 
induce a flow jet more pointing to the vertical direction, which degrade the propulsive performance.

(a)
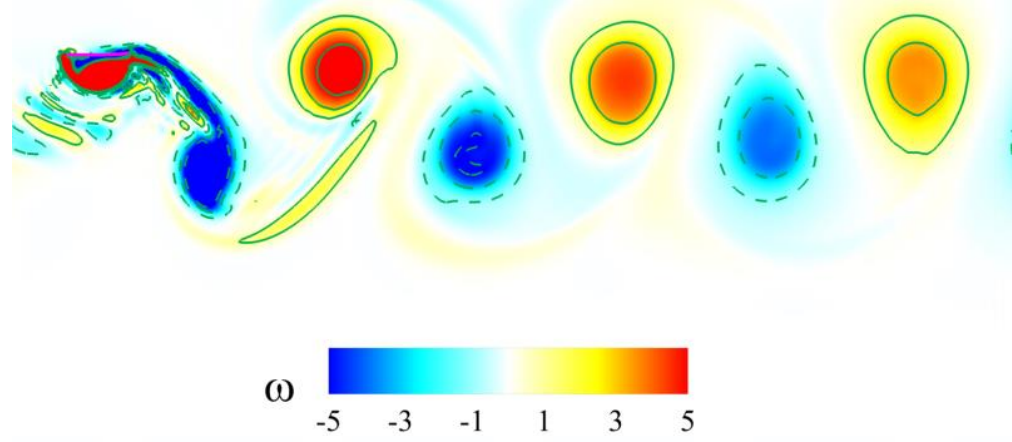

(b)

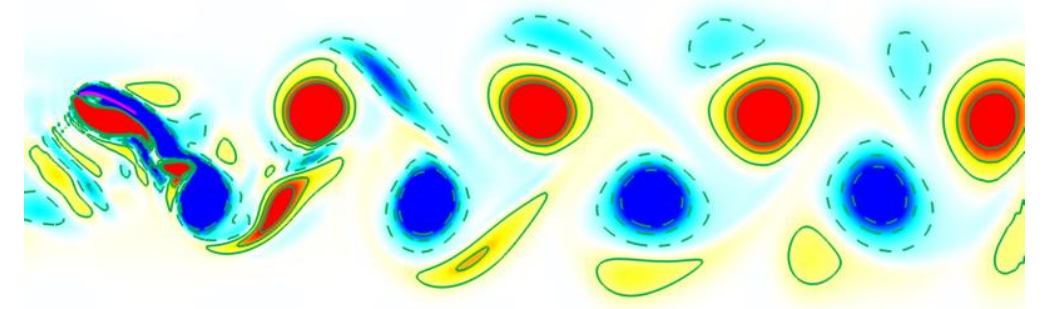

(c)
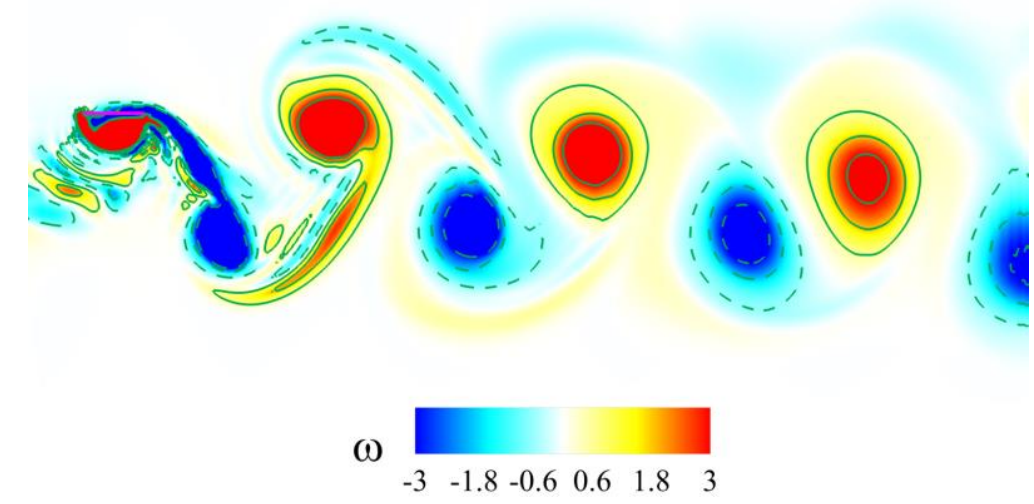

Figure 3-5. Wake structures of the cases (a) Opt $C_{T}$, (b) Opt $\eta$, and (c) Rigid. The contours show the normalized vorticity. 
(a)
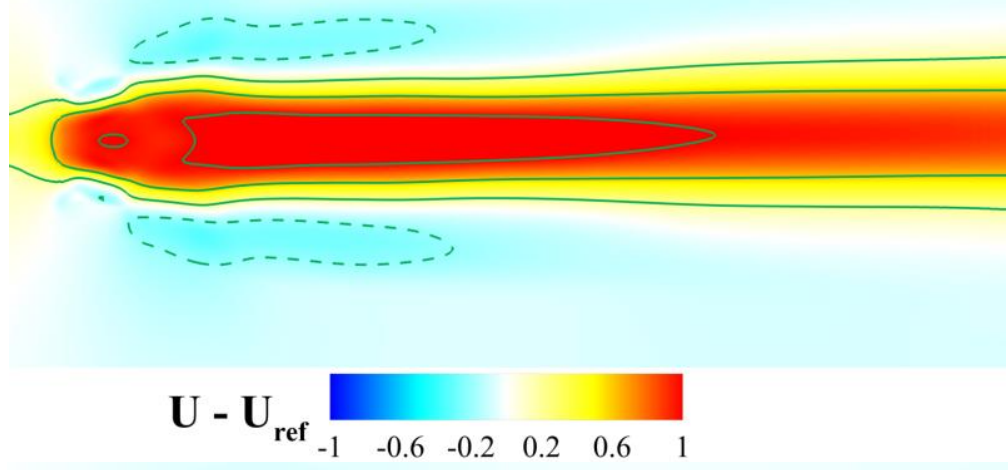

(b)
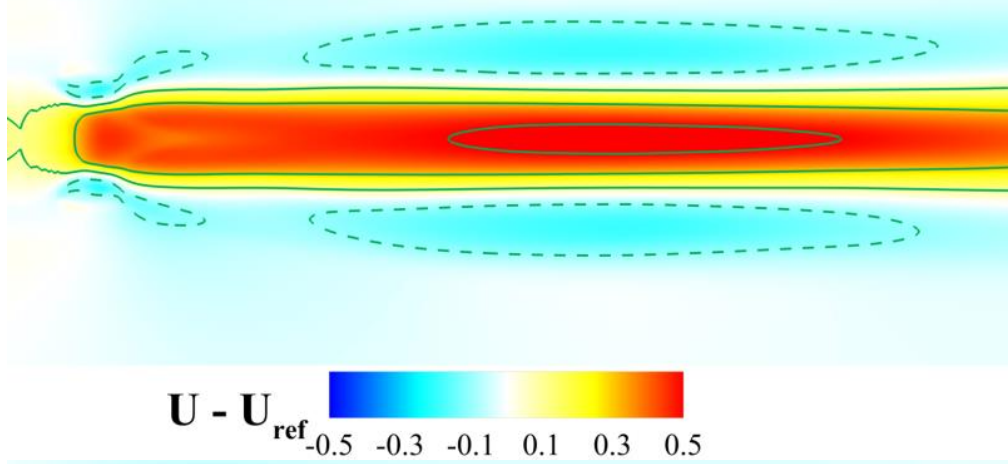

(c)
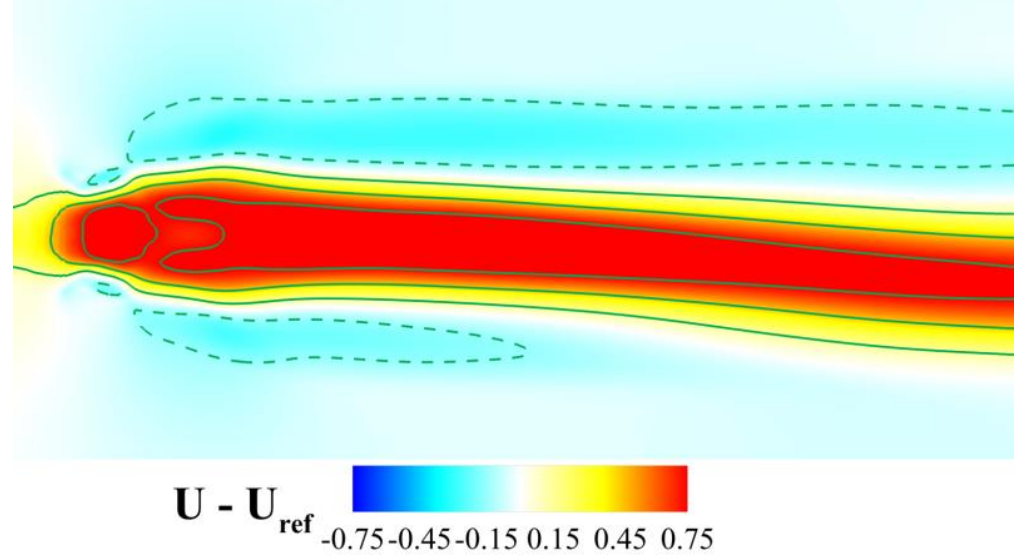

Figure 3-6. Mean flows of the cases (a) Opt $C_{T}$, (b) Opt $\eta$, and (c) Rigid. The incoming flow velocity is subtracted from the mean flows. 
We further study the mean flows of these three cases, and the corresponding results are shown in Figure 3-6. Due to the different wake structures discussed above, the mean flows of the three cases show different characteristics. For the case Opt $C_{T}$, very strong mean flow, which corresponds to the largest thrust production of this case, can be observed. However, the mean flow width expands a small amount in the far wake region, which suggests lower propulsive efficiency. For the case Opt $\eta$, a weak mean flow with no wake expansion can be identified. This wake structure suggests a low thrust production but high propulsive efficiency of the wing. No wake deflections can be observed in the two optimum cases. For the case of Rigid, a moderate mean flow with obvious wake deflection can be observed, which suggests low propulsive performance. In summary, the flow modulation due to the dynamic camber formation could greatly improve the aerodynamic performance of the wing.

\subsection{Chapter Summaries}

A gradient-based optimization frame has been conducted to accomplish the optimal dynamic camber control of 2D flexible wing for maximizing the thrust production and propulsive efficiency. Direct Numerical Simulation (DNS) is used to gain unsteady flow around the flapping wing and its aerodynamic performance. Wing morphological parameters $\left(P_{R}, \beta_{m}, \varphi\right)$ are defined and two optimum sets (case Opt $C_{T}$ and case Opt $\eta$ ) of those parameters are found. Results show that the thrust production of the case Opt $C_{T}$ is increased by $29.1 \%$, and the propulsive efficiency of the case Opt $\eta$ is increased by $36.2 \%$ comparing to a case of a completely rigid flapping wing (case Rigid). The wake structures 
of these cases show different characteristics. Typical $2 \mathrm{~S}$ and $2 \mathrm{P}$ wake structures are identified in the case Opt $C_{T}$ and case Opt $\eta$, respectively. For the case Rigid, a $2 \mathrm{~S}$ wake structure with asymmetric property can be observed, which corresponds to obvious deflection of the mean flow. The flow modulation due to the dynamic camber formation could greatly improve the aerodynamic performance of the wing. 


\section{Computational Optimization of Dynamic Twisting of}

\section{Pitching-rolling Plates on Aerodynamic Performance}

Natural swimmers/flyers are all equipped with deformable fins/wings, which can actively or passively produce deformations in both spanwise and chordwise [105-108]. It is reported that such deformations can provide extra hydrodynamic/aerodynamic benefits, including the increment in force production and the decrement in energy consumption [109-111]. The flexible propulsors are of great interest to both scientific study [112-114] and the engineering design [115-117].

Flapping motion is widely adopted in the biological propulsion system, such as fish pectoral fins and insect/bird wings. A lot of previous studies on the flapping motion are based on a simplified kinematic model of pitching-plunging [118-120], which assumes uniform motions in spanwise direction. However, a real fin/wing in nature presents pitching-rolling motion, in which the plunging motion is replaced by a rolling of the fin/wing about its root. The presence of rolling motion will enlarge the threedimensionality of the problem, which may be important to the flow features or the performance of the propulsors. Several experimental works are performed previously to study the performance of pitching-rolling plates with different shapes $[121,122]$. They found that twisting plays an important role in the performance of the pitching-rolling plate.

In this work, our purpose is to explore the effects of both spanwise and chordwise morphing in a flapping propulsor. A deformable plate model in pitching-rolling motion, which is to mimic the propulsor flapping around its root, is built. The performance and wake structure related to the deformable motion are discussed in detail. The paper will be 
organized as follows. The methodology applied by this work will be introduced first. Next, we will present results of rigid pitching-rolling plates with different pitching amplitudes. After that, the plate surface morphing effects will be discussed. At last, the conclusion of this work will be presented.

\subsection{Problem Definition}

The rigid plate kinematics, which are the base kinematics of this work, are governed by the following equations:

$$
\left\{\begin{array}{l}
\phi(t)=-\frac{1}{2} \phi_{\mathrm{m}} \cos (2 \pi f t) \\
\alpha(t)=\alpha_{m} \sin (2 \pi f t)
\end{array}\right.
$$

where $\phi$ is the rotating angle and $\alpha$ is the plate pitching angle. $\phi_{m}$ and $\alpha_{m}$ are the corresponding amplitudes of these two angles. In this work, $\phi_{m}$ is chosen to be $90^{\circ}$ and $\alpha_{m}$ varies between $15^{\circ}$ and $60^{\circ}$. Figure 1 shows the schematic of this rigid plate kinematics. The plate's aspect ratio applied in this work is $A R=2$.

Plate deformation patterns, which are twisting and bending, are modeled in both time and space. For twisting, as shown in the following equations, Fourier series with $N=2$ are used in the temporal modeling:

$$
\zeta(t)=\frac{a_{0}}{2}+\sum_{n=1}^{N} a_{n} \cos \left(\frac{2 \pi n t}{T}\right)+b_{n} \sin \left(\frac{2 \pi n t}{T}\right)
$$


where $\zeta$ is twisting index. $T$ is the flapping period. $a_{i}$ and $b_{i}$ are the corresponding Fourier coefficients, which are determined via solving the following system of equations:

$$
\zeta(0)=0 ; \zeta\left(t_{1}\right)=\zeta_{m}^{D} ; \zeta\left(t_{2}\right)=\zeta_{m}^{U} ; \zeta^{\prime}\left(t_{1}\right)=0 ; \zeta^{\prime}\left(t_{2}\right)=0
$$

where $t_{1}$ and $t_{2}$ denote the timings of maximum twisting in downstroke and upstroke respectively. $\zeta_{m}^{D}$ and $\zeta_{m}^{U}$ denote twisting index amplitudes in downstroke and upstroke respectively, which are control parameters of twisting.

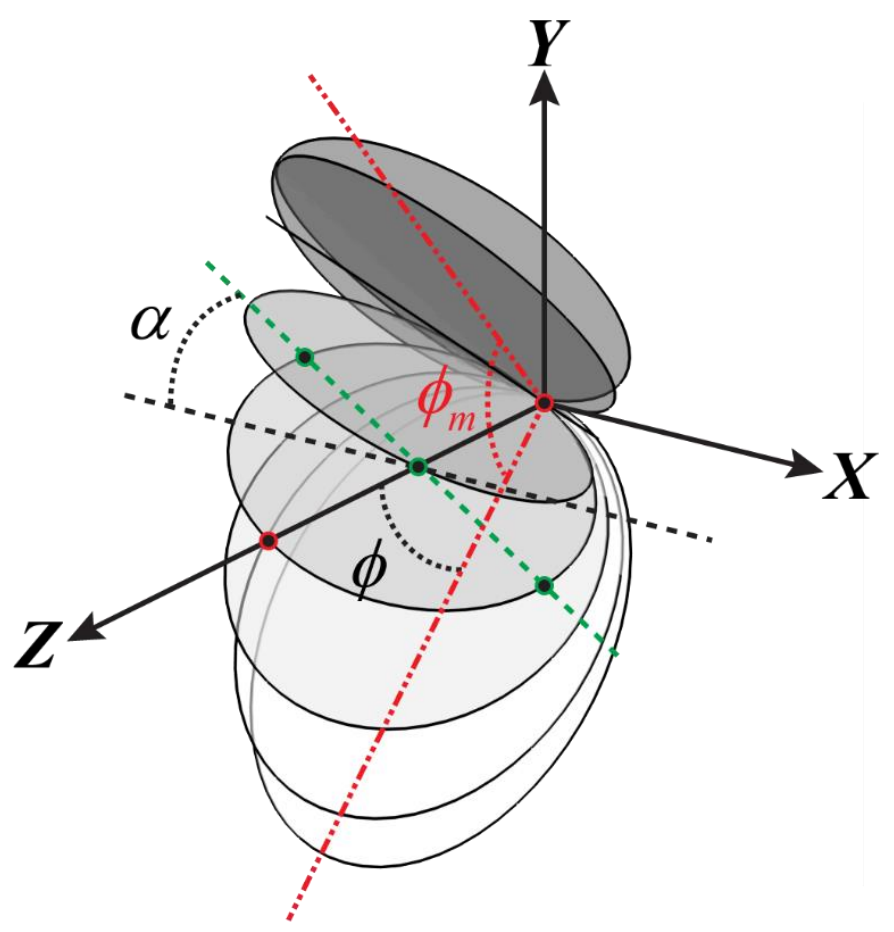

Figure 4-1. Schematic plot showing the definitions of plate kinematics.

For the spatial modeling of plate twisting, local twisting angles follow a linear distribution. It is based on observations of real insect wings/fish fins deformations through 
high-speed cameras [108]. Figure 2(a) shows the schematic plot of twisting modeling. The following equations show the spatial modeling of wing twisting:

$$
\chi_{s}=\frac{s \zeta}{100}
$$

where $s$ denotes the plate span location $(0<s<100)$.

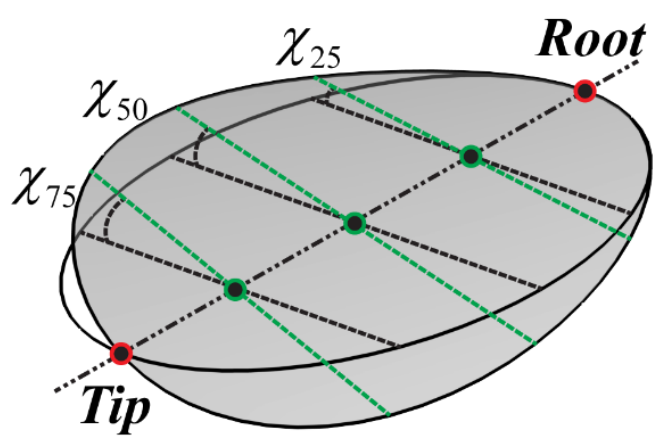

Figure 4-2. Schematic plot showing the modeling of plate twisting.

For all cases discussed in the results section, the twisting index and inflexion angle amplitudes in downstroke and upstroke are set equal with each other, in order to reduce the parameters involved in the parametric study. In summary, we have two parameters $\left(\zeta_{m}\right.$ and $\varphi$ ) to control the deformations of the pitching-rotating plates. We will discuss 4 cases in the following sections, which are rigid plate cases with different pitching amplitudes. Based on the results of those 4 cases, we choose one of them to be the baseline case and find optimal configurations of plate surface morphological parameters on the aerodynamic performance based on this case. 


\subsection{Computational Setup}

Figure 3 shows the stretching grids configuration of this work. The computational domain has the dimension of $20 \times 20 \times 20$, and the dense region has the dimension of $8.0 \times 6.0 \times 2.2$. The number of grids used in the dense region are $296 \times 224 \times 72$. Along the plate chord, there are no less than 35 grids are used to resolve the moving boundary. The non-dimensional incoming velocity $U_{\infty}$ is fixed as 1 , and zero gradient boundary condition is applied to the downstream boundary. No-slip boundary condition is applied at the plate surface. As defined in the following equations, the Reynolds number and Strouhal number of all cases discussed below is fixed as 200 and 0.6 , respectively.

$$
\operatorname{Re}=\frac{U_{\infty} c}{v}, \quad S t=\frac{f A}{U_{\infty}}
$$

Where $c$ is mid-chord length, $v$ denotes kinematic viscosity, $f$ is flapping frequency and $A$ stands for peak-to-peak amplitude measured at the mid chord.

Table 4-1. Pitching-rolling plate cases without surface morphing.

\begin{tabular}{lcc}
\hline \hline Cases & $\phi_{m}$ & $\alpha_{m}$ \\
\hline 1 & $90^{\circ}$ & $15^{\circ}$ \\
2 & $90^{\circ}$ & $30^{\circ}$ \\
3 & $90^{\circ}$ & $45^{\circ}$ \\
4 & $90^{\circ}$ & $60^{\circ}$ \\
\hline
\end{tabular}




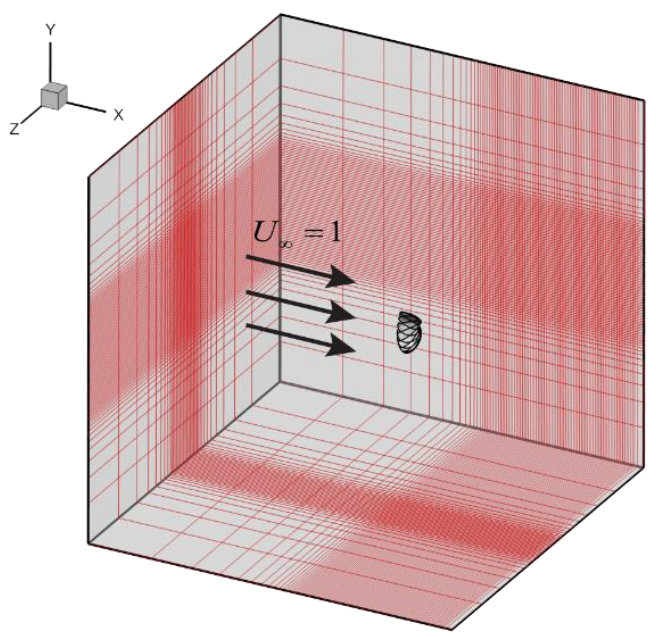

Figure 4-3. Stretching grids configuration used in the current work.

\subsection{Pitching-rolling Plate without Surface Morphing}

In this section, we first present the results of rigid pitching-rolling plates with different pitching amplitudes. And then, the effects of chordwise twisting and spanwise bending, which are added to a baseline rigid pitching-rolling plate with pitching amplitude equals to $30^{\circ}$, will be examined. Finally, the mechanism for the efficiency improvement of twisting will be discussed. We use thrust coefficient $\left(C_{T}\right)$ and power coefficient $\left(C_{P W}\right)$ to quantify the performance of the pitching-rolling plate, which are defined as,

$$
C_{T}=\frac{F_{x}}{0.5 \rho U_{\infty}^{2} s}, \quad C_{P W}=\frac{P_{o u t}}{0.5 \rho U_{\infty}^{3} s}
$$

Where $F_{x}$ is the thrust force; $P_{\text {out }}$ is the power output; $\rho$ is the density of the fluid and $S$ is the area of the plate. The time averaged $C_{T}$ and $C_{P W}$ over one flap cycle during the 
steady state are denoted by $\overline{C_{T}}$ and $\overline{C_{P W}}$ respectively. The propulsive efficiency is quantified by $\overline{C_{T}} / \overline{C_{P W}}$ in this paper.

Figure 4-4 shows the time variation of the thrust and power coefficients for various pitching amplitudes with $S t=0.6$ and $\mathrm{Re}=200$. The plots show the fifth cycle in the simulations, by which time the flow has reached a stationary state. For all cases except for the case with $\alpha_{m}=60^{\circ}$, the thrust peaks twice in each cycle at the time instant in the cycle when the foil is near the center of its trajectory, and so as the power coefficients peaks. In the $\alpha_{m}=60^{\circ}$ case, double peaks of thrust and power coefficients appears in each half stoke, which may be the results of wake capture in higher pitching amplitude cases, while lower pitching amplitude cases cannot interact with the wake of previously shed vortices due to limited motions.
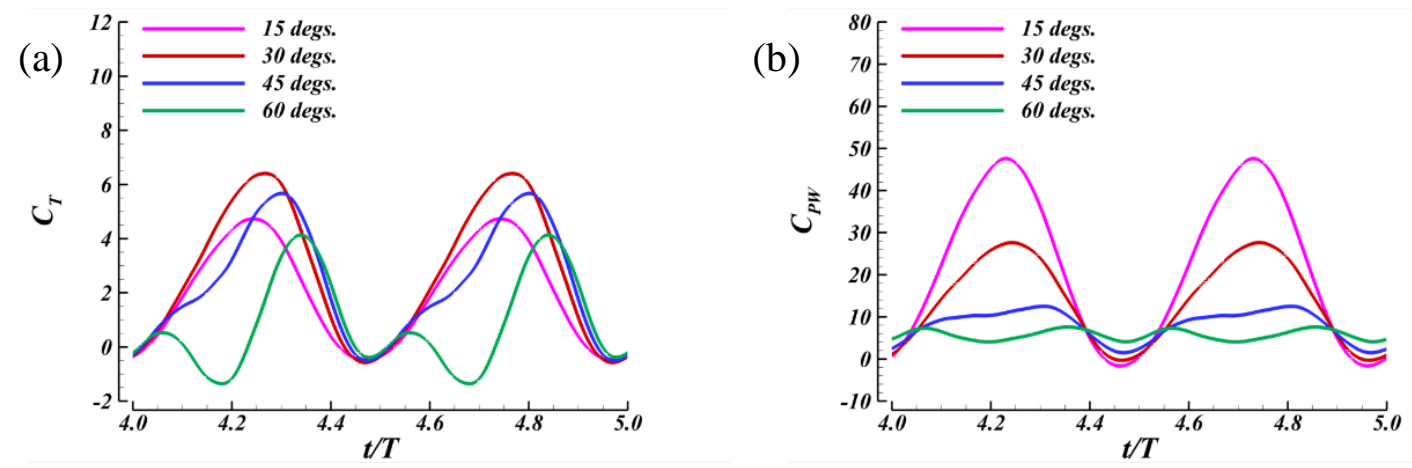

Figure 4-4. Thrust (a) and power (b) coefficient of the pitching-rolling plate with different pitching amplitudes (from $15^{\circ}$ to $60^{\circ}$ ). 
Table 4-2. Time averaged value of $C_{T}, C_{P W}$, and $C_{T} / C_{P W}$ of rigid pitching-rolling plate during the fifth flapping cycle.

\begin{tabular}{lcccc}
\hline \hline$\alpha_{m}$ & $15^{\circ}$ & $30^{\circ}$ & $45^{\circ}$ & $60^{\circ}$ \\
\hline$\overline{C_{T}}$ & 1.98 & 2.84 & 2.34 & 0.86 \\
$\overline{C_{P W}}$ & 21.37 & 14.10 & 7.86 & 5.45 \\
$\overline{C_{T}} / \overline{C_{P W}}$ & 0.093 & 0.202 & 0.298 & 0.158 \\
\hline
\end{tabular}

Table 4-2 listed cycle averaged values of thrust and power coefficients, and also the propulsive efficiencies, which defined as thrust over power ratio. It is found that the case with $\alpha_{m}=30^{\circ}$ has the highest thrust production while the propulsive efficiency is relatively high among the 4 cases. Previous study [121] on a pitching-rolling plate at high Reynolds number also found that the pitching amplitude variation can change the propulsive performance in terms of both the thrust and the propulsive efficiency.

Figure 4-5 shows the flow features of the case with $\alpha_{m}=30^{\circ}$. A thrust producing wake can be identified in Figure 4-5(a). Vortex pairs with different directions, or say, vortex rings, can be observed, which will induce local jets (red arrow) to the direction opposite to the thrust direction. Figure 4-5(b) shows vortex structures in three-dimensional space. The shed trailing edge vortex (TEV), along with the tip vortex (TV), connect with the newly developing leading edge vortex (LEV) and root vortex (RV) to form a vortex ring, which will shed right after the stroke reversal. Those vortex rings will induce flow jets as shown in Figure 4-5(a). The flow structures of a typical pitching-rolling plate is similar to that of a pitching-plunging plate [118]. 
(a)

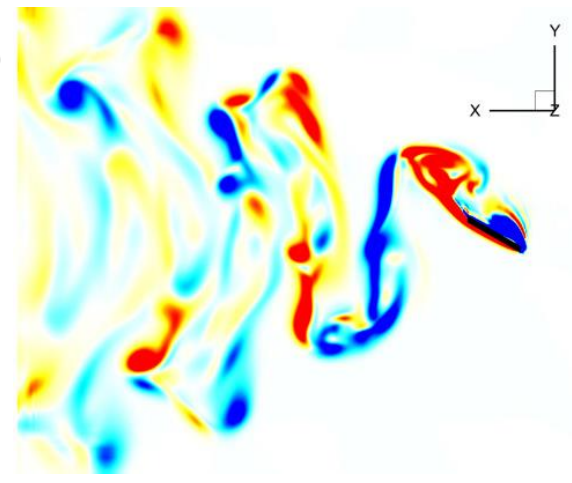

(b)

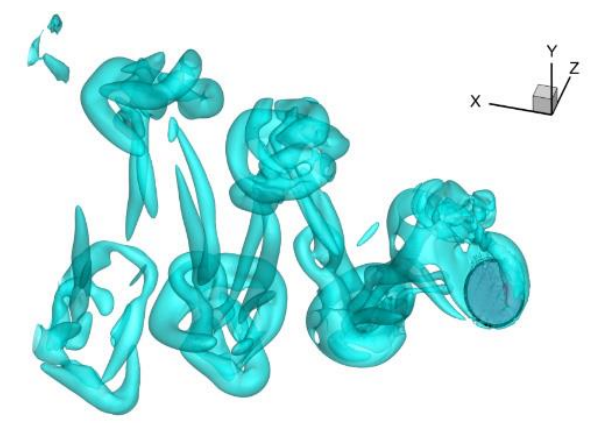

Figure 4-5. Flow structure of a rigid pitching-rolling plate (pitching amplitude is $30^{\circ}$ ) at mid-downstroke. (a) contour of $\omega_{z}$ on the vertical cut slice at 2/3 span away from the root; (b) perspective view of the 3D flow. The vortical structures are identified by the iso-surface of Q-criteria $(\mathrm{Q}=1.0)$.

\subsection{Pitching-rolling Plates with Optimal Surface Morphing}

In the previous sub-section, we compared the performance of rigid pitching-rolling plates with different pitching amplitude $\left(\alpha_{m}\right)$ and found that the case with $\alpha_{m}=30^{\circ}$ has the highest thrust. In this sub-section, we use this case as the baseline case, and investigate optimal configurations of dynamic twisting of the plates on aerodynamic performance. The corresponding objective functions are chosen as the cycle averaged thrust coefficient (case Opt $C_{T}$ ) and the propulsive efficiency (case Opt $\eta$ ). 

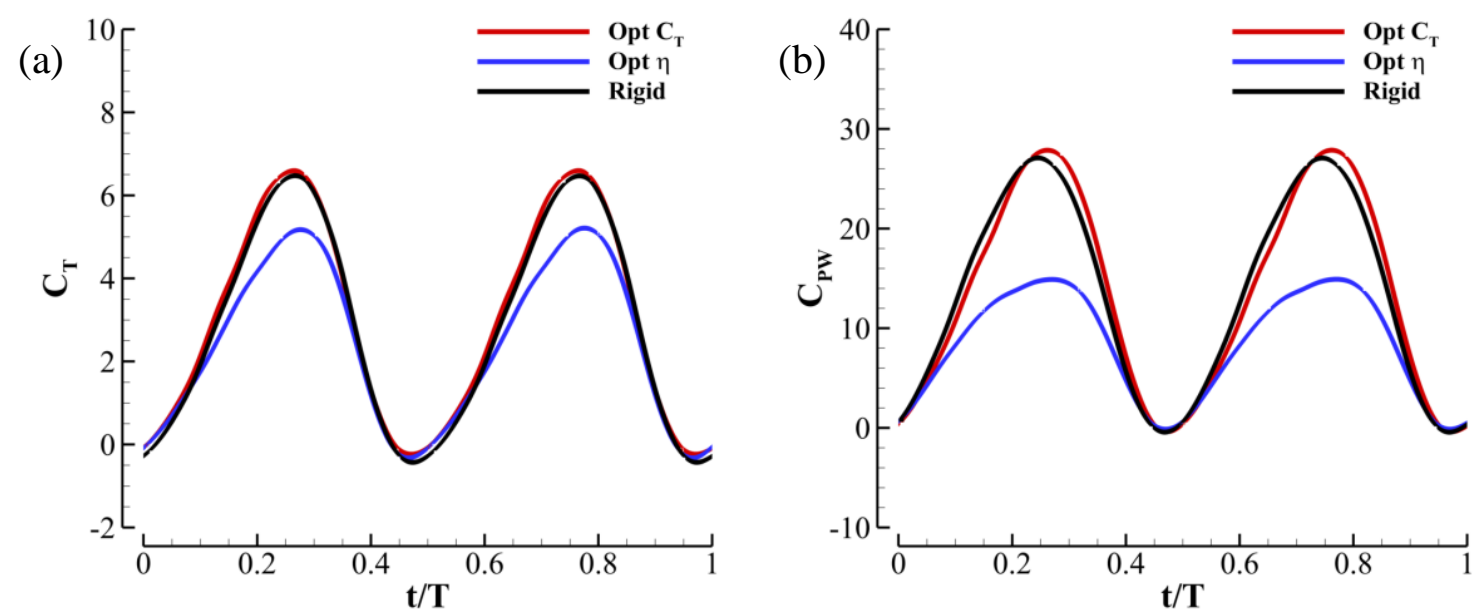

Figure 4-6. Thrust (a) and power (b) coefficient histories of the pitching-rolling plates with optimized dynamic twisting. Results of a completely rigid plate (case Rigid) are also shown for comparison.

Figure 4-6 and Table 4-3 shows the performance comparison of the two optimization cases and case Rigid. First, we compare the results of the case Opt $C_{T}$ to the case Rigid. According to Table 4-3, it is found that the thrust increment is $6 \%$ and the efficiency increment is $8.9 \%$. This result demonstrates that the effect of dynamic twisting on the thrust production is relatively small since the choice of the plate pitching amplitude $\left(30^{\circ}\right)$ is already in an optimal region according to the parametric studies discussed in previous section.

The effect of dynamic twisting on propulsive efficiency is more sensitive. The results of case Opt $\eta$ show that the propulsive efficiency improved by $43.1 \%$ comparing to that of the case Rigid, while the corresponding thrust production only drops by $14.8 \%$. This 
results demonstrate that dynamic twisting is critical for the propulsive efficiency of the pitching-rolling plates.

Table 4-3. Time averaged value of $C_{T}, C_{P W}$, and $C_{T} / C_{P W}$ of rigid pitching-rolling plate with optimized dynamic twisting. Results of a completely rigid plate (case Rigid) are also shown for comparison.

\begin{tabular}{lccc}
\hline \hline & Opt $C_{T}$ & Opt $\eta$ & Rigid \\
\hline$\overline{C_{T}}$ & 3.02 & 2.42 & 2.84 \\
$\overline{C_{P W}}$ & 13.7 & 8.38 & 14.10 \\
$\overline{C_{T}} / \overline{C_{P W}}$ & 0.220 & 0.289 & 0.202 \\
\hline
\end{tabular}

The performance of the plate is directly related to the induced flow structures. Here, we compare the flow features of the two optimization cases (Opt $C_{T}$ and Opt $\eta$ ). As shown in Figure 4-7, it is found that the surface morphing of the plate do not change the main flow features of pitching-rolling plates. Two sets of inclined vortex ring loops can be observed in both cases. The mean flows of both cases show the same feature, i.e., two hornlike backward jets is induced by the vortex rings in the downstream wake. However, the difference is also obvious. The inclination angles ( $\beta$ in Figure 4-7b) of the wake in these two cases are quite different. The value of $\beta$ in case Opt $\eta$ is only $12.5^{\circ}$ which is only $35 \%$ of that in case Opt $C_{T}$. This leads to more concentrated backward jets in the case Opt 
$\eta$ than that in the case Opt $C_{T}$, which is mainly responsible for the propulsive efficiency improvement.

(a)

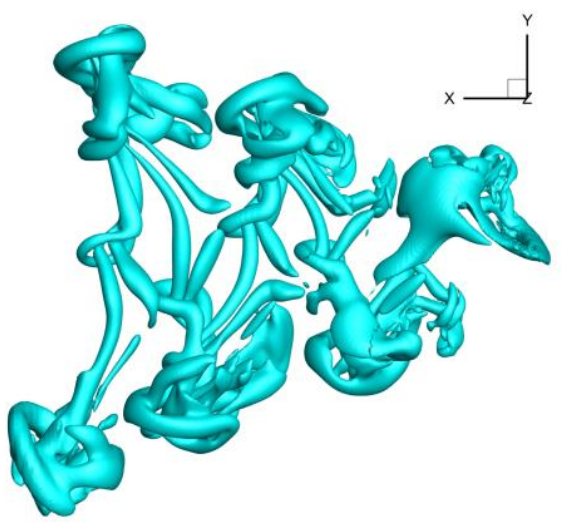

(c)

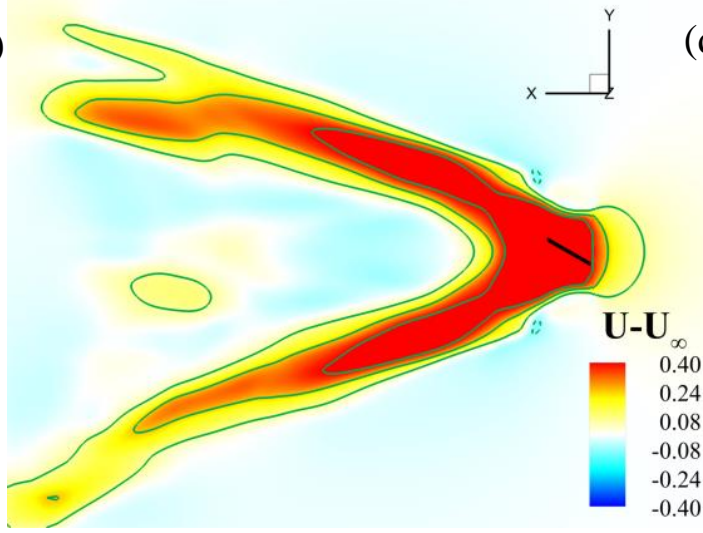

(b)

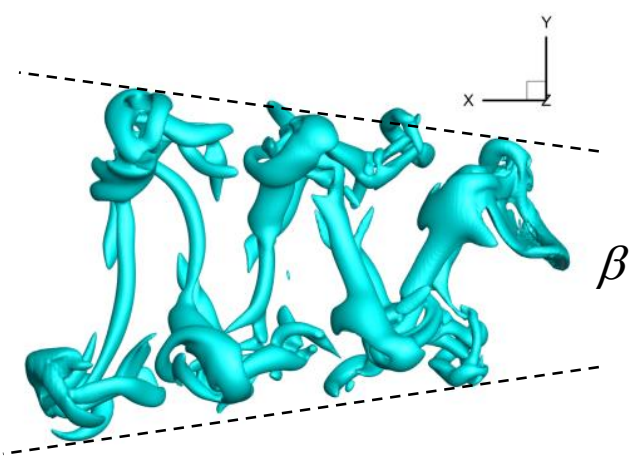

(d)

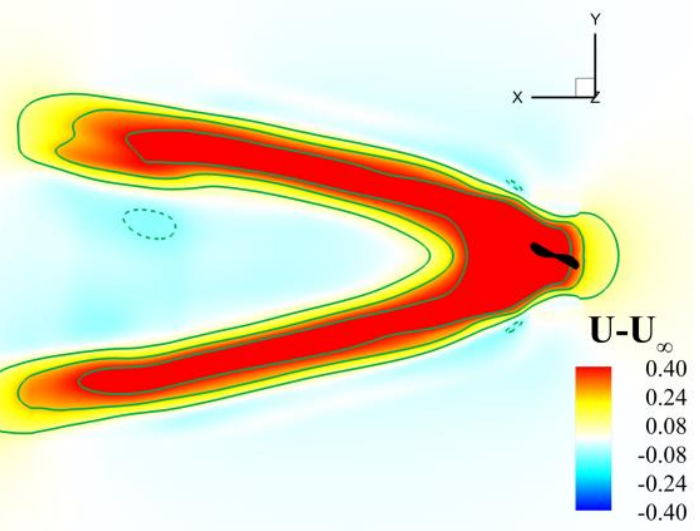

Figure 4-7. Side view of the flow structures of (a) case Opt $C_{T}$ and (b) Opt $\eta$. Mean flow (incoming flow subtracted) contour of (c) case Opt $C_{T}$ and (d) Opt $\eta$.

\subsection{Chapter Summaries}

In this work, computational optimization of dynamic twisting of pitching-rolling plates on aerodynamic performance is performed. First of all, we build a morphing plate model, which includes dynamic chordwise twisting in pitching-rolling motion inspired from 
biological propulsors. Thrust production, power consumption and flow structures of the model plates are obtained through an in-house immersed boundary method based CFD solver. Rigid pitching-rolling plates with different pitching amplitudes, varied from $15^{\circ}$ to $60^{\circ}$, are studied. The results show that the case with pitching amplitude of $30^{\circ}$ generates the highest thrust. Using this case as the baseline, optimal configurations of dynamic twisting for maximizing the thrust production and propulsive efficiency are found. The results show that the thrust production and propulsive efficiency of optimized plates can be improved by $6 \%$ and $43.1 \%$, respectively. Observations on the unsteady flow field of optimized cases show that the performance enhancements correspond to improved strength and attachment of leading-edge vortex. 


\section{Aerodynamics of Low Dimensional Models and Computational Optimization of a Hovering Dragonfly \\ Wing}

In previous chapters, we have used two different approaches to study the aero/hydrodynamics of flapping propulsors. We first perform computational optimization on canonical model problems, including a 2D pitching-plunging morphing plate and a 3D pitching-rolling morphing plate. After that, a physics-based methodology is used to study the wing aerodynamics of a real maneuvering hummingbird. In this chapter, the two approaches are combined together to study the wing aerodynamics of a hovering dragonfly.

\subsection{Data Acquisition and Computational Setup}

As introduced in section 2.1.3, the motion of a hovering dragonfly is reconstructed using the high-speed photogrammetry and the joint-based reconstruction technique. We focus on the forewing motion and its associated aerodynamics in this section. The spherical-coordinates-based singular value decomposition (SSVD) method is used to decompose the complex forewing motion. As shown in Figure 2-7, the first three SSVD modes contain $96.0 \%$ of the original motion. Actually, only the first two dominant modes contain $93.1 \%$ of the original motion and their motions are shown in Figure 2-8(b) and (c). Low dimensional models are then reconstructed based on the first three SSVD modes, and numerical simulations regarding those models are conducted to study their associated aerodynamic performance and wake structures in the following sections. 
Figure 5-1 shows the constructed realistic full-body model immersed in the threedimensional non-uniform Cartesian grid. $\mathrm{Y}$ is the vertical direction with $+\mathrm{Y}$ pointing upward. The wings in this study are modeled as deforming membranes, and the body is also deformable and both of them are controlled by virtual joints.

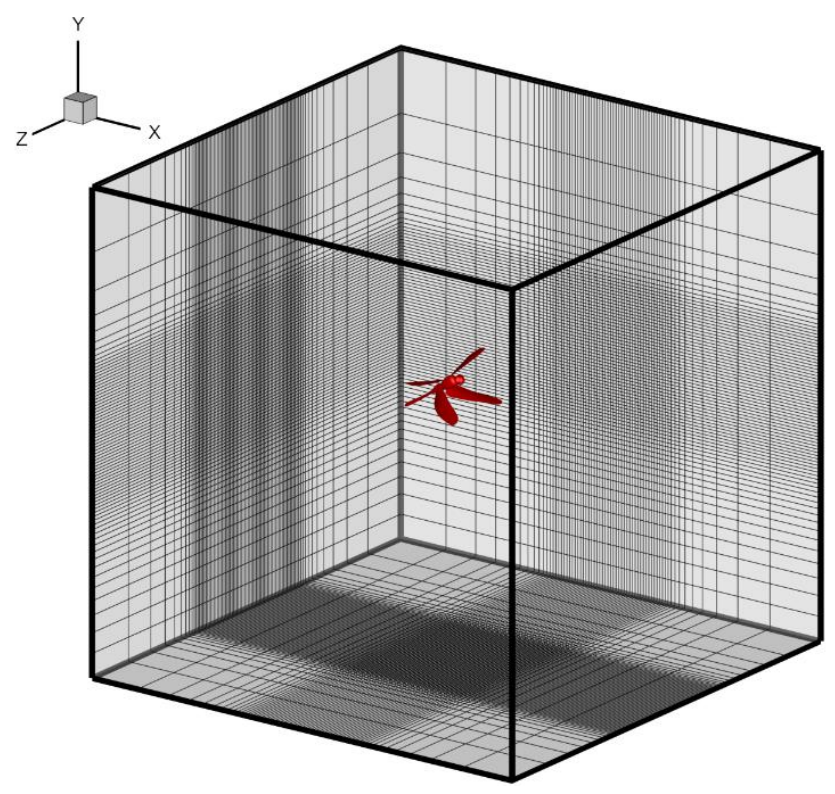

Figure 5-1. The constructed realistic wing-body model immersed in the three-dimensional non-uniform Cartesian grid.

The boundary conditions applied in this study is described as follows. On all of the boundaries, gradients of all velocity components are set to zero. The final domain size normalized by the mean chord length $c$ is $20 \times 20 \times 20$ and this large domain ensures that the boundaries do not have any significant effect on the computed results. As shown in Figure 5-1, the grid is designed to provide high resolution in the region around the body as well as the wake that is expected to develop below the body and wings. This grid was 
developed in an iterative fashion starting from a sequence of coarser grids. Computed results on these earlier meshes were examined, and the resolution in selected regions increased until the key features such as forces and vortex structures became essentially independent of the grid. The final grid adopted here has $288 \times 196 \times 176$ points. The grid size in all three directions are around 0.045 chord length, corresponds to 71 grids across the span and 22 across the chord-wise direction on the wing surface.

Three additional simulations on different grids were carried out to assess the convergence of the computed flow. Simulation 1 was carried out on a grid that had $25 \%$ more grid points than the baseline grid and simulation 2 was carried out on a grid with $25 \%$ fewer grid points than the baseline grid. Both of these simulations produced a maximum of $1 \%$ difference from the baseline in the mean lift and root-mean-square (R.M.S.) values of lift and drag. Finally, simulation 3 was carried out on a grid where the number of grid points in each direction around the wing were twice those in simulation 2 . This simulation also produces mean lift and R.M.S. lift and drag that are within $1 \%$ of simulation 2 and taken together with the other simulations, provide clear proof of the grid convergence of the current results.

\subsection{Model Dimensionality Effects}

The aerodynamics of low dimensional models for the forewing of a hovering dragonfly is investigated in this section. Simulations have been carried out using the precise wing kinematics extracted from the experiments (this case is termed here as mode-all). The nominal conditions for the current simulation correspond to a wing Reynolds number of 
about 1840 and a wing reduced frequency of 0.25 , and it matches the real conditions of the hovering flight. Although the main focus of the current work is the analysis of the aerodynamic and performance of low-dimensional models based on SSVD modes, some key results for the mode-all case are also included here, since comparison with the modeall case is key to understanding the scaling of the aerodynamic performance as the dimensionality of the models is increased. All the results presented here have been obtained by simulating the flow over four wing strokes. In computing mean quantities, we have discarded the first stroke, and all plots of instantaneous quantities correspond to the third cycle in the stroke by which time the flow has reached a well-established stationary state.

In the current study, we focus on the following low-dimensional models: mode 1, mode $1+2$, and mode $1+2+3$. All these gaits are studied at a Reynolds number of 1840 and a reduced frequency of 0.25 . Thus, the dynamical similarity between the mode-all case and the low-dimensional models is maintained, and this allows us to isolate the effect of model dimensionality on the wing performance.

\subsubsection{Effect of Model Dimensionality on Aerodynamic Performances}

In this section, we describe the effects of increasing the dimensionality of the forewing motion of the hovering dragonfly on the aerodynamic performances. The effects of model dimensionality on the quantitative characteristics of the wing are investigated, including force production and lift economy.

The time variations of lift, drag and power coefficients are presented for all the lowdimensional gaits and compared to the mode-all case in Figure 5-2(a - c), respectively. 
The force and power coefficient for a generic force $F$ and aerodynamic power $P$ are defined as:

$$
C_{F}=\frac{F}{1 / 2 \rho U_{r e f}^{2} A} \quad, \quad C_{P W}=\frac{P}{1 / 2 \rho U_{r e f}^{3} A}
$$

where $A$ is the wing area; $\rho$ is the air density; and $U_{r e f}$ is the reference velocity, which is chosen as the average velocity of the wing mid chord.

Several observations on how each SSVD mode contributes to the performances of the wing can be made from these results. It should be noted that only mode 1 can be simulated by itself. However, given the underlying nonlinearity of the flow, the contribution of mode 2 and mode 3 are investigated by considering the differences in the performances from the lower-level gait. Thus, the effect of mode 2 on performance is obtained by analyzing the differences between the performances of the mode 1 and mode $1+2$ cases. Similarly, the effect of mode 3 on wing performances can be assessed by comparing the performances of the mode $1+2+3$ case with that of the mode $1+2$ case.

As we can see in Figure 5-2 that, the aerodynamic performances are very similar for all cases except for the mode 1 only case. For the lift production, Figure 5-2(a) shows that all cases produce positive lift except for mode 1 case, which negative lift can be observed during the upstroke. Also, the lift production for mode 1 case during downstroke is much smaller (about 2.5 times smaller) than other cases. For the drag production, Figure 5-2(b) shows that the drag is much higher for mode 1 only case during the downstroke. For upstroke, the drag productions are very similar for all cases. These are because the lack of wing rotation about the span axis, which is included in the deformation mode (mode 2). 
During the downstroke, the wing angle of attack for mode 1 case is much greater than other cases, and it leads to less lift and more drag production (Figure 5-2b). During the upstroke, the wing angle of attack for mode 1 case is greater than 90 degrees due to lack of wing rotation about the span axis, and negative lift produces at this phase. The involvement of the deformation mode (mode 2) can greatly improve the lift production and reduce the drag produced by the wing.
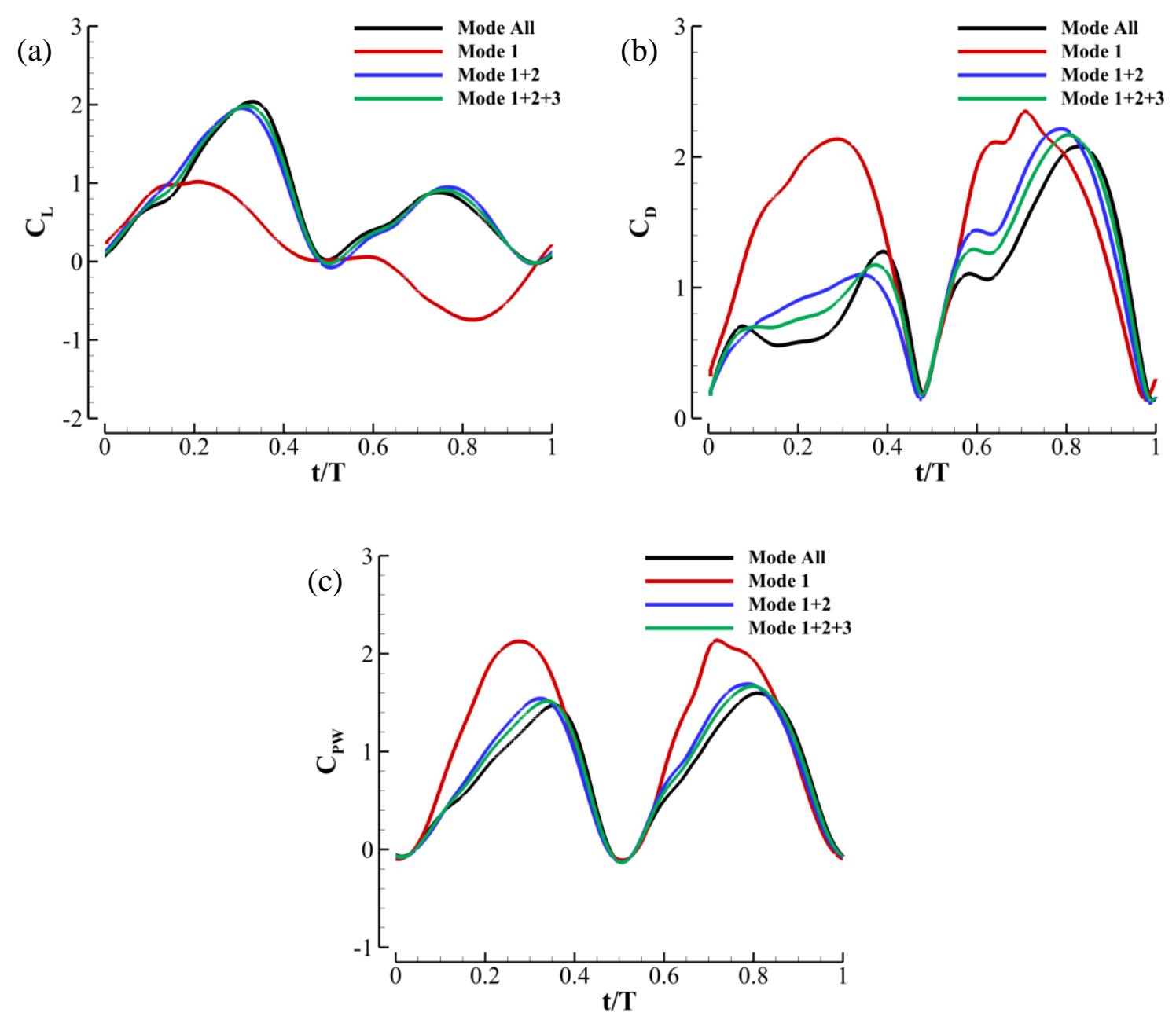

Figure 5-2. Comparison of the time course of aerodynamic performances between the Mode-all and low-dimensional gaits. (a) lift; (b) drag; (c) power. 
Table 5-1. Cycle averaged aerodynamic performance of the mode-all and lowdimensional gaits.

\begin{tabular}{lcccccc}
\hline & $\lambda^{*}(\%)$ & $\overline{C_{L}}$ & $\overline{C_{D}}$ & $\overline{C_{P W}}$ & $\eta$ & $\begin{array}{c}\text { Lift production } \\
(\%)\end{array}$ \\
\hline Mode-all & 100 & 0.752 & 1.021 & 0.776 & 0.969 & 100 \\
Mode 1 & 84.7 & 0.149 & 1.464 & 1.087 & 0.137 & 19.8 \\
Mode 1+2 & 93.1 & 0.749 & 1.100 & 0.818 & 0.916 & 99.6 \\
Mode 1+2+3 & 96.0 & 0.750 & 1.082 & 0.813 & 0.923 & 99.7 \\
\hline
\end{tabular}

For the aerodynamic power histories (Figure 5-2c), we can see that all cases present two peaks during the cycle, and the amplitude of power consumption for mode 1 case is much greater than other cases at both down and upstrokes. This suggests that including the deformation mode (mode 2) can also reduce the power consumption of the dragonfly.

Cycle averaged aerodynamic performances are listed in Table 5-1 for mode-all and low dimensional wing gaits, including the lift economy $\eta$ that is defined as $\eta=\overline{C_{L}} / \overline{C_{P W}}$. We can see from the table that mode $1+2$ is a good approximation of the original wing motions (Mode-all). It contains only two dominant SSVD modes, and the motion is recovered over $90 \%$. The associate aerodynamic performances of mode $1+2$ case are very similar to the mode-all case as. The lift production is recovered $99.6 \%$ of the original motion. 


\subsubsection{Effect of Model Dimensionality on Vortex Structure}

In this section, we describe the effect of increasing the dimensionality of the wing motion on the three-dimensional vortex structures. We first focus on the qualitative features of the flow for these low-dimensional gaits and subsequently address the effect of model dimensionality on the quantitative aerodynamic characteristics of the wing, including leading edge vortex circulation distribution and time history.
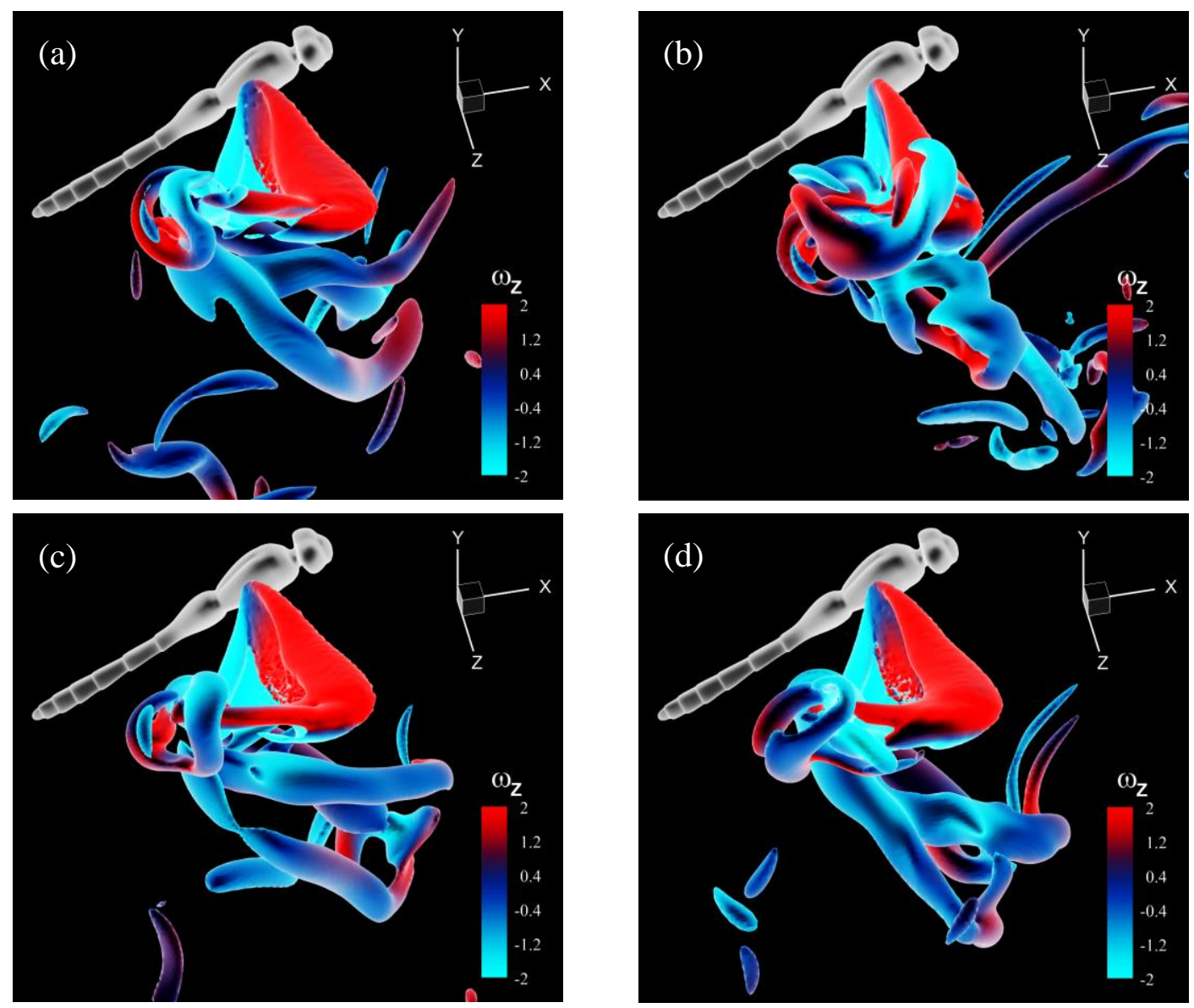

Figure 5-3. Wake structures for the (a) mode-all, (b) mode 1, (c) mode 1+2 and (d) mode $1+2+3$ cases. The associate time instance is $t / T=0.25$. 
Figure 5-3 shows the vortex structures at the mid downstroke $(t / T=0.25)$ for the modeall, mode 1 , mode $1+2$ and mode $1+2+3$ cases. The vortex structures are identified by plotting contours of the 'swirl strength' which is the magnitude of the imaginary part of the complex eigenvalue of the velocity deformation tensor $[123,124]$. In these figures, the dragonfly body is shown for visualization purposes only and is not included in the simulations. Figure 5-3(a) shows the vortex structures of the mode-all case. One of the most visible vortex structure at this time instance is the developing vortex ring that forms by connecting leading edge vortex, newly formed tip vortex, trailing edge vortex and root vortex. Also visible are the previously shed tip vortex and vortex ring just below the newly formed vortex ring. The previous shed vortex ring associated with the previous wing stroke has convected further downstream by this time. Far wake structures, which can be observed approximately one body length below the wing, are consist of many small vertices with slender shape due to the dissipation effect.

Figure 5-3(b) shows the vortex structures of the mode 1 only case. The most significant difference from the mode-all case is that the expected newly formed vortex ring cannot be identified. Instead, a very large previous shed tip vortex can be observed. Also, the previously shed vortex ring is not well connected. Far wake structures disappear due to relatively small downwash. For the wake structures of mode $1+2$ and mode $1+2+3$ cases (Figure 5-3c and d), all flow features that observed in mode-all cases can be identified. Differences are located in the far wake structures. The far wake structures of mode $1+2$ case are smaller than the mode-all and mode $1+2+3$ cases, and the latter two cases present similar flow structures in the far wake. 
Figure 5-4 shows the vortex structures at the end of the downstroke $(\mathrm{t} / \mathrm{T}=0.5)$ for the mode-all, mode 1 , mode $1+2$ and mode $1+2+3$ cases. Figure 5-4(a) shows the vortex structures of the mode-all case. Different from the time instance of $t / T=0.25$, a strong tip vortex that extends from the tip of the wing all the way into the wake over a distance that roughly equals to the wing length. The newly formed vortex ring that is identified in Figure 5-3(a) is well developed and ready to shed. The previously formed vortex ring merges to a vortex tube with one end connect to the newly formed vortex ring. The far wake presents similar slender structures as that of the time instance $\mathrm{t} / \mathrm{T}=0.25$.
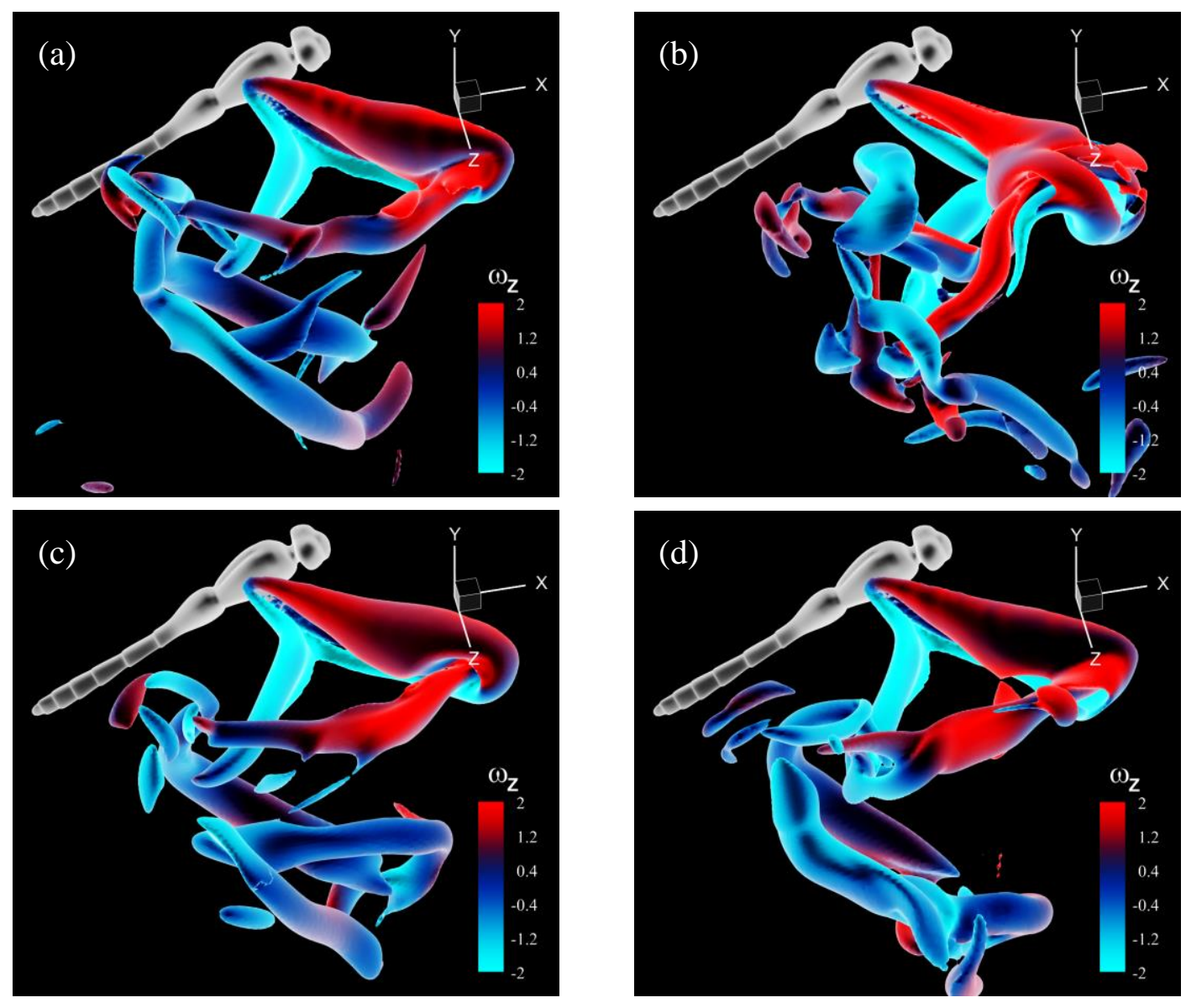

Figure 5-4. Wake structures for the (a) mode-all, (b) mode 1, (c) mode 1+2 and (d) mode $1+2+3$ cases. The associate time instance is $t / T=0.5$. 

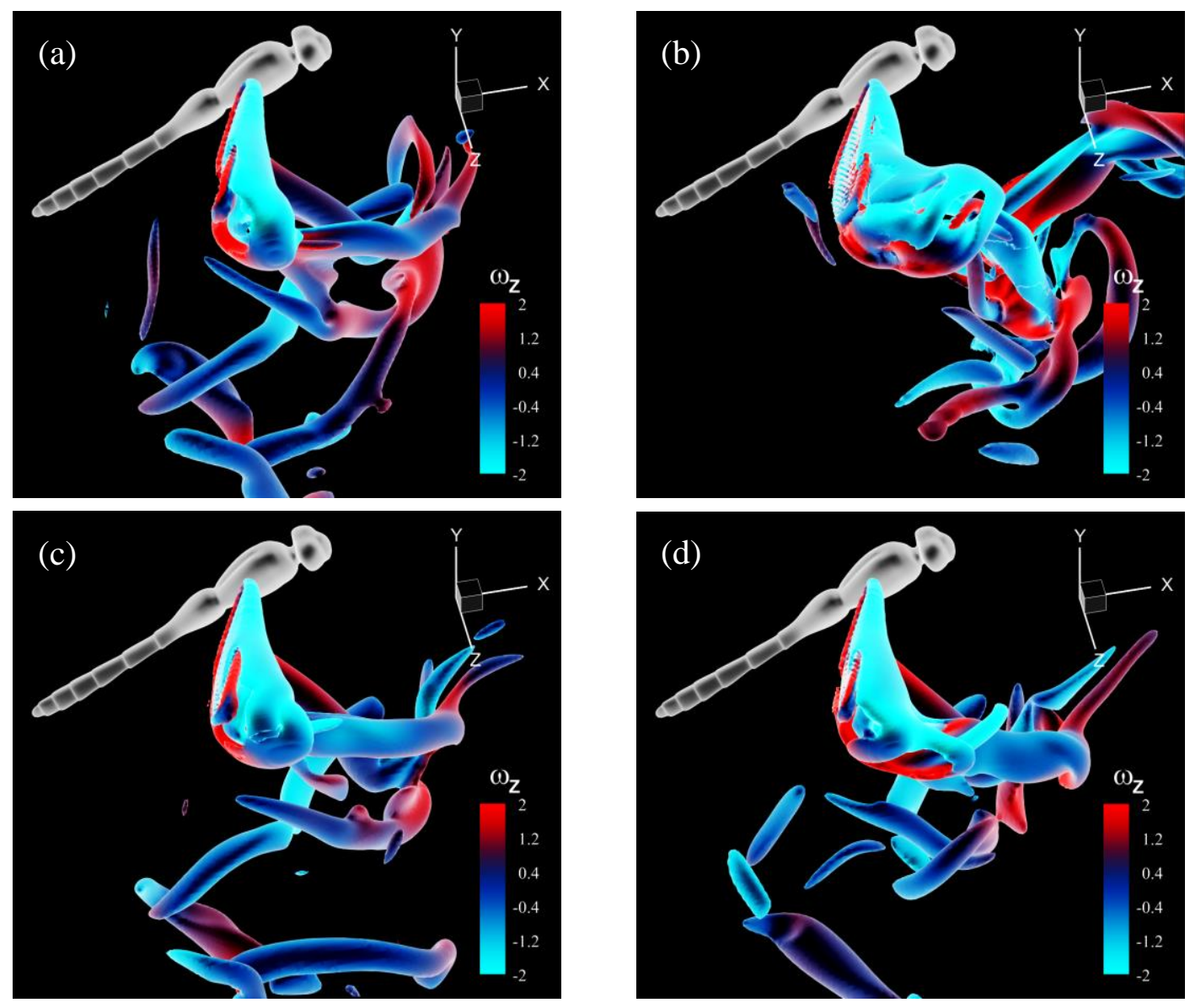

Figure 5-5. Wake structures for the (a) mode-all, (b) mode 1, (c) mode 1+2 and (d) mode $1+2+3$ cases. The associate time instance is $\mathrm{t} / \mathrm{T}=1.0$.

Figure 5-4(b) shows the vortex structures of the mode 1 only case. Different from the mode-all case, the tip vortex structures are much more complex and stronger. No vortex rings can be clearly identified, including both the newly formed and previously shed ones. Also, the far wake structures have disappeared. For the wake structures of mode $1+2$ and mode $1+2+3$ cases (Figure 5-4c and d), similar conclusions can be drawn, including 
similar newly formed vortex rings, and vortex tubes connect to vortex rings. However, the primary far wake structures of mode $1+2$ and mode $1+2+3$ cases are smaller to that of the mode-all case.

Figure 5-5 shows the vortex structures at the end of upstroke $(\mathrm{t} / \mathrm{T}=1.0)$ for the modeall, mode 1, mode 1+2 and mode 1+2+3 cases. Figure 5-5(a) shows the vortex structures of the mode-all case. Similar to the time instance of $t / T=0.5$, a strong tip vortex still can be observed. The previously shed vortex ring exists below the newly formed one, but not well connected. Far wake structures are similar to that of time instance $t / T=0.25$ and $t / T=0.5$. Figure 5-5(b) shows the vortex structures of the mode 1 only case. The most visible vortex structures in this case are the two strong vortex conglomerations, which are connected by complex newly formed tip vortex. Other than that, vortex rings cannot be observed. Instead, some small slender vortex structures can be observed aside from the lower vortex conglomeration. Similar to the other two time instances discussed above, no far wake structures can be identified. For the wake structures of mode $1+2$ and mode $1+2+3$ cases (Figure 5-5c and d), similar conclusions can be drawn.

In summary, by plotting three-dimensional vortex structures of the four cases (modeall, mode 1 , mode $1+2$ and mode $1+2+3)$ at three different time instances $(t / T=0.25,0.5$ and 1.0), we qualitatively study the effects of model dimensionality on vortex structures. We conclude that mode 1 case shows completely different vortex structures than other cases. Very strong tip vortex and vortex conglomerations can be observed in the wake while the vortex rings and far wake structures are not showing. For the other three cases, primary vortex structures are very similar. Small differences can be observed in the far wake. 

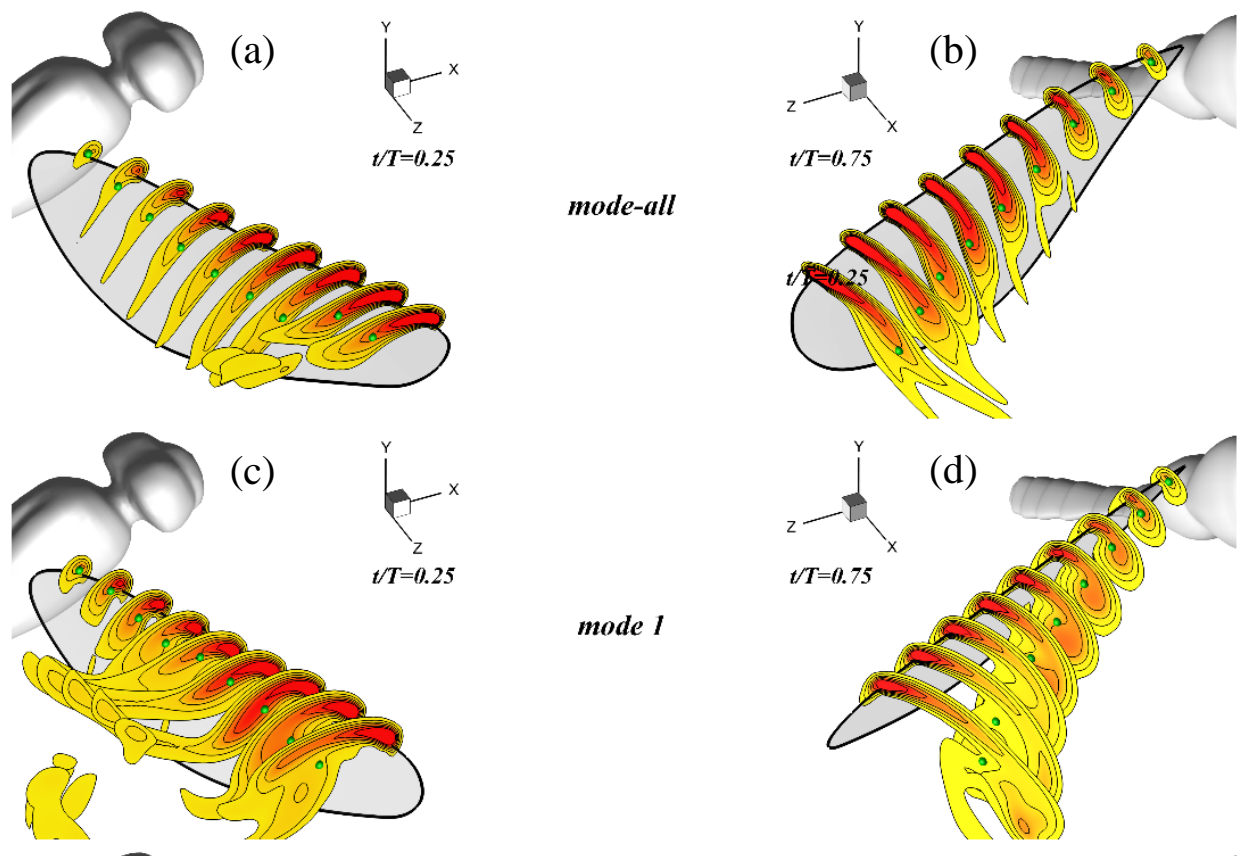

mode 1
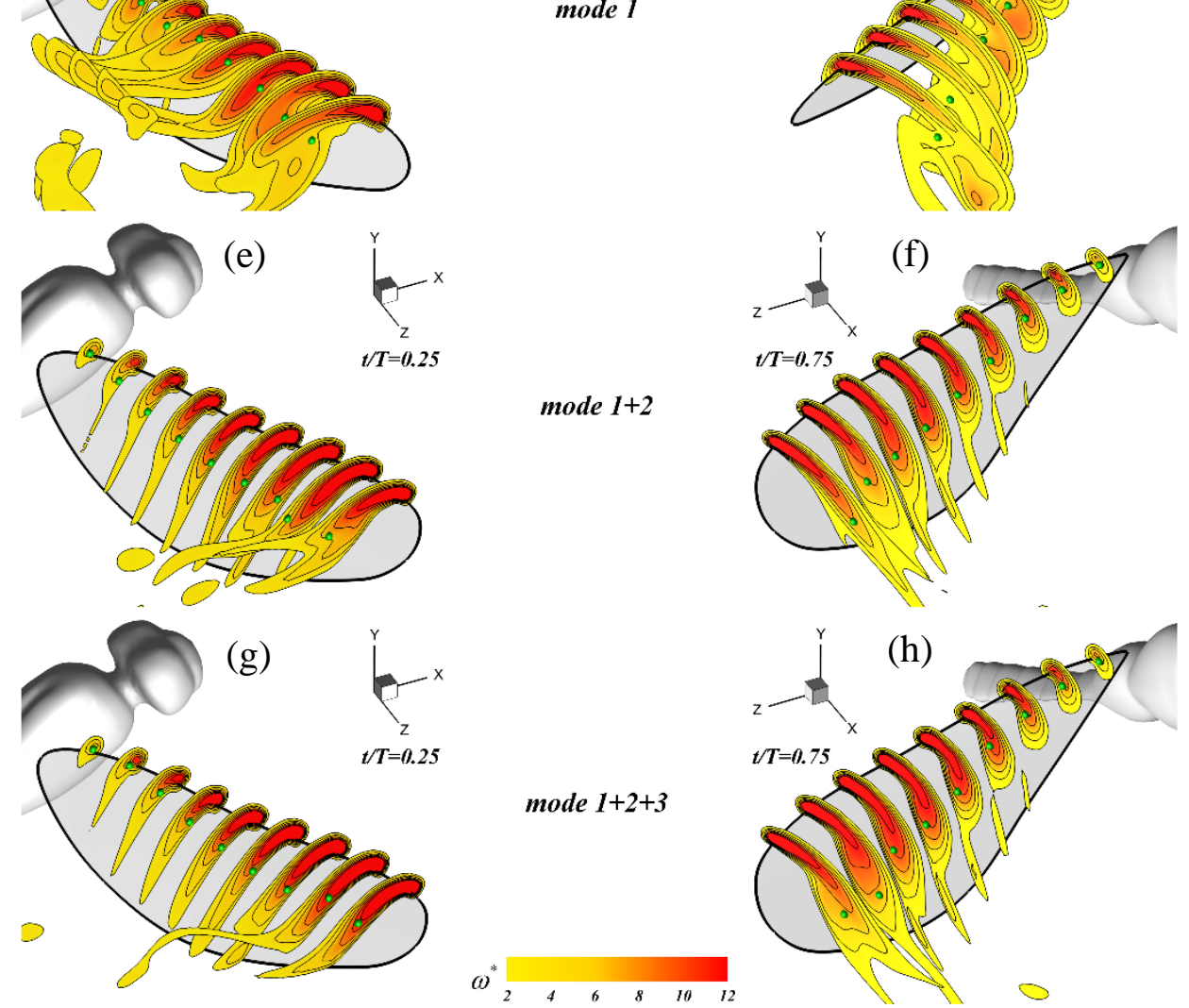

Figure 5-6. 2D slices of the leading edge vortex along the wingspan (10\% 90\%) at two time instances for $(\mathrm{a}, \mathrm{b})$ mode-all, $(\mathrm{c}, \mathrm{d})$ mode $1,(\mathrm{e}, \mathrm{f})$ mode $1+2$ and $(\mathrm{g}, \mathrm{h})$ mode $1+2+3$ cases. The corresponding vortex center are marked with green dots at each slice. The contours represent normalized span-wise vorticities. 
To further study the effects of dimensionality on the flow field, especially in the near wake, we cut slices along the wingspan to see the leading edge vortex structures and measure the associated circulations evaluated at each slice. Figure 5-6 shows the corresponding results. We can see from the figure that for all cases, the LEV shapes gradually grow bigger from the wing root to wing tip. More importantly, the corresponding vortex structures are very different in mode 1 only case comparing to other cases. The LEV shapes are much bigger, and the associated attachment is bad in mode 1 case. For other cases, the LEV shapes are similar. Small differences can be observed near the wing tip region. At mid downstroke $(\mathrm{t} / \mathrm{T}=0.25)$, the $\mathrm{LEV}$ attachment is pretty good for all cases except for the mode 1 only case. However, at mid upstroke ( $t / T=0.75)$, the LEV attachment is not as good as that at mid downstroke.

Quantitative measurements related to the LEV attachments of all cases discussed above are conducted. We first determine the LEV centers based on vortex shapes shown in Figure 5-6. After that, we measure the distances, which are named as lift-off distances, between LEV centers (green dots) and the wing surface to evaluate the LEV attachments. Figure 5-7 shows the results at mid downstroke $(\mathrm{t} / \mathrm{T}=0.25)$ and $\mathrm{mid}$ upstroke $(\mathrm{t} / \mathrm{T}=0.75)$. For the mode 1 only case, the lift-off distances are much higher than other cases in both time instances, which indicates bad LEV attachment. In addition, the ranges of the lift-off distance are much wider in mode 1 only case for both time instances. It ranges from 0.15 chords to 0.55 chords at mid downstroke, and from 0.20 chords to 1.09 chords at mid upstroke. Moreover, at mid downstroke, a small peak of lift-off distance shows up at $60 \%$ span and then drops at $70 \%$ span, which corresponds to the shed of LEV at $70 \%$ span in Figure 5-6(c). 
(a)

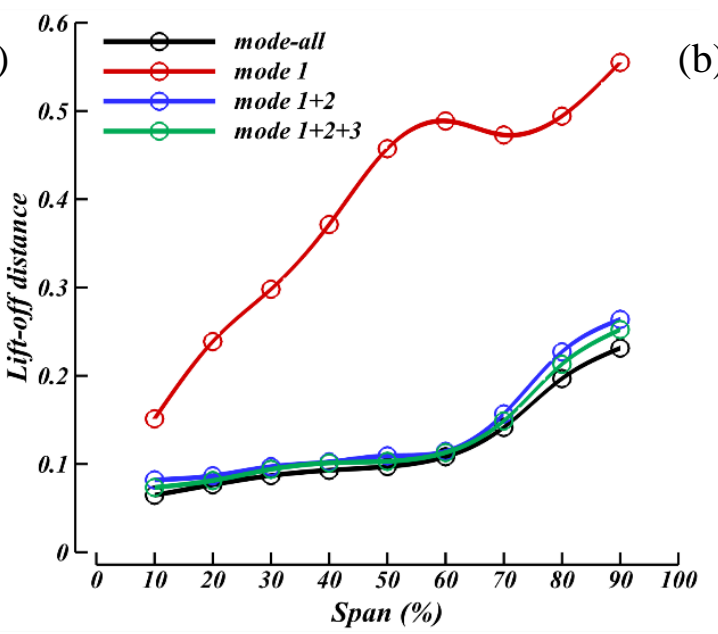

(b)

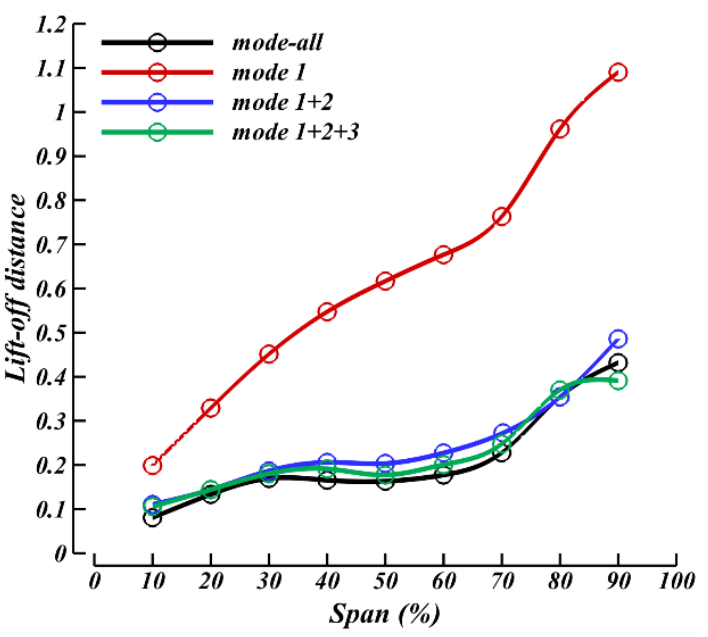

Figure 5-7. Distances of LEV center to the wing surface (lift-off distances) at (a) $t / T=0.25$ and (b) $\mathrm{t} / \mathrm{T}=0.75$ for mode-all, mode 1 , mode $1+2$ and mode $1+2+3$ cases.

For the mode-all, mode $1+2$ and mode $1+2+3$ cases, the lift-off distances are quite similar. The differences are less than $8 \%$ and $10 \%$ for each span location at mid downstroke and mid upstroke, respectively. More importantly, at both time instances, two distinct ranges of the lift-off distances can be observed. The first range is from $10 \%$ to $60 \%$ span, where the lift-off distance increases slowly. It suggests that the LEV attached pretty well with this range. The second range is from $60 \%$ to $90 \%$ span, where a rapid increase in liftoff distance can be observed. The LEV is lifted by the tip vortex and starts to merges with the tip vortex at this point.

The lift-off distances at mid downstroke are almost twice as much as that at mid upstroke for all cases and span locations, which indicates that the LEV attachment at downstroke is much better than upstroke. 

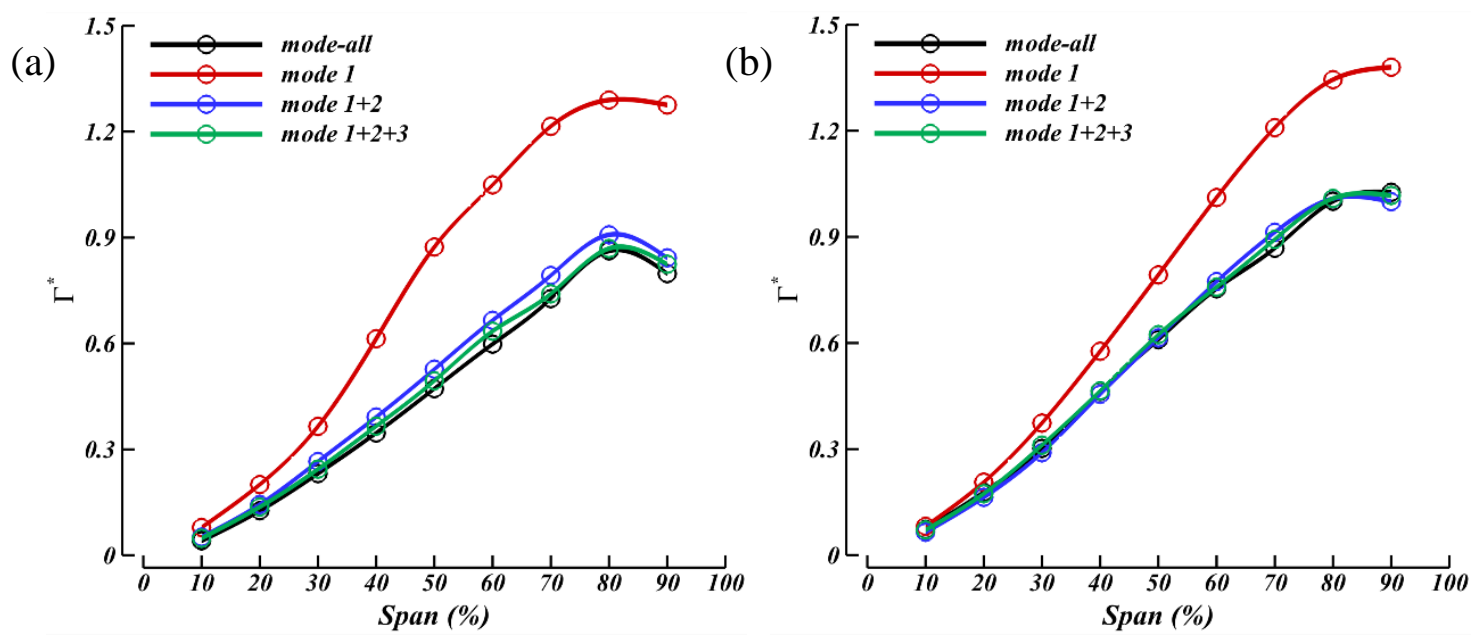

Figure 5-8. LEV circulations at (a) $\mathrm{t} / \mathrm{T}=0.25$ and (b) $\mathrm{t} / \mathrm{T}=0.75$ for mode-all, mode 1 , mode $1+2$ and mode $1+2+3$ cases.

Quantitative measurements of LEV circulation distributions along the wingspan are also performed for all cases discussed above based on the 2D flow slices shown in Figure 5-6. Figure 5-8 shows the corresponding results at mid downstroke $(\mathrm{t} / \mathrm{T}=0.25)$ and mid upstroke $(\mathrm{t} / \mathrm{T}=0.75)$. The circulation is calculated and normalized as follows:

$$
\Gamma^{*}=\frac{c}{U_{r e f}} \iint_{S} \omega \cdot d \mathbf{S}
$$

Where $S$ stands the surface of LEV shapes shown in Figure 5-6; $\omega$ is vorticity on $S$; $U_{r e f}$ is reference velocity, which is chosen as the average velocity of wing mid chord; $c$ denotes the mid chord length. We can see from Figure 5-8 that the LEV circulations of mode-all, mode $1+2$ and mode $1+2+3$ cases are very close. The difference is less than $7 \%$ at mid downstroke and 3\% at mid upstroke. For all cases, the LEV circulations gradually 
increase from the wing root to wing tip and drops a little bit near the wing tip region. Maximum circulation can be observed at around $80 \%$ span, and the corresponding value for mode 1 only case is about 1.5 and 1.3 times larger than that of other three cases at mid downstroke and mid upstroke, respectively.

In summary, we have studied three-dimensional flows around the forewing of a hovering dragonfly in this section, focusing on the effects of model dimensionality. Both qualitative observations and quantitative measurements are performed. The results show that for cases of mode-all, mode $1+2$ and mode $1+2+3$, the wake structures in both far and near field are similar, while significant differences can be found in mode 1 only case. Quantitative measurements of the flow field at two time instances ( $t / T=0.25$ and 0.75$)$ are performed in all cases, including the LEV lift-off distances and circulations. The results show the similarity in all cases except for the mode 1 only case, which has much greater LEV lift-off distances and circulations.

\subsection{Dominant Modes Effect}

In previous sections, we have both qualitatively and quantitatively studied the effects of dimensionality on the aerodynamics of the forewing motion of a hovering dragonfly. The results show that the low-dimensional model (mode $1+2$ ), which consists of two dominant SSVD modes, flapping mode (mode 1) and morphing mode (mode 2), is a good approximation of the original motion (mode all). In this section, the aerodynamic effects of those two modes are investigated based on the mode 1+2 case. Modified lowdimensional models, which show different amplitudes or phase difference of the dominant 
modes, are constructed. The modes amplitude is modified by adding weight coefficients to the singular values associated with the dominant modes. Modified low-dimensional models are constructed as follows:

$$
\mathbf{A}_{M}=\left[\begin{array}{cc}
U_{1, r}^{1} & U_{1, r}^{2} \\
U_{1, \theta}^{1} & U_{1, \theta}^{2} \\
U_{1, \phi}^{1} & U_{1, \phi}^{2} \\
\vdots & \vdots \\
U_{\mathrm{m}, r}^{1} & U_{\mathrm{m}, r}^{2} \\
U_{\mathrm{m}, \theta}^{1} & U_{\mathrm{m}, \theta}^{2} \\
U_{\mathrm{m}, \phi}^{1} & U_{\mathrm{m}, \phi}^{2}
\end{array}\right]_{3 m \times 2} \quad\left[\begin{array}{cc}
W_{1} \lambda_{1} & 0 \\
0 & W_{2} \lambda_{2}
\end{array}\right]_{2 \times 2}\left[\begin{array}{ccc}
V_{1}^{1} & \cdots & V_{n}^{1} \\
V_{1}^{2} & \cdots & V_{n}^{2}
\end{array}\right]_{2 \times n}
$$

where $A_{M}$ is the displacement matrix of the modified low dimensional model; $U, V$ and $\lambda$ are from the SSVD results correspond to mode 1 and mode $2 ; W$ is a weight coefficient that applies to the singular value of the deformation mode $\left(\lambda_{2}\right)$ to control the amplitude of deformation. The effects of dominant modes are investigated through simulating unsteady flows of modified low-dimensional wing gaits, which contain the different weight of the deformation modes.

Similar to previous studies on effects of wing dimensionalities, all cases here are studied at a Reynolds number of 1840 and reduced frequency of 0.25 . Results present here have been obtained by simulating the flows over four wing strokes. In computing mean quantities, we have discarded the first stroke, and all plots of instantaneous quantities correspond to the third cycle in the stroke by which time the flow has reached a wellestablished stationary state. 


\subsubsection{Effect of SSVD Mode 1 and Mode 2 Amplitudes}

In this section, we describe the Mode 1 and Mode 2 amplitudes effect of the forewing in hovering dragonfly on the aerodynamic performances. The effects of dominant modes amplitude on the quantitative characteristics of the wing are investigated, including lift production and lift economy.

Several observations on how the mode amplitudes contributes to the performances of the wing can be made from these results. As we can see in Figure 5-9 and Figure 5-10, all the aerodynamic performance curves show two peaks (one peak in each half-stroke). For the Mode 1 amplitudes effect, we can see from Figure 5-9(a) and (b) that the differences between the five cases are small. Relatively large differences can be observed during the first half of the upstroke for the lift production. For the Mode 2 amplitudes effect on lift production (Figure 5-10a), the 100\%, 125\% and 150\% mode 2 cases are similar. Other cases (50\% and 75\% mode 2) are not as good as these three. In downstroke, the peak values of lift coefficients for $50 \%$ and $75 \%$ mode 2 cases are about 1.13 and 1.07 times smaller than other cases, respectively, while in upstroke, they are about 3.32 and 1.36 times smaller. Also, the timing of the peak delays a little bit when more deformation is involved.

For the Mode 2 effects on the power consumption (Figure 5-10b), the amplitudes of the curves decrease when more weight of Mode 2 is involved. In downstroke, the peak values of power coefficients for 50\% mode 2 case are about 1.64 and 2.27 times greater than the $150 \%$ mode 2 case, respectively. 

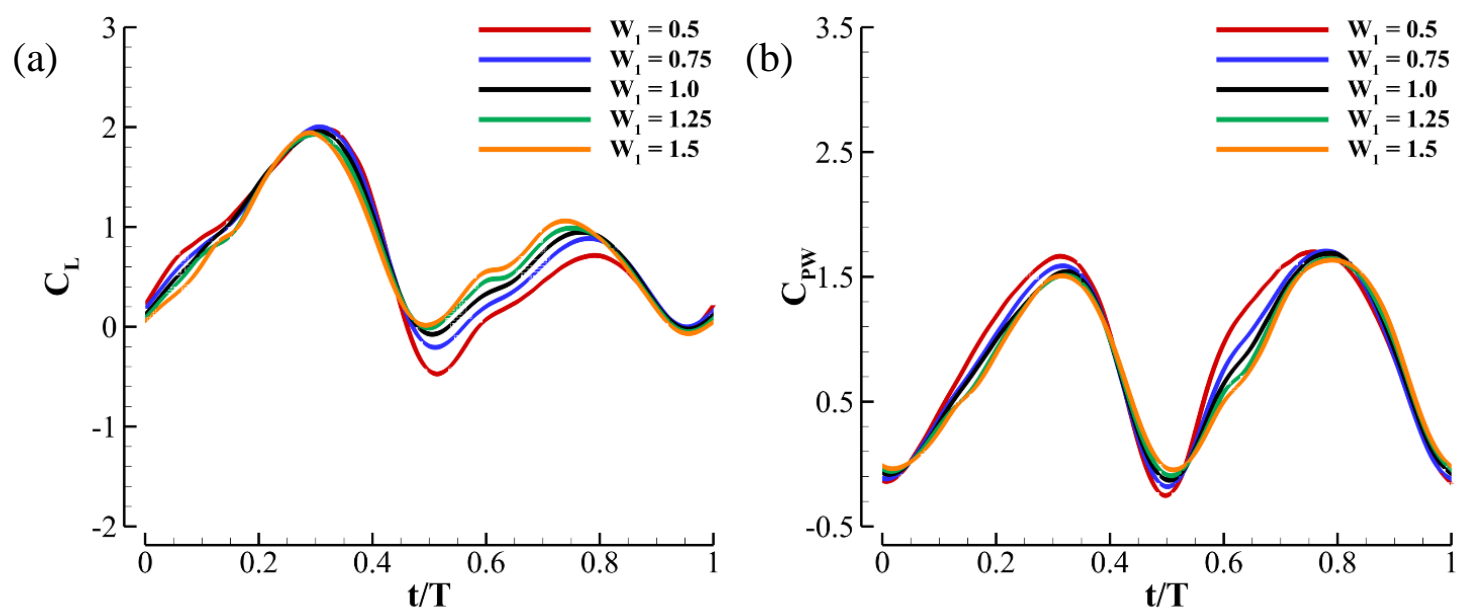

Figure 5-9. Comparison of the time course of aerodynamic performance for the wing gaits with different values of $W_{1}$, while $W_{2}=1.0$. (a) Lift; (b) power.
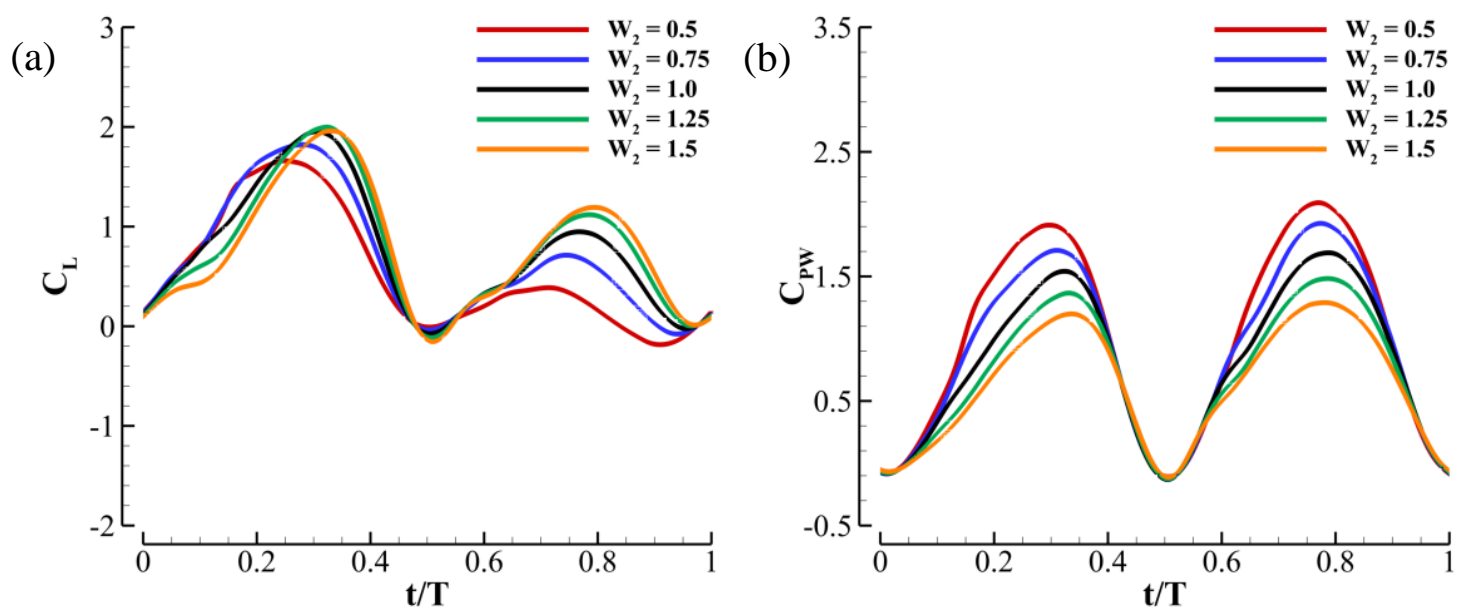

Figure 5-10. Comparison of the time course of aerodynamic performance for the wing gaits with different values of $W_{2}$, while $W_{1}=1.0$. (a) Lift; (b) power. 

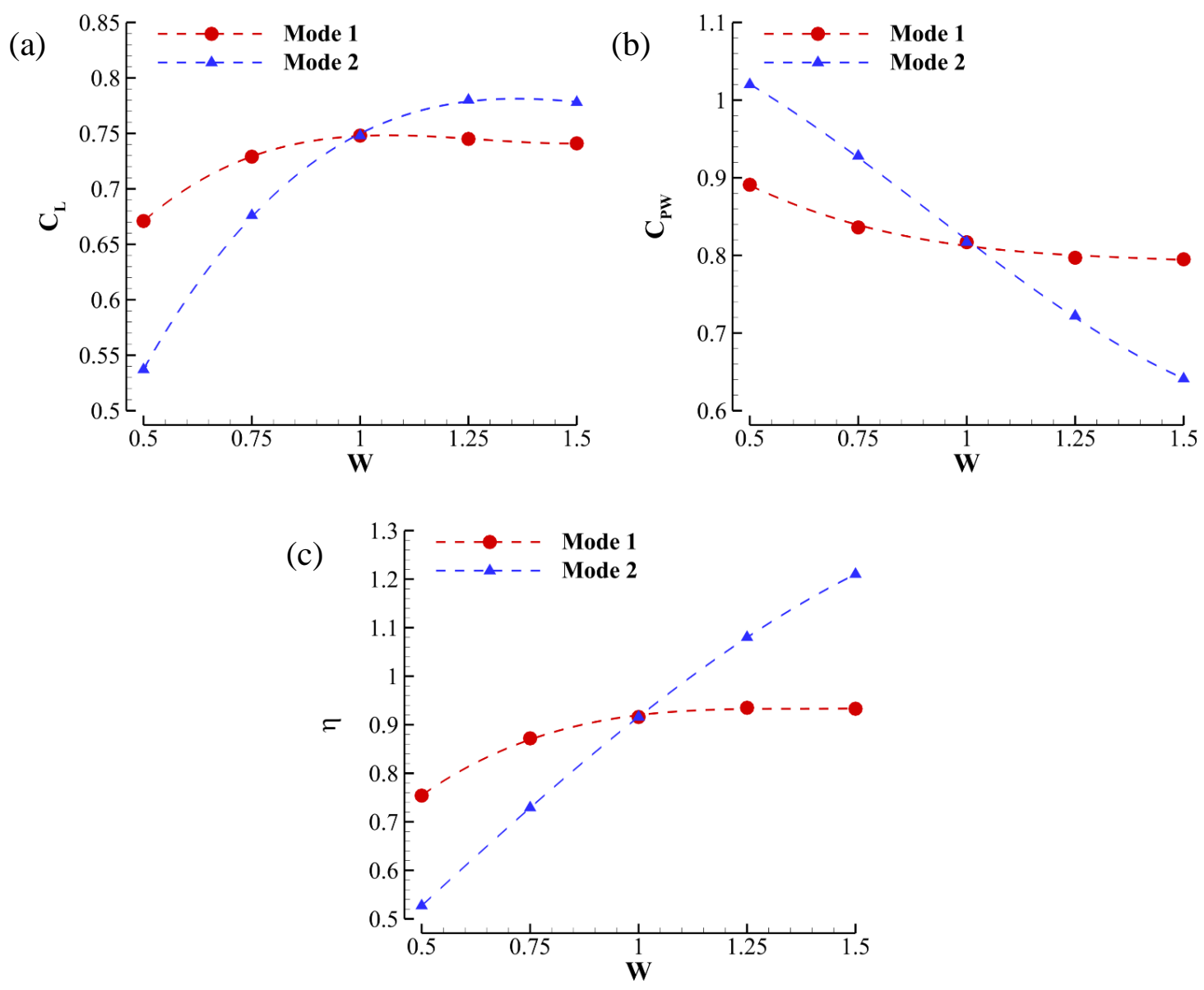

Figure 5-11. Cycle averaged aerodynamic performance of wing gaits with different weight of SSVD Mode 1 and Mode 2. One weight coefficient ranges from 0.5 to 1.5 while another equals to 1.0. (a) Lift; (b) power; (c) lift efficiency.

Cycle averaged aerodynamic performance are shown in Figure 5-11 for all cases discussed above. We can see from the plot that the lift production for the $100 \%, 125 \%$ and $150 \%$ mode 2 cases are almost the same, and the $50 \%$ and $75 \%$ mode 2 cases only have $63 \%$ and $86 \%$ lift production. More interestingly, the cases with more weight of Mode 2 have a better lift economy. The best case, which contains largest weight of Mode 2 (150\% 
mode 2), has the best lift economy. It can produce $98 \%$ of the lift with minimum energy consumption ( $\eta$ is 1.35 times greater than $100 \%$ mode 2 case).

\subsubsection{Effect of Phase Difference between SSVD Mode 1 and Mode 2}

The effect of phase difference between SSVD Mode 1 and Mode 2 is discussed in this section. Figure 5-12 shows the comparison of the time histories of aerodynamic performance for the low dimensional model Mode $1+2$ with different phase difference. We can see that the phase difference shows great influence on the aerodynamic performance, especially for the lift production.
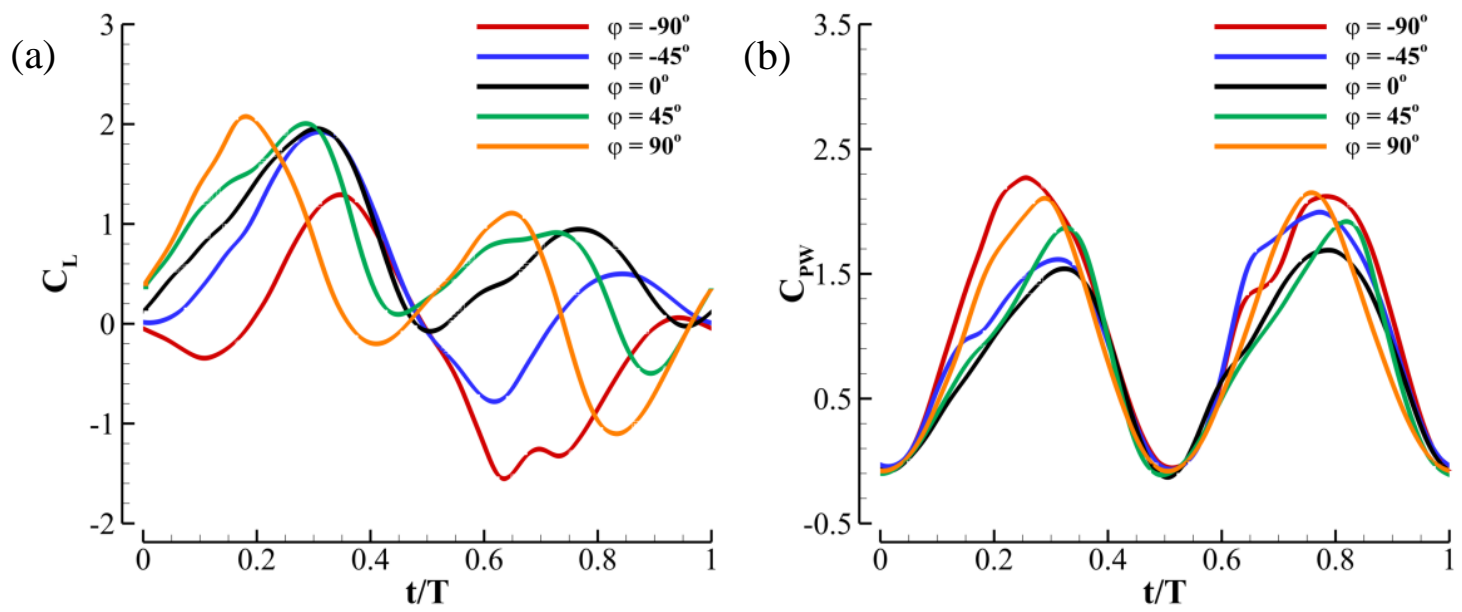

Figure 5-12. Comparison of the time course of aerodynamic performance for the wing gaits with different phase difference between SSVD Mode 1 and 2. (a) Lift; (b) power.

Figure 5-13 shows the cycle averaged aerodynamic performance of those cases. Interestingly, for all the aerodynamic performance, the optimal phase angle is around $20^{\circ}$, 
which suggests that the phase angle of Mode 2 in the low dimensional model Mode $1+2$ is already in an optimal region.
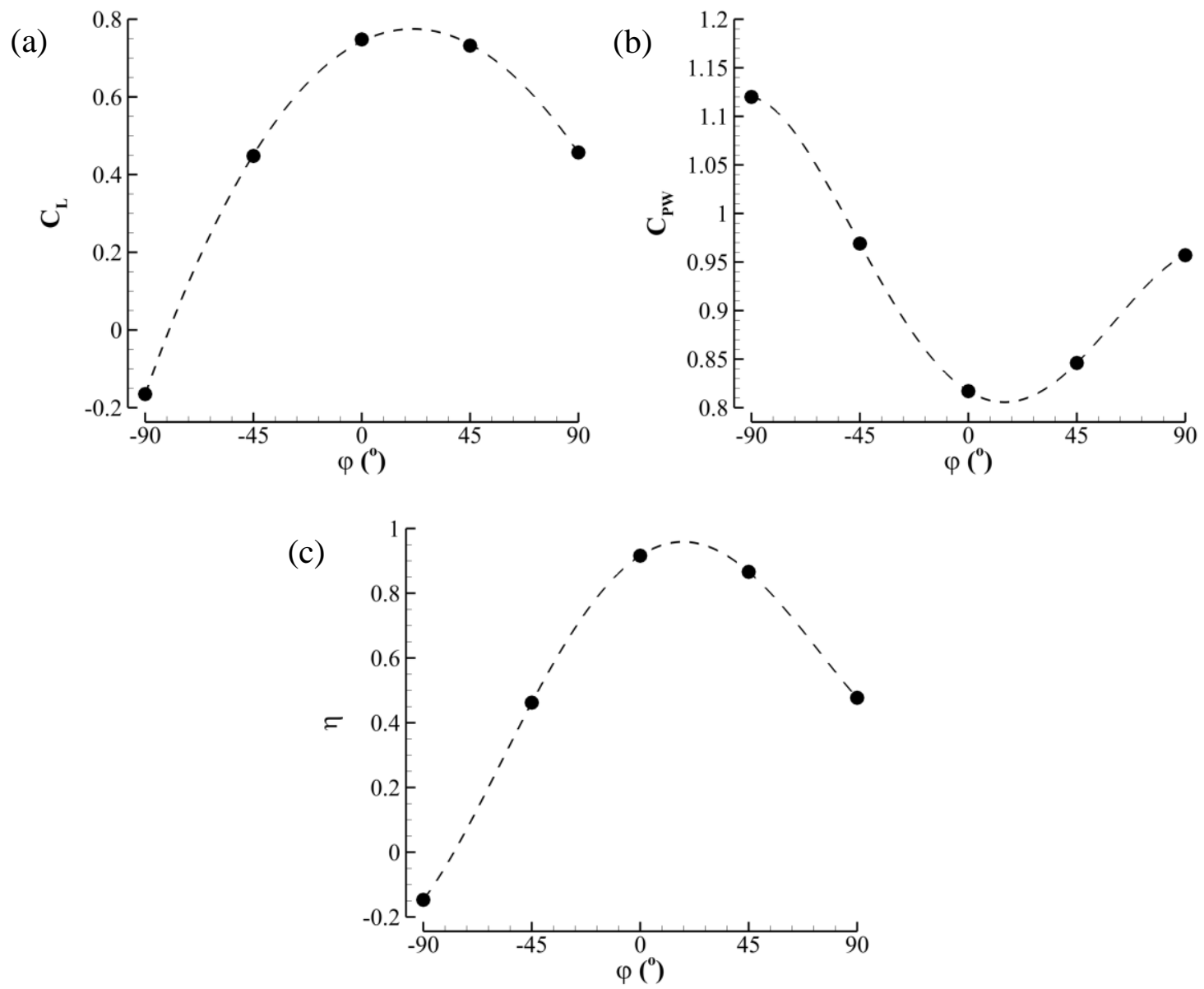

Figure 5-13. Cycle averaged aerodynamic performance of wing gaits with different phase difference between SSVD Mode 1 and 2. (a) Lift; (b) power; (c) lift efficiency.

\subsection{Optimal Low Dimensional Models}

In this section, we investigate the optimal configurations of dominant modes on aerodynamic performance for the dragonfly wing. Figure 5-14 shows the convergence 
histories of the three design variables, $W_{1}, W_{2}$, and $\varphi$. The objective functions are chosen as the cycle averaged lift coefficient and the lift efficiency. The initial guesses of the design variables correspond to the low dimensional model Mode $1+2\left(W_{1}=1, W_{2}=1\right.$, and $\left.\varphi=0^{\circ}\right)$. We can see from the figure that the two optimization case converge within 6 iterations.
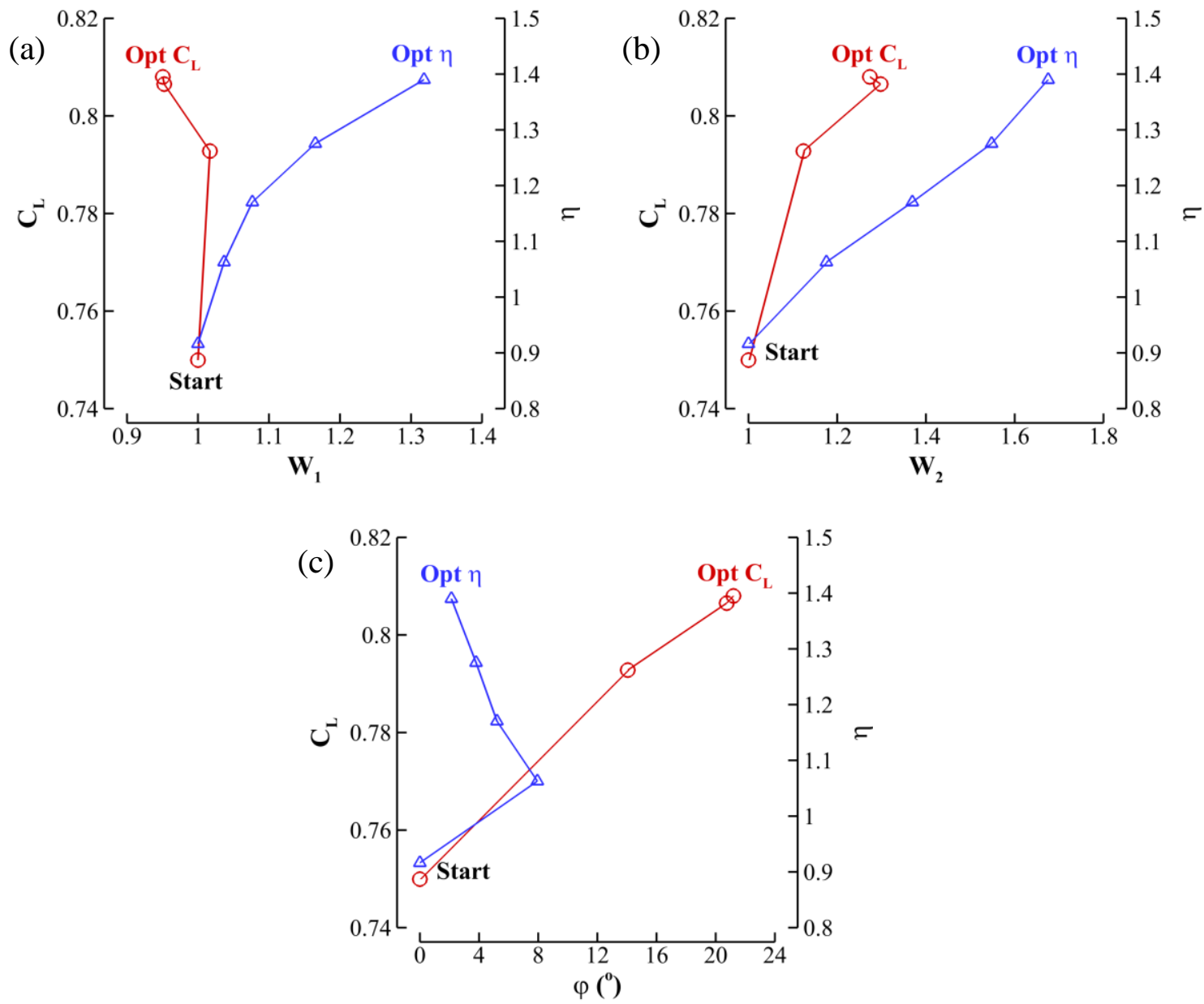

Figure 5-14. Convergence history for design variables (a) $W_{1}$, (b) $W_{2}$, and (c) $\varphi$ of the two optimization cases.

Figure 5-15 shows the time histories of aerodynamic performance of the two optimization cases along with the case of low dimensional model Mode $1+2$ for comparison. 
We can see that the case Opt $C_{L}$ shows largest amplitude of the lift production during the downstroke among the three cases, while the case Opt $\eta$ presents lowest of that. During the upstroke, the case Opt $\eta$ shows largest amplitude of the lift production among the three cases, while the case Mode $1+2$ presents lowest of that. For the power consumption, the case Opt $\eta$ shows the lowest power consumption.
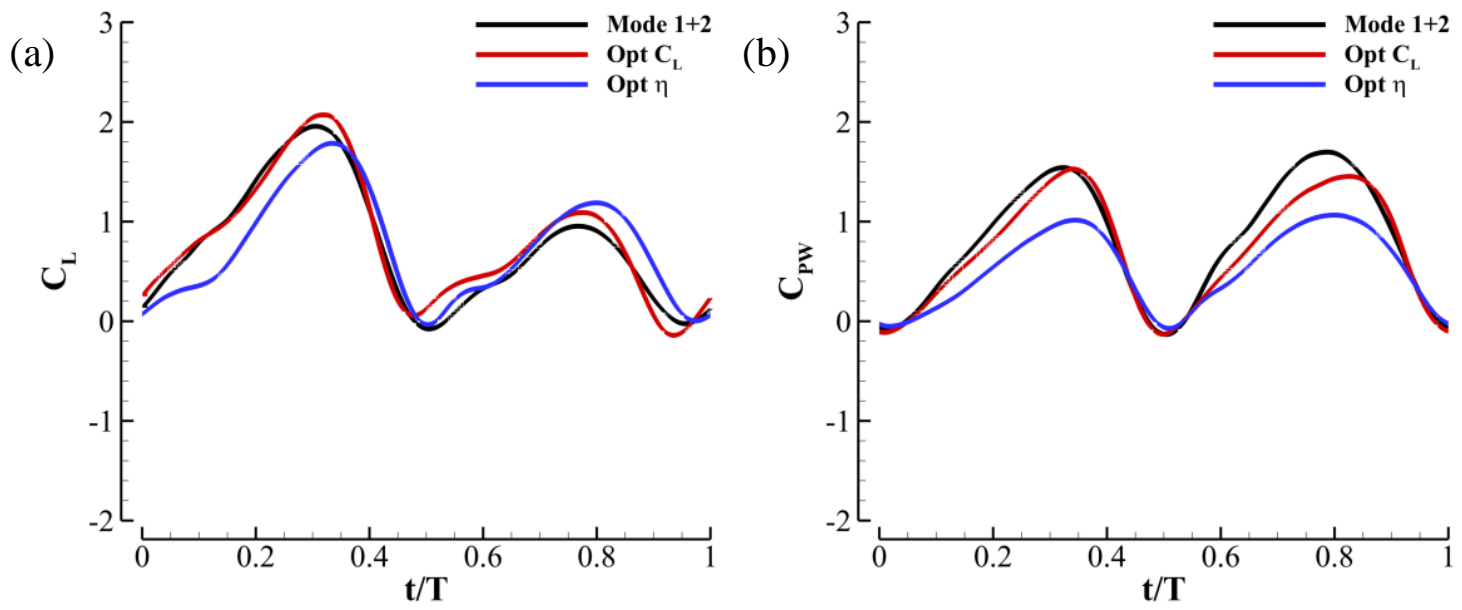

Figure 5-15. Comparison of the time course of aerodynamic performance of Mode $1+2$ and optimized wing gaits. (a) Lift; (b) power.

Table 5-2 lists the cycle averaged aerodynamic performance for the two optimum cases and the case Mode $1+2$. Comparing to the case Mode $1+2$, the thrust production of the Opt $C_{L}$ case is increased by $7.7 \%$, and the propulsive efficiency of the Opt $\eta$ case is increased by $51.6 \%$. The SSVD modes can greatly improve the aerodynamic performance of the flapping wing, especially for the lift efficiency. 
Table 5-2. Cycle averaged hydrodynamic performance of Mode $1+2$ and optimized wing gaits.

\begin{tabular}{lccc}
\hline Cases & $\overline{C_{L}}$ & $\overline{C_{P W}}$ & $\eta$ \\
\hline Mode 1+2 & 0.750 & 0.818 & 0.917 \\
Opt $C_{L}$ & 0.808 & 0.730 & 1.107 \\
Opt $\eta$ & 0.738 & 0.531 & 1.390 \\
\hline
\end{tabular}

Figure 5-16 shows the time averaged lift and power coefficients projected on the wing surface for the three cases. We can see from the figure that most of the lift production and power consumption are produced near the wing tip region for all cases. For the lift production distribution on the wing surface, case Mode $1+2$ show a large concentrating zone, while the case Opt $C_{L}$ show a smaller of that but the values are greater. It suggests that the optimized wing redistribute the surface pressure to let the wing tip generate more aerodynamic force. For the power consumption distribution on the wing surface, the case Mode $1+2$ produces the most power, and the case Opt $\eta$ produces the least. Largest difference of the power consumption locates at the wing tip. It implies that the optimized wing enhances the lift efficiency by reduce the power consumption near the wing tip region.

Figure 5-17 shows the wake structure at the $\mathrm{t} / \mathrm{T}=0.27$ (near the mid downstroke) and $\mathrm{t} / \mathrm{T}=0.73$ (near the mid upstroke) of the three cases. We can see that the general wake structures are similar for all the cases. However, the case Mode $1+2$ shows the largest wing tip vortex and the case Opt $\eta$ shows the least of that. 
(a)

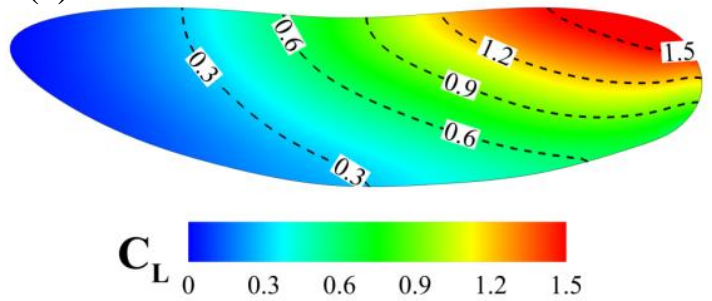

(c)

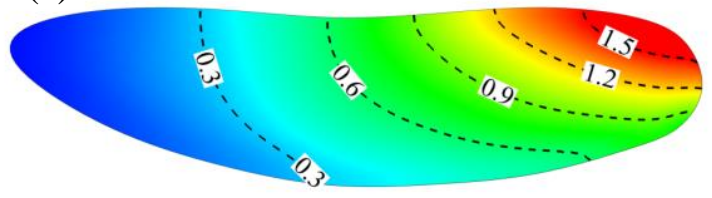

$\mathbf{C}_{\mathbf{L}_{0}}$

(e)

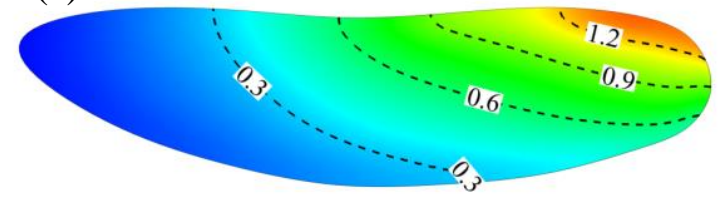

\begin{tabular}{llllll}
$\mathbf{C}_{\mathbf{L}}$ & & & & & \\
\hline & 0.3 & 0.6 & 0.9 & 1.2 & 1.5
\end{tabular} (b)

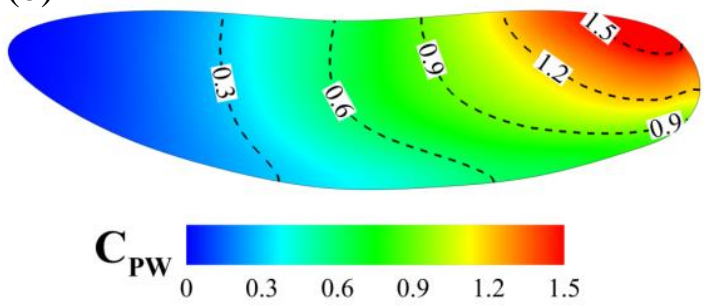

(d)
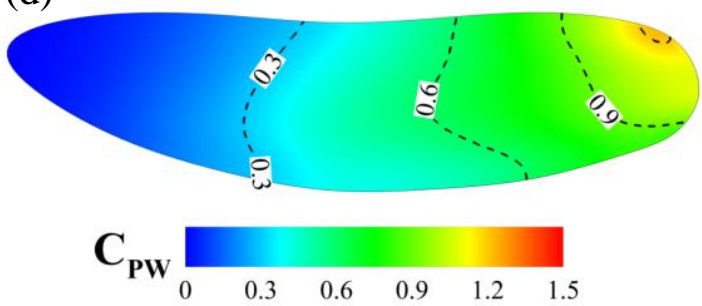

(f)

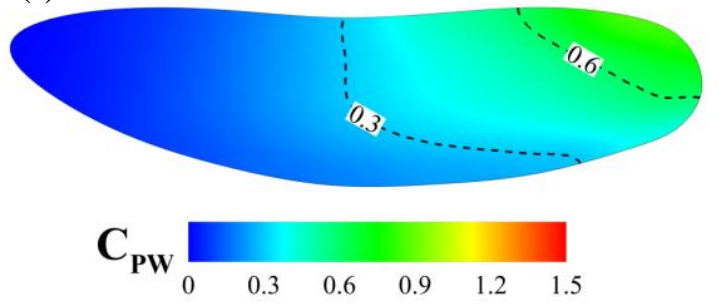

Figure 5-16. Time averaged lift and power coefficients projected on the wing surface for the cases (a, b) Mode $1+2,(\mathrm{c}, \mathrm{d})$ Opt $C_{L}$, and (e, f) Opt $\eta$.

We further cut flow slices along the wing spans for the three cases to study the leadingedge vortex (LEV) structures. Figure 5-18 shows the corresponding results. We can see that the LEV structures are similar for the case Mode $1+2$ and the case Opt $C_{L}$. However, the LEV attachment for the case Opt $C_{L}$ is better near the wing tip region. The case Opt $\eta$ shows smallest shapes of LEV structures among the three cases. 

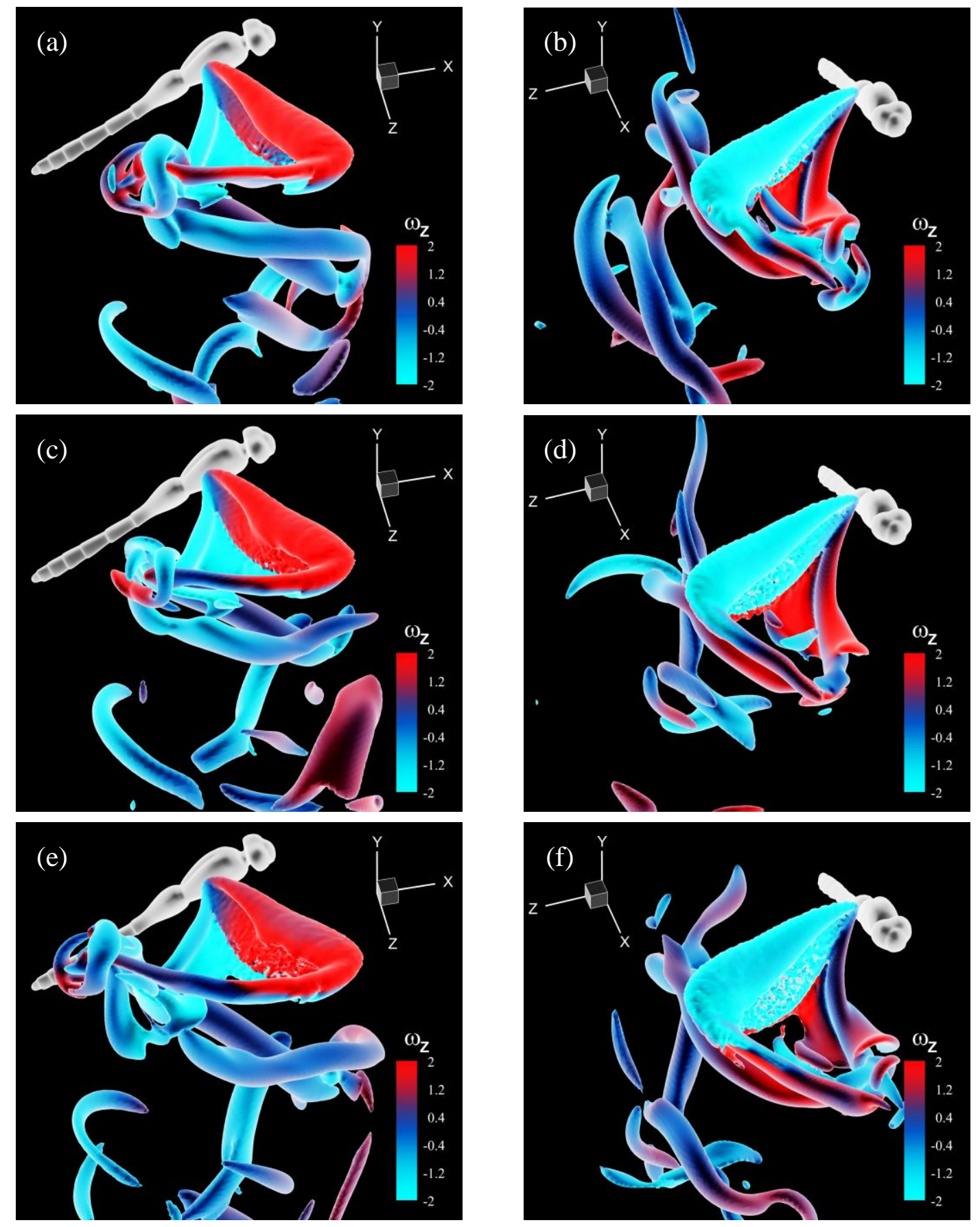

Figure 5-17. Wake structures for the (a) Mode $1+2$, (c) Opt $C_{L}$, (e) Opt $\eta$ at t/T=0.27; wake structures for the (b) Mode $1+2$, (d) Opt $C_{L}$, (f) Opt $\eta$ at t/T=0.73. 

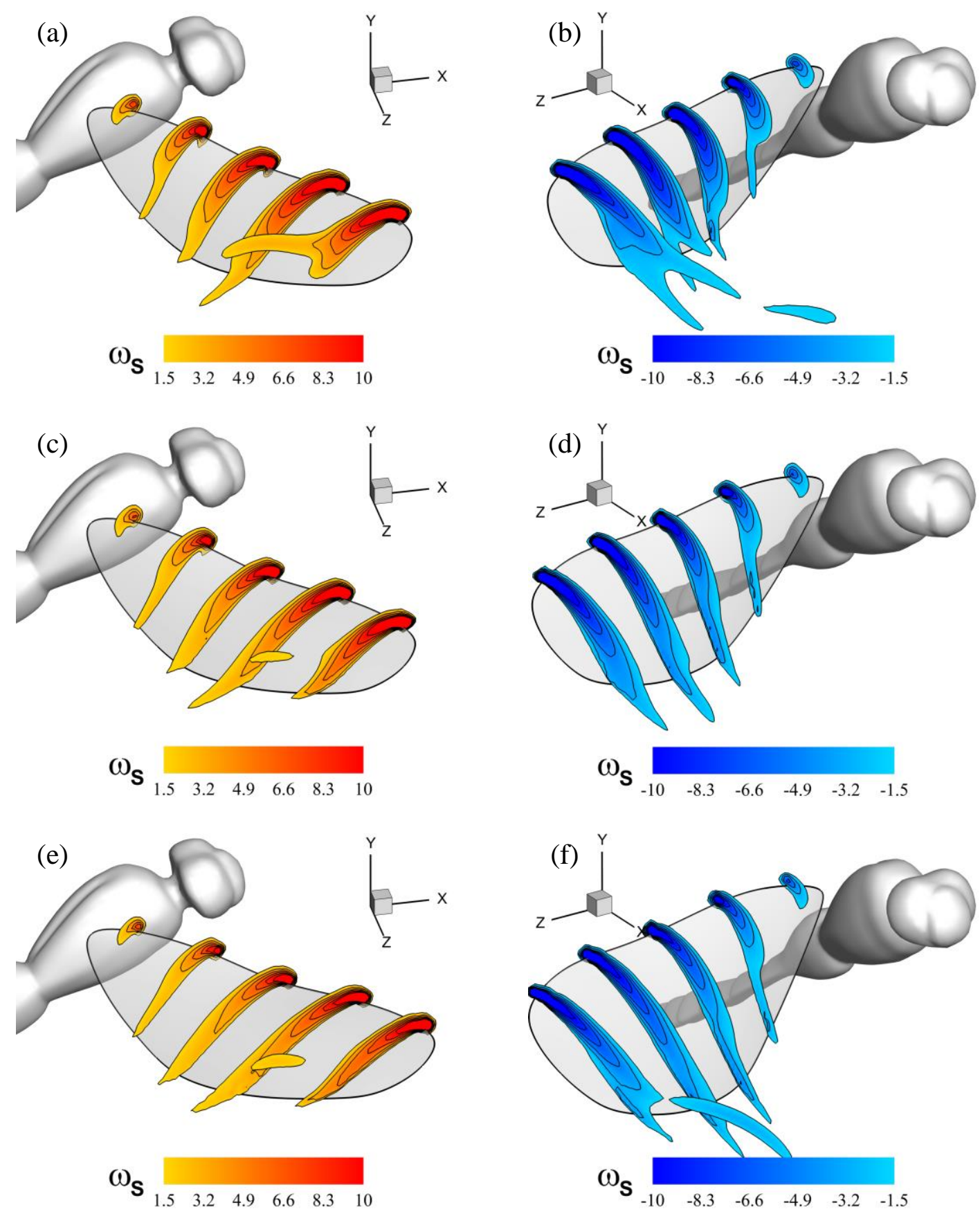

Figure 5-18. LEV structures for the (a) Mode $1+2$, (c) Opt $C_{L}$, (e) Opt $\eta$ at t/T=0.27; LEV structures for the (b) Mode $1+2$, (d) Opt $C_{L}$, (f) Opt $\eta$ at $\mathrm{t} / \mathrm{T}=0.73$. 

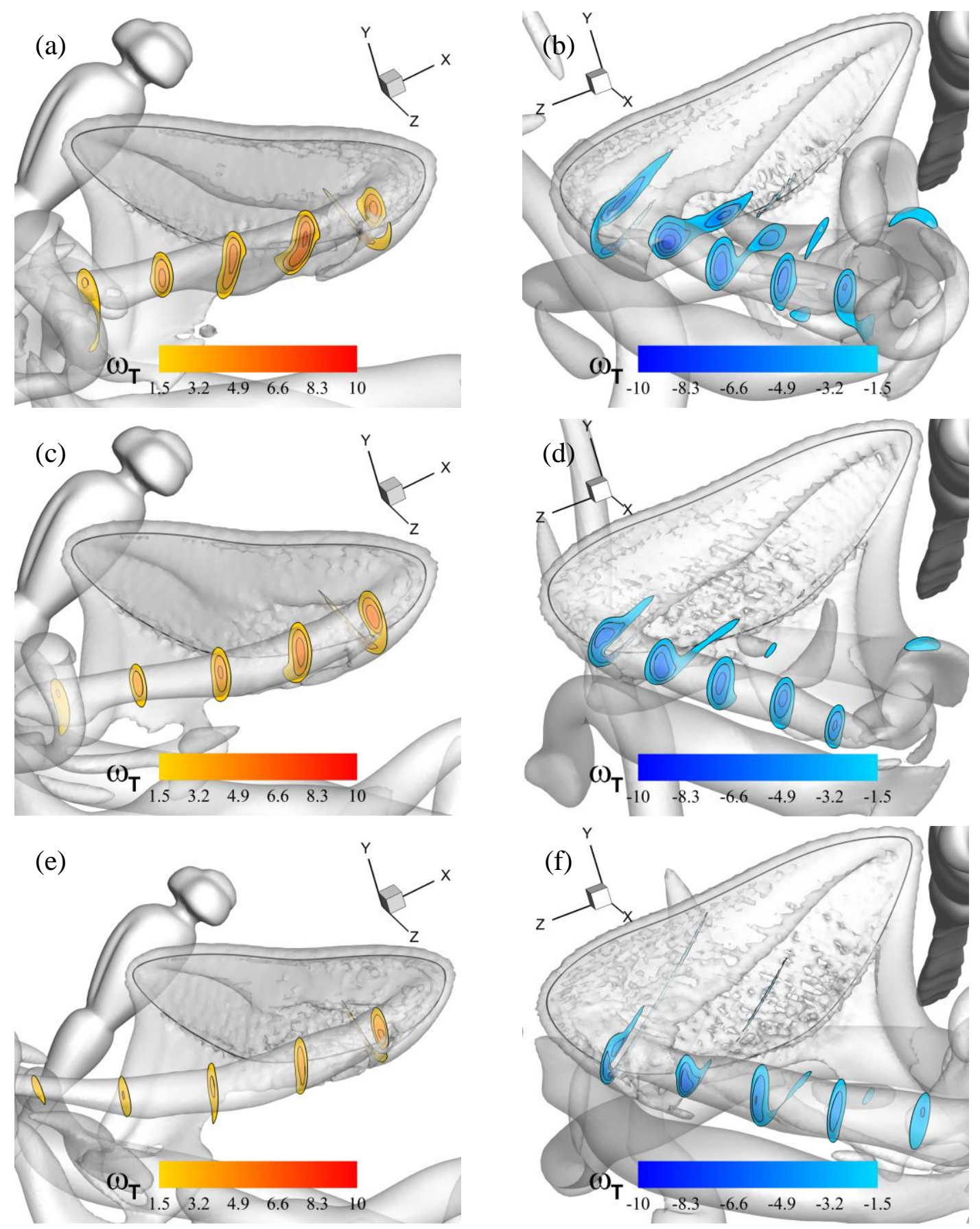

Figure 5-19. TV structures for the (a) Mode $1+2$, (c) Opt $C_{L}$, (e) Opt $\eta$ at t/T=0.27; TV structures for the (b) Mode $1+2$, (d) Opt $C_{L}$, (f) Opt $\eta$ at $\mathrm{t} / \mathrm{T}=0.73$. 
The tip vortex (TV) structures are also studied by cutting flow slices according to the TV tube directions. Figure 5-19 shows the corresponding results. We can see that the case Mode $1+2$ presents largest TV, while the case Opt $\eta$ shows the smallest of that. And the TV structure of the case Mode $1+2$ is much more complicated comparing to the optimal cases, especially at the time instance $\mathrm{t} / \mathrm{T}=0.73$ (near the mid upstroke). In summary, the lift enhancement of the case Opt $C_{L}$ correspond to the improved attachment of LEV near the wing tip region. The lift efficiency improvement of the case Opt $\eta$ related to the reduced wing TV strength.

\subsection{Chapter Summaries}

In this work, the integrated methodology is used to study the complex morphing wing kinematics and the associated aerodynamics of a hovering dragonfly. SSVD analysis of the dragonfly's forewing motion reveals that the complicated wing motion can be represented by a low dimensional model contains two dominant SSVD modes, a flapping mode and a morphing mode. The low dimensional model contains $92 \%$ of the original motion, and can recover up to $96 \%$ of the aerodynamic performance. Parametric studies on the aerodynamic role of the dominant modes reveal that the morphing mode amplitude and phase are critical control parameters to achieve high aerodynamic performance. We further investigate optimal configurations of dominant modes on aerodynamic performance for the dragonfly wing. The corresponding optimized low dimensional wing models, which can beyond biological levels of aerodynamic performance, are obtained. The associated flow mechanisms are found to be the improved LEV attachment and the reduced TV strength. 


\section{The Effect of Chordwise and Spanwise Flexibility on the}

\section{Hydrodynamic Performance of Cetacean Propulsors}

\subsection{Singular Value Decomposition for morphing kinematics analysis of orca fluke}

Singular value decomposition for morphing kinematics analysis (SMOKA) is applied to the fluke motion of a fast swimming orca. In order to focus on the analysis of fluke morphing, we first subtract the mean motion of the fluke, which is the rigid fluke kinematics (denoted as “ $\bar{M}$ "), and then perform the decomposition on the remaining morphing fluke kinematics. 49 snapshots of the fluke motion for one complete flapping cycle are used in the decomposition. As expected, the SVD leads to 49 distinct singular values, and the spectrum for the first ten singular values is shown in Figure 6-1 along with

a cumulative plot for the same data. The normalized singular value for the $\mathrm{k}^{\text {th }}$ mode $\lambda_{k}^{*}$ is defined as:

$$
\lambda_{k}^{*}=\lambda_{k} / \sum_{i=1}^{n} \lambda_{i}
$$

The singular values are normalized by the sum of all singular values. Therefore, the cumulative values sum to unity. A number of interesting observations can be made from this plot. First, the singular value spectrum shows three distinct ranges: the first between mode $1-3$, in which we see a rapid decrease in the amplitude, the second from mode 3 - 5 in which there is a much slower reduction in amplitude and, finally, the range from mode 5 - 49 that has negligible (less than 1\%) total contribution. The rapid initial decrease in the spectrum is significant which suggests that a small number of modes contain most of the essential features of the fluke gait. In fact, the cumulative values show that the first two 
modes capture about $74.3 \%$ of the total motion. In fact, only the first mode captures close to $47.8 \%$ of the motion of the fluke, which is a clear demonstration of the ability of SVD to represent the dataset with the least possible number of modes.

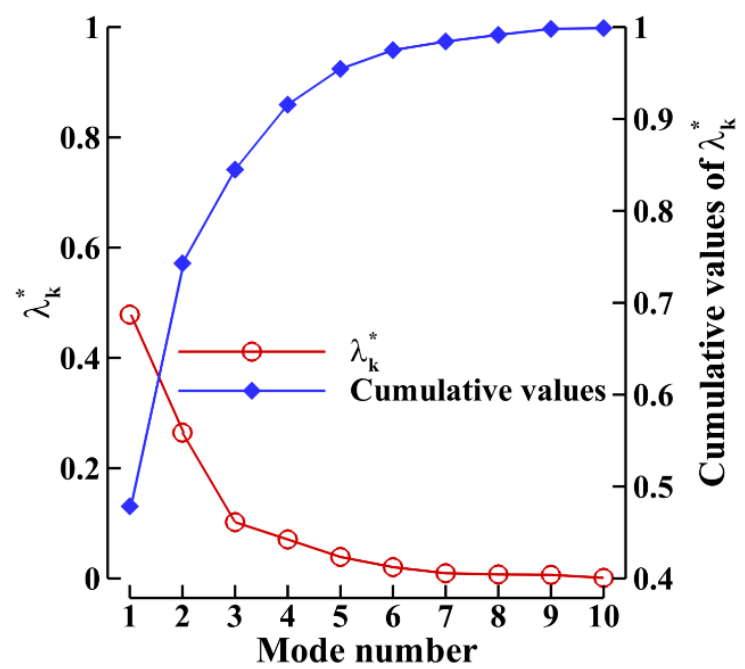

Figure 6-1. The SVD spectrum of the first ten modes for the orca fluke morphing kinematics. The left axis shows the normlized singular values, and the right axis shows the corresponding cumulative values.

The gait corresponding to individual modes can be extracted as described above, and the surface conformations for each of these extracted modes are then constructed using the original fluke mesh with triangular elements. The first two modes are highly distinct and relatively easy to interpret, and we briefly describe the key qualitative features of these modes. Mode 1 involves very large deformation in spanwise direction, which is called the 'spanwise morphing' mode. Mode 2 presents large deformation in chordwise direction, which is called the 'chordwise morphing' mode. Those two modes are primarily a result of flow-induced deformation. The rest of the modes in the spectrum are associated with 
relatively small motions that are not very distinct. We, therefore, do not describe these individually.

\subsection{Effect of Spanwise and Chordwise Morphing on the Hydrodynamic Performance}

In this section, the effect of spanwise and chordwise morphing on the hydrodynamic performance of the orca fluke is discussed. We reconstruct five computational models based on the SVD modes described above. All models contain the rigid fluke kinematics $(\bar{M})$ and different combinations of the SVD modes are added accordingly. The first model is the rigid model (denoted as "Rigid"), which only contains the rigid fluke kinematics. The second model is the spanwise morphing only model (denoted as " $\mathrm{S}$ "), in which the spanwise morphing mode (SVD mode 1) is added. The third model is the chordwise morphing only model (denoted as "C"), in which the chordwise morphing mode (SVD mode 2) is added. The fourth model is the spanwise and chord morphing model (denoted as "SC"), in which both the spanwise and chordwise morphing mode are added. The fifth model is the original model (denoted as "Original"), in which all SVD modes are added. We lower the Reynolds number to 800 (based on the incoming flow velocity and the fluke chord length) in the following discussions due to the computational difficulties. However, the hydrodynamic performance and flow structures are dominated by the unsteady flapping motion of the orca fluke, and it is reasonable for us to perform the analysis in a lower Reynolds number.

Figure 6-2 shows the time course of the thrust and power coefficient for the five computational models. For thrust production, we can see that all five cases show one peak 
in downstroke and two peaks in upstroke. And largest difference happen during the upstroke just after the stroke reversal. For the power consumption, all five cases show one peak in downstroke and one peak in upstroke. And largest different happen near the middle of downstroke.
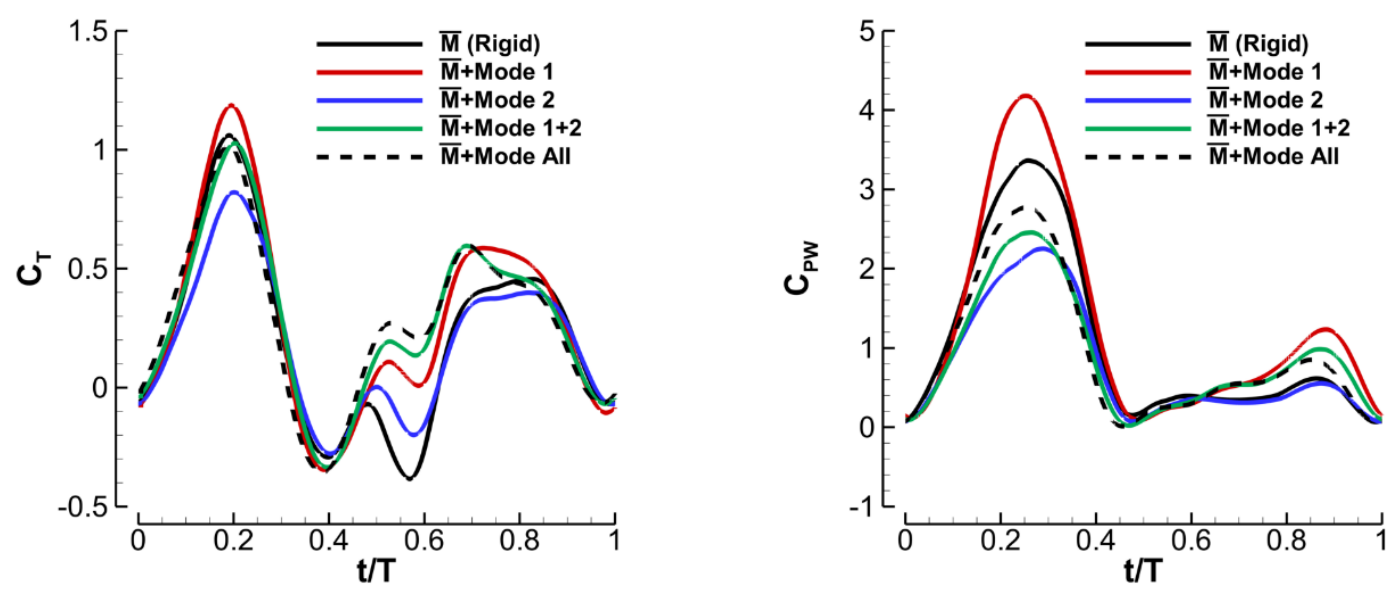

Figure 6-2. Time course of the (a) thrust and (b) power coefficient for the five computational models.

Time averaged values of the hydrodynamic performance are listed in Table 6-1. We can see that the case Rigid shows small thrust production and propulsive efficiency. The thrust production improves about $37.7 \%$ when the spanwise morphing is added, and the propulsive efficiency improves about $24.2 \%$ when the chordwise morphing is added. Consider the case SC, which contains both the spanwise and chordwise morphing, the thrust production is increased about $36.7 \%$ and the propulsive efficiency is increased about $62.7 \%$. The hydrodynamic performance of the case SC is very similar to the case Original, 
which implies that other small modes (mode 3, 4, etc...) do not contribute much on the hydrodynamic performance. The performance improvements are due to the involvement of the fluke surface morphing modes (mode 1 and mode 2).

Table 6-1. Cycle averaged hydrodynamic performance of the five computational models.

\begin{tabular}{lcccc}
\hline \hline Models & $\delta^{*}(\%)$ & $\overline{C_{T}}$ & $\overline{C_{P W}}$ & $\eta(\%)$ \\
\hline 1: Rigid & 0 & 0.215 & 1.067 & 20.15 \\
2: S & 47.8 & 0.296 & 1.310 & 22.60 \\
3: C & 26.5 & 0.195 & 0.779 & 25.03 \\
4: SC & 74.3 & 0.294 & 0.897 & 32.78 \\
5: Original & 100 & 0.289 & 0.929 & 31.11 \\
\hline
\end{tabular}

\subsection{Effect of Spanwise and Chordwise Morphing on the Wake Structure}

In this section, the wake structures of three computational modes, model S, C, and SC, are discussed. Figure 6-3(a) and (b) show the near wake structures at $t / T=0.58$ of case $\mathrm{C}$ and case SC, respectively. At this time instance, the two cases show largest difference in thrust production. We can see from the plots that the fluke surface near the mid span and close to the leading edge shows much greater thrust production for the case SC. This can be explained by the much greater leading edge vortex strength near the mid span for the case SC. We further conclude that the leading edge vortex strength is improved due to the spanwise morphing, which leads to the improvement of the thrust production. 

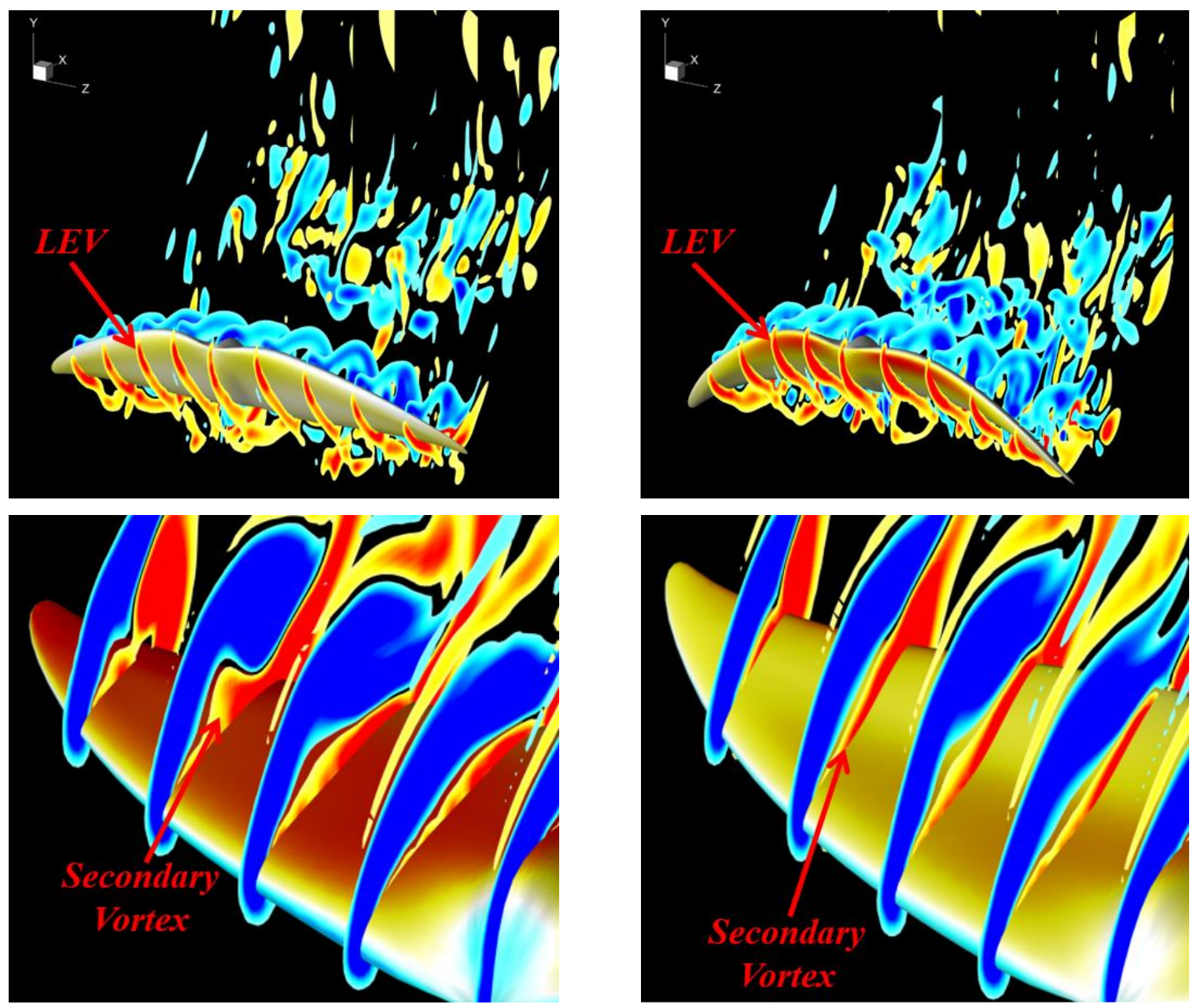

Figure 6-3. Near wake structures of (a) case $\mathrm{C}$ and (b) case $\mathrm{SC}$ at $\mathrm{t} / \mathrm{T}=58$, the fluke surfaces are colored by $C_{T}$; near wake structures of (c) case $\mathrm{S}$ and (d) case SC at t/T=0.25, the fluke surfaces are colored by $C_{P W}$. The wake structures are colored by spanwise vorticity.

Figure 6-3(c) and (d) show the near wake structure at $\mathrm{t} / \mathrm{T}=0.25$ of case $\mathrm{S}$ and case $\mathrm{SC}$, respectively. At this time instance, the two cases show largest difference in power consumption. We can see from the plots that the entire fluke surface shows much greater power consumption for the case $\mathrm{C}$, where several strong secondary vortices can be 
observed just beneath the leading edge vortices. Those secondary vortices can lift up the leading edge vortices to degrade their attachment to the fluke surface, which leads to much greater power consumption. We conclude that the chordwise morphing can prevent the formation of large secondary vortices to improve the attachment of leading edge vortices, thus, improve the propulsive efficiency.
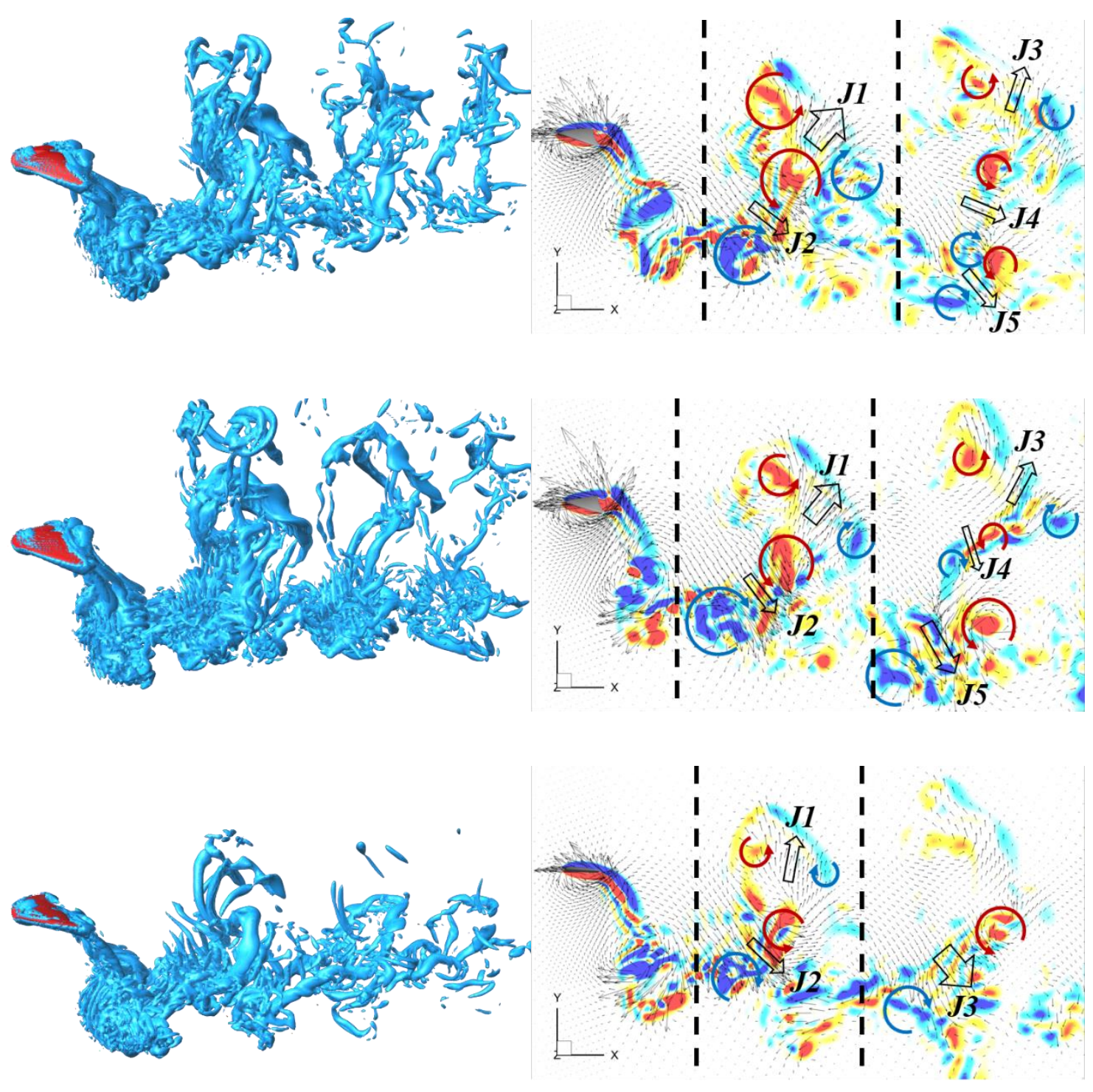

Figure 6-4. Far wake structures at $\mathrm{t} / \mathrm{T}=1.0$ of the (a) case $\mathrm{SC}$, (b) case $\mathrm{S}$, and (c) case $\mathrm{C}$; the corresponding 2D flow slices at mid span of the fluke for (d) case SC, (e) case S, and 
(f) case C. The 2D flow slices are colored by spanwise vorticity and the arrows are vector field of the fluid velocity.

Figure 6-4 shows the far wake structures and the corresponding 2D flow slices of the three cases. We can see from Figure 6-4(a, b, c) that the far wake structures are very complex and some different features among the three cases can be observed. For the case SC, two vortex streets can be observed. For the case S, the lower vortex street is much stronger. And for the case $\mathrm{C}$, the upper vortex street is very weak and cannot be observed in the far wake. For the 2D flow slices of the three cases shown in Figure 6-4(d, e, f), we can see that two flow jets, $\mathrm{J} 1$ and $\mathrm{J} 2$, can be observed in the mid region of the wake. Those two flow jets are induced by two pairs of counter rotating vortices. However, $\mathrm{J} 2$ in case $\mathrm{S}$ is much stronger and pointing more to the vertical direction comparing to that in case SC. It is not good for the propulsive efficiency since the energy is wasted in the vertical direction. Moreover, $\mathrm{J} 1$ in case $\mathrm{C}$ is very weak comparing to that in case $\mathrm{SC}$, which leading to the decrease in thrust production in case $\mathrm{C}$. In the far wake region, the case SC shows three flow jets, $\mathrm{J} 3, \mathrm{~J} 4$, and $\mathrm{J} 5$, which are induced by three pairs of counter rotating vortices. For the case $\mathrm{S}$, the $\mathrm{J} 4$ is much stronger and pointing more to the vertical direction. For the case $\mathrm{C}$, only one flow jet can be observed. The upper two flow jets disappeared due to the weak $\mathrm{J} 1$ in the mid wake region. In summary, we conclude that there are three flow jet exist in the far wake for the case SC and case S. The lower flow jet become stronger and pointing more to the vertical direction due to the absence of the chordwise morphing, which will essentially decrease the propulsive efficiency. The upper flow jet disappears due to the absence of the spanwise morphing, which will essentially decrease the thrust production. 

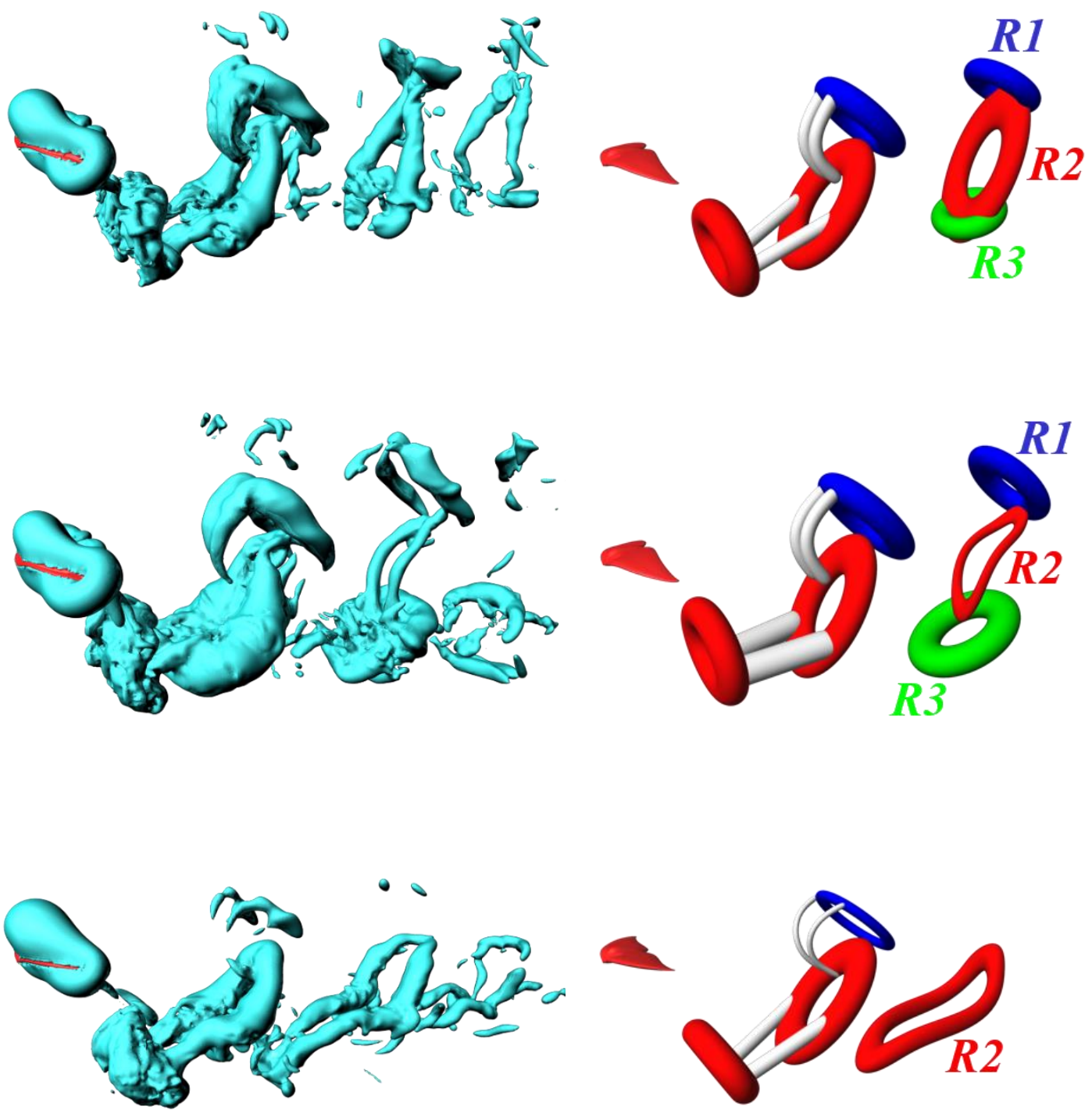

Figure 6-5. Iso-surface plot of the pressure field at $t / T=1.0$ of the (a) case $S C$, (b) case $S$, and (c) case C; the corresponding schematic plots of wake skeleton for (d) case SC, (e) case $\mathrm{S}$, and (f) case $\mathrm{C}$.

In order to qualitative describe the $3 \mathrm{D}$ wake structures of the three cases, the iso-surface of the pressure field are plotted in Figure 6-5(a, b, c). The corresponding schematic plots 
of the wake skeletons are shown in Figure 6-5(d, e, f). We can see in the plots that a unique tri-ring wake structure can be observed in case SC and case S. The R2 is weaker and R3 is much stronger in case $\mathrm{S}$ due to the absence of the chordwise morphing. For the case $\mathrm{C}$, only one vortex ring can be observed in the far wake due to the absence of the spanwise morphing. Those vortex ring structures are closely related to the flow jets shown in Figure 6-4(d, e, f $)$, and are the main reason for the performance difference of the three cases.

\subsection{Chapter Summaries}

The integrated approach is used to study the morphing fluke kinematics and the associated hydrodynamics of a fast swimming orca. The SSVD analysis of the orca fluke motion shows that two dominant modes, a spanwise morphing mode and a chordwise morphing mode can be identified. The low dimensional model consist of these two modes (Mode 1+2) contains $94 \%$ of the original motions, and can recover up to $97 \%$ of the hydrodynamic performance. Parametric studies on the hydrodynamic role of the dominant modes reveal that the spanwise morphing mode is critical to achieve high thrust production while the chordwise morphing mode shows significant influence on propulsive efficiency. The associated flow mechanisms are found to be the improved LEV strength due to the spanwise morphing mode and the reduced secondary vortex strength due to the chordwise morphing mode. Further investigation on the far wake structures of the low dimensional mode (Mode 1+2) shows a unique tri-ring wake structure (upper ring: R1; middle ring: R2; lower ring: R3). The R2 is weakened and R3 is strengthened by removing the chordwise morphing mode, which degrades the propulsive efficiency. The R1 and R3 vanish in the 
far wake by removing the spanwise morphing, which decreases the thrust production. More importantly, this tri-ring vortex structure is closely related to the biology of cetaceans. Flow jet induced by R3 indicates additional vertical force production of cetaceans, which help them to go above the free surface to breathe, or to perform aerial jump. 


\section{Asymmetric Three-dimensional Wake and Aerodynamic Forces of a Maneuvering Hummingbird}

Hummingbirds perform turning maneuvers as often as they hover or cruise, especially when they need to forage from one location to another. However, to date, turning flight has received little attention and most previous studies are primary focused on hovering, forward flight and backward flight, using a variety of techniques. For instance, Warrick and his colleagues measured the wake of hovering rufous hummingbirds using digital particle image velocimetry (DPIV) in two-dimensional (2-D) vertical planes at Reynolds number around $3000[125,126]$. Their DPIV results revealed evidence of leading-edge vortex (LEV) over the wing surface for lift production as widely observed in insect hovering. The bound circulation did not shed as a vortex during the whole flapping cycle. The strength of LEV circulation at different spanwise locations are calculated from 2-D planar flow field images. In general, the mean circulation generated during the downstroke is 2.1 times of that during the upstroke. In another experimental attempt, Altshuler et al. employed particle image velocimetry to attain detailed horizontal flow measurements of hovering hummingbirds (C. anna) at multi-levels [127]. Their flow field images revealed distinct vortex loops underneath the wings, and a three-dimensional wake structure model presenting the primary flow pattern is proposed. In addition to these experimental studies, numerical simulations have been performed for a ruby-throated hummingbird in hovering flight at $\mathrm{Re}=3000$ [128-130]. Similar as observed in [126], the lift production during the down-stroke is about 2.5 times as much as that in up-stroke. Many aspects contribute to this asymmetry vortical force generation, such as angle of attack, drag-based force, wing- 
wake interaction, etc. Their full-body simulations showed that both effects of wing-wing and wing-body interactions are negligible. As an extension work, Song et al. quantified the performance of the same hummingbird through using a quasi-steady model [129]. This low fidelity model is able to predict overall lift production and consistent with their CFD results neglecting fails to capture detailed force oscillations. By exploring wing-pitching mechanism of hovering between, they concluded that pitch reversal of the hummingbird is driven by the wing inertia and there is no power requirement for substantial elastic energy storage at the shoulder joint [130].

Besides above analysis on hovering, Tobalske et al. studied three-dimensional kinematics of forward flight Rufous hummingbirds in a wind tunnel at varies incoming velocities [131]. They observed that both body incline angle and the stroke plan changed respect to the flow velocity, but not for the wingbeat frequency. Furthermore, altering in other kinematics are also significant with velocity, such as chord angle, angle of attack, wingbeat amplitude etc. Other flight behavior such as backward flight also had been studied by [132] for the species-rich hummingbird. Comparing with hovering and forward flight, the backward flight was characterized by the increment on wingbeat frequency, stroke plane, wing position angle, and upstroke duration. Laboratory tests have shown that hummingbird are also capable to achieve sustained yaw turns by altering wingbeat kinematics [133]. Fast change in wingtip speed heading direction is observed within a small turning radius. Unfortunately, aerodynamic performances were not computed or measured in this work.

Despite these previous efforts, there is no detailed forces, moments and threedimensional flow structure data to achieve a quantitative analysis of hummingbird in a 
turning maneuver. To fill this gap, a high-speed photogrammetry system and threedimensional surface reconstruction technology are used to reveal hummingbird wing kinematics and deformations during a free maneuvering flight. The aerodynamic performance is then studied using an in-house immersed boundary method (IBM) based computational fluid dynamics (CFD) solver. To the best of our knowledge, this is the first study on the unsteady aerodynamics of hummingbird in maneuvering flight.

\subsection{Data Acquisition}

The calliope hummingbirds (body mass $3.4 \mathrm{~g}$, Table 7-1) were captured from the wild under permits from the US Fish and Wildlife Service and Oregon Department of Fish and Wildlife. All housing and experimental protocols were approved by the University of Portland Institutional Animal Care and Use Committee. During captivity, birds were housed in $1 \mathrm{~m} \times 1 \mathrm{~m} \times 1 \mathrm{~m}$ flight cages with ad libitum access to food and water in the form of Nektar-Plus (NEKTON@; Günter Enderle, Pforzheim, Baden- Württemberg, Germany) or a $20 \%$ sucrose solution. We measured the morphology of the birds with their wings spread as in mid-downstroke using standard techniques [134]. Linear measurements (mm) were

obtained using digital calipers and metric rulers. Areas $\left(\mathrm{mm}^{2}\right)$ were measured from digital images using a known pixel-to-metric conversion. Average wing chord (mm) was calculated as wing area divided by wingspan. Aspect ratio (dimensionless) was calculated as wingspan divided by wing chord. Disc loading $\left(\mathrm{N} \cdot \mathrm{m}^{-2}\right)$ was computed as body weight divided by disc area $\left(S_{d}\right)$. For this instance, we assumed $S_{d}=\pi(b / 2)^{2}$, where $b$ stands the 
wing span. Wing loading $\left(\mathrm{N} \cdot \mathrm{m}^{-2}\right)$ was computed using body weight $(\mathrm{N})$ divided by combined wing area, including the projected surface area of the body between the wings.

Table 7-1. Morphological data for the calliope hummingbird.

\begin{tabular}{lc}
\hline \hline Variable & Value \\
\hline Body mass $(\mathrm{g})$ & $3.4 \pm 0.1$ \\
Single wing length $(\mathrm{mm})$ & $47 \pm 1$ \\
Wing span, $\mathrm{b}(\mathrm{mm})$ & $109 \pm 2$ \\
Average wing chord, c $(\mathrm{mm})$ & $12 \pm 1$ \\
Aspect ratio & $9 \pm 1$ \\
Single wing area $\left(\mathrm{mm}^{2}\right)$ & $558 \pm 18$ \\
Combined area of both wings and body & $1346 \pm 108$ \\
$\left(\mathrm{~mm}^{2}\right)$ & $25 \pm 3$ \\
Wing loading $\left(\mathrm{N} \cdot \mathrm{m}^{-2}\right)$ & $3.6 \pm 0.2$ \\
Disc loading $\left(\mathrm{N} \cdot \mathrm{m}^{-2}\right)$ & \\
& \\
Values are mean \pm s.d. $(\mathrm{N}=5)$. & \\
\hline \hline
\end{tabular}

We measured wing and body movement using digital video and three-dimensional (3D) reconstruction $[135,136]$. Digital video recordings, $2 \sim 4$ s in duration, were obtained during longer intervals of sustained flight. We used two synchronized Redlake cameras, a PCI2000 and PCI-500 (Redlake MASD LLC, San Diego, CA, USA) sampling at 500 frames $\cdot \mathbf{s}^{-}$ ${ }^{1}$ and with a shutter speed of 1/2500s. Images were stored to computer using PCI-R v.2.18 software. Flights were illuminated using four 650W halogen lights (Lowel Tota-light, Lowel-Light Manufacturing, Inc., Brooklyn, NY, USA) distributed around the outside of the flight chamber. 
(a)

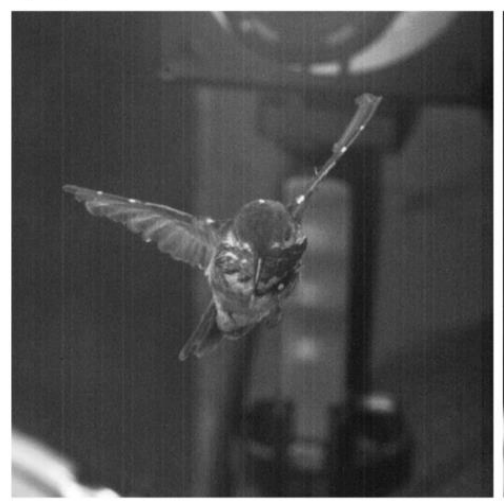

(d)

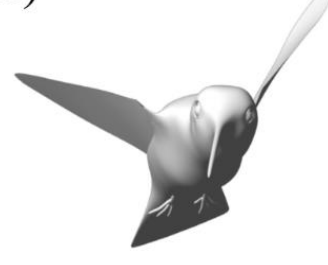

(b)

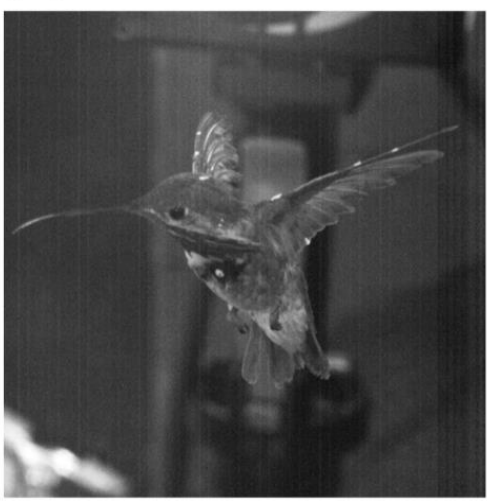

(e)

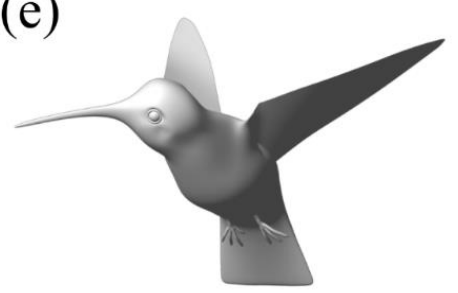

(c)

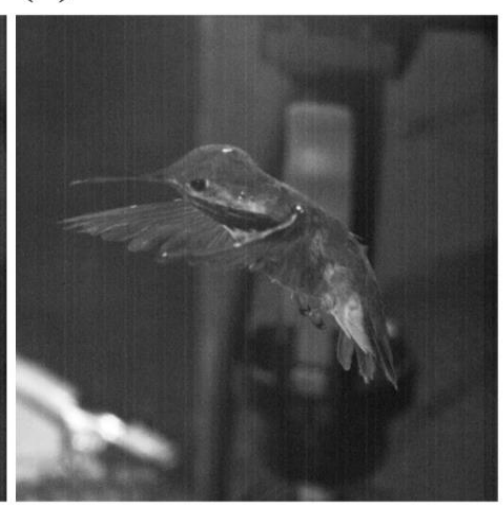

(f)

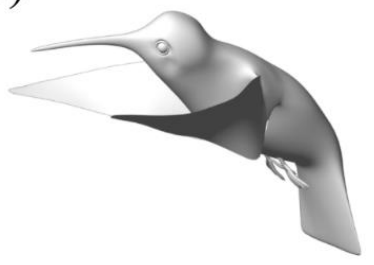

Figure 7-1. Raw pictures of real hummingbird and its reconstructed model at (a, d) $t=0 \mathrm{~ms}$, (b, e) $\mathrm{t}=47 \mathrm{~ms}$, and (c, f) $\mathrm{t}=77 \mathrm{~ms}$.

The hummingbird maneuvering flight is reconstructed using a joint-based hierarchical subdivision surface method. The details about this method and its accuracy can be found in [51]. For completeness, we briefly summarize it here using one wing as an example. Before the videotaping, a fully rigged hummingbird template with virtual joints is built based on anatomical of the hummingbird wing and body. Also, the hummingbird wing was marked with white marker points to facility the three-dimensional surface reconstruction. After the videotaping was done, the pose of the model is adjusted to match one frame of the three directions of high-speed videos by controlling the virtual joints in six degrees of freedom, including three rotations and three translations. Marker points on the wings 
served as references to further tune the location of vertices on the wing template. The wing template was generated with Catmull-Clark subdivision surfaces [137], which is a specific cubic spline surface representation that can generate smooth surfaces from meshes of arbitrary topology [138]. Similar procedure is applied to the hummingbird template frame by frame. As an example, Figure 1(a) shows a raw picture of a hummingbird at the start of downstroke. Figure 1(b) presents a reconstructed model.

\subsection{Computational Setup}

A sharp-interface immersed-boundary method [139] described by [97] and [140] has been used in these simulations. The governing equations are the three-dimensional unsteady, viscous incompressible NS equations:

$$
\frac{\partial u_{i}}{\partial x_{i}}=0 ; \quad \frac{\partial u_{i}}{\partial t}+\frac{\partial u_{i} u_{j}}{\partial x_{j}}=-\frac{1}{\rho} \frac{\partial p}{\partial x_{i}}+v \frac{\partial}{\partial x_{j}}\left(\frac{\partial u_{i}}{\partial x_{j}}\right)
$$

where $u_{i}$ are the velocity components, $p$ is the pressure, $\rho$ and $v$ are the fluid density and viscosity, respectively. The above equations are discretized using a cell-centered, collocated (non-staggered) arrangement of the primitive variables $\left(u_{i}, p\right)$. In addition to the cell-centered velocities $\left(u_{i}\right)$ that satisfy the momentum equations, the face-centered velocities, which satisfy mass conservation, are also computed [95]. A fractional-step method $[141,142]$ is used for the time-advancement of the above equation. The effect of the immersed boundary on the flow is incorporated by using a multi-dimensional ghostcell methodology [139]. The current method has been used to study the aerodynamics of a forward flying cicada [87], and a validation of the method can be found in the same paper. 
More details on the numerical algorithm and immersed-boundary treatment, as well as validations, can also be found in previous publications [97].

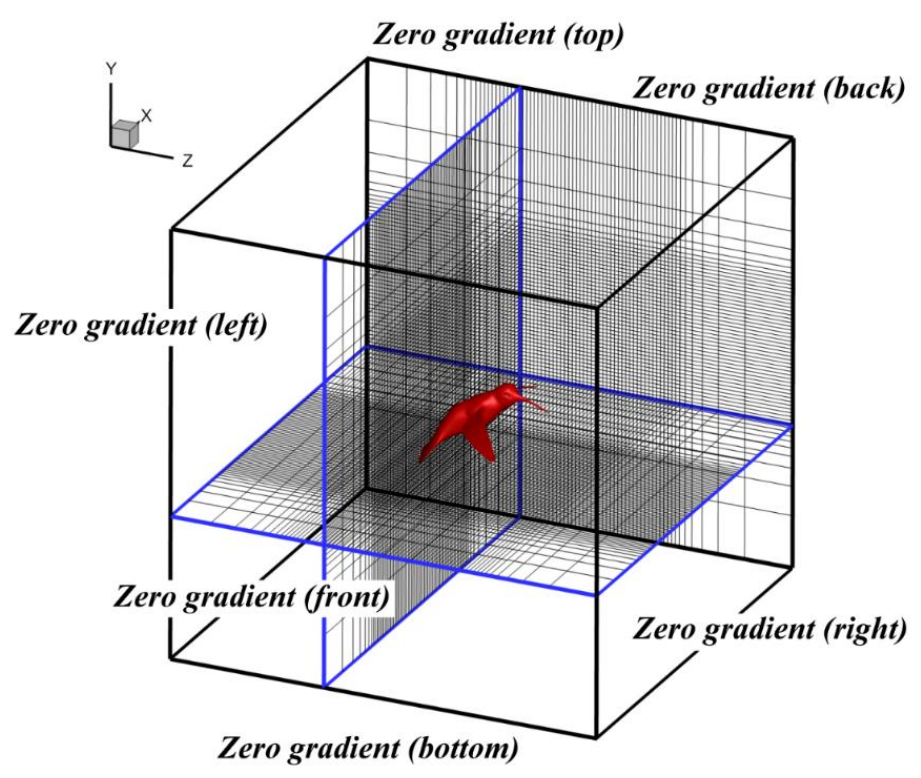

Figure 7-2. Schematic of the computational mesh and boundary conditions employed in the current simulation.

Figure 7-2 shows the constructed realistic full-body model immersed in the threedimensional non-uniform Cartesian grid. $Y$ is the vertical direction with $+Y$ pointing upward. The wings in this study are modelled as deforming membranes and the body is also deformable and both of them are controlled by virtual joints.

The boundary conditions applied in this study is described as follows. On all of the boundaries, gradients of all velocity components are set to zero. The final domain size normalized by the mean chord length $c$ is $20 \times 20 \times 20$ and this large domain ensures that the 
boundaries do not have any significant effect on the computed results. As shown in Figure $7-2$, the grid is designed to provide high resolution in the region around body as well as the wake, which is expected to develop below the body and wings. This grid was developed in an iterative fashion starting from a sequence of coarser grids. Computed results on these earlier meshes were examined and the resolution in selected regions increased until the key features such as forces and vortex structures became essentially independent of the grid. The final grid adopted here has $232 \times 225 \times 232$ points. The grid size in all three directions are around 0.045 chord length, corresponds to 71 grids across the span and 22 across the chord-wise direction on the wing surface.

Three additional simulations on different grids were carried out to assess the convergence of the computed flow. Simulation 1 was carried out on a grid which had $25 \%$ more grid points than the baseline grid and simulation 2 was carried out on a mesh with $25 \%$ less grid points than the baseline grid. Both of these simulations produced a maximum $1 \%$ difference from the baseline in mean lift and root-mean-square (R.M.S.) values of lift and drag. Finally, simulation 3 was carried out on a grid where the number of grid points in each direction around the wing were twice those in simulation 2. This simulation also produces mean lift and R.M.S. lift and drag that are within $1 \%$ of simulation 2 and taken together with the other simulations, provide clear proof of the grid convergence of the current results. 


\subsection{Body and Wing Kinematics}

To better interpret the characteristics of body and wing kinematics, we divided the whole maneuvering process into three phases according to the body yaw angle.

Figure 7-3 shows a schematic plot of hummingbird body yaw motion and the time course of body kinematics. We can see from Figure 7-3(b) that there are in total six strokes of this flight. The hummingbird is first at an "accelerating phase" ( $1^{\text {st }}$ stroke), in which the bird initiates the yaw turn. The body yaw angle start to increase while the other two Euler angles stay unchanged within this phase. After that, the hummingbird enters a "turning phase" for the following three strokes ( $2^{\text {nd }}$ to $4^{\text {th }}$ stroke). The body yaw angle keep increasing and shows an oscillating profile, which indicates an active control of the turning for the hummingbird. The body roll angle shows a little bit decrease and the body pitch angle stays unchanged. The last two strokes of the flight ( $5^{\text {th }}$ to $6^{\text {th }}$ stroke) are called the "recovering phase", in which the body yaw angle stays at a high value and shows a small increase, while the other two Euler angles stay unchanged. The hummingbird is recovering from a turning status to a hovering status within this phase. The path position of this flight (Figure 7-3b) shows a little bit descending motion in vertical direction for the first two strokes, and the motion in horizontal direction is limited.

We have observed similar phases in other maneuvering high-speed videos we shoot. The similarity suggests that the body motion result from similar aerodynamic or dynamic mechanisms. 

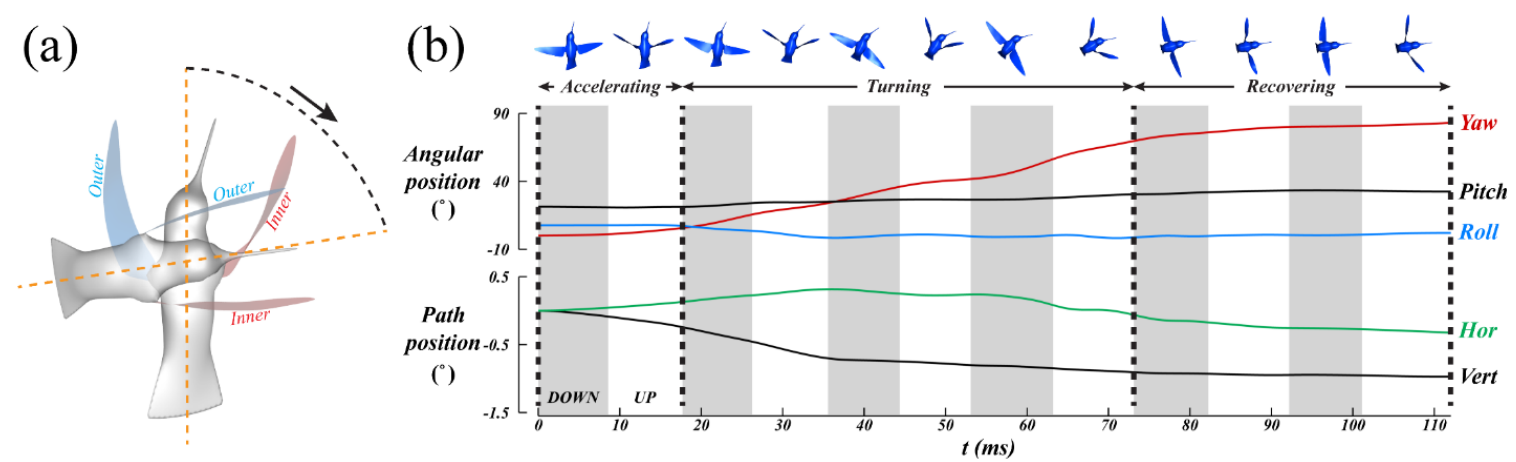

Figure 7-3. (a) Schematic plot showing hummingbird body yaw motion; (b) time course of body of the maneuvering hummingbird. A top view of the hummingbird at the top of the figure indicates the yaw throughout the maneuver. Yaw (red), pitch (black), and roll (blue) histories are shown first, followed by path position histories in horizontal (green) and vertical (black) direction.

In order to determine how hummingbird change wing motion to perform the turning, we also study the wing kinematics of this flight. Figure 7-4(a) shows the three Euler angles defining the wing position in the wing-root coordinate system $\left(X^{\prime} Y^{\prime} Z^{\prime}\right)$, in which the $X^{\prime}$-axis is parallel with the body longitudinal direction, the $Y^{\prime}$-axis is along the lateral direction and the $Z$ '-axis complies with the right-hand rule. The mean stroke plane connected the wing root and wingtips at the start and end of the downstroke. The stroke position angle $\phi(t)$ defines the angular position of the wing in the mean stroke plane, with $0^{\circ}$ aligning with the negative direction of the $Y^{\prime}$-axis. The deviation angle $\theta(t)$ is the angle between the base-towingtip line and the mean stroke plane. The pitch angle $\alpha(t)$ is defined as the angle of the wing chord with respect to the tangent of the wing trajectory. 
(a)

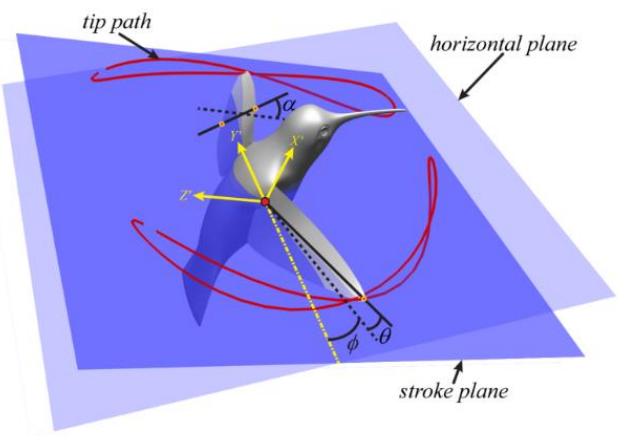

(c)

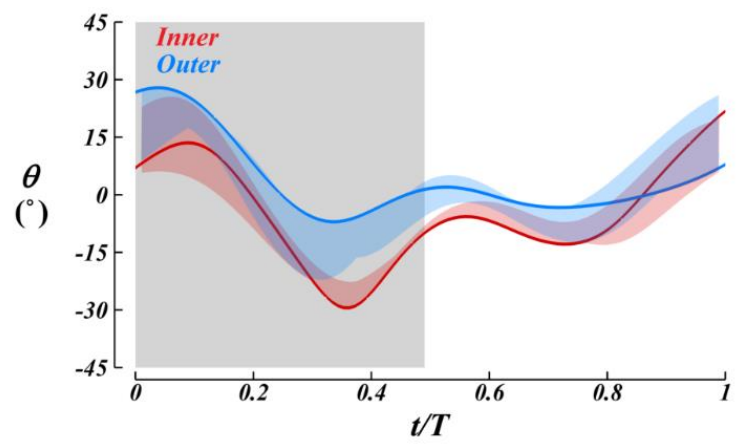

(b)

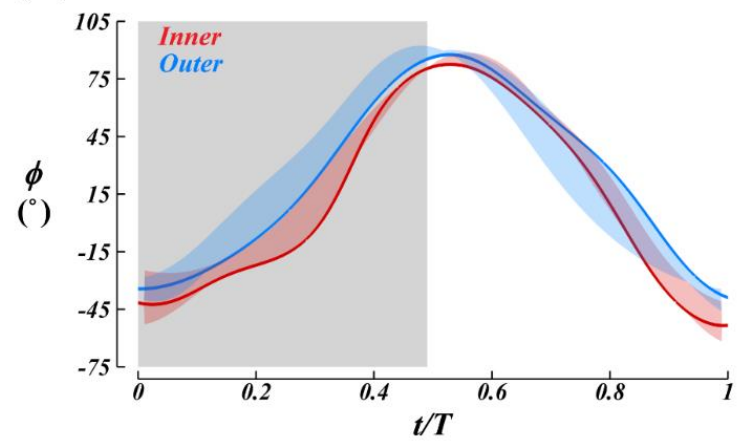

(d)

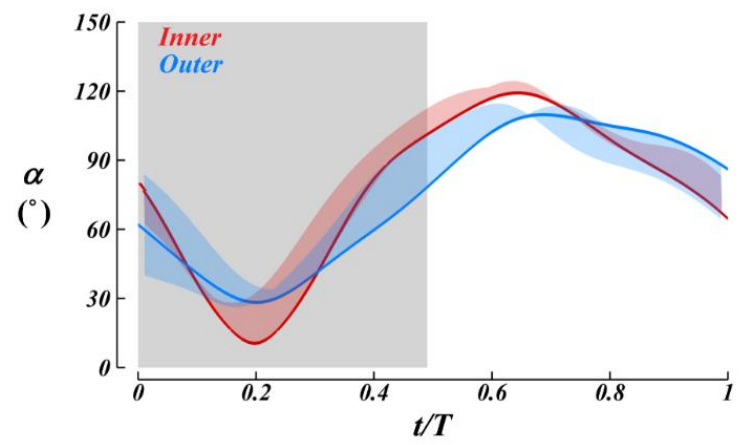

Figure 7-4. (a) Schematic plot showing hummingbird wing Euler angles definition; Time course of wing stroke (b), wing deviation (c), and wing pitch angles (d) during the turning phase. The wing Euler angle histories over the 3rd stroke are shown in solid curves. Angle differences for other strokes in the turning phase with respect to the 3rd stroke are shown as error bars. Red and blue correspond to the inner and outer wings, respectively. Shaded areas stand the downstrokes and unshaded areas stand the upstrokes.

The time course of wing Euler angles during the turning phase are shown in Figure 7-4(b, c, d) according to the above definitions. We can see from the figure that asymmetries of the wing kinematics between inner and outer wings can be identified in the turning phase, especially for the time course of wing deviation angle and pitch angle. As shown in Figure 
7-4(b), the wing stroke angle history shows one peak for a flapping cycle, which locates at the end of the downstroke. The amplitude of the wing stroke angle is around $120^{\circ}$ for both inner and outer wings. However, the inner wing stroke angle is a little bit smaller (7\% smaller) during the downstroke in turning phase, while it is almost the same comparing to that during the upstroke. For the wing deviation angle history, the plot shows two valleys for a flapping cycle, which is located near the mid of downstroke and the mid of upstroke, respectively. Much greater differences can be observed for the wing deviation angle history. The inner wing deviation angle history shows larger amplitude and smaller value in both downstroke and upstroke. This is because of the outer wing stroke plane of the hummingbird is tilted up in the turning phase, and also, the figure eight motion of the outer wing is smaller comparing to that of the inner wing. For the wing pitching angle histories, all plots show one valley and one peak near the mid downstroke and mid upstroke, respectively. More importantly, the wing pitching angle histories show great asymmetry between the inner and outer wings in the turning phase. The valley value of the inner wing pitching angle is much smaller than that of the outer wing, while the peak value is much greater. It leads to the average angles of attack during both the downstroke and the upstroke being much smaller for the inner wing. As shown in Table 7-2, the average inner wing angle of attack is $40 \%$ more than the outer wing angle of attack during the downstroke for the turning phase, while this value is $15 \%$ when considering that during the upstroke. Conventional air foil theory tells us that larger angle of attack would results in larger drag force. We can conclude from the results that the larger inner wing angle of attack during both downstroke and upstroke creates asymmetry in drag production, which is primarily the source of the turning torque generation. Similar drag-based turning torque generation 
mechanism is reported by previous researchers for small insect maneuvering flight [19]. However, in this case, the motion is a pure yaw turn, and also, the mechanism is half-cyclebased.

Table 7-2. Half cycle averaged wing angle of attack for the inner and outer wings of the maneuvering hummingbird.

\begin{tabular}{|c|c|c|c|c|}
\hline \multirow{2}{*}{} & \multicolumn{4}{|c|}{ Average wing angle of attack (degs) } \\
\cline { 2 - 5 } & \multicolumn{2}{|c|}{ Turning $\left(2^{\text {nd }} \sim 4^{\text {th }}\right.$ stroke $)$} & \multicolumn{2}{c|}{ Recovering $\left(5^{\text {th }} \sim 6^{\text {th }}\right.$ stroke $)$} \\
\hline & Inner & Outer & Inner & Outer \\
\hline Downstroke & $73.1 \pm 2.3$ & $52.4 \pm 1.8$ & 53.2 & 53.5 \\
\hline Upstroke & $82.2 \pm 2.1$ & $71.6 \pm 2.5$ & 70.5 & 71.1 \\
\hline
\end{tabular}

Comparing the averaged wing angle of attack for the turning and recovering phase (Table 7-2), we can see that the hummingbird creates the asymmetry by increase the inner wing angle of attack. It suggests that the hummingbird actively control the inner wing kinematics to complete the maneuvering motion in this case.

\subsection{Aerodynamic Force}

CFD simulation is conducted using the kinematics of the wings and the body from the three-dimensional surface reconstruction. The time course of the averaged aerodynamic forces produced in the turning phase (average over the 2nd 4th stroke) are shown in Figure 7-5(a, b). We can see from Figure 7-5(a) that the lift coefficients for both inner wing and 
outer wing show two peaks in one complete stroke. The peaks locate at around $50 \%$ of each half stroke, and it is worth noting that the average values of the lift coefficient in downstroke are much higher than that in upstroke. This conforms to the previous conclusion made on a hovering hummingbird by Song et al. [85]. However, the double peaks in upstrokes, which are also reported by this paper, are not observed in this maneuvering case. A possible explanation for that is, due to the body motion of the maneuvering hummingbird, the effects of wake capture during the stroke reversal is weakened.

(a)

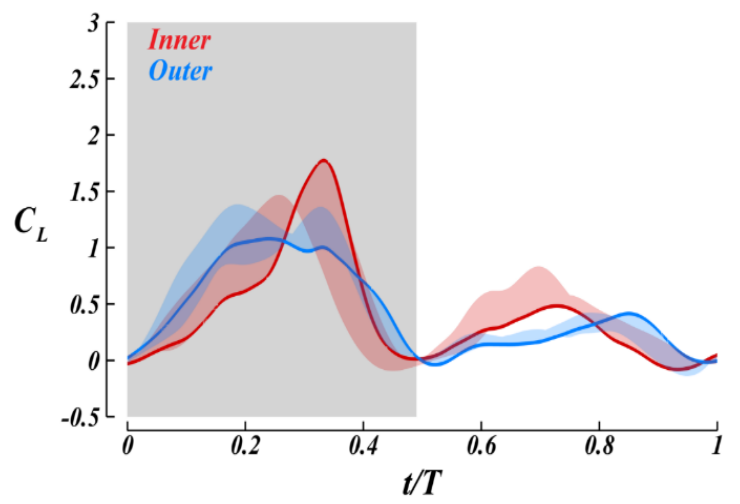

(b)

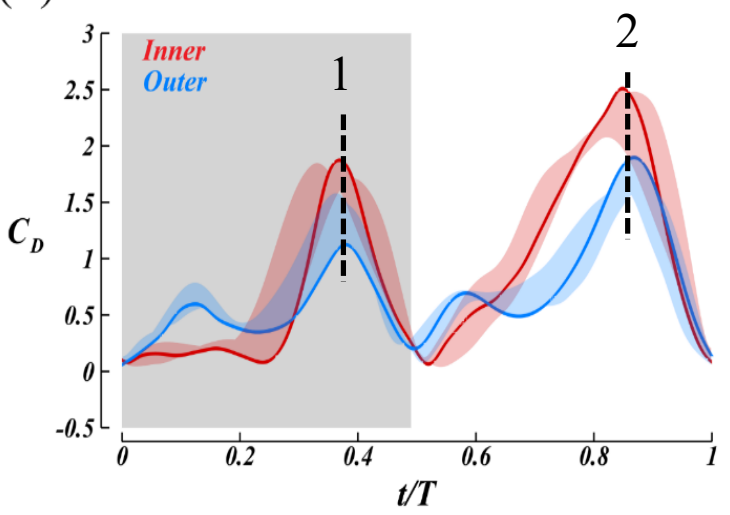

Figure 7-5. Time course of lift (a) and drag (b) coefficients during the turning phase of hummingbird pure yaw turn. The force coefficient histories over the 3rd stroke are shown in solid curves. Force coefficient differences for other strokes in the turning phase with respect to the 3rd stroke are shown as error bars. Red and blue correspond to the inner and outer wings, respectively. Shaded areas stand the downstrokes and unshaded areas stand the upstrokes.

Besides the lift force, horizontal force is also important in maneuvering flight since it can generate torque to drive the turn, especially for the pure yaw turn case. Figure 7-5(b) 
shows the drag coefficient history of this maneuvering flight. We can see from the plot that the horizontal force generated by the inner wing is always greater than that generated by the outer wing. Such force asymmetry in horizontal direction can accelerate the turn in downstroke and damps the turn in upstrokes. This half-cycle-based turning strategy is more flexible comparing to some other nature flyers, like the fruit flies, their wings always generate resultant torque towards the turning direction when they perform the maneuvering flight. It is much easier for the flyer to stop the turn and adopt an alternative flight motion based on what it needs, which is often the purpose of pure yaw turn.

\subsection{Surface Pressure Distribution}

The aerodynamic pressure, which is perpendicular to the local wing elements, is projected in lift and drag directions to indicate the lift and drag force distributions over the wing surface (Figure 7-6). The aerodynamic pressure is non-dimensionalized by the wing load (the ratio of the cicada weight to the total wing surface area). Figure 7-6(a) and (b) show the lift force projection averaged over all downstrokes and upstrokes in the turning phase, respectively. It is interesting to note that the maximum lift force is near the wing tip region (above $90 \%$ wing span) for the inner wing, and is a little bit closer to the mid wing region $(60 \%$ $90 \%)$ for the outer wing. Although the lift force concentrating zone is a little bit different between the inner and outer wings, the overall lift force generated by the two wings are very close (Figure 7-6a). 

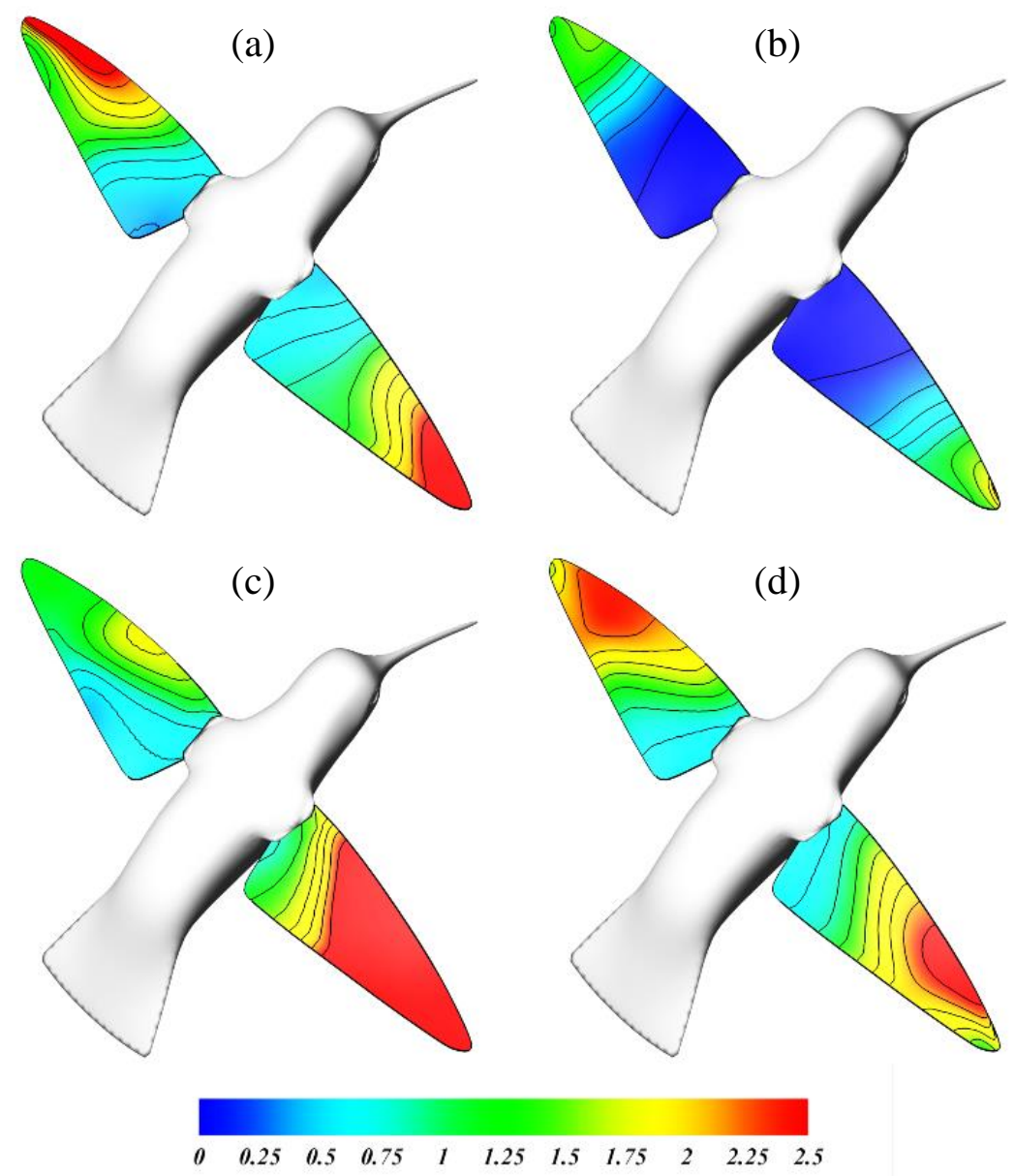

Figure 7-6. Time-averaged surface pressure distribution projected on the lift direction (a, b) and drag direction (c, d); (a) and (c) correspond to the average over all downstrokes in turning phase and (b) and (d) correspond to the average over all upstrokes in turning phase.

\subsection{Vortex Development}

The vortex formation over a stroke cycle (the $3^{\text {rd }}$ stroke) is shown in Figure 7-7, in which the vortex structures are identified by the Isosurface of $\mathrm{Q}$-criterion $(\mathrm{Q}=10)$. The vortex structures are colored by the non-dimensional pressure. The stroke cycle starts from the downstroke. Figure 7-7(a) shows the vortex structures at $t / T=0.24$, which corresponds 
to the peak of the vertical force during downstroke (Figure 7-7a). The most significant vortex structures at this moment is the formation of leading-edge-vortex (LEV). The LEV attaches well to the wing surface. More importantly, the LEV, the tip vortex (TV), the trailing-edge vortex (TEV), and the root vortex (RV) are connected end to end, forming a vortex loop, within which the air moves downward. A previously shed vortex loop (PVL) can also be observed. It was generated during the upstroke prior to this moment, and the PVL is connected to the newly formed vortex loop by the TEV. Similar vortex structures are found in hovering hummingbirds in previous studies [85, 125, 143].

As time advances to $\mathrm{t} / \mathrm{T}=0.33$, as shown in Figure 7-7(b), which corresponds to the peak of horizontal force in downstroke (Figure 7-7b), the wings are near the end of downstroke and rotate rapidly along their own axis. The outer wing LEV is divided into two branches, known as dual LEV [138], and two shed LEVs, SLEV1 and SLEV2, can be identified. New vortex structures can be found at the outer side of the hummingbird for this time instance. The LEV, TV and SLEV1 are connected with each other to form a vortex loop near the outer wing tip region. Also, the LEV, SLEV2, TEV and RV are connected to form another vortex loop. It is worth noting that the directions of the vortex tube SLEV1 and SLEV2 are opposite. Similarly, the PVL can be observed and it is connected to the later newly formed vortex loop. At the inner side of the hummingbird, the LEV develops and the newly formed vortex loop grow larger. However, the major vortex structures stay the same as we described at $\mathrm{t} / \mathrm{T}=0.24$. 

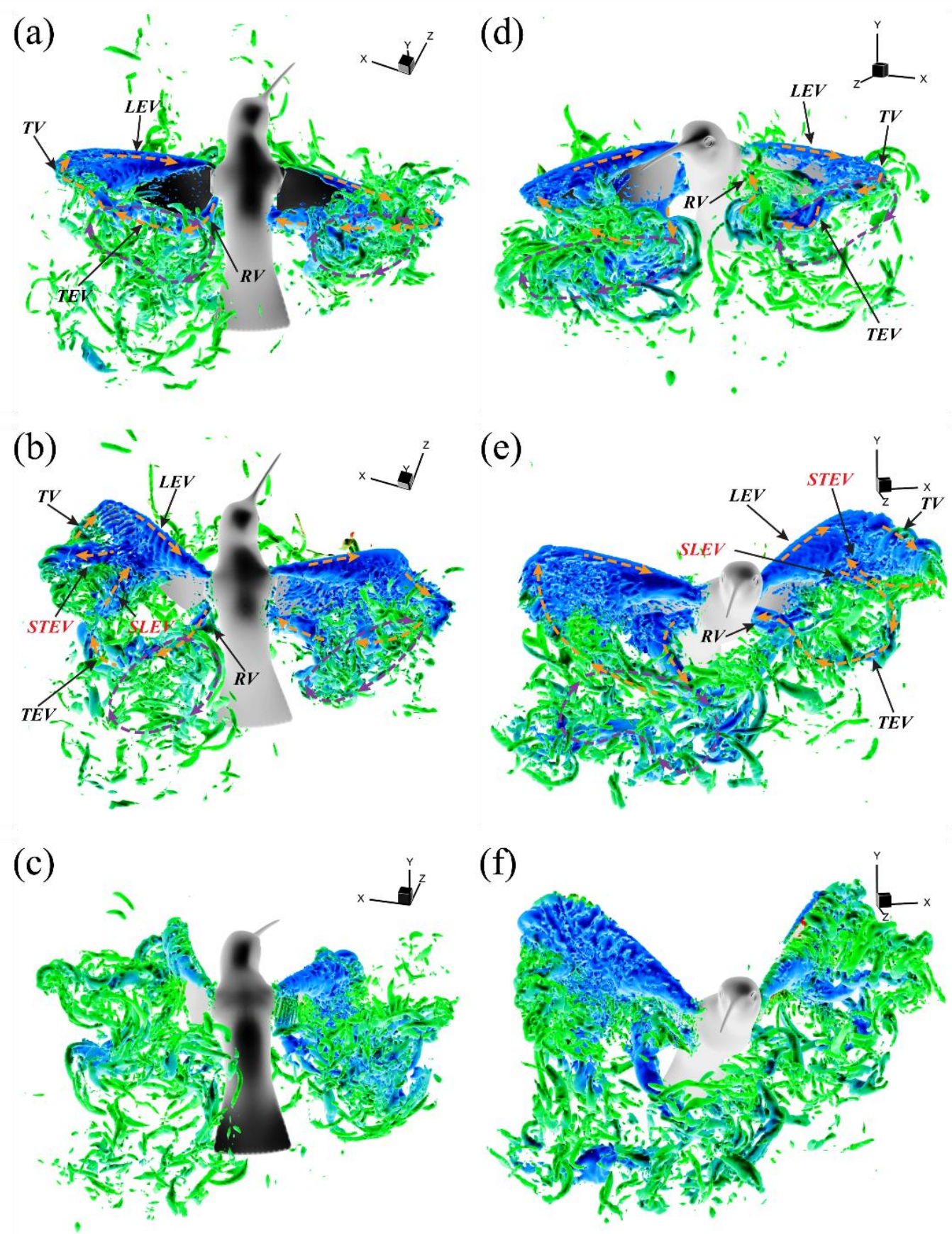

Figure 7-7. (a-f) Time course of vortex development of the 3rd stroke of the hummingbird pure yaw turn, visualized by the Q-criterion. The vortex structures are colored by nondimensional pressure. 
At the end of the downstroke ( $/ \mathrm{T}=0.48)$, as shown in Figure 7-7(c), the double vortex loop structure at the outer side of the hummingbird still can be observed. The vortices convect further downstream and the PVL starts to dissipate. The double vortex loop structure begins to merge to a single vortex loop.

During the upstroke (t/T=0.73), as shown in Figure 7-7(d), which corresponds to the peak of vertical force at upstroke (Figure 7-7a). The major vortex structures are similar to that at $\mathrm{t} / \mathrm{T}=0.24$, except for the more complex and stronger vortices due to larger angle of attack of the wings in upstroke (Figure 7-7c). At $\mathrm{t} / \mathrm{T}=0.88$, which corresponds to the peak of horizontal force at upstroke, the double loop vortex structure can be observed. Differently, it is the inner side of the hummingbird that presents the double loop vortex structure. At the end of upstroke, as shown in Figure 7-7(f), the double loop vortex structure still exists and the two vortex loops start to merge to a single vortex loop.

For all time instances discussed above, the inner side and outer side of the hummingbird show significant asymmetry in vortex wake structures. The unique double loop vortex structure exists in the outer side during downstroke and exists in the inner side during upstroke. Such double loop vortex structure is responsible for the wake asymmetry. A possible reason for the formation of the double loop structure is the involvement of the hummingbird body motion. At the downstroke, the outer wing sweeps longer distance due to the hummingbird body motion. In another hand, the inner wing sweeps longer distance during the upstroke due to the body motion. The kinematic difference of the inner and outer wings produced by the body motion may result in the unique double loop vortex structures, and further influence the wake structures of the inner and outer side of the hummingbird. 


\subsection{Near Wake Structure and Dual-Ring Wake Structure}

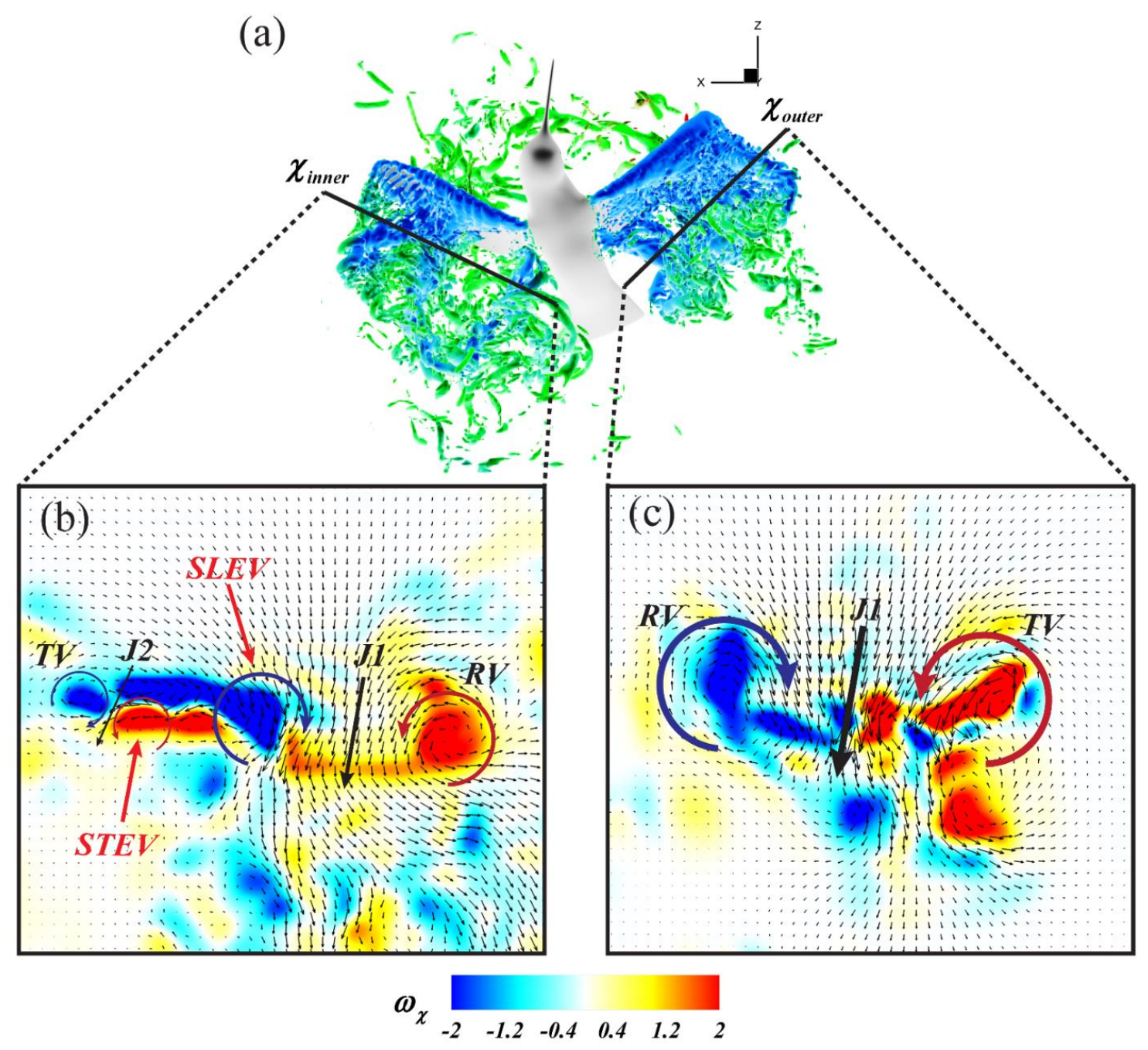

Figure 7-8. (a) Schematic plot showing slice locations; velocity vector field plots of (b) the inner side slice and (c) the outer side slice. (b) and (c) are colored by vorticity in the direction of the normal of the slice planes.

In order to better understand the wake topology of the hummingbird maneuvering flight, we cut the flow field with two 2D slices at the locations shown in Figure 7-8(a), and the 
corresponding time instance is $\mathrm{t} / \mathrm{T}=0.33$. As discussed before, at this time instance, the outer side of the hummingbird body shows double loop vortex structure, and the inner side of the hummingbird body presents normal vortex structures which is reported by previous researchers. A comparison of the two vortex structures can be conducted in this time instant. We can clearly see the SLEV and STEV from the slice of the outer wing shown in Figure 7-8(a). The first vortex loop is formed by connecting the LEV and TEV and SLEV near the wing tip region (near $70 \%$ wing span). The second vortex loop is formed by connecting the LEV, STEV and TEV near the mid wing region. The asymmetric wake topology between the inner and outer side of the hummingbird body is due to the existence of the dual-ring vortex structure in one side of the wing.

In order to better understand the wake topology and its effect to the aerodynamic performance, non-dimensional pressure is plotted to indicate the low pressure regions behind the wings of the hummingbird. Plotted in Figure 7-9(a) is one Iso-surface of the pressure corresponding to a non-dimensional pressure value of -1.0 at $\mathrm{t} / \mathrm{T}=0.33$. Noticeable in this figure is a large region of low pressure right behind the wings. However, for the outer side at $\mathrm{t} / \mathrm{T}=0.33$, the pressure Isosurface show branches near the mid wing region, which are due to the dual-ring vortex structure. Figure 7-9(b) shows the schematic of the correlation between identifiable vortex structures and regions of low pressure in Figure 7-9(a). The LEV, SLEV, STEV, TV, TEV and RV can be clearly identified in Figure 7-9(b) . The topology and formation of the dual-ring vortex structure are clearly revealed. 


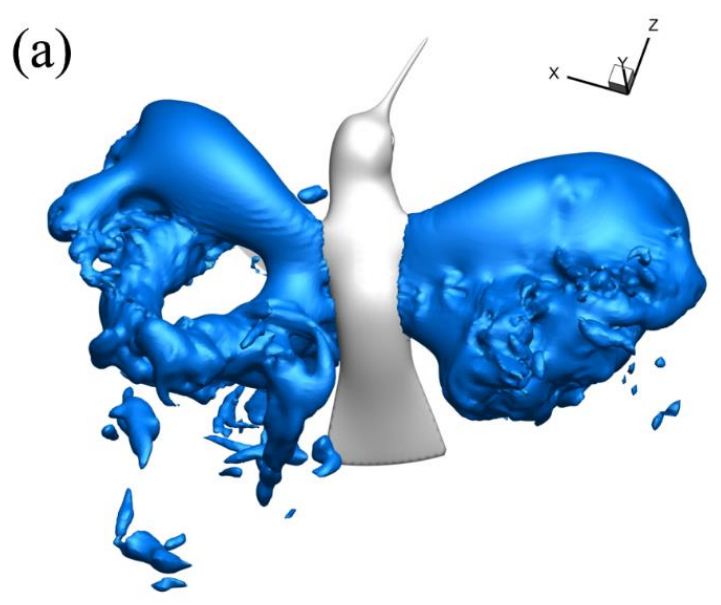

(b)

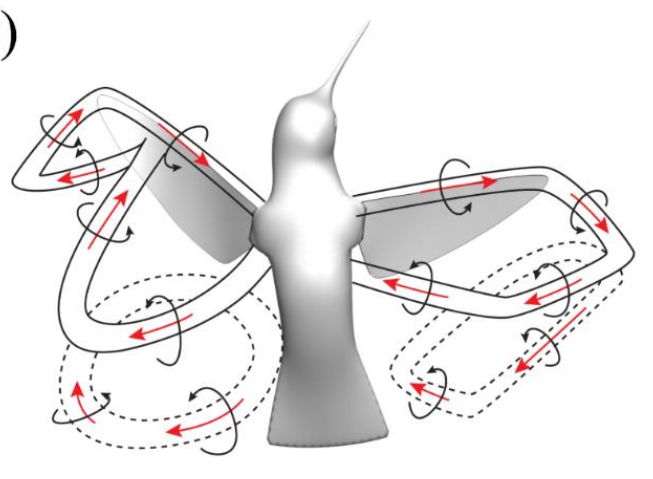

Figure 7-9. (a) Isosurface of pressure corresponding to a non-dimensional value of -1.0 at $\mathrm{t} / \mathrm{T}=0.37$ of the $3 \mathrm{rd}$ stroke of the hummingbird pure yaw turn; (b) wake schematic at $\mathrm{t} / \mathrm{T}=0.37$ of the $3 \mathrm{rd}$ stroke of the hummingbird pure yaw turn.

\subsection{Power Estimation}

In Figure 7-10, we plot both aerodynamic and inertial power using the same manner as we present the aerodynamic performances. In this plot, we show the power consumption of inner and outer wing separately to provide a better comparison of the asymmetry phenomenon. First, the instantaneous aerodynamic power is always positive over the entire cycle for both inner and outer wing, although its value is quite small near the wing reversal. The aerodynamic power plays a dominant role during the wing translation, and obtains the maximum near the mid-stroke. Unlike the aerodynamic power, the inertial power shows both positive and negative in the stroke cycle and more significant at wing reversal period. Specifically, the inertial power increase sharply owing to the wing acceleration, and then decrease until the mid-stroke, after that it starts to reverse the sign. In addition, the 
asymmetry between inner wing and outer wing is distinct for both aerodynamic and inertial power. For aerodynamic power, the peak value of inner wing is about 1.8 times higher than the outer wing. The magnitude of inertial power, on the other hand, only shows significant difference during the upstroke with around 2.1 times larger than the outer wing. Unlike the power consumption report in insect flight that the aerodynamic power play a dominate role over the course of the whole stroke, for hummingbird, both inertial and aerodynamic power are important.

(a)

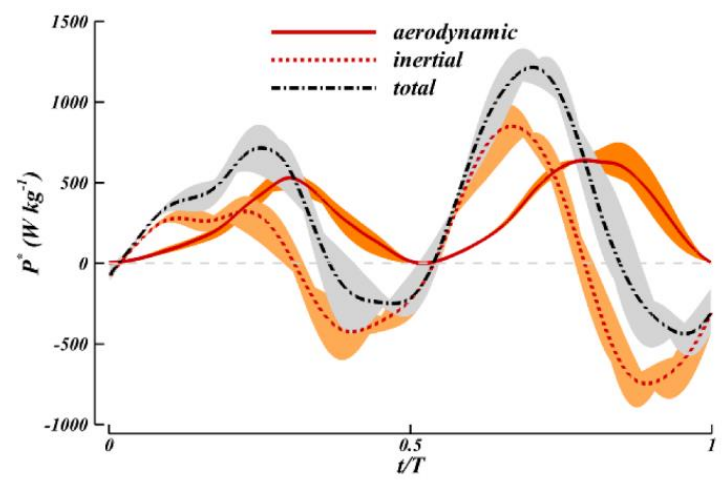

(b)

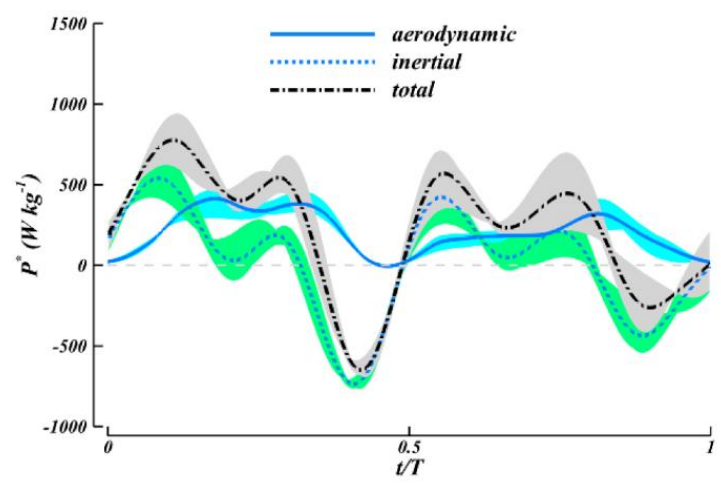

Figure 7-10. Average instantaneous specific power of in the turning phase of the hummingbird maneuvering flight. Both inner wing (a) and outer wing (b) are shown.

\subsection{Chapter Summaries}

A three-dimensional direct numerical simulation was performed for a maneuvering hummingbird with a very accurate wing and body motion reconstructed from high-speed images. The simulation captures the aerodynamic force and power characteristics in the entire maneuvering process and also details of the flow field, including the unsteady wake 
structures in both near and far field. Our results on the maneuvering hummingbird kinematics suggest that hummingbird sustained yaw turns by tiling up the outer wing stroke plane and suppressing the outer wing figure eight motion; also by increase the wing pitching angle in downstroke and decrease the wing pitching angle in upstroke to create wing angle of attack asymmetry between inner and outer wings. At the near wake, the hummingbird alters the inner wing pitching angle to control the LEV circulation, and therefore create asymmetry in drag forces of the two wings to generate yaw torque. The results show that in the turning phase, the LEV circulation of inner wing is about 2.5 times greater than that of the outer wing during downstroke and 2.1 times greater during the upstroke. Moreover, the lift force is produced near the wing tip region (90\% wing span) and the drag force is produce near the mid wing region (60\% wing span). At the far wake, strong asymmetric wake topology is identified. More importantly, a unique dual-ring vortex structure, which is the source of the wake asymmetry, is found in the wake of one of the two wings of the hummingbird. The dual-ring vortex structure corresponds to larger wing twisting and lower drag production, which creates unbalanced aerodynamic forces to help with the maneuver. 


\section{Concluding Remarks}

In the current dissertation, an integrated experimental and computational methodology has been developed to systematically study the flapping propulsion system in nature. The goal is to advance the fundamental knowledge of biological fluid dynamics in animal flight/swimming, and provide guidance for future optimal designs of bio-inspired flapping propulsors.

\subsection{Summary of Contributions}

In Chapter 2, the integrated methodology is introduced in detail. The corresponding major contributions of this work are described as follows:

(1) A highly versatile and accurate joint-based surface reconstruction method is developed to quantify the propulsor flexion and body kinematics of animals in free flight/swimming. The development of this method aims to improve the versatility and efficiency of the conventional reconstruction method, which is time-consuming and has several hard constraints regarding some details of the photogrammetry experiments, such as the camera location/orientation, the lens angle of view, marker points on the objects, etc.

(2) A spherical-coordinates-based singular value decomposition (SSVD) method is developed to perform low dimensional morphology analysis of flapping propulsors in nature. The conventional SVD method has several drawbacks regarding the distinctiveness and physics of the modes, such that large propulsor morphing can be found in several modes and some modes show great surface area change even though the surface 
area change in original motion is limited. The new method (SSVD) is developed to overcome the above issues.

(3) An immersed boundary method for deformable attaching bodies (IBM-DAB) is developed to handle direct numerical simulations (DNS) in extreme situations which are commonly exist in nature. Such as solid body with sharp edge and with deformable attaching membrane bodies.

(4) A highly efficient gradient-based parallel inexact curve searching optimizer is developed to explore design space of flapping propulsors. The computational cost of finding the searching direction and the step size is greatly reduced with the help of the new method.

In Chapter 3, the gradient-based optimization frame has been conducted to accomplish the optimal dynamic camber control of $2 \mathrm{D}$ flexible wing for maximizing the thrust production and propulsive efficiency. Direct Numerical Simulation (DNS) is used to gain unsteady flow around the flapping wing and its aerodynamic performance. Wing morphological parameters $\left(P_{R}, \beta_{m}, \varphi\right)$ are defined and two optimum sets (case Opt $C_{T}$ and case Opt $\eta$ ) of those parameters are found. Results show that the thrust production of the case Opt $C_{T}$ is increased by $29.1 \%$, and the propulsive efficiency of the case Opt $\eta$ is increased by $36.2 \%$ comparing to a case of a completely rigid flapping wing (case Rigid). The wake structures of these cases show different characteristics. Typical $2 \mathrm{~S}$ and $2 \mathrm{P}$ wake structures are identified in the case Opt $C_{T}$ and case Opt $\eta$, respectively. For the case Rigid, a $2 \mathrm{~S}$ wake structure with asymmetric property can be observed, which corresponds to 
obvious deflection of the mean flow. The flow modulation due to the dynamic camber formation could greatly improve the aerodynamic performance of the wing. The results of this work provide guidance for future optimal designs of dynamic cambered flapping propulsors.

In Chapter 4, computational optimization of dynamic twisting of pitching-rolling plates on aerodynamic performance is performed. First of all, we build a morphing plate model, which includes dynamic chordwise twisting in pitching-rolling motion inspired from biological propulsors. Thrust production, power consumption and flow structures of the model plates are obtained through an in-house immersed boundary method based CFD solver. Rigid pitching-rolling plates with different pitching amplitudes, varied from $15^{\circ}$ to $60^{\circ}$, are studied. The results show that the case with pitching amplitude of $30^{\circ}$ generates the highest thrust. Using this case as the baseline, optimal configurations of dynamic twisting for maximizing the thrust production and propulsive efficiency are found. The results show that the thrust production and propulsive efficiency of optimized plates can be improved by $6 \%$ and $43.1 \%$, respectively. Observations on the unsteady flow field of optimized cases show that the performance enhancements correspond to improved strength and attachment of leading-edge vortex. The findings of this work have indicated an alternative solution to designing future dynamic morphing propulsors applied to flying/swimming robots.

The next three chapters (Chapter 5, 6, and 7) discuss three applications which apply the integrated methodology to analyze flapping propulsion systems in nature, including a hovering dragonfly, a fast swimming orca, and a maneuvering hummingbird. The main purpose of choosing the three cases is to test validity of the integrated methodology in 
different situations. The three cases cover different flapping types, including pitchingrolling and pitching plunging; different propulsor properties, including membrane and solid body; and different motion complexities, including simple hovering/fast swimming and complex maneuvering. Another reason of choosing the three cases is that the current work is funded by different agencies, which have different focus areas.

In Chapter 5, the integrated methodology is used to study the complex morphing wing kinematics and the associated aerodynamics of a hovering dragonfly. SSVD analysis of the dragonfly's forewing motion reveals that the complicated wing motion can be represented by a low dimensional model contains two dominant SSVD modes, a flapping mode and a morphing mode. The low dimensional model contains $92 \%$ of the original motion, and can recover up to $96 \%$ of the aerodynamic performance. Parametric studies on the aerodynamic role of the dominant modes reveal that the morphing mode amplitude and phase are critical control parameters to achieve high aerodynamic performance. We further investigate optimal configurations of dominant modes on aerodynamic performance for the dragonfly wing. The corresponding optimized low dimensional wing models, which can beyond biological levels of aerodynamic performance, are obtained. The associated flow mechanisms are found to be the improved LEV attachment and the reduced TV strength. This is the first investigation of low dimensional wing morphological analysis as well as computational optimization on wing aerodynamic performance for a flying animal.

In Chapter 6, the integrated approach is used to study the morphing fluke kinematics and the associated hydrodynamics of a fast swimming orca. The SSVD analysis of the orca fluke motion shows that two dominant modes, a spanwise morphing mode and a chordwise morphing mode can be identified. The low dimensional model consist of these two modes 
(Mode 1+2) contains $74.3 \%$ of the original motions, and can fully recover the hydrodynamic performance. Parametric studies on the hydrodynamic role of the dominant modes reveal that the spanwise morphing mode is critical to achieve high thrust production while the chordwise morphing mode shows significant influence on propulsive efficiency. The associated flow mechanisms are found to be the improved LEV strength due to the spanwise morphing mode and the reduced secondary vortex strength due to the chordwise morphing mode. Further investigation on the far wake structures of the low dimensional mode (Mode 1+2) shows a unique tri-ring wake structure (upper ring: R1; middle ring: R2; lower ring: R3). The R2 is weakened and R3 is strengthened by removing the chordwise morphing mode, which degrades the propulsive efficiency. The R1 and R3 vanish in the far wake by removing the spanwise morphing, which decreases the thrust production. More importantly, this tri-ring vortex structure is closely related to the biology of cetaceans. Flow jet induced by R3 indicates additional vertical force production of cetaceans, which help them to go above the free surface to breathe, or to perform aerial jump. This is the first computational study on cetacean swimming. Also, this is the first report of the unique

\section{tri-ring wake structure generated by the orca fluke.}

In Chapter 7, the integrated methodology is applied to conduct direct numerical simulation for a hummingbird in a highly complex maneuvering flight. The simulation captures the aerodynamic force and power characteristics in the entire maneuvering process

and also details of the flow field, including the unsteady wake structures in both near and far field. Our results on the maneuvering hummingbird kinematics suggest that hummingbird sustained yaw turns by tiling up the outer wing stroke plane and suppressing the outer wing figure eight motion; also by increase the wing pitching angle in downstroke 
and decrease the wing pitching angle in upstroke to create wing angle of attack asymmetry between inner and outer wings. At the near wake, the hummingbird alters the inner wing pitching angle to control the LEV circulation, and therefore create asymmetry in drag forces of the two wings to generate yaw torque. The results show that in the turning phase, the LEV circulation of inner wing is about 2.5 times greater than that of the outer wing during downstroke and 2.1 times greater during the upstroke. Moreover, the lift force is produced near the wing tip region ( $90 \%$ wing span) and the drag force is produce near the mid wing region (60\% wing span). At the far wake, strong asymmetric wake topology is identified. More importantly, a unique duel-ring vortex structure, which is the source of the wake asymmetry, is found in the wake of one of the two wings of the hummingbird. The duel-ring vortex structure corresponds to larger wing twisting and lower drag production, which creates unbalanced aerodynamic forces to help with the maneuver. This is the first computational study on hummingbird maneuvering flight. Also, this is the first report of the unique dual-ring vortex structure generated by the flapping wings.

By comparing the results of the above three cases, we conclude that the morphing modes/kinematics of the biological flapping propulsors play important roles in their associated aero/hydrodynamic performance and wake structures. The dragonfly utilizes their wing morphing to reduce the wing tip vortex to improve the lift efficiency in hovering flight. The orca utilizes the fluke spanwise and chordwise morphing to form a tri-ring wake structure to improve the propulsive performance and produce additional vertical force for the purpose of breathing or aerial jumping. The hummingbird utilizes the wing twist to form a dual-ring wake structure in one of the two wings, which leads to the asymmetric 
wake developments of the inner and outer wings. The wake asymmetry results in drag asymmetry, which creates torque to sustain the yaw turn of the hummingbird.

\subsection{Future Work}

One possible extension of the current research includes exploring the design space of propulsor morphing kinematics for highly complex biological propulsion systems, such as maneuvering birds/insects/fishes, burst-and-coast fishes, etc. We have ongoing works on a sunfish which performs maneuvering motion to avoid obstacle, and a blue fish which performs burst-and-coast motion to save energy during the swimming. Such investigations could lead to valuable insights into enduring scientific mysteries about the flow mechanisms of high performance propulsion systems beyond biology to the extent of bioinspired flapping propulsion system design in the future.

Another possible extensive study is to perform proper orthogonal decomposition (POD) on the unsteady flow field of flapping propulsion system, and build a linkage between the POD modes of the flow field and the SSVD modes of the propulsor morphing kinematics. Once the linkage is built, the existing flow control mechanisms based on POD modes of the flow field will be much more straightforward to implement in engineered systems by altering the corresponding SSVD modes of the propulsor morphing kinematics. 


\section{Bibliography}

1. Jensen, M., Biology and physics of locust flight: lll. The aerodynamics of locust flight. Phil. Trans. R. Soc. Lond. B, 1956. 239: p. 511-552.

2. Weis-Fogh, O., [Investigations on the composition of tablets.]. Dan Tidsskr Farm, 1956. 30(Suppl 2): p. 276-88.

3. Dudley, R., The biomechanics of insect flight. 2000: Princeton University Press.

4. Birch, J.M. and M.H. Dickinson, Spanwise flow and the attachment of the leadingedge vortex on insect wings. Nature, 2001. 412(6848): p. 729-33.

5. Birch, J.M. and M.H. Dickinson, The influence of wing-wake interactions on the production of aerodynamic forces in flapping flight. Journal of Experimental Biology, 2003. 206(13): p. 2257-2272.

6. Birch, J.M., W.B. Dickson, and M.H. Dickinson, Force production and flow structure of the leading edge vortex on flapping wings at high and low Reynolds numbers. Journal of Experimental Biology, 2004. 207(7): p. 1063-1072.

7. Dickinson, M.H., F.O. Lehmann, and S.P. Sane, Wing rotation and the aerodynamic basis of insect flight. Science, 1999. 284(5422): p. 1954-1960.

8. Dickinson, M.H., Haltere-mediated equilibrium reflexes of the fruit fly, Drosophila melanogaster. Philos Trans R Soc Lond B Biol Sci, 1999. 354(1385): p. 903-16.

9. Dickinson, M.H. and K.G. Gotz, The wake dynamics and flight forces of the fruit fly Drosophila melanogaster. Journal of Experimental Biology, 1996. 199(9): p. 2085-2104.

10. Ellington, C.P., The Aerodynamics of Hovering Insect Flight .1. The Quasi-Steady Analysis. Philosophical Transactions of the Royal Society of London Series BBiological Sciences, 1984. 305(1122): p. 1-15.

11. Ellington, C.P., The Aerodynamics of Hovering Insect Flight .2. Morphological Parameters. Philosophical Transactions of the Royal Society of London Series BBiological Sciences, 1984. 305(1122): p. 17-40.

12. Ellington, C.P., The Aerodynamics of Hovering Insect Flight .3. Kinematics. Philosophical Transactions of the Royal Society of London Series B-Biological Sciences, 1984. 305(1122): p. 41-\&. 
13. Ellington, C.P., The Aerodynamics of Hovering Insect Flight .4. Aerodynamic Mechanisms. Philosophical Transactions of the Royal Society of London Series BBiological Sciences, 1984. 305(1122): p. 79-\&.

14. Ellington, C.P., The Aerodynamics of Hovering Insect Flight .5. A Vortex Theory. Philosophical Transactions of the Royal Society of London Series B-Biological Sciences, 1984. 305(1122): p. 115-144.

15. Ellington, C.P., The Aerodynamics of Hovering Insect Flight .6. Lift and Power Requirements. Philosophical Transactions of the Royal Society of London Series B-Biological Sciences, 1984. 305(1122): p. 145-181.

16. Ellington, C.P., Unsteady aerodynamics of insect flight. Symp Soc Exp Biol, 1995. 49: p. 109-29.

17. Ellington, C.P., et al., Leading-edge vortices in insect flight. Nature, 1996. 384(6610): p. 626-630.

18. Dickinson, W.J., Aldehyde oxidase in Drosophila melanogaster: a system for genetic studies on developmental regulation. Dev Biol, 1971. 26(1): p. 77-86.

19. Fry, S.N., R. Sayaman, and M.H. Dickinson, The aerodynamics of free-flight maneuvers in Drosophila. Science, 2003. 300(5618): p. 495-498.

20. Fry, S.N., R. Sayaman, and M.H. Dickinson, The aerodynamics of hovering flight in Drosophila. Journal of Experimental Biology, 2005. 208(12): p. 2303-2318.

21. Lehmann, F.O. and M.H. Dickinson, The changes in power requirements and muscle efficiency during elevated force production in the fruit fly Drosophila melanogaster. Journal of Experimental Biology, 1997. 200(7): p. 1133-1143.

22. Lehmann, F.O., The constraints of body size on aerodynamics and energetics in flying fruit flies: an integrative view. Zoology, 2002. 105(4): p. 287-295.

23. Lehmann, F.O., Aerial locomotion in flies and robots: kinematic control and aerodynamics of oscillating wings. Arthropod Structure \& Development, 2004. 33(3): p. 331-345.

24. Lehmann, F.O., The mechanisms of lift enhancement in insect flight. Naturwissenschaften, 2004. 91(3): p. 101-122. 
25. Lehmann, F.O., S.P. Sane, and M. Dickinson, The aerodynamic effects of wingwing interaction in flapping insect wings. Journal of Experimental Biology, 2005. 208(16): p. 3075-3092.

26. Lehmann, F.O. and S. Pick, The aerodynamic benefit of wing-wing interaction depends on stroke trajectory in flapping insect wings. Journal of Experimental Biology, 2007. 210(8): p. 1362-1377.

27. VandenBerg, C. and C.P. Ellington, The vortex wake of a 'hovering' model hawkmoth. Philosophical Transactions of the Royal Society of London Series BBiological Sciences, 1997. 352(1351): p. 317-328.

28. VandenBerg, C. and C.P. Ellington, The three-dimensional leading-edge vortex of a 'hovering' model hawkmoth. Philosophical Transactions of the Royal Society of London Series B-Biological Sciences, 1997. 352(1351): p. 329-340.

29. Wang, Z.J., Two dimensional mechanism for insect hovering. Physical Review Letters, 2000. 85(10): p. 2216-2219.

30. Wang, Z.J., The role of drag in insect hovering. J Exp Biol, 2004. 207(Pt 23): p. 4147-55.

31. Wang, Z.J., J.M. Birch, and M.H. Dickinson, Unsteady forces and flows in low Reynolds number hovering flight: two-dimensional computations vs robotic wing experiments. Journal of Experimental Biology, 2004 . 207(3): p. 449-460.

32. Wang, J.K. and M. Sun, A computational study of the aerodynamics and forewinghindwing interaction of a model dragonfly in forward flight. Journal of Experimental Biology, 2005. 208(19): p. 3785-3804.

33. Wang, Z.J., Dissecting insect flight. Annual Review of Fluid Mechanics, 2005. 37: p. $183-210$.

34. Weisfogh, T., Quick Estimates of Flight Fitness in Hovering Animals, Including Novel Mechanisms for Lift Production. Journal of Experimental Biology, 1973. 59(1): p. 169-230.

35. Lighthill, M.J., On the Weis-Fogh Mechanism of Lift Generation. J. Fluid Mech., 1973. 60: p. 1-17. 
36. Maxworthy, T., Experiments on the Weis-Fogh mechanism of lift generation by insects in hovering flight Part 1. Dynamics of the 'fling'. J. Fluid Mech., 1979. 93: p. 47-63.

37. Birch, Investigation of the near-field tip vortex behind an oscillating wing. Journal of Fluid Mechanics, 2005. 544: p. 201-241.

38. Maxworthy, T., The Fluid-Dynamics of Insect Flight. Annual Review of Fluid Mechanics, 1981. 13: p. 329-350.

39. van den Berg, C. and C.P. Ellington, The vortex wake of a 'hovering' model hawkmoth. Philosophical Transactions of the Royal Society of London. Series B: Biological Sciences, 1997. 352(1351): p. 317-328.

40. Sane, S.P. and M.H. Dickinson, The aerodynamic effects of wing rotation and a revised quasi-steady model of flapping flight. Journal of Experimental Biology, 2002. 205(8): p. 1087-1096.

41. Sun, M. and J. Tang, Unsteady aerodynamic force generation by a model fruit fly wing in flapping motion. J Exp Biol, 2002. 205(Pt 1): p. 55-70.

42. Ren, Y., et al. Aerodynamic role of dynamic wing morphing in hummingbird maneuvering flight. in APS Division of Fluid Dynamics Meeting Abstracts. 2014.

43. Shallcross, G., et al. Low Dimensional Analysis of Wing Surface Morphology in Hummingbird Free Flight. in APS Meeting Abstracts. 2015.

44. Swartz, S.M., et al., Mechanical properties of bat wing membrane skin. Journal of Zoology, 1996. 239: p. 357-378.

45. Ren, Y. and H. Dong. Low-dimensional Modeling and Aerodynamics of Flexible Wings in Flapping Flight. in 34th AIAA Applied Aerodynamics Conference. 2016.

46. Dong, H., et al., Computational modelling and analysis of the hydrodynamics of a highly deformable fish pectoral fin. Journal of Fluid Mechanics, 2010. 645: p. 345373.

47. Ren, Y., et al. 3D Kinematics and Hydrodynamic Analysis of Freely Swimming Cetacean. in APS Meeting Abstracts. 2015.

48. Haibo Dong, et al., An Integrated Analysis of a Dragonfly in Free Flight. 40th AIAA Fluid Dynamics Conference and Exhibit,2010-4390, 2010. 
49. Liu, Y.P. and M. Sun, Wing kinematics measurement and aerodynamics of hovering droneflies. Journal of Experimental Biology, 2008. 211(13): p. 2014-2025.

50. Walker, S.M., A.L.R. Thomas, and G.K. Taylor, Photogrammetric reconstruction of high-resolution surface topographies and deformable wing kinematics of tethered locusts and free-flying hoverflies. Journal of the Royal Society Interface, 2009. 6(33): p. 351-366.

51. Koehler, C., et al., 3D reconstruction and analysis of wing deformation in freeflying dragonflies. Journal of Experimental Biology, 2012. 215(17): p. 3018-3027.

52. Wootton, R.J., Leading edge section and asymmetric twisting in the wings of flying butterflies (Insecta, Papilionoidea). J. Exp. Biol, 1993.

53. Combes, S.a.D., TL, Shape, flapping and flexion: wing and fin design for forward flight. J. Exp. Biol. , 2001.

54. Combes, S.A.D., T. L., Flexural stiffness in insect wings. I. Scaling and influence of wing venation. J. Exp. Biol, 2003.

55. Combes, S.A.D., T. L., Flexural stiffness in insect wings. II. Spatial distribution and dynamic wing bending. J. Exp. Biol, 2003.

56. Sane, S., The aerodynamics of insect flight. . J. Exp. Biol. , 2003.

57. Lehmann, F., wings touch wakes: understanding locomotor force control by wakewing interference in insect wings. J. Exp. Biol. , 2008.

58. Valasek, J., Morphing aerospace vehicles and structures. Aerospace series. 2012, Chichester: Wiley. xx, 286 p.

59. Wang, H., et al., Measuring wing kinematics, flight trajectory and body attitude during forward flight and turning maneuvers in dragonflies. Journal of Experimental Biology, 2003. 206(4): p. 745-757.

60. Shyy, W., et al., Aerodynamics of Low Reynolds Number Flyers. 2011: Cambridge University Press.

61. Zhao, L., X.Y. Deng, and S.P. Sane, Modulation of leading edge vorticity and aerodynamic forces in flexible flapping wings. Bioinspiration \& Biomimetics, 2011. 6(3).

62. Sun, M. and H. Tang, Unsteady aerodynamic force generation by a model fruit fly wing in flapping motion. Journal of Experimental Biology, 2002. 205(1): p. 55-70. 
63. Dai, H., H. Luo, and J.F. Doyle, Dynamic pitching of an elastic rectangular wing in hovering motion. Journal of Fluid Mechanics, 2012. 693: p. 473-499.

64. Shoele, K. and Q. Zhu, Performance of a wing with nonuniform flexibility in hovering flight. Physics of Fluids, 2013. 25(4).

65. Toomey, J. and J.D. Eldredge, Numerical and experimental study of the fluid dynamics of a flapping wing with low order flexibility. Physics of Fluids, 2008. 20(7).

66. Eldredge, J.D., J. Toomey, and A. Medina, On the roles of chord-wise flexibility in a flapping wing with hovering kinematics. Journal of Fluid Mechanics, 2010. 659: p. $94-115$.

67. Vanella, M., et al., Influence of flexibility on the aerodynamic performance of a hovering wing. Journal of Experimental Biology, 2009. 212(1): p. 95-105.

68. Wan, H., H. Dong, and G.P. Huang, Hovering Hinge-Connected Flapping Plate with Passive Deflection. AIAA Journal, 2012. 50(9): p. 2020-2027.

69. Lee, T. and Y.Y. Su, Lift enhancement and flow structure of airfoil with joint trailing-edge flap and Gurney flap. Experiments in Fluids, 2011. 50(6): p. 16711684.

70. Ho, C.M. and Y.C. Tai, Review: MEMS and its applications for flow control. Journal of Fluids Engineering-Transactions of the Asme, 1996. 118(3): p. 437-447.

71. Brian C. Prock, T.A.W., and William A. Crossley, Morphing Airfoil Shape Change Optimization with Minimum Actuator Energy as an Objective, in 9th AIAA/ISSMO Symposium on Multidisciplinary Analysis and Optimization. 2002: Atlanta, Georgia.

72. Liu, Y., B. Cheng, and X. Deng, An experimental Study of Dynamic Trailing Edge Deflections on a Two Dimensional Translating Wing, in 31st AIAA Applied Aerodynamics Conference. 2013, AIAA.

73. Li, C., H. Dong, and Y. Ren, A Numerical Study of Flapping Plates Hinged with a Trailing-Edge Flap. AIAA Paper 2014-2049, 2014.

74. $\mathrm{Xu}, \mathrm{M}$, et al., Adjoint-based optimization of flapping plates hinged with a trailingedge flap. Theoretical and Applied Mechanics Letters, 2015. 5(1): p. 1-4. 
75. Young, J., et al., Details of Insect Wing Design and Deformation Enhance Aerodynamic Function and Flight Efficiency. Science, 2009. 325(5947): p. 15491552.

76. Bozkurttas, M., et al., Low-dimensional models and performance scaling of a highly deformable fish pectoral fin. Journal of Fluid Mechanics, 2009. 631: p. 311342.

77. Liang, Y.C., et al., Proper orthogonal decomposition and its applications - Part I: Theory. Journal of Sound and Vibration, 2002. 252(3): p. 527-544.

78. Barber, T.J., M.H. Ahmed, and N.A. Shafi. POD snapshot data reduction for periodic flows. in 43th Aerospace Sciences and Meeting Exhibit. 2005. Reno, HV.

79. Berkooz, G., P. Holmes, and J.L. Lumley, The Proper Orthogonal Decomposition in the Analysis of Turbulent Flows. Annual Review of Fluid Mechanics, 1993. 25 : p. 539-575.

80. Urtasun, R., et al., Mahalanobis motion generation. Tech Rep, 2004.

81. Tuncer, I.H. and M. Kaya, Optimization of flapping airfoils for maximum thrust and propulsive efficiency. AIAA journal, 2005. 43(11): p. 2329-2336.

82. Ren, Y. and H. Dong, Computational Optimization of Flexible Wing Aerodynamic Performance in Hover, in 30th AIAA Applied Aerodynamics Conference. 2012, American Institute of Aeronautics and Astronautics.

83. Alexa, M. and W. Muller, Representing animations by principal components. Computer Graphics Forum, 2000. 19(3): p. C411-C418.

84. Troje, N.F., Decomposing biological motion: A framework for analysis and synthesis of human gait patterns. Journal of Vision, 2002. 2(5): p. 371-387.

85. Song, J.L., H.X. Luo, and T.L. Hedrick, Three-dimensional flow and lift characteristics of a hovering ruby-throated hummingbird. Journal of the Royal Society Interface, 2014. 11(98).

86. Zheng, L.X., T.L. Hedrick, and R. Mittal, A multi-fidelity modelling approach for evaluation and optimization of wing stroke aerodynamics in flapping flight. Journal of Fluid Mechanics, 2013. 721: p. 118-154.

87. Wan, H., H. Dong, and K. Gai, Computational investigation of cicada aerodynamics in forward flight. J R Soc Interface, 2015. 12(102): p. 20141116. 
88. Liu, G., H.B. Dong, and C.Y. Li, Vortex dynamics and new lift enhancement mechanism of wing-body interaction in insect forward flight. Journal of Fluid Mechanics, 2016. 795: p. 634-651.

89. Pletcher, R.H., J.C. Tannehill, and D. Anderson, Computational fluid mechanics and heat transfer. 2012: CRC Press.

90. Bozkurttas, M., et al., Towards numerical simulation of flapping foils on fixed Cartesian grids. AIAA Paper, 2005. 81: p. 2005.

91. Schneider, G. and M. Zedan, A modified strongly implicit procedure for the numerical solution of field problems. Numerical Heat Transfer, 1981. 4(1): p. 1-19.

92. Ruge, J. and K. Stüben, Algebraic multigrid. Multigrid methods, 1987. 3(13): p. $73-130$.

93. Stuben, K., A review of algebraic multigrid. Journal of Computational and Applied Mathematics, 2001. 128(1-2): p. 281-309.

94. Mavriplis, D.J. and V. Venkatakrishnan, A 3D agglomeration multigrid solver for the Reynolds-averaged-Navier-Stokes equations on unstructured meshes. International Journal for Numerical Methods in Fluids, 1996. 23(6): p. 527-544.

95. Ye, T., et al., An accurate Cartesian grid method for viscous incompressible flows with complex immersed boundaries. Journal of Computational Physics, 1999. 156(2): p. 209-240.

96. Udaykumar, H.S., et al., A sharp interface cartesian grid method for simulating flows with complex moving boundaries. Journal of Computational Physics, 2001. 174(1): p. 345-380.

97. Mittal, R., et al., A versatile sharp interface immersed boundary method for incompressible flows with complex boundaries. Journal of Computational Physics, 2008. 227(10): p. 4825-4852.

98. Press, W.H. and Numerical Recipes Software (Firm), Numerical recipes in FORTRAN. 1992, Cambridge University Press,: Cambridge England ; New York, N.Y. p. 1 computer disk $31 / 2$ in.

99. Arora, J.S., Introduction to optimum design. 3rd ed. 2011, Boston, MA: Academic Press. xvi, 880 p. 
100. Li, C., H. Dong, and G. Liu, Effects of a dynamic trailing-edge flap on the aerodynamic performance and flow structures in hovering flight. Journal of Fluids and Structures, 2015. 58: p. 49-65.

101. Hedrick, T.L., B. Cheng, and X.Y. Deng, Wingbeat Time and the Scaling of Passive Rotational Damping in Flapping Flight. Science, 2009. 324(5924): p. 252-255.

102. Muijres, F.T., et al., Leading-edge vortex improves lift in slow-flying bats. Science, 2008. 319(5867): p. 1250-1253.

103. Combes, S.A. and T.L. Daniel, Shape, flapping and flexion: Wing and fin design for forward flight. Journal of Experimental Biology, 2001. 204(12): p. 2073-2085.

104. Dong, H., et al. An integrated analysis of a dragonfly in free flight. in 40th AIAA fluid dynamics conference and exhibit, AIAA2010-4390. 2010.

105. Lauder, G.V., et al., Locomotion with flexible propulsors: I. Experimental analysis of pectoral fin swimming in sunfish. Bioinspiration \& Biomimetics, 2006. 1(4): p. S25.

106. Wang, Z.J., Dissecting insect flight. Annu. Rev. Fluid Mech., 2005. 37: p. 183-210.

107. Walker, S.M., A.L. Thomas, and G.K. Taylor, Photogrammetric reconstruction of high-resolution surface topographies and deformable wing kinematics of tethered locusts and free-flying hoverflies. Journal of The Royal Society Interface, 2009. 6(33): p. 351-366.

108. Koehler, C., et al., 3D reconstruction and analysis of wing deformation in freeflying dragonflies. The Journal of experimental biology, 2012. 215(17): p. 30183027.

109. Lauder, G. and P. Madden, Fish locomotion: kinematics and hydrodynamics of flexible foil-like fins. Experiments in Fluids, 2007. 43(5): p. 641-653.

110. Young, J., et al., Details of insect wing design and deformation enhance aerodynamic function and flight efficiency. Science, 2009. 325(5947): p. 15491552.

111. Alben, S., Optimal flexibility of a flapping appendage at high Reynolds number. J. Fluid Mech, 2008. 614: p. 355-380. 
112. Liu, P. and N. Bose, Propulsive performance from oscillating propulsors with spanwise flexibility. Proceedings of the Royal Society of London. Series A: Mathematical, Physical and Engineering Sciences, 1997. 453(1963): p. 1763-1770.

113. H. DONG, M.B., R. MITTAL, and P.M.A.G.V. LAUDER, Computational modelling and analysis of the hydrodynamics of a highly deformable fish pectoral fin. J. Fluid Mech, 2010. 645.

114. Ren, Y. and H. Dong. Computational Optimization of Flexible Wing Aerodynamic Performance in Hover. in the 30th AIAA Applied Aerodynamics Conference. 2012.

115. Tangorra, J., et al., The application of conducting polymers to a biorobotic fin propulsor. Bioinspiration \& biomimetics, 2007. 2(2): p. S6.

116. Tangorra, J.L., et al., The effect of fin ray flexural rigidity on the propulsive forces generated by a biorobotic fish pectoral fin. The Journal of Experimental Biology, 2010. 213(23): p. 4043-4054.

117. Esposito, C.J., et al., A robotic fish caudal fin: effects of stiffness and motor program on locomotor performance. The Journal of experimental biology, 2012. 215(1): p. 56-67.

118. Dong, H., R. Mittal, and F.M. Najjar, Wake topology and hydrodynamic performance of low-aspect-ratio flapping foils. Journal of Fluid Mechanics, 2006. 566: p. 309-343.

119. Anderson, J.M., et al., Oscillating foils of high propulsive efficiency. Journal of Fluid Mechanics, 1998. 360: p. 41-72.

120. Read, D.A., F.S. Hover, and M.S. Triantafyllou, Forces on oscillating foils for propulsion and maneuvering. Journal of Fluids and Structures, 2003 17(1): p. 163183.

121. Techet, A.H., Propulsive performance of biologically inspired flapping foils at high Reynolds numbers. Journal of Experimental Biology, 2008. 211(2): p. 274-279.

122. Bandyopadhyay, P.R., et al., Relationship of roll and pitch oscillations in a fin flapping at transitional to high Reynolds number. Journal of Fluid Mechanics, 2012. 702: p. 298-331. 
123. Soria, J. and B.J. Cantwell, Identification and classification of topological structures in free shear flows, in Eddy Structure Identification in Free Turbulent Shear Flows, J.P. Bonnet and M.N. Glauser, Editors. 1993. p. 379-390.

124. Mittal, R., et al., Locomotion with flexible propulsors: II. Computational modeling of pectoral fin swimming in sunfish. Bioinspir Biomim, 2006. 1(4): p. S35-S41.

125. Warrick, D.R., B.W. Tobalske, and D.R. Powers, Aerodynamics of the hovering hummingbird. Nature, 2005. 435(7045): p. 1094-1097.

126. Warrick, D.R., B.W. Tobalske, and D.R. Powers, Lift production in the hovering hummingbird. Proceedings of the Royal Society B: Biological Sciences, 2009. 276(1674): p. 3747-3752.

127. Altshuler, D., et al., Wake patterns of the wings and tail of hovering hummingbirds. Experiments in Fluids, 2009. 46(5): p. 835-846.

128. Song, J., H. Luo, and T.L. Hedrick, Three-dimensional flow and lift characteristics of a hovering ruby-throated hummingbird. Journal of The Royal Society Interface, 2014. 11(98): p. 20140541.

129. Song, J., H. Luo, and T.L. Hedrick, Performance of a quasi-steady model for hovering hummingbirds. Theoretical and Applied Mechanics Letters, 2015.

130. Song, J., H. Luo, and T.L. Hedrick, Wing-pitching mechanism of hovering Rubythroated hummingbirds. Bioinspiration \& biomimetics, 2015. 10(1): p. 016007.

131. Tobalske, B.W., et al., Three-dimensional kinematics of hummingbird flight. Journal of Experimental Biology, 2007. 210(13): p. 2368-2382.

132. Sapir, N. and R. Dudley, Backward flight in hummingbirds employs unique kinematic adjustments and entails low metabolic cost. The Journal of experimental biology, 2012. 215(20): p. 3603-3611.

133. Altshuler, D.L., et al., Wingbeat kinematics and motor control of yaw turns in Anna's hummingbirds (Calypte anna). The Journal of experimental biology, 2012. 215(23): p. 4070-4084.

134. Tobalske, B.W., W.L. Peacock, and K.P. Dial, Kinematics of flap-bounding flight in the zebra finch over a wide range of speeds. Journal of Experimental Biology, 1999. 202(13): p. 1725-1739. 
135. Warrick, D.R. and K.P. Dial, Kinematic, aerodynamic and anatomical mechanisms in the slow, maneuvering flight of pigeons. Journal of Experimental Biology, 1998. 201(5): p. 655-672.

136. Hedrick, T.L., B.W. Tobalske, and A.A. Biewener, Estimates of circulation and gait change based on a three-dimensional kinematic analysis of flight in cockatiels (Nymphicus hollandicus) and ringed turtle-doves (Streptopelia risotia). Journal of Experimental Biology, 2002. 205(10): p. 1389-1409.

137. Catmull, E. and J. Clark, Recursively Generated B-Spline Surfaces on Arbitrary Topological Meshes. Computer-Aided Design, 1978. 10(6): p. 350-355.

138. Harbig, R.R., J. Sheridan, and M.C. Thompson, Reynolds number and aspect ratio effects on the leading-edge vortex for rotating insect wing planforms. Journal of Fluid Mechanics, 2013. 717: p. 166-192.

139. Mittal, R. and G. Iaccarino, Immersed boundary methods. Annual Review of Fluid Mechanics, 2005. 37: p. 239-261.

140. Seo, J.H. and R. Mittal, A Sharp-Interface Immersed Boundary Method with Improved Mass Conservation and Reduced Spurious Pressure Oscillations. J Comput Phys, 2011. 230(19): p. 7347-7363.

141. Vankan, J., A 2nd-Order Accurate Pressure-Correction Scheme for Viscous Incompressible-Flow. Siam Journal on Scientific and Statistical Computing, 1986. 7(3): p. 870-891.

142. Zang, Y., R.L. Street, and J.R. Koseff, A Non-Staggered Grid, Fractional Step Method for Time-Dependent Incompressible Navier-Stokes Equations in Curvilinear Coordinates. Journal of Computational Physics, 1994. 114(1): p. 1833.

143. Warrick, D.R., B.W. Tobalske, and D.R. Powers, Lift production in the hovering hummingbird. Proceedings of the Royal Society B-Biological Sciences, 2009. 276(1674): p. 3747-3752. 